\title{
Radionuclide Air Emissions Report for the Hanford Site Calendar Year 1995
}

B. P. Gleckler
L. P. Diediker

Westinghouse Hanford Company

S. J. Jette

K. Rhoads

M. J. Sula

Pacific Northwest National Laboratory

Date Published

June 1996

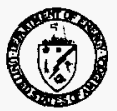

United States

Department of Energy

P.O. $80 \times 550$

Richland, Washington 99352

Approved for Public Release 
THS PACE INTENTIONALLY LEFT BLANK. 
TRADEMARK DISCLAINER

Reterence herein to any specific commercial procuct, process.

or service by trade name. trademark, manulaciufer. or

othe, wise, dues not necessarily constiluie or linpiy its

endorsemenl, recommendation, or lavoring by the Uniled

Stales Governmenl or any agency thereol of ils contractors or subconiraciors.

This report has been reproduced fion the best avai'able copy. Available in paper copy and microliche.

Available to the U.S. Department of Energy

and its coniractors from

Olfice of Scientilic and Technical Inlormation

PO Box 6 ?

Oak Ridgo. TN 37831

(615) 576.8401

Availabie to the public from the U.S. Departmenl of Ccmmerce

Nationa! Technical Information Service

5285 Porl Royal Road

Springlield. VA 22161

(703) 467.4650

Pointed in tha Uniled Siales of Anesice

DISCLM-5.CHP (8.91) 


\section{THIS PAGE INTENTIONALLY LEFT BLANK.}




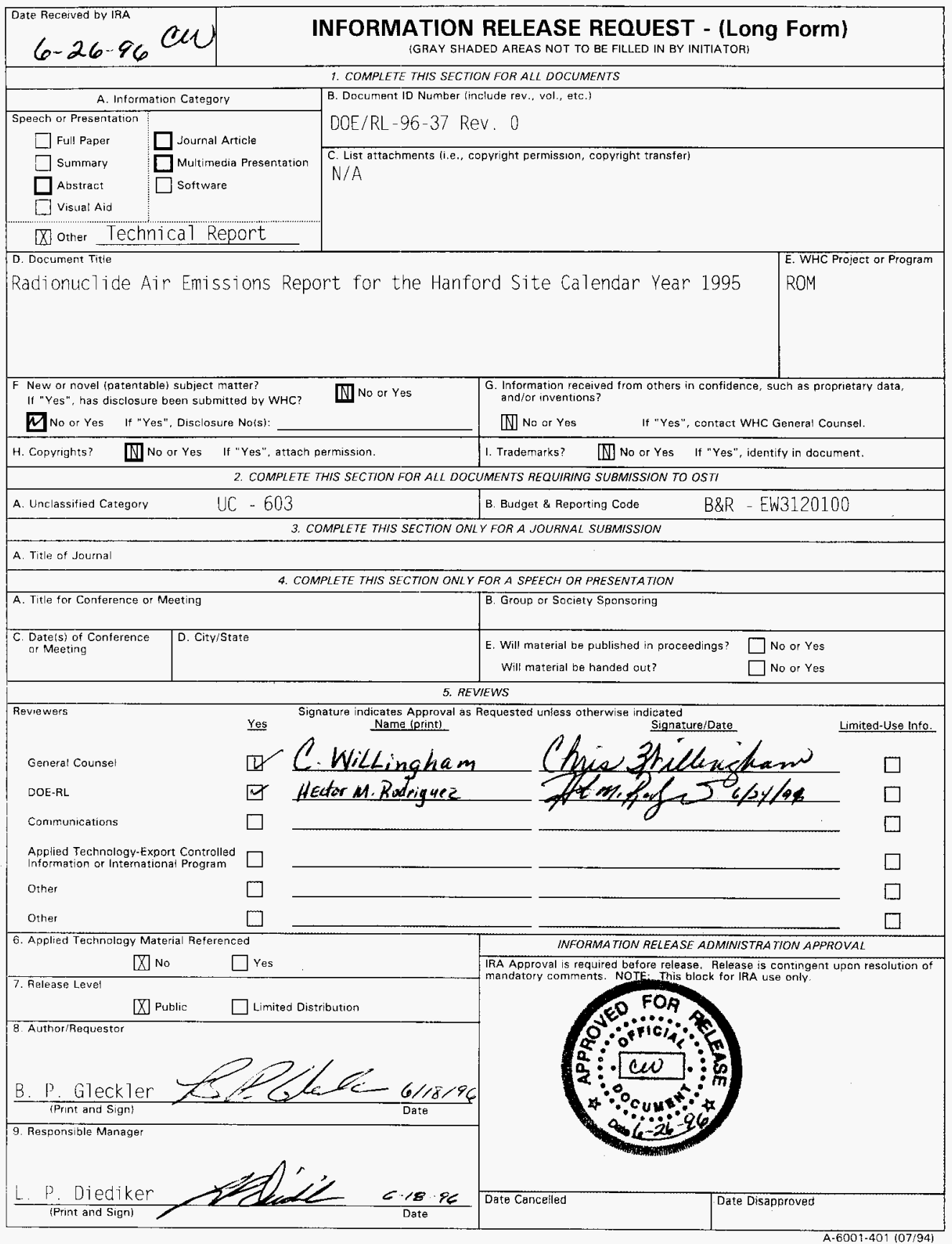


THIS PAGE INTENTIONALLY LEET BLANK. 
DOE/RL $-96-37$ ReV. 0 Document 10 Number

10. LEGENDS/NOTICES/MARKINGS (Required by WHC-CM-3-4 or Reviewer). Reviewer indicates applicable markings to be affixed or removed.

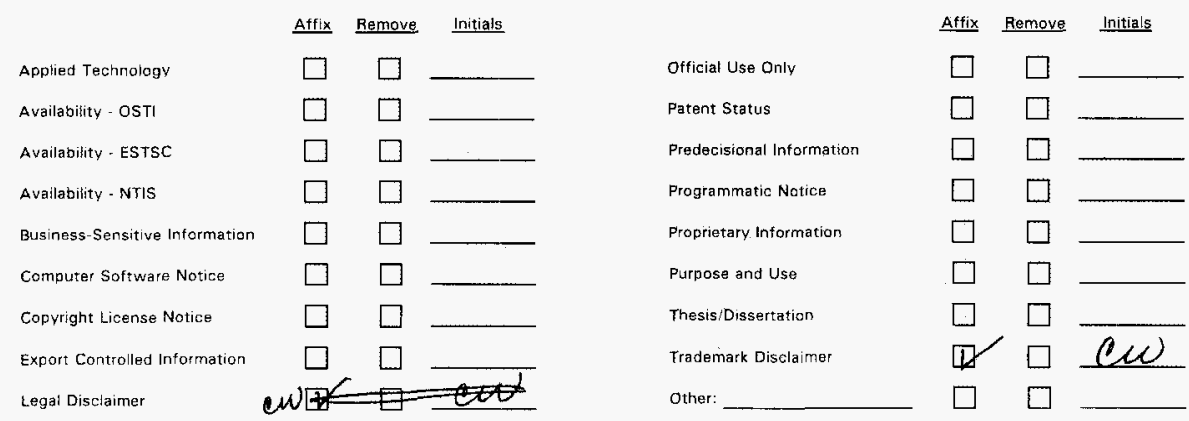

Limited Disclosure

11. MANDATORY COMMENTS (List only mandatory comments here. All other comments shall be made on the document and returned to the author.)

\begin{tabular}{|c|c|c|c|}
\hline $\begin{array}{c}\text { Reviewer } \\
\text { (Print \& Sign) }\end{array}$ & Date & $\begin{array}{l}\text { Resolved by Author/Requestor } \\
\text { (Print \& Sign) }\end{array}$ & Date \\
\hline & & & \\
\hline & & & \\
\hline & & & \\
\hline & & & \\
\hline & & 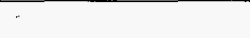 & \\
\hline & & & \\
\hline & & & \\
\hline & & & \\
\hline & & & \\
\hline & & & \\
\hline & & & \\
\hline & & & \\
\hline & & & \\
\hline & & & \\
\hline
\end{tabular}


THIS PAGE INTEMTIONALIY LEFT BLANK. 


\title{
RADIONUCLIDE AIR EMISSIONS REPORT FOR THE HANFORD SITE, CALENDAR YEAR 1995
}

\begin{abstract}
This repon documents radionuclide air emissions from the Hanford Site in 1995, and the resulting effective dose equivalent (EDE) to the maximally exposed member of the public, referred to as the "MEI." The report has been prepared and will be submitted in accordance with reporting requirements in the Code of Federal Regulations, Title 40, Protection of the Environment, Part 61, "National Emissions Standards for Hazardous Air Pollutants," Subpart H, "National Emission Standards for Emissions of Radionuclides Other than Radon from Department of Energy Facilities." This report has also been prepared for and will be submitted in accordance with the reporting requirements of the Washington Administrative Code Chapter 246-247, "Radiation Protection-Air Emissions."
\end{abstract}

The effective dose equivalent to the MEI from Hanford's 1995 point source emissions was $2.9 \times 10^{-3}$ mrem, which is well below the regulatory limit of $10 \mathrm{mrem} / \mathrm{yr}$. Radon and thoron emissions, exempted from 40 CFR 61 Subpart $H$, resulted in an effective dose equivalent to the MEI of $3.5 \times 10^{3} \mathrm{mrem}$. The effective dose equivalent to the $\mathrm{MEI}$ attributable to diffuse and fugitive emissions, was $1.8 \times 10^{-2} \mathrm{mrem}$. The total EDE from all Hanford 1995 air emissions was $2.4 \times 10^{-2}$ mrem. Note that the dose attributed to point source emissions is the only dose regulated by state and federal regulations.

Mr. Hector M. Rodriguez, of the U.S. Department of Energy, Richland Operations Office, may be contacted, at (509) 376-6421, about the information in this document. 
DOE/RL-96-37

This page intentionally left blank. 


\section{CONTENTS}

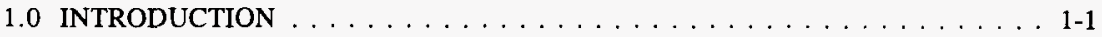

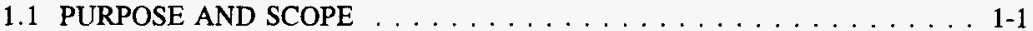

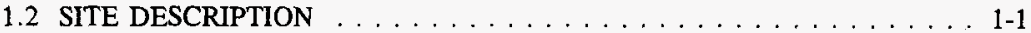

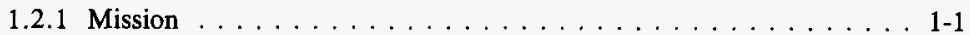

1.2 .2 Major Activities ..................... 1-2

1.2 .3 Site Characteristics . . . . . . . . . . . . . . . . 1-5

1.3 POINT SOURCE DESCRIPTIONS . . . . . . . . . . . . . . . 1-8

1.3.1 General Description and Reporting Criteria . . . . . . . . . . 1-8

1.3 .2100 Area Facilities . . . . . . . . . . . . . . . . . 1-11

1.3 .3200 East and West Area Facilities ............... . 1-12

1.3.4 300 Area Facilities . . . . . . . . . . . . . . . . . 1-22

1.3 .5400 Area Facilities $\ldots \ldots \ldots \ldots \ldots \ldots \ldots$ 1-29 . . . . . . . . . . . . . . . . . . . . . . . . . . . . . . . .

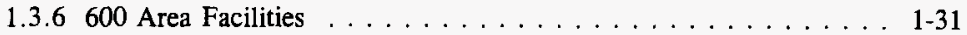

2.0 RADIONUCLIDE AIR EMISSIONS DATA ON POINT SOURCES $\ldots \ldots \ldots$. . . .

3.0 POINT SOURCE EMISSION DOSE ASSESSMENTS . . . . . . . . . . . 3-1

3.1 DESCRIPTION OF POINT SOURCE EMISSIONS DOSE MODEL . . . 3-1

3.2 SUMMARY OF INPUT PARAMETERS $\ldots \ldots \ldots \ldots \ldots \ldots$

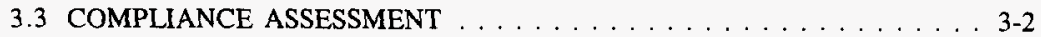

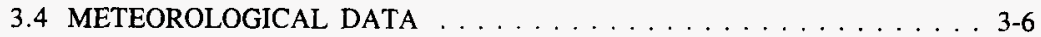

3.5 UNPLANNED RELEASES OF RADIONUCLIDES TO THE

ATMOSPHERE . . . . . . . . . . . . . . . . . . . . 3-6

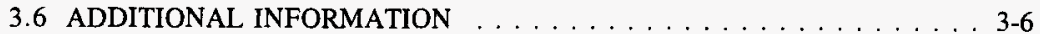

3.6.1 Projects Requiring Approval or Waiver Under 40 CFR $61 \ldots$. . . 3-6

3.6.2 Doses to the MEI due to Radon and Thoron Emissions . . . . . . . 3-6

3.6.3 Supplementary Data from Dose Modelling Results . . . . . . . . . . . 3-7

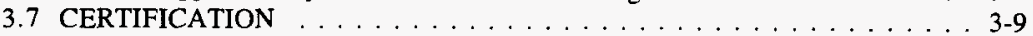

4.0 DIFFUSE AND FUGITIVE SOURCES OF EMISSIONS . . . . . . . . . . . 4-1

4.1 DIFFUSE AND FUGITIVE EMISSIONS MONITORING . . . . . . . . . . . . 4-1

4.1.1 Near-Facility Environmental Monitoring . . . . . . . . . . . . 4-2 . . . . . . . . .

4.1.2 Environmental Surveillance .................. . . 4-3

4.2 ESTIMATED DOSES FROM DIFFUSE AND FUGITIVE EMISSIONS . . 4-5

4.2.1 Description of Dose Assessment Method . . . . . . . . . . 4-5

4.2 .2 Results of Dose Assessment . . . . . . . . . . . . . . . . . 4-7

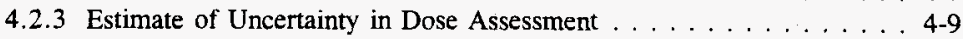

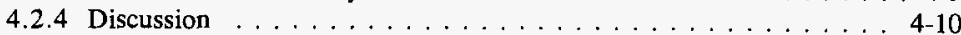

4.3 DIFFUSE AND FUGITIVE EMISSION SOURCES . . . . . . . . . . 4-11

4.3.1 Description of Diffuse and Fugitive Emission Sources . . . . . . . . 4-12

4.3.2 Description of Specific Diffuse and Fugitive Emission Sources . . . 4-15 
5.0 SUPPLEMENTAL INFORMATION $\ldots \ldots \ldots \ldots \ldots \ldots \ldots \ldots$ 5-1

5.1 POPULATION DOSE . . . . . . . . . . . . . . . . . 5-1

5.2 COMPLIANCE STATUS WITH SUBPARTS Q AND T OF 40 CFR $61 \ldots 5-1$

5.3 EMISSION OF RADON-220 FROM SOURCES CONTAINING

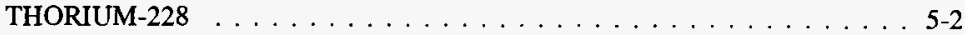

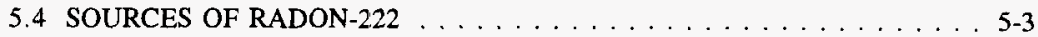

5.5 EMISSION POINTS SUBJECT TO MONITORING REQUIREMENTS OF 40 CFR 61.93(b) AND THE COSTS TO UPGRADE NONCOMPLIANT

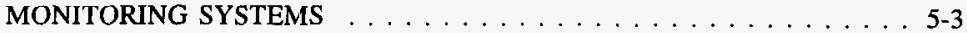

5.6 EMISSIONS ESTIMATES AND CONFIRMATORY MEASUREMENT DATA FOR SPECIFIC NOCs . . . . . . . . . . . . . . . 5-5

5.6.1 NOC for Portable Temporary Radioactive Airborne Emissions

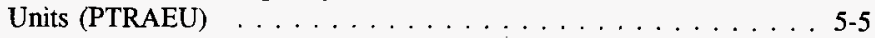

5.6.2 NOC for the Environmental Analytical Laboratory (EAL) $\ldots \ldots, 5-8$

5.6.3 Release Estimates for the L-070 Project . . . . . . . . . . . 5-8

5.7 STATUS OF COMPLIANCE WITH QA PROGRAM CRITERIA DESCRIBED IN APPENDIX B, METHOD 114,40 CFR $61 \ldots \ldots . .55$

6.0 REFERENCES . . . . . . . . . . . . . . . . . . 6-1

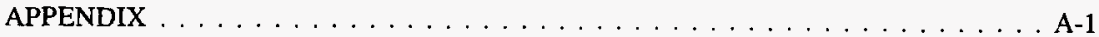




\section{LIST OF FIGURES}

1-1 Hanford Site Map . . . . . . . . . . . . . . . . . 1-3

1-2 100-N Area Point Sources . . . . . . . . . . . . . . . . . . . 1-9

1-3 100-K Area Emission Point Sources . . . . . . . . . . . . . . . . 1-10

1-4 200 East Area Emission Point Sources . . . . . . . . . . . . . . . . . 1-13

1-5 200 West Area Emission Point Sources . . . . . . . . . . . . . . . . . 1-14

1-6 300 Area Emission Point Sources . . . . . . . . . . . . . . . . . . . . . 1-23

1-7 400 Area Emission Point Sources . . . . . . . . . . . . . . . . . . 1-30

1-8 600 Area Emission Point Sources . . . . . . . . . . . . . . . . . . . 1-32

3-1 Historical Effective Dose Equivalents to the MEI . . . . . . . . . . . . . 3-3

4-1 Monthly Average Gross Beta Radioactivity in Airborne Particulate Samples, 1981

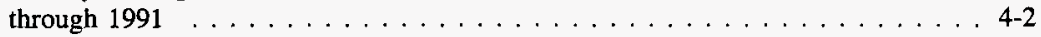

4-2 Near-Facility Ambient Air Sampling Locations . . . . . . . . . . . . 4-4

4-3 Environmental Surveillance Ambient Air Sampling Locations . . . . . . . . . . . 4 4-6

A-1 Meteorological Station Map and Wind Roses for Calendar Year $1995 \ldots \ldots$. . A-3

A-2 100 Area Wind Rose \& Histogram $\ldots \ldots \ldots \ldots \ldots \ldots \ldots \ldots$. . . . . . . . .

A-3 200 East Area Wind Rose \& Histogram $\ldots \ldots \ldots \ldots \ldots \ldots \ldots$ A-5 . . . . . .

A-4 200 West Area Wind Rose \& Histogram . . . . . . . . . . . . . A-6

A-5 300 Area Wind Rose \& Histogram . . . . . . . . . . . . . . . . . . A-7

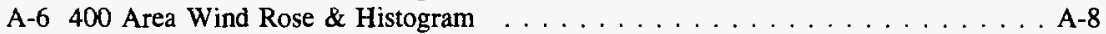




\section{LIST OF TABLES}

2-1 1995 Hanford Site Radionuclide Air Emissions Data For Major Point Sources . . . 2-3

2-2 1995 Hanford Site Radionuclide Air Emissions Data For Minor Point Sources . . 2-10

2-3 Distances and Directions from Area Release Locations to Nearest Receptors . . . 2-21

3-1 Release Estimates of Hanford Site Radionuclide Air Emissions in 1995 . . . . . . . . 3-4

3-2 1995 CAP88-PC Effective Dose Equivalent Estimates for the Maximally Exposed Individual (at Sagemore Road) Affected by Radionuclide Air Emissions from the Hanford Site . . . . . . . . . . . . . . . . . . . . . . . . . 3-5

3-3 1995 Effective Dose Equivalent to MEI (at Sagemore Road) from 300 Area Radon and Thoron Emissions . . . . . . . . . . . . . . . . . . . . 3-7

3-4 Total Doses from Individual Major Point Sources at the Hanford Site in 1995 . . 3-8

4-1 Estimated 1995 Hanford Site Diffuse and Fugitive Emissions and Resulting Effective Dose Equivalent . . . . . . . . . . . . . . . . . . . . . . . . 4-8

4-2 Types of Waste and Number of Waste Sites at the Hanford Site . . . . . . . . 4-13

4-3 Hanford Site Surface and Underground Contamination . . . . . . . . . . . 4-16

5-1 CY-1995 Emissions Estimates for PTREAUs ... . . . . . . . . . . . 5-5

5-2 CY-1995 Emissions Estimates for the Environmental Analytical Laboratory . . . . 5-8

5-3 CY-1995 Emissions Estimates for the L-070 Project . . . . . . . . . . . . . . . 5-8

A-1 Annual Average Dispersion Factor Around the 100 Area During 1995 for a 10Meter Release Height . . . . . . . . . . . . . . . . . . . . . . A-9

A-2 Annual Average Dispersion Factor Around the 200 Areas During 1995 for an 89Meter Release Height . . . . . . . . . . . . . . . . . A 10

A-3 Annual Average Dispersion Factor Around the 300 Area During 1995 for a 10Meter Release Height . . . . . . . . . . . . . . . . . . . . . . . A 11

A-4 Annual Average Dispersion Factor Around the 400 Area During 1995 for a 10-

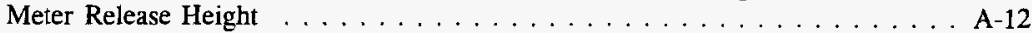

A-5 Radionuclide Data Used for CAP88-PC Dose Calculations at Hanford . . . . . . A-13

A-6 Exposure and Consumption Data for the Hanford Site . . . . . . . . . . . A-16

A-7 Hanford Site Meteorological Data - General Site Information . . . . . . . . . A-19 


\section{LIST OF TERMS}

\begin{tabular}{|c|c|}
\hline AMU & aqueous make-up \\
\hline BHI & Bechtel Hanford, Incorporated \\
\hline CAM & continuous air monitor \\
\hline CFR & Code of Federal Regulations \\
\hline CWC & Central Waste Complex \\
\hline $\mathrm{D} \& \mathrm{D}$ & decontamination and decommissioning \\
\hline DCRT & double-contained receiver tanks \\
\hline DOE & U.S. Department of Energy \\
\hline DST & double-shell tank \\
\hline $\mathrm{EDE}$ & effective dose equivalent \\
\hline EPA & U.S. Environmental Protection Agency \\
\hline ETF & Effluent Treatment Facility \\
\hline FFTF & Fast Flux Test Facility \\
\hline GTF & Grout Treatment Facility \\
\hline HEPA & high-efficiency particulate air (filter) \\
\hline HT & elemental tritium \\
\hline HTO & tritiated water vapor \\
\hline HVAC & heating, ventilation, and air conditioning \\
\hline MASF & Maintenance and Storage Facility \\
\hline MEI & maximally exposed individual (hypothetical) \\
\hline MW & mixed waste \\
\hline NESHAP & National Emission Standards for Hazardous Air Pollutants \\
\hline NOC & notice of construction \\
\hline PFP & Plutonium Finishing Plant \\
\hline PNL & Pacific Northwest Laboratory \\
\hline PNNL & Pacific Northwest National Laboratory (formerly PNL) \\
\hline PRF & Plutonium Reclamation Facility \\
\hline PTRAEU & Portable Temporary Air Emission Unit \\
\hline PUREX & Plutonium-Uranium Extraction (Plant) \\
\hline RCRA & Resource Conservation and Recovery Act \\
\hline REDOX & Reduction-Oxidation (Plant) \\
\hline RL & U.S. Department of Energy, Richland Operations Office \\
\hline RSB & Reactor Service Building \\
\hline SNM & special nuclear materials \\
\hline SST & single-shell tank \\
\hline Supply System & Washington Public Power Supply System \\
\hline TRIGA & Test Reactor and Isotope Production by General Atomics \\
\hline TRU & transuranic \\
\hline TRUSAF & Transuranic Waste Storage and Assay Facility \\
\hline $\mathrm{UO}_{3}$ Plant & Uranium-TriOxide Plant \\
\hline
\end{tabular}




\section{LIST OF TERMS (continued)}

WAC WDOH

WESF

WHC

WIPP

WSCF
Washington Administrative Code

Washington Department of Health

Waste Encapsulation Storage Facility

Westinghouse Hanford Company

Waste Isolation Pilot Plant

Waste Sampling and Characterization Facility 


\subsection{INTRODUCTION}

\subsection{PURPOSE AND SCOPE}

This report documents radionuclide air emissions from the Hanford Site in 1995, and the resulting effective dose equivalent to the maximally exposed member of the public, referred to as the "MEI." The report has been prepared and will be submitted in accordance with reporting requirements in the Code of Federal Regulations, Title 40, Protection of the Environment, Part 61, National Emissions Standards for Hazardous Air Pollutants, Subpart H, National Emission Standards for Emissions of Radionuclides Other than Radon from Department of Energy Facilities. This report has also been prepared for and will be submitted in accordance with the reporting requirements of the Washington Administrative Code Chapter 246-247, Radiation Protection-Air Emissions.

\subsection{SITE DESCRIPTION}

\subsubsection{Mission}

The federal government acquired the Hanford Site in 1943 to construct and operate facilities to produce plutonium for the atomic weapons program during World War II. For more than 40 years, Hanford Site facilities were dedicated primarily to producing plutonium for national defense and managing the wastes generated by chemical processing operations. In more recent years, defense programs have declined considerably while new programs have emerged involving research and development of waste disposal technologies, renewable energy technologies, and, in particular, cleanup of contamination from past operational practices.

The current Hanford Site mission includes the following activities:

- Environmental Restoration: Restoring approximately 1,500 inactive waste sites and either decontaminating or demolishing about 100 surplus facilities.

- Waste Management: Includes management of spent nuclear fuel, high level, low level, and transuranic radioactive wastes, other hazardous wastes, mixed radioactive and hazardous wastes, and sanitary wastes, as well as the remediation of high level radioactive wastes currently stored in underground tanks.

- Research and Development: Basic research in physical, biological, and environmental sciences, as well as applied research related to energy production, environmental restoration, and hazardous waste management. 
Activities related to defense production have ceased. N Reactor is currently being deactivated prior to being decommissioned. The Plutonium-Uranium Extraction (PUREX) Plant is in transition to be decommissioned. The Plutonium Finishing Plant (PFP), now set up to process plutonium scrap, did not operate in 1995. The 300 Area fuel fabrication facilities, where the uranium fuel elements for $\mathrm{N}$ Reactor were made, are deactivated and in transition to be decommissioned.

\subsubsection{Major Activities}

Six of the major U.S. Department of Energy (DOE) operating areas existing at the Hanford Site generated radioactive effluents in 1995. They were the 100, 200 East, 200 West, 300, 400, and 600 Areas (Figure 1-1). The 100 Areas include the $100 \mathrm{~K}$ Spent Fuel Storage Basins, and nine deactivated production reactors, with their support facilities, all located near the Columbia River. The 200 Areas reside on a plateau and are situated about $21.5 \mathrm{mi}(34.7 \mathrm{~km})$ northwest of the city of Richland, Washington and about $7.0 \mathrm{mi}$ $(11.3 \mathrm{~km})$ from the river. Facilities in the 200 East Area include the PUREX Plant, B Plant, and East Tank Farms. Facilities in the 200 West Area include the PFP, Uranium-TriOxide $\left(\mathrm{UO}_{3}\right)$ Plant, T Plant, 222-S Analytical Laboratory, waste management facilities, and Waste Sampling and Characterization Facility (WSCF) immediately outside the 200 West Area fence. The 300 Area, situated approximately $1.0 \mathrm{mi}(1.6 \mathrm{~km})$ due north of the city of Richland, contains research and development laboratories and the deactivated $\mathrm{N}$ Reactor fuel fabrication facilities. The 400 Area is the location of the Fast Flux Test Facility (FFTF), approximately $8.0 \mathrm{mi}(12.9 \mathrm{~km})$ north northwest of the city of Richland. The 600 Area includes all facilities not located in the other major operating areas.

Significant events in 1995, which were relevant to radioactive airborne emissions monitoring and reporting, are summarized below.

- The Effluent Treatment Facility (ETF) started operating in June of 1995. ETF started processing radioactive liquid effluents in November of 1995.

- Tank Farms operated the 242-A Evaporator in 1995, reducing the volume of some of Tank Farm's stored liquid radioactive waste.

- The 222-S Analytical Laboratory operated without interruption in 1995.

- The Waste Sampling and Characterization Facility (WSCF) operated without interruption in 1995 .

- The $\mathrm{UO}_{3}$ Plant had no emissions in 1995, since all its emission points were sealed off at the end of 1994. 
Figure 1-1. Hanford Site Map.

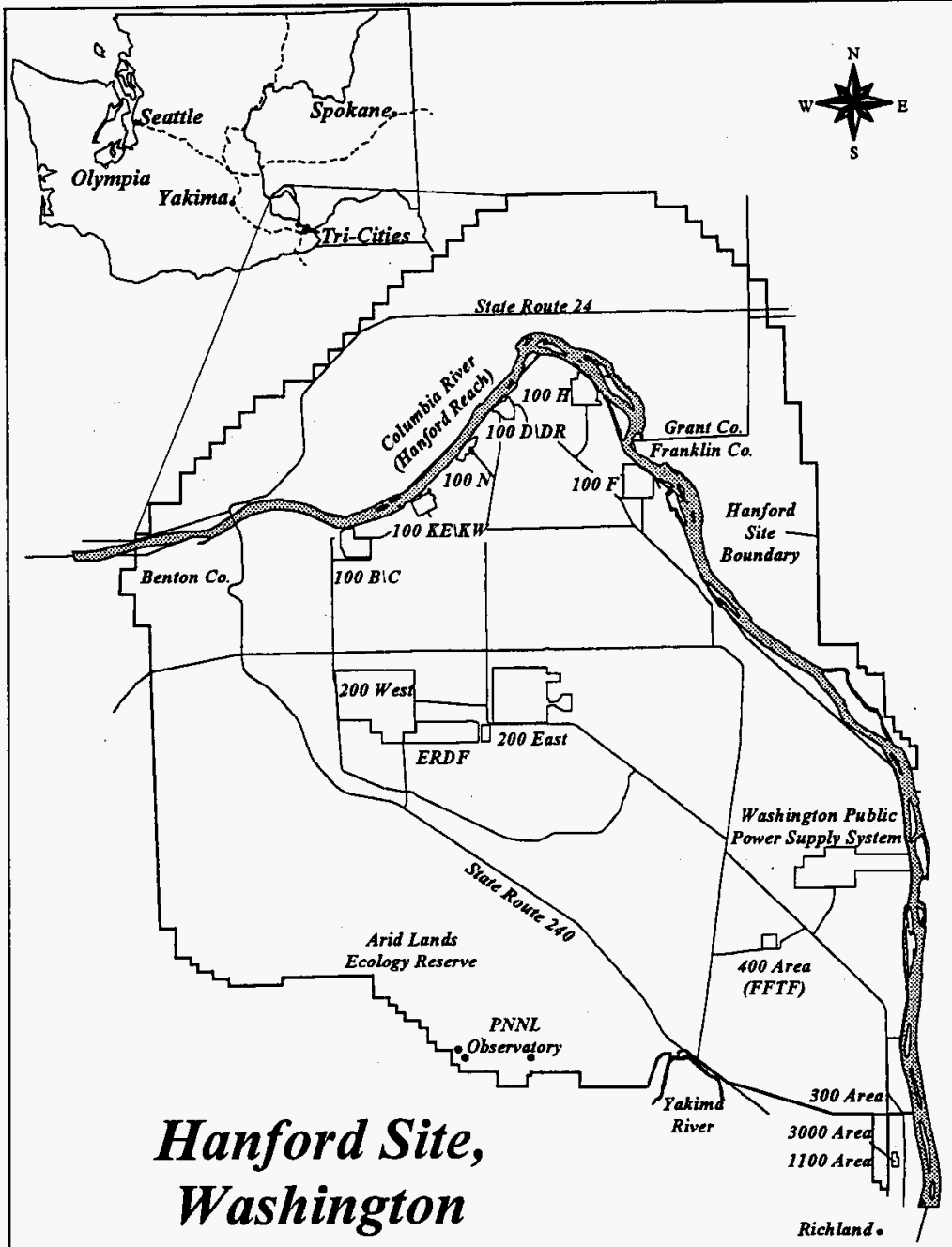
6.3
6.3
12.6 Kilometers 
- $\quad$ PFP continued to stabilize and store plutonium bearing materials in 1995.

Thermal stabilization activities were conducted on plutonium bearing solids and sludges, throughout 1995 . Laboratory development testing was conducted on plutonium solutions. Plutonium storage vault activates involved moving considerable quantities of special nuclear material to facilitate implementation of International Atomic Energy Agency (IAEA) safeguards on one of the storage vaults. Cleanout activities were also conducted in the $232-\mathrm{Z}$ Incinerator Building.

- The PUREX Plant did not operate in 1995. It has been in a deactivation mode since 1994.

- B Plant continued its revised primary mission of safely managing the radioactive inventories stored in its facilities.

- $\quad 100 \mathrm{~K}$ Spent Fuel Storage Basins continue to store irradiated fuel from N Reactor.

- The N Reactor has not operated since January 1987 and is currently being deactivated prior to being decommissioned. All nuclear fuel has been removed from the reactor core and the major piping systems have been drained.

- FFTF did not operate in 1995. In December 1993, DOE placed FFTF in transition to a radiologically and industrially safe shutdown condition. FFTF shutdown activities remained on schedule in 1995.

The DOE contractors and their management responsibilities for facilities at the Hanford Site that have radionuclide air emissions are identified below.

- Westinghouse Hanford Company (WHC). Manage the general operating contract which includes facilities such as: $N$ Reactor Fuel Fabrication Facilities, FFTF, Tank Farms, PUREX, B Plant, PFP, 222-S Analytical Laboratory, TRUSAF, WSCF, Central Waste Complex, Burial Grounds, the 200 Area Power Houses, and the $100 \mathrm{~K}$ Area; conduct liquid effluent monitoring, air emissions monitoring and near facility environmental surveillance at DOE facilities managed by WHC and BHI; provide Site support services, such as fire protection, central stores, the distribution of electrical power, and the generation of steam power.

- Battelle Memorial Institute. Manage the Pacific Northwest National Laboratory (PNNL) for DOE, including research and development in the physical, chemical, life, and environmental sciences; produce advanced methods of nuclear waste management; and conduct environmental monitoring on and off the Hanford Site and liquid effluent and air emission monitoring at the DOE facilities Battelle manages. 
- Bechtel Hanford, Incorporated (BHI). BHI is the Environmental Restoration Contractor which conducts surveillance and maintenance of deactivation and decommissioning activities at the following facilities: B Reactor, C Reactor, D and DR Reactors, F Reactor, H Reactor, N Reactor, REDOX (S Plant), $\mathrm{UO}_{3}$, and $\mathrm{U}$ Plant.

Privately and publicly owned facilities, capable of generating airborne radioactive emissions, are located at or near the Hanford Site. These facilities include: (1) a low-level radioactive-waste burial site operated by U.S. Ecology, Richland, Washington, on the 200 Area plateau; (2) the Washington Public Power Supply System (Supply System) power reactor (WNP-2) and office buildings, located near the Columbia River, several kilometers north of the 300 Area and east of the 400 Area; (3) the radio-analytical laboratory immediately south of the 300 Area operated by Quanterra, a subsidiary of IT Analytical Services; (4) the Siemens Nuclear Power Corporation fuel fabrication facility which is immediately adjacent to the southern boundary of the Hanford Site; (5) Interstate Nuclear Services, located one mile south of Hanford's southern boundary; and (6) Battelle's non-DOE research laboratories in the 3000 Area. Emissions from these privately owned facilities are not included in this report, because their emissions are monitored separately by state and federal agencies.

\subsubsection{Site Characteristics}

The Hanford Site (Figure 1-1) is located in a rural region of southeastern Washington State between $46^{\circ} 15^{\prime}$ and $46^{\circ} 45^{\prime}$ longitude and between $119^{\circ} 15^{\prime}$ and $120^{\circ} 00^{\prime}$ latitude, occupying an area of about $560 \mathrm{mi}^{2}\left(1,450 \mathrm{~km}^{2}\right)$. It lies about $200 \mathrm{mi}(320 \mathrm{~km})$ northeast of Portland, Oregon; $170 \mathrm{mi}(270 \mathrm{~km})$ southeast of Seattle, Washington; and $124 \mathrm{mi}(200 \mathrm{~km})$ southwest of Spokane, Washington.

Sections 1.1.3.1 through 1.1.3.4 provide summaries of the Hanford area's ecology, hydrology, climatology, and demography. More in-depth discussions on the Hanford Site's characteristics and activities is available in the Hanford Site National Environmental Policy Act (NEPA) Characterization (PNL 1995), the Climatological Summary for the Hanford Area (PNL 1993), the Vascular Plants of the Hanford Site (WHC 1992b), the Biological Diversity Inventory and Analysis at the Hanford Site: Insects (Zacks 1995), and the Status of Birds at the Hanford Site in Southeastern Washington (WHC 1992a).

1.2.3.1 Ecology. The Hanford Site is a relatively undisturbed area that has been botanically characterized as shrub-steppe, and contains numerous plant and animal species adapted to the region's semiarid environment. The Site consists of mostly undeveloped land with widely spaced clusters of industrial buildings located along the western shoreline of the Columbia River and at several locations in the interior of the Site.

Almost 600 species of plants have been identified on the Hanford Site. The dominant plants on the 200 Area Plateau are big sagebrush, rabbitbrush, spring hipsage, cheatgrass, 
and Sandberg's bluegrass, with cheatgrass providing half of the total plant cover. Cottonwood, willows, cattails and bulrushes grow along the banks of ponds and ditches. Near the 100 Areas, cheatgrass and riparian plants are the most prevalent plants. Big sagebrush, bitterbrush, rabbitbrush, cheatgrass, and Sandberg's bluegrass are common in the 300 and 400 Areas.

The main links in the food chain leading to man would be through mule deer, waterfowl, upland game birds, and fish. Other potential pathways leading to man through terrestrial food webs would be via elk and various types of wild plants, such as asparagus.

More than 400 species of terrestrial and aquatic insects have been found on the Hanford Site; however, the total number of species present is suspected to exceed 600 .

Twelve species of amphibians and reptiles are known to occur on the Hanford Site. The side-blotched lizard, gopher snake, yellow-bellied racer, and Pacific rattlesnake are the most abundant reptiles, while western and spade-footed toads along with Pacific tree and leopard frogs are the most abundant amphibians.

Over 240 species of birds have been identified on the Site, which include species such as: horned larks, western meadow larks, Canada geese, ringed-neck pheasants, California quail, mallard ducks, ring-billed gulls, California gulls, Forster's terns, blue herons, bald eagles, red-tailed hawks, cliff and bank swallows, chukar and gray partridges, morning doves, and more.

Approximately 39 species of mammals have been identified on the Hanford Site. The Columbia River shoreline supports populations of: beaver, muskrat, mink, raccoon, river otter, and striped skunk. Predators on the Site include: coyotes, mountain lion, bobcats, and badgers. Other mammals inhabiting terrestrial habitats include: Rocky Mountain elk, mule deer, black-tailed jackrabbits, cottontail rabbits, Townsend's ground squirrels, Great Basin pocket mice, deer mice, western harvest mice, northern grasshopper mice, montane voles, vagrant shrews, Merriam's shrew, and others.

1.2.3.2 Hydrology. The Columbia River is the second largest river in North America and the dominant surface-water body on the site. Originating in the mountains of eastern British Columbia, Canada, the Columbia River drains a total area of approximately $70,800 \mathrm{~km}^{2}$ $\left(27,300 \mathrm{mi}^{2}\right)$ en route to the Pacific Ocean. Flows through Hanford Reach fluctuate significantly and are controlled primarily by operations at the Priest Rapids Dam. Annual average flows at the vicinity of Priest Rapids over the last 68 years have averaged nearly $3360 \mathrm{~m}^{3}\left(122,000 \mathrm{ft}^{3}\right)$ per second. Daily average flows range from $1008 \mathrm{~m}^{3}$ to $7000 \mathrm{~m}^{3}$

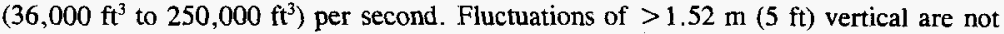
uncommon along the Hanford Reach. The width of the river varies from approximately $300 \mathrm{~m}(984 \mathrm{ft})$ to $1000 \mathrm{~m}(3,281 \mathrm{ft})$ within the Hanford Site.

The Yakima River, bordering a small length of the southern portion of the Hanford Site, has a low annual flow compared to the Columbia River. The average annual Flow, 
over the last 60 years, is about $104 \mathrm{~m}^{3}\left(3,712 \mathrm{ft}^{3}\right)$ per second, with an average monthly maximum of $490 \mathrm{~m}^{3}\left(17,500 \mathrm{ft}^{3}\right)$ per second. Approximately one third of the Hanford Site is drained by the Yakima River System.

Several springs such as Rattlesnake Springs, Snively Springs and Juniper Springs are located on the western part of the Site, some of which form small surface streams.

Rattlesnake Springs flow for about $3 \mathrm{~km}(1.6 \mathrm{mi})$ before disappearing into the ground. Cold Creek and its tributary, Dry Creek, are ephemeral streams draining to the Yakima River drainage system along the southern portion of the Hanford Site. Surface flow, when it occurs, infiltrates rapidly and disappears into the surface sediments in the western part of the Site. Other surface water on the Hanford Site consists of West Lake, numerous sloughs along the Columbia River, and a number of ditches and artificial ponds created for the disposal of waste water.

1.2.3.3 Climatology. The climate is dry and mild, and the area receives approximately $16 \mathrm{~cm}$ (6.3 in.) of precipitation annually. About $40 \%$ of the total precipitation occurs during November, December, and January; only $10 \%$ falls in July, August, and September. Approximately $45 \%$ of all precipitation from December through February is snow. The average minimum and maximum temperatures in July are $61{ }^{\circ} \mathrm{F}\left(16^{\circ} \mathrm{C}\right)$ and $91{ }^{\circ} \mathrm{F}\left(33^{\circ} \mathrm{C}\right)$, respectively. For January the average minimum and maximum temperatures are $21^{\circ} \mathrm{F}$ $\left(-6^{\circ} \mathrm{C}\right)$ and $38^{\circ} \mathrm{F}\left(3^{\circ} \mathrm{C}\right)$, respectively.

Monthly averaged wind speeds, taken from $50-\mathrm{ft}(15-\mathrm{m})$ above ground, range from about $9.1 \mathrm{mph}(15 \mathrm{~km} / \mathrm{h})$ in summer to $5.9 \mathrm{mph}(9.5 \mathrm{~km} / \mathrm{h})$ in winter. The prevailing winds are from the west-northwest and northwest, with occasional strong gusts. During the evening, the surface winds are influenced by cold-air drainage into the valleys. The region is a typical desert basin, in which frequent strong temperature inversions occur at night. These inversions generally deteriorate during the morning, leading to unstable atmospheric conditions in mid-morning and afternoon.

1.2.3.4 Demography. Estimates of population totals for Benton and Franklin Counties were 122,800 and 41,100 respectively, in 1993. Benton and Franklin counties have experienced a steady annual growth in population since 1987. Benton and Franklin Counties account for approximately $3 \%$ of the state's population. The 1993 estimates of populations in the TriCites are as follows: Richland 34,080, Kennewick 45,110, and Pasco 21,370. The combined population of Benton City, Prosser, and West Richland was estimated to be 11,000 in 1990 . The total population out to a distance of $80 \mathrm{~km}(50 \mathrm{mi})$ from the 200 Area meteorological tower was approximately 376,000 , in 1990 .

Land use in areas adjacent to the Hanford Site includes urban areas and industrial development, irrigated and dry-land farming, and grazing. Wheat normally represents the largest single crop in terms of area planted in Benton and Franklin counties. Corn, Alfalfa, hay, barley, and grapes are other major crops adjacent to the Site. Other significant crops include potatoes, apples, dry beans, asparagus, carrots, peaches, cherries, and pea seed. 
The area of the Hanford Site north of the Columbia River contains a state wildlife management area and a federal wildlife refuge. The northeast slope of the Rattlesnake Hills along the southwestern boundary of the Hanford Site is designated as the Fitzner-Eberhardt Arid Lands Ecology reserve, and is used by DOE for ecological research. The Hanford Site also is designated as a National Environmental Research Park.

\subsection{POINT SOURCE DESCRIPTIONS}

\subsubsection{General Description and Reporting Criteria}

Radionuclide air emissions from point sources generally are discharged from stacks and vents (further reference to stacks implies vents as well unless used as the proper name or description of a point source). Stack sizes, shapes, and discharge paths vary because of facility requirements at the time of construction. Discharge heights range from a few feet to $200 \mathrm{ft}(61 \mathrm{~m})$, and flow rates range from less than $100 \mathrm{ft}^{3} / \mathrm{min}(2,832 \mathrm{~L} / \mathrm{min})$ to more than $200,000 \mathrm{ft}^{3} / \mathrm{min}(5.7 \mathrm{million} \mathrm{L} / \mathrm{min})$. Stacks vary in design from horizontal to vertical, rectangular to cylindrical, and permanent to portable.

The following methods are used singly or in combination to remove radionuclides from most stack emissions: (1) high-efficiency particulate air (HEPA) filters; (2) sand filters; (3) deep-bed fiberglass filters; (4) fiberglass prefilters; (5) charcoal absorbers; and (6) water scrubbers. Generally, between one and three stages of HEPA filtration are used as the final particle removal method before an emission is exhausted to the atmosphere.

This report includes emissions estimates for 121 point sources on the Hanford Site. Point source emissions are reported in this document when the following criteria were met during 1995: (1) point source required continuous monitoring or periodic confirmatory measurements by 40 CFR 61 Subpart H or WAC 246-247, (2) point source was registered with Washington State Department of Health (WDOH), (3) the point source normally had radionuclide emissions or potentially had radionuclide emissions. Point sources not included in this report did not meet the previous criteria or were rendered inoperable (e.g. sealed off) before 1995 , as indicated by the previous year's annual report.

In 1995, 94 WHC and 35 PNNL point sources were reassessed (WHC 1995b, PNNL 1995). The assessments re-evaluated each point source's unmitigated potential to emit, to determine the applicable monitoring and reporting requirements under 40 CFR 61 Subpart $H$ regulations. The results from the reassessment change the regulatory status of several point sources from the status indicated by the 1994 assessments (WHC 1994a and 1994b). 
Figure 1-2. 100-N Area Point Sources.

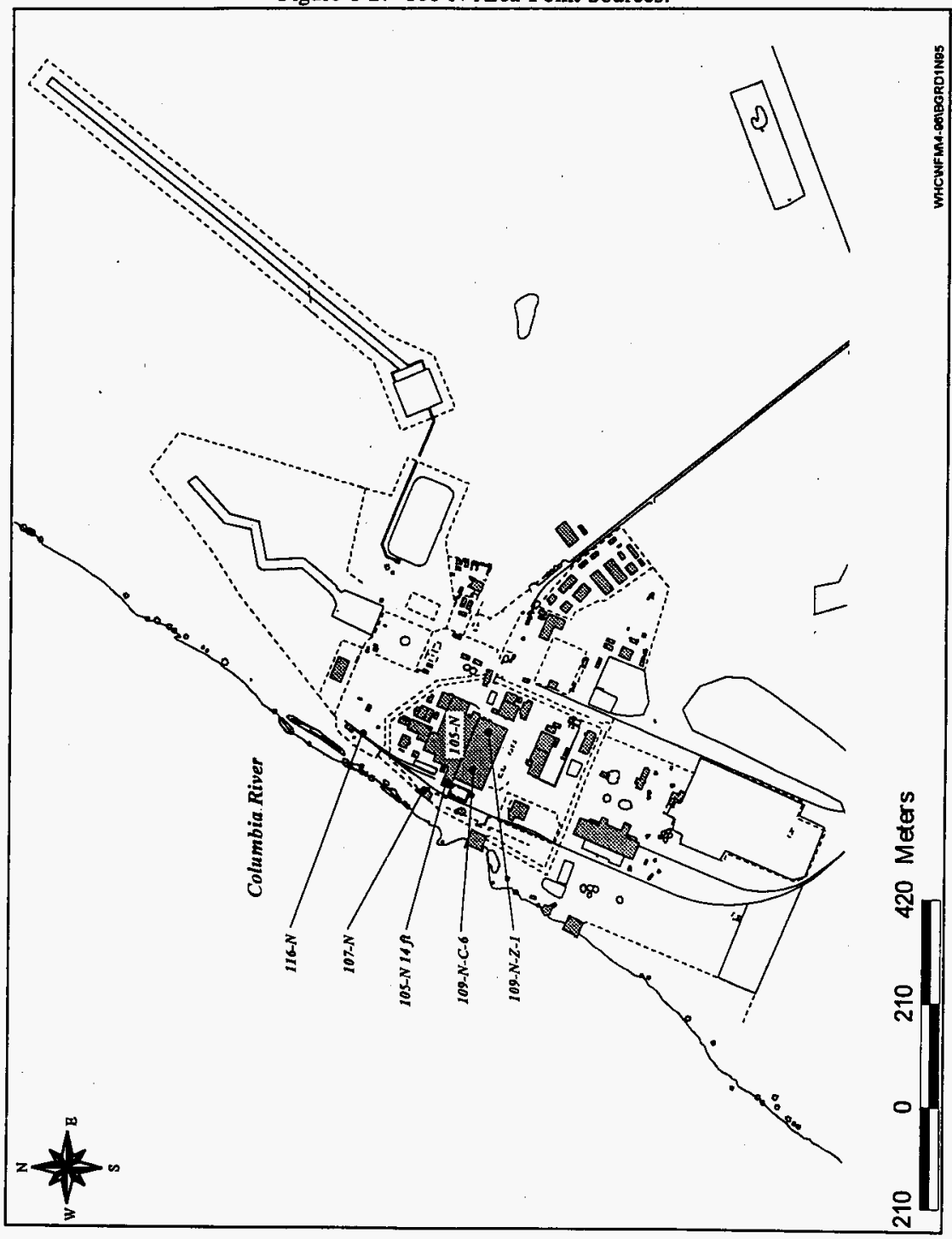


DOE/RL-96-37

Figure 1-3. 100-K Area Emission Point Sources.

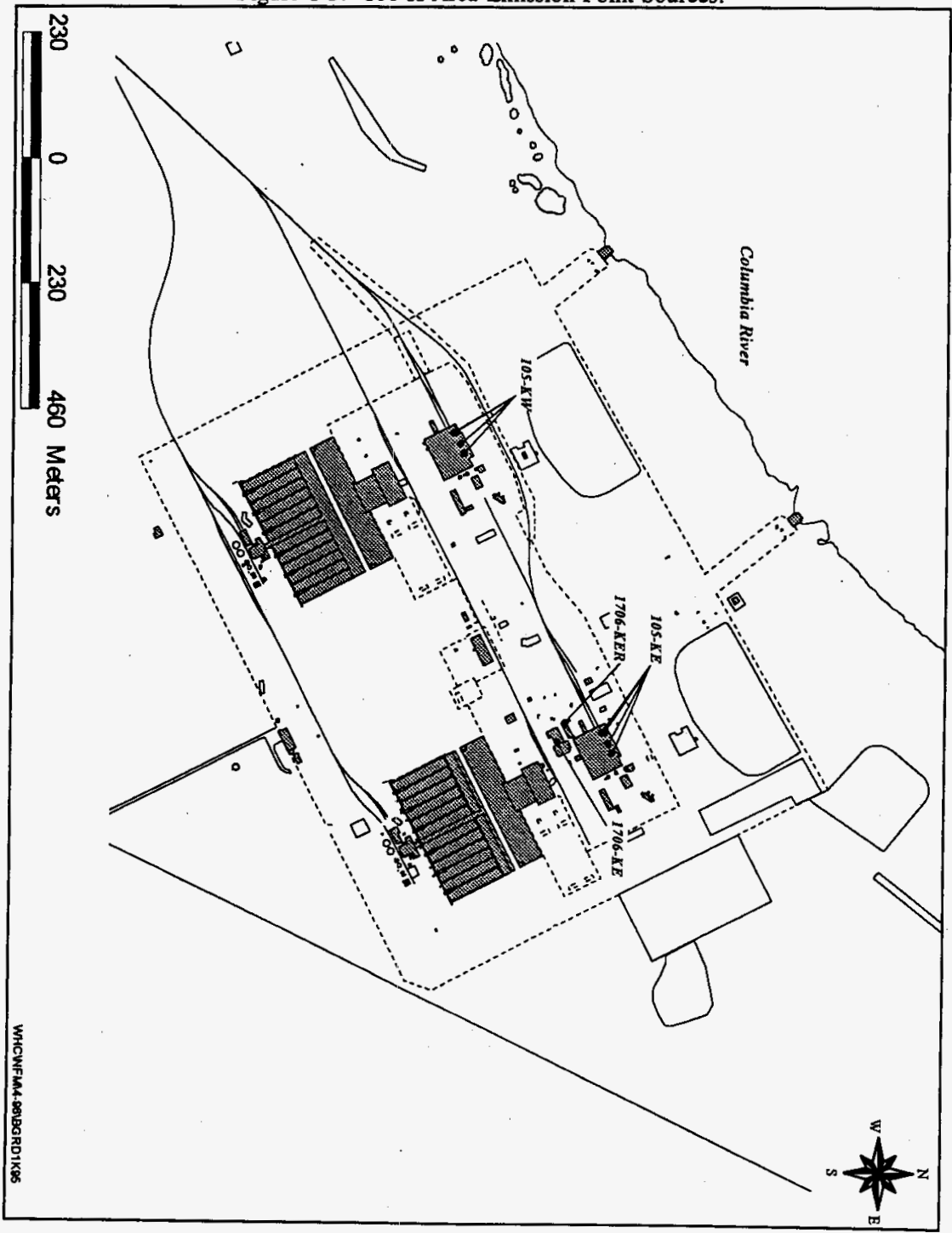




\subsubsection{Areas Facilities}

The 100 Areas contain eight inactive production reactors, the $\mathrm{N}$ Reactor (now in transition to deactivation and decommissioning), and associated support facilities. Radionuclide air emission points at facilities in the 100-N Area and 100-K Area are described briefly below and shown respectively in Figures 1-2 and 1-3.

1.3.2.1 N Reactor. This dual-purpose reactor operated until 1987, producing electrical power for the regional energy system and plutonium for weapons production. The reactor and associated facilities are currently being deactivated prior to decommissioning.

- 116-N. This stack exhausts filtered air from the 105-N Reactor Building. Emission monitoring consists of a record sampler. In $1993 \mathrm{~N}$ Reactor ventilation was reconfigured such that the $105-\mathrm{N}$ Basin Transfer Area emissions exhaust through the 116-N stack.

- 109-N-Z-I. When this vent is operational, unfiltered air was released through it from 109-N Steam Generator Cells 1 through 5. Emission monitoring consisted of a record sampler and a gross-gamma-energy monitor. This vent did not operate in 1995 and was deregistered in 1995.

- 109-N-C-6. When this vent is operational, unfiltered air is released through it from Steam Generator Cell 6 in the 109-N Building. Emission monitoring consisted of a record sampler and a gross-gamma-energy monitor. This vent did not operate in 1995 and was deregistered in 1995.

- 107-N. This vent exhausts filtered air from the 107-N Basin Recirculation Building. Emissions monitoring consists of a record sampler.

- 105-N 14-Ft. Decontamination Room. This stack exhausts filtered air from a small room in which radioactively contaminated tools are decontaminated. Emissions are continuously monitored by a record sampler.

1.3.2.2 100-K East and West Areas. These areas contain two retired reactors awaiting decommissioning, two water-filled storage basins storing irradiated nuclear fuel, and radiological analysis laboratories.

- 105-KE. This point source consists of four powered vents exhausting unfiltered air from the spent fuel storage basin in the 105-KE Building. Emission monitoring consists of three record samplers.

- 105-KW. This point source consists of four powered vents exhausting unfiltered air from the spent fuel storage basin in the 105-KW Building. Emission monitoring consists of three record samplers. 
- 1706-KE. This stack exhausts filtered air from the 1706-KE Laboratory. Emission monitoring consists of a record sampler.

- 1706-KER. This stack exhausts filtered air from the basement area (approximately $8.2 \mathrm{~m}$ [27 feet] below grade) of the 1706-KE Laboratory. Emission monitoring consists of a record sampler.

\subsubsection{East and West Area Facilities}

The 200 Areas contain facilities for chemical separations, processing, and waste handling and disposal. The radionuclide air emission discharge points in the 200 East Area are shown in Figure 1-4 and those in the 200 West Area, in Figure 1-5. The PUREX Plant and related tank farm and evaporator facilities discharge volatile forms of radionuclides, specifically ${ }^{3} \mathrm{H},{ }^{106} \mathrm{Ru},{ }^{125} \mathrm{Sb}$, and ${ }^{129} \mathrm{I}$. When operating, the PUREX Plant discharged ${ }^{85} \mathrm{Kr}$, but it has not operated fully since December 1989. No activities conducted in the 200 Areas in 1995 resulted in a discharge of ${ }^{85} \mathrm{Kr}$.

1.3.3.1 PUREX Plant. The PUREX Plant is a nuclear-fuel reprocessing plant constructed in 1956 and operated until 1972, when it was placed on standby status until November of 1983. In November of 1983 the PUREX Plant resumed operations, processing a backlog of irradiated fuel from $\mathrm{N}$ Reactor. The plant was again placed in standby following a stabilization run that ended in March 1990. Shutdown orders were issued to PUREX in December 1992, and as a result, the plant is being deactivated.

- 291-A-1. This stack exhausts filtered air from canyon ventilation (Cells A to $\mathrm{M})$, and vessel and condenser vents. Emission monitoring consists of a record sampler, a silver zeolite cartridge, an alpha continuous air monitor (CAM), and a beta-gamma CAM.

- 296-A-1. This stack exhausts filtered air from $N$ and Q Cells, Product Removal rooms, and gloveboxes in these rooms. Emission monitoring consists of a record sampler and an alpha CAM.

- 296-A-2. This stack exhausts filtered air from the west sample gallery hoods. Emission monitoring consists of a record sampler.

- 296-A-3. This stack exhausts filtered air from hoods in the east sample gallery. Emission monitoring consists of a record sampler.

- 296-A-5A and 296-A-5B. These stacks exhaust filtered air from laboratory hoods. Emission monitoring for each stack consists of a record sampler.

- 296-A-6. This stack exhausts filtered room air from the east sample gallery and $\mathrm{U}$ Cell. Emission monitoring consists of a record sampler. 


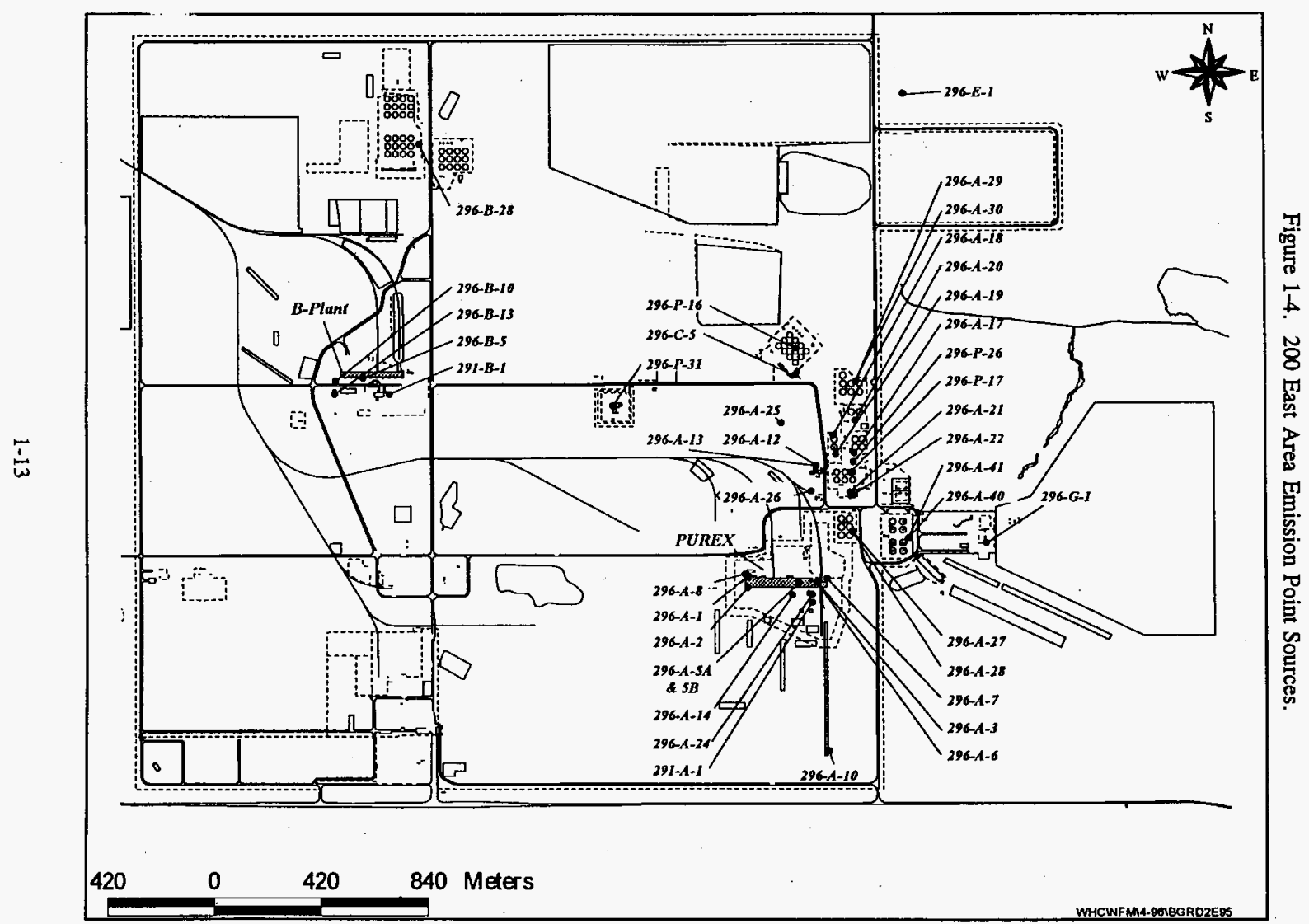

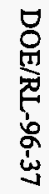


DOE/RL-96-37

Figure 1-5. 200 West Area Emission Point Sources.

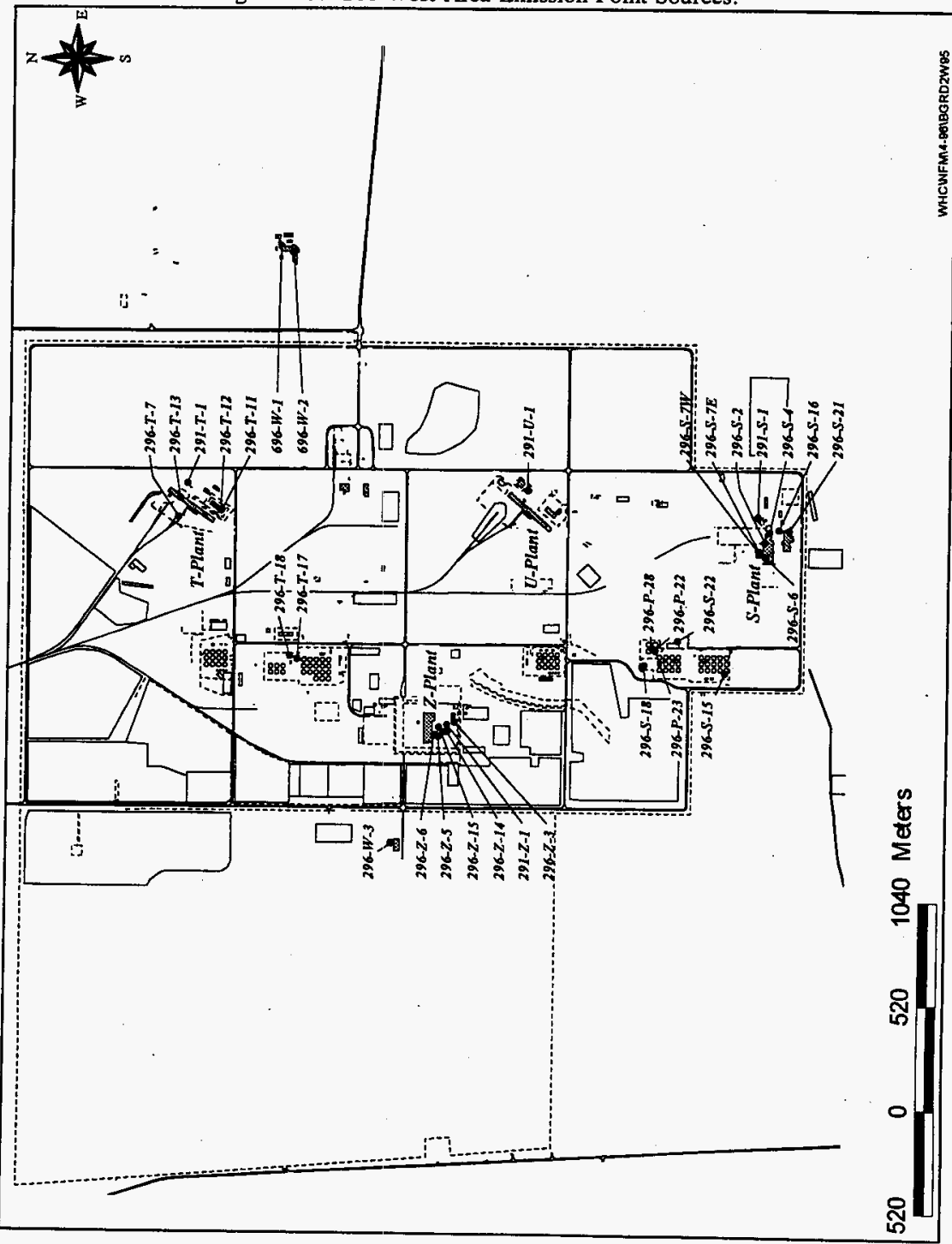


- 296-A-7. This stack exhausts filtered room air from the PUREX west sample gallery and R Cell. Emission monitoring consists of a record sampler.

- 296-A-8. This stack exhausts filtered air from the PUREX Plant Pipe and Operating gallery and the White Room. Emission monitoring consists of a record sampler.

- 296-A-10. This stack exhausts filtered air from storage tunnel No. 2. Emission monitoring consists of a record sampler.

- 296-A-14. This stack exhausts filtered air from the 293-A Off-gas Treatment and Recovery Building (for nitric acid recovery). Emission monitoring consists of a record sampler.

- 296-A-24. This stack exhausts filtered air from $E$ and $F$ Cell vessels to reduce the ammonium nitrate loading on the 291-A-1 filters. Emission monitoring consists of a record sampler.

1.3.3.2 Uranium-TriOxide Plant $\left(\mathrm{UO}_{3}\right.$ Plant). Located in the 200 Area is the UraniumTriOxide $\left(\mathrm{UO}_{3}\right)$ Plant, which formerly produced uranium trioxide from PUREX Plant solutions. In 1972 the $\mathrm{UO}_{3}$ Plant was shut down. Later, it was refurbished to process uranyl nitrate hexahydrate from the PUREX Plant, and was restarted in March 1984. The $\mathrm{UO}_{3}$ Plant operated for the final time in June 1993 processing uranyl nitrate hexahydrate into the more stable compound of uranium trioxide. The plant was deactivated immediately after its final run, in 1993. In December 1994 the facility was transferred from WHC to $\mathrm{BHI}$. $\mathrm{UO}_{3}$ no longer has any point source air emissions, all $\mathrm{UO}_{3}$ stacks were blanked off in late 1994.

1.3.3.3 B Plant and Waste Encapsulation Storage Facility. This facility contains two major operating system areas, B Plant and the Waste Encapsulation Storage Facility (WESF). B Plant was designed and used to separate plutonium from spent nuclear fuel. More recently B Plant was reconfigured to remove ${ }^{137} \mathrm{Cs}$ and ${ }^{90} \mathrm{Sr}$ from high-level liquid waste. At WESF, the ${ }^{137} \mathrm{Cs}$ and ${ }^{90} \mathrm{Sr}$ were converted to solid strontium fluoride and cesium chloride, doubly encapsulated, and placed in water-filled storage basins. WESF is used to store the radioactive strontium and ceșium capsules. The 221-B Building contains radioactive contamination remaining from previous production campaigns that must be properly managed.

- 291-B-1. This is the B Plant main stack, which exhausts filtered air from the B Plant canyon, a process cell in the 212-B Building, and the 224-B Building. Emission monitoring consists of a record sampler, a backup record sampler, and a beta-gamma CAM.

- 296-B-5. This stack exhausts filtered air from the 221-BB Building, which houses the B Plant process condensate and B Plant steam condensate receiver 
tanks. Emission monitoring consists of a record sampler and a beta-gamma CAM.

- 296-B-10. This stack exhausts filtered air from the 225-B Building, which is also known as WESF. Emission monitoring consists of a record sampler and a beta-gamma CAM.

- 296-B-13. This stack exhausts filtered air from the 221-BF Condensate Effluent Discharge Facility, which houses process condensate retention tanks. Emission monitoring consists of a record sampler and a beta-gamma CAM.

1.3.3.4 $A R$ and $C R$ Vaults. The $A R$ and $C R$ Vaults are retention and treatment facilities for high-level radioactive liquid wastes.

- 296-A-12. This stack exhausts filtered air from the 244-AR Vault vessel ventilation system. Emission monitoring consists of a record sampler and a beta-gamma CAM. This stack did not operate in 1995.

- 296-A-13. This stack exhausts filtered air from the 244-AR Vault canyon and cells. Emission monitoring consists of a record sampler and a beta-gamma CAM. This stack did not operate in 1995.

- 296-C-5. This stack exhausts filtered air from the 244-CR Vault Cell and vessel ventilation. Emission monitoring consists of a record sampler and a beta-ganma CAM.

1.3.3.5 200 East Area Tank Farms (excluding the Evaporator). Radioactive waste stored in tank farms consists of sludge and saltcake in single-shell tanks (SSTs) and slurry in double-shell tanks (DSTs).

- 296-A-17 and 296-P-26. These stacks exhaust filtered, incondensable vapors from waste storage tanks in the 241-AY and -AZ Tank Farms. Emission monitoring for each stack consists of a record sampler, two silver-zeolite cartridge samplers for volatile radionuclides, and a beta-gamma CAM. The 296-P-26 stack serves as the backup exhauster for the 296-A-17 stack.

- 296-A-18. This stack exhausts filtered air from the 241-AY-101 tank annulus. Emission monitoring consists of a record sampler. This stack has been inactive since September 1991.

- 296-A-19. When this stack was operational, filtered air was released through it from the 241-AY-102 tank annulus. Emission monitoring consisted of a record sampler. This stack did not operate in 1995. 
- 296-A-20. 241-AZ Tank Annuli Exhaust. This stack exhausts filtered air from the 241-AZ-101 and -102 tank annuli. Emission monitoring consists of a record sampler.

- 296-A-25. This stack exhausts filtered air from the catch tank at the 244-A lift station. Emission monitoring consists of a record sampler and a beta-gamma CAM.

- 296-A-26. This stack exhausts filtered air from the waste unloading room and sump tank at the 204-AR tank. Emission monitoring consists of a record sampler and a beta-gamma CAM.

- 296-A-27. This stack exhausts filtered air from all 241-AW tanks. Emission monitoring consists of a record sampler, two silver-zeolite cartridge samplers for volatile radionuclides, and a beta-gamma CAM.

- 296-A-28. This stack exhausts filtered air from all tank annuli in the 241-AW tank farm. Emission monitoring consists of a record sampler and a beta-gamma CAM.

- 296-A-29. This stack exhausts filtered air from all 241-AN tanks. Emission monitoring consists of a record sampler and a beta-gamma CAM.

- 296-A-30. This stack exhausts filtered air from all tank annuli in the 241-AN tank farm. Emission monitoring consists of a record sampler and a beta-gamma CAM.

- 296-A-40. This stack exhausts filtered air from all 241-AP tanks. Emission monitoring consists of a record sampler, two silver-zeolite cartridge samplers for volatile radionuclides, and a beta-gamma CAM.

- 296-A-41. This stack exhausts filtered air from all tank annuli in the 241-AP tank farm. Emission monitoring consists of a record sampler and a beta-gamma CAM.

- 296-B-28. This stack exhausts filtered air from the 244-BX saltwell receiver tanks and annulus. Emission monitoring consists of a record sampler, a beta-gamma CAM for the tank exhaust, and a beta-gamma CAM for the annulus exhaust.

- 296-P-16. A portable exhauster emits filtered air via this stack from the 241C104, 241C105, and 241C106 tanks. Emission monitoring consists of a record sampler and a beta-gamma CAM. 
- 296-P-17. This portable exhauster emits filtered air from the 241A104, 241A105, and 241A106 tanks. Emission monitoring consists of a record sampler and a beta-gamma CAM. This stack did not operate in 1995.

- 296-P-32, 296-P-33, and 296-P-34. These emission points are the portable exhausters for the Rotary Mode Core Samplers. These emission sources exhaust filtered air from the waste tanks being core sampled. Emissions monitoring consists of a record sampler, a beta-gamma $\mathrm{CM}$, and an alpha CAM.

1.3.3.6 200 East Area Evaporator. The evaporator processed liquid waste in 1995. The evaporator is used to remove most of the water from high-level radioactive liquid waste, leaving a slurry that is pumped back to tank farms.

- 296-A-21. This stack exhausts filtered air from the 242-A EvaporatorCrystallizer Building. Emission monitoring consists of a record sampler, an alpha CAM, and a beta-gamma CAM.

- 296-A-22. This stack exhausts filtered air from the 242-A EvaporatorCrystallizer vessel-ventilation system. Emission monitoring consists of a record sampler and a silver-zeolite cartridge sampler for volatile radionuclides, an alpha CAM, and a beta-gamma CAM.

1.3.3.7 Grout Treatment Facility. This facility solidified low-level waste for disposal in underground vaults. The facility was placed in cold standby in 1993 .

- 296-G-1. When this stack operates air is exhausted from the GTF Air Filtration Module. Emission manitoring consists of a record sampler and a beta-gamma CAM. This stack did not operate in 1995 .

1.3.3.8 209-E Critical Mass Laboratory. The Building was originally designed and used for testing critical mass configurations. Currently the building is being used for office space.

- 296-P-31 (formerly 209-E). This stack exhausts filtered building ventilation air. The particulate alpha and beta radioactivity emissions, from the building exhaust, are sampled with a record sampler.

1.3.3.9 S Plant. S Plant is another name for the Reduction-Oxidation Plant (REDOX), which is the 202-S building. The REDOX Plant was operated as a fuel-reprocessing facility until 1967 when it was shut down.

- 291-S-1. The REDOX main stack exhausts filtered air from the REDOX canyon, vessel ventilation, and treated dissolver off-gas system. Emission monitoring consists of a record sampler and a beta-gamma CAM. 
- 296-S-2. This stack exhausts filtered air from REDOX north and south sample galleries, hoods, and product removal cage. Emission monitoring consists of a record sampler.

- 296-S-4. This stack exhausts filtered air from the REDOX decontamination room and regulated shop and unfiltered air from the regulated tool room, low-level decontamination sink, and special work permit lobby. Emission monitoring consists of a record sampler.

- 296-S-6. This stack exhausts air from the REDOX Plant, the silo gallery, an organic feed tank, and a sample elevator. Emission monitoring consists of a record sampler.

- 296-S-7W and 296-S-7E. These stacks exhaust filtered air from the 233-S REDOX Product building, the REDOX plutonium-processing greenhouse, and process vessel ventilation and load-out area. Emission monitoring for each stack consists of a record sampler and an alpha CAM. The 296-S-7E stack serves as the backup exhauster for the 296-S-7W.

1.3.3.10 T Plant. T Plant is one of the original fuel reprocessing facilities. The last fuel reprocessed there was in 1956. The 221-T Building is currently used to treat, store, repackage, sample, and verify wastes. The 221-T Building also is used for decontaminating and repairing equipment.

- 291-T-1. This stack exhausts filtered air from 221-T canyon and process ventilation. Emission monitoring consists of a record sampler, a beta-gamma CAM, and an alpha CAM.

- 296-T-13. This stack exhausts filtered air from the 221-T Building and canyon ventilation. Emission monitoring consists of a record sampler and a beta-gamma CAM.

- 296-T-7. This stack exhausts filtered air from the 2706 Building only while decontamination activities are being performed, the remaining times it is turned off. Emission monitoring consists of a record sampler and beta-gamma CAM.

1.3.3.11 TRUSAF. The 224-T Building was originally used to purify plutonium nitrate by the lanthanum fluoride process. After phase-out of the bismuth phosphate plants, the lanthanum fluoride process was no longer needed and the facility remained inactive until the early 1970 's. At that time, the 224-T Building was modified for storage of plutonium scrap in liquid and solid forms. In 1984 the facility was selected to house the transuranic waste storage and assay (TRUSAF) operation. The TRUSAF operation involves the analysis of transuranic waste to verify compliance with the Waste Isolation Pilot Plant (WIPP) waste 
acceptance criteria. Those containers meeting WIPP acceptance criteria are stored at 224-T pending shipment to the WIPP.

- 296-T-11. This stack exhausts filtered air from the 224-T Building ventilation through the east part of the plenum. Emission monitoring consists of a record sampler.

- 296-T-12. This stack exhausts filtered air from the 224-T Building ventilation through the west part of the plenum. Emission monitoring consists of a record sampler.

1.3.3.12 U Plant. U Plant was constructed as a fuel reprocessing plant but was never used for that purpose. Instead, it was used to recover uranium from bismuth-phosphate waste and high-level radioactive wastes from tank farms. Currently, it is a retired facility with a few offices and shops still in use.

- 291-U-1. This stack exhausts filtered air from U Plant and 221-U canyon ventilation. Emission monitoring consists of a record sampler and a beta-gamma CAM.

1.3.3.13 Plutonium Finishing Plant (PFP). PFP was constructed to produce plutonium metal from recovered nitrate and plutonium nitrate received from the PUREX Plant. PFP also recovered plutonium, in the form of plutonium nitrate, from plutonium scrap. PFP's current mission is to stabilize and store existing inventory of plutonium compounds.

- 291-Z-1. This stack exhausts filtered air from the 234-5Z, 236-Z, and 242-Z Buildings. Emission monitoring consists of a record sampler and an alpha CAM.

- 296-Z-3. This stack exhausts filtered air from $241-Z$ vault sump and vessel ventilation. Emission monitoring consists of a record sampler and an alpha CAM.

- 296-Z-5. This stack exhausts filtered air from 2736-ZB, the shipping and receiving building. Emission monitoring consists of a record sampler and an alpha CAM.

- 296-Z-6. This stack exhausts filtered air from the 2736-Z Building and its plutonium storage vault ventilation system. Emission monitoring consists of a record sampler and two alpha CAMs. One alpha CAM is located on each of the two ducts feeding into this stack.

- 296-Z-14. This stack exhausts filtered air from the 232-Z Incinerator Building. Emission monitoring consists of a record sampler and two alpha CAMs. 
- 296-Z-15. This stack exhausts filtered air from the 243-Z Low Level Waste Treatment Facility. Emissions monitoring consists of a record sampler.

1.3.3.14 200 West Area Tank Farms (Excluding Evaporators). Radioactive waste stored in tank farms consists of sludge and saltcake in SSTs and liquid and slurry in DSTs.

- 296-P-22. This stack exhausts filtered air from annuli in the 241-SY-101, 102, and -103 tanks. Emission monitoring consists of a record sampler and a beta-gamma CAM.

- 296-P-23 (296-P-28 backup stack). This stack exhausts filtered air from the 241-SY-101, -102, and -103 tanks. Emission monitoring consists of a record sampler and a beta-gamma CAM.

- 296-S-15. This stack exhausts filtered air from tanks $241-S X-107$ through -112 and 241-SX-114. Emission monitoring consists of a record sampler and a beta-gamma CAM.

- 296-S-22. This stack exhausts filtered air from the 244-S saltwell receiver tank and annulus. Emission monitoring consists of a record sampler, an alpha CAM, and a beta-gamma CAM.

- 296-T-18. This stack exhausts filtered air from the 244-TX saltwell receiver tank and annulus. Emission monitoring consists of a record sampler, an alpha CAM, and a beta-gamma CAM.

1.3.3.15 200 West Area Evaporators. The evaporators are designed to remove most of the water from radioactive liquid waste; the resulting slurry is then routed to tank farms for storage. The 242-T Evaporator-Crystallizer has been deactivated and has been inoperable since 1986. The 242-S Evaporator-Crystallizer did not operate in 1995, but is on standby.

- 296-S-18. This stack exhausts filtered air from the 242-S EvaporatorCrystallizer Building. Emission monitoring consists of a record sampler and a beta-gamma CAM.

- 296-T-17. This stack exhausts filtered air from the 242-T EvaporatorCrystallizer and cold-cell ventilation. Emission monitoring consists of a record sampler, an alpha CAM, and a beta-gamma CAM.

1.3.3.16 222-S Analytical Laboratories. The 222-S Analytical Laboratories provide chemical and radiochemical analytical support for WHC, including environmental and effluent sample analysis, research and development, and tank farm waste characterization. 
- 296-S-16. This stack exhausts filtered air from the 219-S Building and waste tanks. Emission monitoring consists of a record sampler.

- 296-S-21. This stack exhausts filtered air from 222-S Laboratory hoods, gloveboxes, hot-cells, and room ventilation system. Emission monitoring consists of a record sampler, an alpha CAM, and a beta-gamma CAM.

1.3.3.17 Waste Sampling and Characterization Facility (WSCF). The WSCF laboratory provides low-level radiological and chemical analyses on various types of samples and sample medias. The majority of the analyzed samples are used to determine compliance with the requirements of environmental regulations and U.S. Department of Energy (DOE) Orders. Note that WSCF is technically located in the 600 Area, and is immediately outside of the 200 West Area's perimeter. It is included in the section on for 200 West Area, since its releases are modelled from the 200 West Area.

- 696-W-1. This stack exhausts filtered air from the analytical laboratory located on the main floor of the 6266 building. Emission monitoring consists of a record air sampler.

- 696-W-2. This stack exhausts filtered air from the Nuclear Spectroscopy Laboratory located in the basement of the 6266 building. Emission monitoring consists of a record sampler.

1.3.3.18 Waste Verification \& Sampling Facility. The facility is used to verify the contents of drums received from generators. Because of limited use, it was transferred to West Tank Farms in 1995.

- 296-W-3. This exhaust discharges filtered air from the 213-W building. Emission monitoring consists of a record sampler and a beta-gamma CAM.

\subsubsection{Area Facilities}

The 300 Area consists primarily of laboratories, research facilities, and a steam plant. Emission points in the 300 Area are shown in Figure 1-6.

1.3.4.1 303-C Materials Evaluation Laboratory. The building is used primarily for storage of special nuclear materials in sealed containers.

- EP-303C-01-S. This stack exhausts filtered building ventilation air. The exhaust is sampled for particulate alpha and beta radioactivity.

1.3.4.2 304 Uranium Concretion Facility. In 1994, this facility was operated in a limited capacity for preparing radioactively contaminated sludges and fines for disposal or storage. 


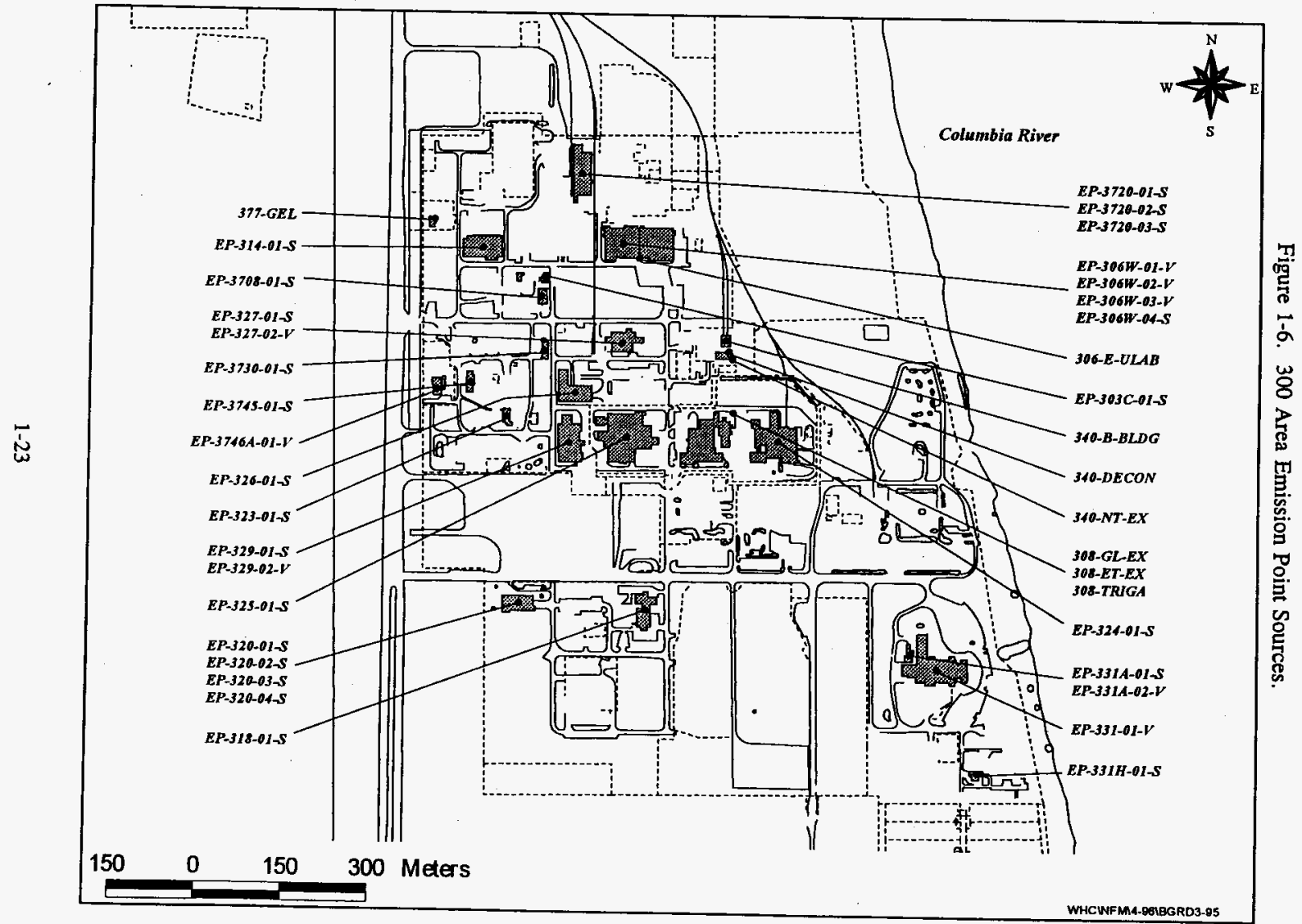

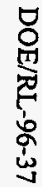


Activities performed at this facility included mixing radioactive contaminated matrices with cement and water. The facility is currently in transition to closure under RCRA regulations. Its stack, 304-CON-EX, was sealed off in 1995 and no longer has an emission point.

1.3.4.3 306-E Metal Fabrication Development Building. This building is in use for materials testing and fabrication. In the past, this building housed three separate operations; (1) a large, high-bay used for unfueled test article fabrication for FFTF, (2) nondestructive, radiography examination in a series of shielded cells, and (3) a small depleted-uraniumpowder laboratory used to fabricate insulator pellets for the FFTF fuel pins. In August 1995 , this facility was shutdown and has since been stabilized, changing its radiological status to a fixed contamination area.

- 306-E-ULAB. This stack exhausts filtered air from the Uranium Oxide Laboratories in rooms 158 and 159 , which no longer contains loose contamination. Emission monitoring consists of a record sampler.

1.3.4.4 306-W Materials Development Laboratory. The building contains shops and laboratory facilities for metal-working and ceramic studies.

- EP-306W-01-V. This vent exhausts filtered building ventilation air. The exhaust is sampled for particulate alpha and beta radioactivity.

- EP-306W-02-V. This vent exhausts filtered building ventilation air. The exhaust is sampled for particulate alpha and beta radioactivity.

- EP-306W-03-V. This vent exhausts filtered building ventilation air. The exhaust is sampled for particulate alpha and beta radioactivity.

- EP-306W-04-S. This vent exhausts filtered building ventilation air from the northwest portion of the building. The exhaust is sampled for particulate alpha and beta radioactivity.

1.3.4.5 308 Fuels Development Laboratory. The 308 Laboratory was used for fabricating mixed-oxide fuel pins used by FFTF. The facility houses a shutdown swimming-pool-type Test Reactor and Isotope Production reactor by General Atomics (TRIGA) that was used in neutron radiography of the completed pins. The 308 Building is in transition to shutdown.

- 308-TRIGA. This stack discharges filtered air from the reactor hall housing the TRIGA reactor. The reactor, however, is permanently shut down and partially defueled. Emission monitoring consists of a particulate record sampler.

1.3.4.5 309 Plutonium Recycle Test Reactor. The 309 Building's containment dome and support facilities once housed the Plutonium Recycle Test Reactor (PRTR). In 1962, the Plutonium Recycle Critical Facility (PRCF) was added to support the PRTR operations. By 
1975 , the PRTR was deactivated, all of the fuel removed from the building, and the fuel storage basin decontaminated. In the mid 1980's, an extensive cleanout effort removed most of the process equipment and vessels. The ground level of the containment dome is currently being used an assembly shop and the remainder of the building is used as office space.

- 309-PRTR. The 309 Building's primary stack ventilates what used to be the PRTR facility. Periodic confirmatory measurements are used to confirm that emissions are at or below acceptable levels.

- 309-RTF-EX. 309 Building rupture loop annex exhaust stack. Periodic confirmatory measurements are used to confirm that emissions are at or below acceptable levels.

1.3.4.6 340 Complex. The 340 Complex houses the radioactive liquid waste and solid waste handling operations for the 300 Area. The 340-A Building contains six aboveground storage tanks for radioactive liquid waste. The east side of the $340-\mathrm{B}$ building is a railway car loadout facility for shipping liquid waste to the 200 Areas. The west side of the $340-\mathrm{B}$ building is a storage area for non-radioactive and radioactive solid waste.

- 340-NT-EX. This stack exhausts filtered air from the 340 Building vault, the 340 Building vault tanks, and the 340-A Building waste tanks. Emission monitoring consists of a particulate record sampler, an alpha CAM, a betagamma CAM, and a record sampler for volatile ${ }^{131} \mathbf{I}$.

- 340-B-BLDG. This stack exhausts filtered air from the 340-B East Building. The stack exhaust system operates when railway cars are housed within the facility. Emissions monitoring consists of a particulate record sampler.

- 340-DECON. This stack exhausts air from the 340 Facility truck lock, operator's office, change rooms, decontamination area, and sampling hood. Emissions monitoring consist of a particulate record sampler.

1.3.4.7 314 Engineering Development Laboratory. The building contains high-bay facilities and laboratories for engineering studies.

- EP-314-01-S. This stack exhausts filtered building ventilation air from chemistry laboratories on the north side of the building. The exhaust is sampled for particulate alpha and beta radioactivity.

1.3.4.8 318 Radiological Sciences Laboratory. The building contains facilities for calibrating radiation survey instruments and processing personnel dosimeters.

- EP-318-01-S. This stack exhausts unfiltered building ventilation air. The exhaust is sampled for particulate alpha and beta radioactivity. 
1.3.4.9 320 Physical Sciences Laboratory. The building contains environmental radiochemistry laboratories.

- EP-320-01-S. This stack exhausts filtered building ventilation air. The exhaust is sampled for particulate alpha and beta radioactivity.

- EP-320-02-S. This stack exhausts filtered chemistry hoods. The exhaust is sampled for particulate alpha and beta radioactivity.

- EP-320-03-S. This stack exhausts filtered chemistry hoods. The exhaust is sampled for particulate alpha and beta radioactivity.

- EP-320-04-S. This stack exhausts filtered chemistry hoods. The exhaust is sampled for particulate alpha and beta radioactivity.

1.3.4.10 323 Mechanical Properties Laboratory. The building contains facilities for investigating structural properties of irradiated materials.

- EP-323-01-S. This stack exhausts filtered building ventilation air. The exhaust is sampled for particulate alpha and beta radioactivity.

1.3.4.11 324 Waste Technology Engineering Laboratory. The building contains laboratories for performing chemical and process development activities.

- EP-324-01-S. This stack exhausts filtered building air. The exhaust is sampled for particulate radioactivity (total alpha, total beta, ${ }^{90} \mathrm{Sr},{ }^{238} \mathrm{Pu}$, ${ }^{239.240} \mathrm{Pu}$, and gamma emitting radionuclides) and tritium (HTO and HT).

1.3.4.12 325 Applied Chemistry Laboratory. The building contains radiochemistry laboratories.

- EP-325-01-S. This stack exhausts filtered building air. The exhaust is sampled for particulate radioactivity (total alpha, total beta, ${ }^{90} \mathrm{Sr},{ }^{238} \mathrm{Pu}$, ${ }^{239.240} \mathrm{Pu}$, and gamma emitting radionuclides) and tritium (HTO and HT).

1.3.4.13 326 Materials Sciences Laboratory. The building contains laboratories and equipment for studies of metallurgical, chemical, and physical behavior of reactor components and fuel materials.

- EP-326-01-S. This stack exhausts filtered building air. The exhaust is sampled for particulate radioactivity (total alpha and total beta).

1.3.4.14 327 Post Irradiation Testing Laboratory. The building contains hot-cells for examining and testing irradiated materials. 
- EP-327-01-S. This stack exhausts filtered building air. The exhaust is sampled for particulate radioactivity (total alpha, total beta, ${ }^{90} \mathrm{Sr},{ }^{238} \mathrm{Pu}$, ${ }^{239.240} \mathrm{Pu},{ }^{241} \mathrm{Am}$, and gamma emitting radionuclides) and radon $\left({ }^{220} \mathrm{Rn}\right.$ and ${ }^{222} \mathrm{Rn}$ ).

- EP-327-02-V. This roof vent exhausts filtered air from the decontamination cell. The exhaust is sampled for particulate radioactivity (total alpha, total beta, ${ }^{90} \mathrm{Sr},{ }^{238} \mathrm{Pu},{ }^{239,240} \mathrm{Pu},{ }^{241} \mathrm{Am}$, and gamma emitting radionuclides).

1.3.4.15 329 Chemical Sciences Laboratory. The building contains chemistry laboratories for radioanalytical studies, environmental radionuclide studies, and radiation detection instrumentation development.

- EP-329-01-S. This vent exhausts filtered building ventilation air. The exhaust is sampled for particulate alpha and beta radioactivity.

- EP-329-02-V. This vent exhausts filtered building ventilation air. The exhaust is sampled for particulate alpha and beta radioactivity.

1.3.4.16 329 Neutron Multiplier Facility. The building contains a neutron multiplier source for activation analysis and on-line spectrometry of fission products. The emission point for this facility was removed in November of 1994.

1.3.4.17 331 Life Sciences Laboratory. The building contains facilities for biological and ecological research studies.

- EP-331-01-V. This vent exhausts filtered building ventilation air. The exhaust is sampled for particulate alpha and beta activity.

- EP-331A-01-S. This stack exhausts filtered building ventilation air. The exhaust is sampled for particulate alpha and beta radioactivity.

- EP-331A-02-V. This vent exhausts filtered building ventilation air from the liquid waste transfer room. The room contains facilities for transferring and storing radioactive liquid waste used in biological research studies. The exhaust is sampled for particulate alpha and beta radioactivity.

1.3.4.18 331-H Aerosol Wind Tunnel Research Facility. The building contains a wind tunnel for atmospheric pollutant deposition studies. There are no unsealed radionuclides in use in the facility at this time.

- EP-331H-01-S. This stack exhausts filtered building ventilation air. The exhaust is sampled for particulate alpha and beta radioactivity. 
1.3.4.19 377 Geotechnical Engineering Laboratory. This facility was formerly known as the 377 Steam Generator Examination Facility. In 1993 the facility was renamed the 377 Geotechnical Engineering Laboratory to reflect the change in operations. The stack name was also changed from $377-$ SGEF to 377 -GEL. The facility was formerly used for studying a steam generator that had been removed from a nuclear power plant. The steam generator was removed before 1990 and the facility was decontaminated. In 1991 and 1992, the facility was modified for use as a physical properties testing laboratory for radioactively contaminated soils and began laboratory operation in 1993.

- 377-GEL. This stack exhausts filtered building ventilation air. The exhaust is sampled for particulate alpha and beta radioactivity.

1.3.4.20 3708 Radiation Measurements Laboratory. The building contains facilities for measuring small amounts of radionuclides, performing calibration studies, and performing leach studies with vitrified radioactive waste.

- EP-3708-01-S. This stack exhausts filtered building ventilation air. The exhaust is sampled for particulate alpha and beta radioactivity.

1.3.4.21 3720 Chemical and Metal Sciences Laboratory. The building contains low-level radiochemistry laboratories and a counting room.

- EP-3720-01-S. This stack exhausts filtered building air. The exhaust is sampled for particulate radioactivity (total alpha and total beta).

- EP-3720-02-S. This stack exhausts filtered building air from the north-annex low-level radiochemistry laboratories. The exhaust is sampled for particulate radioactivity (total alpha and total beta).

- EP-3720-03-S. This stack exhausts filtered building air from the south-end laboratories. This region of the building contains a counting room and lowlevel radiochemistry laboratories. The exhaust is sampled for particulate radioactivity (total alpha and total beta).

1.3.4.22 3730 Gamma Irradiation Facility. The building contains a hot-cell for metallurgical studies of specimens of irradiated metals.

- EP-3730-01-S. This stack exhausts filtered building ventilation air. The exhaust is sampled for particulate radioactivity (total alpha and total beta).

1.3.4.23 3745 Radiological Calibrations and Standards Facility. The building contains a counting room for measuring facility ventilation and exhaust samples.

- EP-3745-01-S. This stack exhausts filtered building ventilation air. The exhaust is sampled for particulate radioactivity (total alpha and total beta). 
1.3.4.24 3746-A Radiological Physics Laboratory. The building contains facilities for low-level radiological physics studies.

- EP-3746A-01-V. This vent exhausts filtered building ventilation air. The exhaust is sampled for particulate radioactivity (total alpha and total beta).

\subsubsection{Area Facilities}

The 400 Area consists of the FFTF, the Maintenance and Storage Facility (MASF), and the Fuels Materials Examination Facility. Emission points in the 400 Area are shown in Figure 1-7.

1.3.5.1 Fast Flux Test Facility. FFTF, located in the 400 Area, is a 400 -megawatt thermal, sodium-cooled, low-pressure, high-temperature reactor plant. It was used for irradiation testing of breeder reactor fuels and materials.

- FFTF-RE-SB. Lower Reactor Service Building (RSB). This exhaust discharges unfiltered air from the lower level of the RSB. Emission monitoring consists of a particulate record sampler, a beta CAM, a noble gas monitor, and a record sampler for volatile ${ }^{131} I$.

- FFTF-CB-EX. Combined exhaust. This stack exhausts normally unfiltered air from the reactor containment and gases from the argon processing system. Standby particulate filters are automatically dampered into the system if airborne radioactive particulate concentrations exceed administrative limits. Emission monitoring consists of an particulate record sampler, a beta CAM, a noble gas monitor, a tritium (HTO) record sampler, and a record sampler for volatile ${ }^{131}$.

- FFTF-HT-TR. Heat Transport System South. This exhaust discharges normally unfiltered air from portions of FFTF that are exterior to the containment. Standby particulate filters are automatically dampered into the system if airborne radioactive particulate concentrations exceed administrative limits. Emission monitoring consists of a record sampler, a beta CAM, and a noble gas monitor.

1.3.5.2 437 MASF. The Maintenance and Storage Facility (MASF) is a multipurpose service center supporting the specialized maintenance and storage requirements of FFTF. The MASF provides the capability for sodium film removal, decontamination, repair, and storage of nonfueled components and hardware for FFTF.

- 437-MN\&ST. This exhaust discharges filtered air from the MASF. Emission monitoring consists of a record sampler and a beta CAM. 
DOE/RL-96-37

Figure 1-7. 400 Area Emission Point Sources.

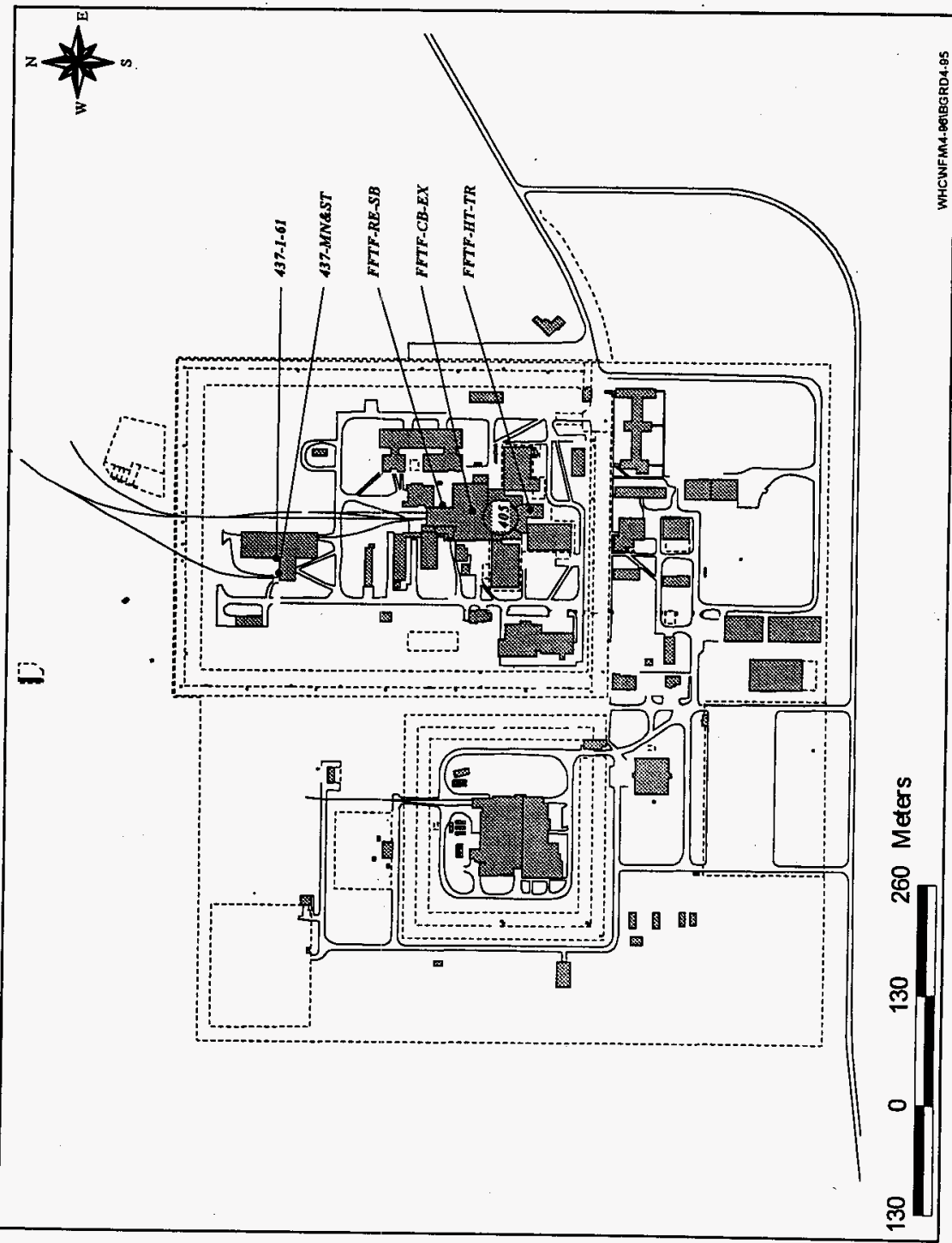


- 437-1-61. This exhaust discharges filtered air from the MASF. Emission monitoring consists of a record sampler and a beta CAM.

\subsubsection{Area Facilities}

Emission points in the 600 Area are shown in Figure 1-8.

1.3.6.1 6652-H Ecology Laboratory. This laboratory is located on the Fitzner-Eberhardt Arid Land Ecology Reserve. The building contains facilities for ecology studies.

- EP-6652H-01-V. This vent exhausted filtered building ventilation air. The exhaust was sampled for particulate alpha and beta radioactivity. This point source was sealed off and its fans taken out of service on 1/30/96. 
DOE/RL-96-37

Figure 1-8. 600 Area Emission Point Sources.

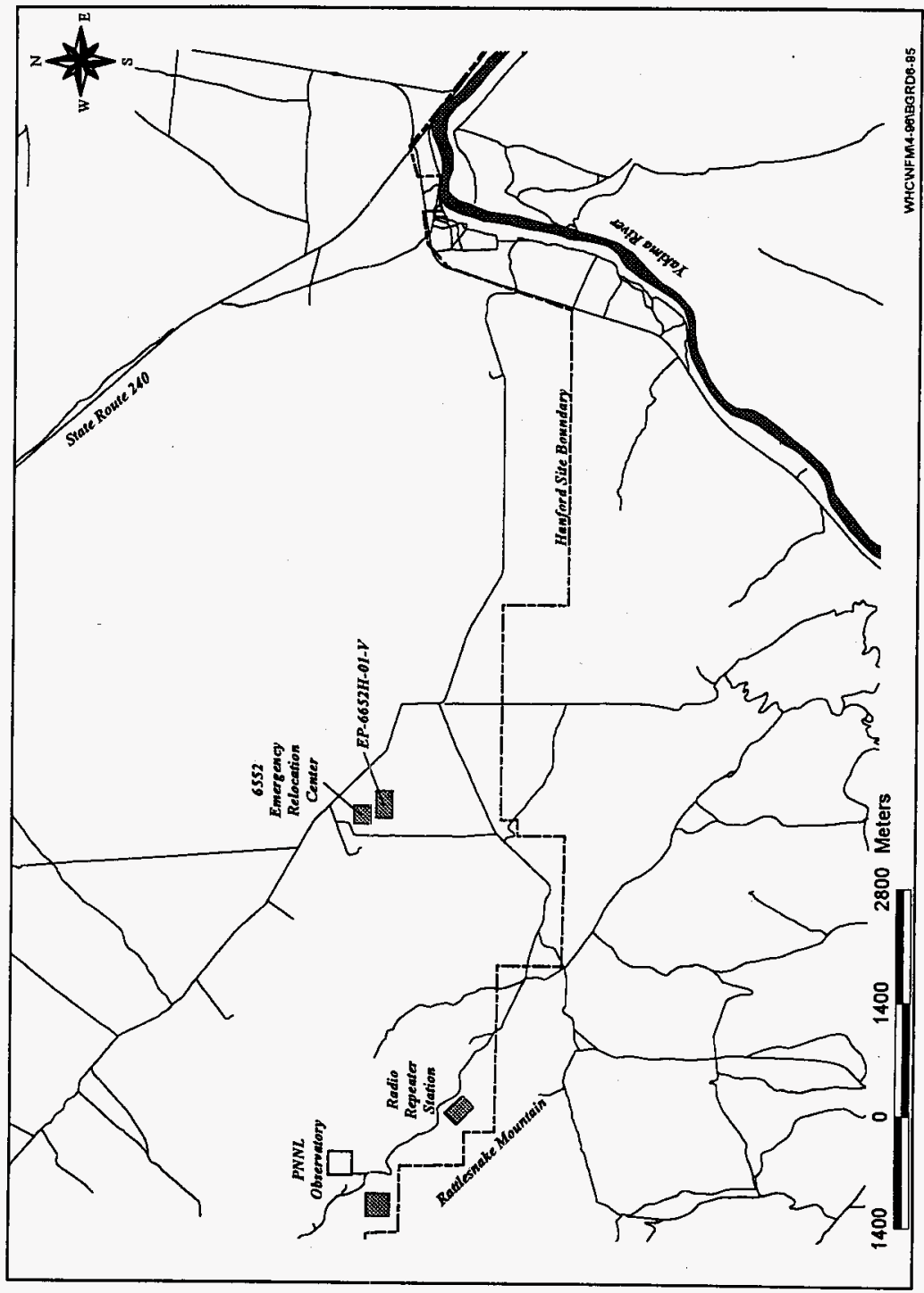




\subsection{RADIONUCLIDE AIR EMISSIONS DATA ON POINT SOURCES}

Data on radionuclide emissions released during 1995 from point sources (e.g. stacks, vents, etc...), described in Section 1.3, are shown in Tables 2-1 and 2-2. The criteria for reporting emissions are discussed in Section 1.3.1. Section 1.3.1 also provides the basis for determining when an emissions source shall be included in this report.

Table 2-1 displays source data on the Hanford Site's 25 major point sources. A point source is classified as major if its emissions, with all treatment controls removed, have the potential to exceed $0.1 \mathrm{mrem} / \mathrm{y}$ effective dose equivalent (EDE), at the nearest residence.

A nearest residence is a known habitable location not on the Hanford Site (dwelling, business, school, or office) that is nearest to a point source representative of a collective operating area such as the 100 Areas. The maximally exposed individual (MEI) for the Hanford Site differs from the nearest receptor in that the sitewide MEI is a hypothetical member of the public residing near the Hanford Site who, by virtue of location and living habits, could receive the highest possible radiation dose from all radionuclide emissions from the Hanford Site. Historically, the MEI has been an individual living in the Ringold, Washington area. In the last three years the MEI has been calculated to be a resident of a farm east of the 300 Area.

The point sources that do not meet the criteria for being major sources are referred to as minor point sources. The minor point sources are included in Table 2-2, which contains data on an additional 96 emission points. These point sources are grouped according to the operating areas and facilities they are located in. A point source is considered minor when its potential maximum emissions with all treatment controls hypothetically removed cannot cause a dose at the nearest residence to exceed $0.1 \mathrm{mrem} / \mathrm{y}$ EDE.

Each point source listed has information on discharge height, distance to the nearest offsite residence, type and efficiency of any emission control, and the 1995 releases by radionuclide or type of radioactivity. The efficiencies for the various emission control devices, used at Hanford, are specified in the footnotes of Tables 2-1 and 2-2.

Distances to the nearest offsite dwelling, business, school, or office from reference points for emission sources on the Hanford Site are listed in Table 2-3. Distances to the nearest farms producing milk, vegetables, and meat are also included in Table 2-3. For dose modelling, the point sources are grouped by operating area, resulting in a single distance applicable to all point sources in that area. Reporting these distances is a requirement of 40 CFR 61, Subpart H (EPA 1989).

The distances to the nearest receptors, and not the sitewide MEI, were used in determining whether a point source was major or minor (WHC 1991). These distances, however, were not used in calculating the EDEs reported in this document. Instead, the distances from each operating area to the MEI location, a farm east of the 300 Area, were 
used in the EDE calculations. The MEI location represents the receptor point for the maximum dose from the total combined emissions of all Hanford Site emission sources, not just those in individual operating areas. The nearest school to the MEI receptor point is about $4.0 \mathrm{mi}(6.4 \mathrm{~km})$ northeast.

An example, of the difference between the two sets of distances, is represented by the 200 West Area. The distance from 200 West Area emission points to the nearest residence is $15 \mathrm{mi}(24 \mathrm{~km})$ east. But the distance from 200 West Area emission points to the Hanford Site MEI location, on Sagemore Road, is $22 \mathrm{mi}(35 \mathrm{~km})$ southeast. 
Table 2-1

\section{Hanford Site Radionuclide Air Emissions Data}

For Major Point Sources.

(major point sources have the potential of $>0.1 \mathrm{mrem} / \mathrm{y}$ EDE to nearest offsite resident)

\begin{tabular}{|c|c|c|c|c|c|c|c|}
\hline $\begin{array}{c}\text { Source } \mathrm{IO}^{\mathrm{b}} \\
\text { (facility/building) } \\
\text { [contractor] }\end{array}$ & $\begin{array}{c}\text { Discharge } \\
\text { Height } \\
\text { (m) }\end{array}$ & $\begin{array}{l}\text { Emission } \\
\text { Control } \\
\text { (stages) }\end{array}$ & $\begin{array}{l}\text { Total } \\
\text { Flow } \\
\left(\mathrm{w}^{3}\right)\end{array}$ & $\begin{array}{c}\text { Annual } \\
\text { Average } \\
\text { Flowrate } \\
\left(\mathrm{m}^{3} / \mathrm{s}\right)\end{array}$ & Radionuclide ${ }^{d}$ & $\begin{array}{c}\text { Annual } \\
\text { Emissions } \\
\text { (Ci) }^{\mathbf{e}}\end{array}$ & $\begin{array}{c}\mathbf{E D E}^{\mathbf{f}} \\
\text { for } \\
\text { MEI } \\
\text { (mrem/y) }\end{array}$ \\
\hline \multicolumn{8}{|c|}{200 East Area Point Sources } \\
\hline $\begin{array}{l}291-\mathrm{A}-1 \\
\text { (PUREX) } \\
\text { [WHC] }\end{array}$ & 61.0 & HEPA (3) & $1.6 \mathrm{E}+09$ & 50.7 & \begin{tabular}{|l}
${ }^{90} \mathrm{Sr}$ \\
${ }^{106} \mathrm{Ru}$ \\
${ }^{113} \mathrm{Sn}$ \\
${ }^{125} \mathrm{Sb}$ \\
${ }^{129} \mathrm{I}$ \\
${ }^{134} \mathrm{Cs}$ \\
${ }^{137} \mathrm{Cs}$ \\
${ }^{152} \mathrm{Eu}$ \\
${ }^{154} \mathrm{Eu}$ \\
${ }^{153} \mathrm{Eu}$ \\
${ }^{238} \mathrm{Pu}$ \\
${ }^{239.240} \mathrm{Pu}$ \\
${ }^{241} \mathrm{Pu}$ \\
${ }^{241} \mathrm{Am}$
\end{tabular} & $\begin{array}{c}4.2 \mathrm{E}-05 \\
\mathrm{ND} \\
\mathrm{ND} \\
7.4 \mathrm{E}-06 \\
8.6 \mathrm{E}-03 \\
\mathrm{ND} \\
1.0 \mathrm{E}-04 \\
3.5 \mathrm{E}-07 \\
3.2 \mathrm{E}-07 \\
9.4 \mathrm{E}-08 \\
5.7 \mathrm{E}-07 \\
4.5 \mathrm{E}-06 \\
1.1 \mathrm{E}-04 \\
1.6 \mathrm{E}-05\end{array}$ & $\begin{array}{l}1.2 \mathrm{E}-06 \\
5.0 \mathrm{E}-08 \\
1.8 \mathrm{E}-03 \\
6.1 \mathrm{E}-06 \\
2.2 \mathrm{E}-08 \\
1.6 \mathrm{E}-08 \\
1.9 \mathrm{E}-10 \\
9.8 \mathrm{E}-07 \\
8.2 \mathrm{E}-06 \\
3.1 \mathrm{E}-06 \\
4.4 \mathrm{E}-05\end{array}$ \\
\hline $\begin{array}{l}296-A-1 \\
\text { (PUREX) } \\
{[\text { WHC] }}\end{array}$ & 20.7 & HEPA (3) & $5.9 \mathrm{E}+07$ & 1.9 & $\begin{array}{l}{ }^{238} \mathrm{Pu} \\
{ }^{239.240} \mathrm{Pu} \\
{ }^{241} \mathrm{Pu} \\
{ }^{241} \mathrm{Am} \\
\text { total beta }\end{array}$ & $\begin{array}{l}1.1 \mathrm{E}-07 \\
1.8 \mathrm{E}-06 \\
9.4 \mathrm{E}-06 \\
5.4 \mathrm{E}-07 \\
1.3 \mathrm{E}-07\end{array}$ & $\begin{array}{l}1.9 \mathrm{E}-07 \\
3.3 \mathrm{E}-06 \\
2.6 \mathrm{E}-07 \\
1.5 \mathrm{E}-06 \\
3.6 \mathrm{E}-09\end{array}$ \\
\hline $\begin{array}{l}291-\mathrm{B}-1 \\
\text { (B Plant) } \\
\text { [WHC] }\end{array}$ & 61.0 & HEPA (2) & $5.8 \mathrm{E}+08$ & 18.3 & $\begin{array}{l}{ }^{90} \mathrm{Sr} \\
{ }^{106} \mathrm{Ru} \\
{ }^{13} \mathrm{Sn} \\
{ }^{125} \mathrm{Sb} \\
{ }^{134} \mathrm{Cs} \\
{ }^{137} \mathrm{Cs} \\
{ }^{152} \mathrm{Eu} \\
{ }^{154} \mathrm{Eu} \\
{ }^{155} \mathrm{Eu} \\
{ }^{238} \mathrm{Pu} \\
{ }^{139,240} \mathrm{Pu} \\
{ }^{241} \mathrm{Am}\end{array}$ & $\begin{array}{c}8.7 \mathrm{E}-06 \\
\mathrm{ND} \\
\mathrm{ND} \\
4.1 \mathrm{E}-08 \\
\mathrm{ND} \\
2.1 \mathrm{E}-04 \\
\mathrm{ND} \\
\mathrm{ND} \\
7.0 \mathrm{E}-08 \\
9.0 \mathrm{E}-09 \\
9.5 \mathrm{E}-07 \\
6.4 \mathrm{E}-08\end{array}$ & $\begin{array}{l}2.4 \mathrm{E}-07 \\
2.7 \mathrm{E}-10 \\
1.3 \mathrm{E}-05 \\
1.4 \mathrm{E}-10 \\
1.5 \mathrm{E}-08 \\
1.7 \mathrm{E}-06 \\
1.8 \mathrm{E}-07\end{array}$ \\
\hline
\end{tabular}


Table 2-1

(7 sheets)

\begin{tabular}{|c|c|c|c|c|c|c|c|}
\hline \multicolumn{8}{|c|}{$\begin{array}{l}1995 \text { Hanford Site Radionuclide Air Emissions Data } \\
\text { For Major Point Sources. } \\
\text { (major point sources have the potential of }>0.1 \mathrm{mrem} / \mathrm{y} \text { EDE to nearest offsite resident) }\end{array}$} \\
\hline $\begin{array}{c}\text { Source } \mathbf{I D}^{\mathbf{b}} \\
\text { (facility/building) } \\
\text { [contractor] }\end{array}$ & $\begin{array}{c}\text { Discharge } \\
\text { Height } \\
\text { (m) }\end{array}$ & $\begin{array}{l}\text { Emission } \\
\text { Control } \\
\text { (stages) }\end{array}$ & $\begin{array}{l}\text { Total } \\
\text { Flow } \\
\left(\mathbf{m}^{3}\right)\end{array}$ & $\begin{array}{c}\text { Annual } \\
\text { Average } \\
\text { Flowrate } \\
\left(\mathbf{m}^{3} / \mathbf{s}\right)\end{array}$ & Radionuctide $^{d}$ & $\begin{array}{c}\text { Annual } \\
\text { Emissions } \\
(C i)^{e}\end{array}$ & $\begin{array}{c}\text { EDE }^{\text {f }} \\
\text { for } \\
\text { MEI } \\
(\mathbf{m r e m} / \mathbf{y})\end{array}$ \\
\hline $\begin{array}{l}296-\mathrm{A}-22 \\
\text { (242-A Evaporator) } \\
\text { [WHC] }\end{array}$ & 18.6 & HEPA (2) & $9.6 \mathrm{E}+06$ & 0.3 & $\begin{array}{l}{ }^{90} \mathrm{Sr} \\
{ }^{106} \mathrm{Ru} \\
{ }^{13} \mathrm{Sn} \\
{ }^{125} \mathrm{Sb} \\
{ }^{129} \mathrm{I} \\
{ }^{134} \mathrm{Cs} \\
{ }^{137} \mathrm{Cs} \\
{ }^{152} \mathrm{Eu} \\
{ }^{154} \mathrm{Eu} \\
{ }^{155} \mathrm{Eu} \\
{ }^{238} \mathrm{Pu} \\
{ }^{239}, 240 \mathrm{Pu} \\
{ }^{241} \mathrm{Am}\end{array}$ & 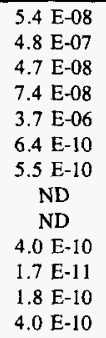 & $\begin{array}{l}1.5 \text { E-09 } \\
1.6 \text { E-09 } \\
1.4 \text { E-11 } \\
5.0 \text { E-10 } \\
7.7 \text { E-07 } \\
1.4 \text { E-11 } \\
3.3 \text { E-11 } \\
\\
8.0 \text { E-13 } \\
2.9 \text { E-11 } \\
3.3 \text { E-10 } \\
1.1 \text { E-09 }\end{array}$ \\
\hline $\begin{array}{l}\text { 296-A-12 } \\
\text { (East Tank Farms) } \\
\text { [WHC] }\end{array}$ & 45.7 & HEPA (2) & $0.0 \mathrm{E}+00$ & 0.0 & - ${ }^{90} \mathrm{Sr}$ & \multicolumn{2}{|c|}{ (did not operate) } \\
\hline $\begin{array}{l}296-\mathrm{A}-17 \\
296-\mathrm{P}-26 \text { (backup) } \\
\text { (East Tank Farms) } \\
\text { [WHC] }\end{array}$ & 15.2 & HEPA (2) & $9.2 \mathrm{E}+07$ & 2.9 & \begin{tabular}{|l}
${ }^{90} \mathrm{Sr}$ \\
${ }^{106} \mathrm{Ru}$ \\
${ }^{113} \mathrm{Sn}$ \\
${ }^{125} \mathrm{Sb}$ \\
${ }^{129} \mathrm{I}$ \\
${ }^{134} \mathrm{Cs}$ \\
$-{ }^{137} \mathrm{Cs}$ \\
${ }^{152} \mathrm{Eu}$ \\
${ }^{154} \mathrm{Eu}$ \\
${ }^{155} \mathrm{Eu}$ \\
${ }^{238} \mathrm{Pu}$ \\
${ }^{239,240} \mathrm{Pu}$ \\
${ }^{241} \mathrm{Am}$
\end{tabular} & $\begin{array}{c}5.4 \mathrm{E}-06 \\
4.0 \mathrm{E}-06 \\
5.3 \mathrm{E}-07 \\
9.5 \mathrm{E}-07 \\
2.7 \mathrm{E}-04 \\
1.8 \mathrm{E}-08 \\
6.2 \mathrm{E}-05 \\
\mathrm{ND} \\
8.8 \mathrm{E}-09 \\
3.1 \mathrm{E}-08 \\
4.6 \mathrm{E}-09 \\
1.4 \mathrm{E}-08 \\
1.2 \mathrm{E}-08\end{array}$ & $\begin{array}{l}1.5 \mathrm{E}-07 \\
1.4 \mathrm{E}-08 \\
1.5 \mathrm{E}-10 \\
6.4 \mathrm{E}-09 \\
5.6 \mathrm{E}-05 \\
4.0 \mathrm{E}-10 \\
3.8 \mathrm{E}-06 \\
\\
4.4 \mathrm{E}-10 \\
6.2 \mathrm{E}-11 \\
7.9 \mathrm{E}-09 \\
2.6 \mathrm{E}-08 \\
3.3 \mathrm{E}-08\end{array}$ \\
\hline $\begin{array}{l}296-A-25 \\
\text { (East Tank Farms) } \\
\text { [WHC] }\end{array}$ & 3.0 & HEPA (2) & $2.6 \mathrm{E}+06$ & 0.1 & $\begin{array}{l}{ }^{90} \mathrm{Sr} \\
{ }^{106} \mathrm{Ru} \\
{ }^{113} \mathrm{Sn} \\
{ }^{125} \mathrm{Sb} \\
{ }^{134} \mathrm{Cs} \\
{ }^{137} \mathrm{Cs} \\
{ }^{152} \mathrm{Eu} \\
{ }^{154} \mathrm{Eu} \\
{ }^{155} \mathrm{Eu} \\
{ }^{238} \mathrm{Pu} \\
{ }^{239} \cdot .20 \mathrm{Pu} \\
{ }^{241} \mathrm{Am}\end{array}$ & $\begin{array}{c}3.2 \mathrm{E}-08 \\
\text { ND } \\
\text { ND } \\
4.0 \mathrm{E}-10 \\
4.0 \mathrm{E}-11 \\
1.4 \mathrm{E}-06 \\
\mathrm{ND} \\
1.4 \mathrm{E}-10 \\
\mathrm{ND} \\
5.7 \mathrm{E}-11 \\
1.7 \mathrm{E}-10 \\
4.1 \mathrm{E}-10\end{array}$ & $\begin{array}{l}8.8 \mathrm{E}-10 \\
2.7 \mathrm{E}-12 \\
9.0 \mathrm{E}-13 \\
8.5 \mathrm{E}-08 \\
7.0 \mathrm{E}-12 \\
9.8 \mathrm{E}-11 \\
3.1 \mathrm{E}-10 \\
1.1 \mathrm{E}-09\end{array}$ \\
\hline
\end{tabular}


Table 2-1

(7 sheets)

\section{Hanford Site Radionuclide Air Emissions Data}

For Major Point Sources.

(major point sources have the potential of $>0.1 \mathrm{mrem} / \mathrm{y}$ EDE to nearest offsite resident)

\begin{tabular}{|c|c|c|c|c|c|c|c|}
\hline $\begin{array}{c}\text { Source } \mathrm{ID}^{\mathbf{b}} \\
\text { (facility/building) } \\
\text { [contractor] }\end{array}$ & $\begin{array}{l}\text { Discharge } \\
\text { Height } \\
\text { (m) }\end{array}$ & $\begin{array}{l}\text { Emission } \\
\text { Control } \\
\text { (stages) }\end{array}$ & $\begin{array}{l}\text { Total } \\
\text { Flow } \\
\left(\mathrm{m}^{3}\right)\end{array}$ & $\begin{array}{c}\text { Annual } \\
\text { Average } \\
\text { Flowrate } \\
\left(\mathrm{m}^{3} / \mathrm{s}\right)\end{array}$ & Radionuclide $^{d}$ & $\begin{array}{l}\text { Annual } \\
\text { Emissions } \\
\text { (Ci) }^{\mathrm{e}}\end{array}$ & $\begin{array}{c}\operatorname{EDE}^{f} \\
\text { for } \\
\text { MEI } \\
\text { (mrem/y) }\end{array}$ \\
\hline $\begin{array}{l}296-\mathrm{A}-40 \\
\text { (East Tank Farms) } \\
{[\mathrm{WHC}]}\end{array}$ & 4.1 & HEPA (2) & $1.4 \mathrm{E}+07$ & 0.4 & $\begin{array}{l}{ }^{90} \mathrm{Sr} \\
{ }^{106} \mathrm{Ru} \\
{ }^{13} \mathrm{Sn} \\
{ }^{123} \mathrm{Sb} \\
{ }^{129} 7 \\
{ }^{134} \mathrm{Cs} \\
{ }^{137} \mathrm{Cs} \\
{ }^{153} \mathrm{Eu} \\
{ }^{154} \mathrm{Eu} \\
{ }^{155} \mathrm{Eu} \\
{ }^{238} \mathrm{Pu} \\
{ }^{239 !} .240 \mathrm{Pu} \\
{ }^{24 !} \mathrm{Am}\end{array}$ & $\begin{array}{c}4.3 \mathrm{E}-09 \\
8.1 \mathrm{E}-07 \\
9.8 \mathrm{E}-08 \\
2.0 \mathrm{E}-07 \\
1.6 \mathrm{E}-05 \\
4.5 \mathrm{E}-10 \\
2.7 \mathrm{E}-08 \\
1.4 \mathrm{E}-09 \\
\mathrm{ND} \\
\mathrm{ND} \\
\mathrm{ND} \\
5.8 \mathrm{E}-11 \\
4.9 \mathrm{E}-10\end{array}$ & 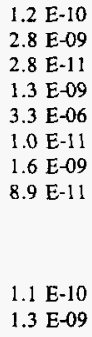 \\
\hline $\begin{array}{l}\text { 296-B-28 } \\
\text { (East Tank Farms) } \\
\text { [WHC] }\end{array}$ & 3.4 & HEPA (2) & $4.2 \mathrm{E}+06$ & 0.1 & $\begin{array}{l}{ }^{90} \mathrm{Sr} \\
{ }^{106} \mathrm{Ru} \\
{ }^{113} \mathrm{Sn} \\
{ }^{125} \mathrm{Sb} \\
{ }^{134} \mathrm{Cs} \\
{ }^{137} \mathrm{Cs} \\
{ }^{152} \mathrm{Eu} \\
{ }^{154} \mathrm{Eu} \\
{ }^{155} \mathrm{Eu} \\
{ }^{238} \mathrm{Pu} \\
{ }^{239,240} \mathrm{Pu} \\
{ }^{24 !} \mathrm{Am}\end{array}$ & $\begin{array}{c}1.2 \mathrm{E}-08 \\
\mathrm{ND} \\
4.2 \mathrm{E}-10 \\
4.8 \mathrm{E}-10 \\
\mathrm{ND} \\
2.7 \mathrm{E}-09 \\
3.1 \mathrm{E}-10 \\
\mathrm{ND} \\
4.2 \mathrm{E}-10 \\
\mathrm{ND} \\
7.1 \mathrm{E}-11 \\
1.3 \mathrm{E}-10\end{array}$ & $\begin{array}{l}3.4 \mathrm{E}-10 \\
1.2 \mathrm{E}-13 \\
3.2 \mathrm{E}-12 \\
1.6 \mathrm{E}-10 \\
2.0 \mathrm{E}-11 \\
8.4 \mathrm{E}-13 \\
1.3 \mathrm{E}-10 \\
3.6 \mathrm{E}-10\end{array}$ \\
\hline $\begin{array}{l}296-\mathrm{C}-5 \\
\text { (East Tank Farms) } \\
\text { [WHC] }\end{array}$ & 14.6 & HEPA (2) & $7.5 \mathrm{E}+07$ & 2.4 & 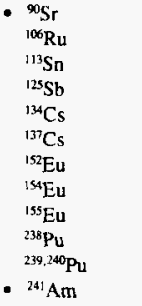 & $\begin{array}{c}2.7 \text { E-07 } \\
\text { ND } \\
1.7 \text { E-09 } \\
6.4 \text { E-09 } \\
4.1 \text { E-09 } \\
2.8 \text { E-07 } \\
\text { ND } \\
3.5 \text { E-11 } \\
3.4 \text { E-09 } \\
4.6 \text { E-10 } \\
3.7 \text { E-09 } \\
2.2 \text { E-09 }\end{array}$ & $\begin{array}{l}7.4 \mathrm{E}-09 \\
4.9 \mathrm{E}-13 \\
4.3 \mathrm{E}-11 \\
9.2 \mathrm{E}-11 \\
1.7 \mathrm{E}-08 \\
1.8 \mathrm{E}-12 \\
6.8 \mathrm{E}-12 \\
7.9 \mathrm{E}-10 \\
6.8 \mathrm{E}-09 \\
6.1 \mathrm{E}-09\end{array}$ \\
\hline
\end{tabular}


Table 2-1

1995 Hanford Site Radionuclide Air Emissions Data

For Major Point Sources.

(major point sources have the potential of $>0.1 \mathrm{mrem} / \mathrm{y}$ EDE to nearest offsite resident)

\begin{tabular}{|c|c|c|c|c|c|c|c|}
\hline $\begin{array}{c}\text { Source } \mathbf{I D}^{\mathrm{b}} \\
\text { (facility/building) } \\
\text { [contractor] }\end{array}$ & $\begin{array}{l}\text { Discharge } \\
\text { Height } \\
\text { (m) }\end{array}$ & $\begin{array}{l}\text { Emission } \\
\text { Control } \\
\text { (stages) }\end{array}$ & $\begin{array}{l}\text { Total } \\
\text { Flow } \\
\left(\mathrm{m}^{3}\right)\end{array}$ & $\begin{array}{c}\text { Annual } \\
\text { Average } \\
\text { Flowrate } \\
\left(\mathrm{m}^{3} / \mathrm{s}\right)\end{array}$ & Radionuclide ${ }^{d}$ & $\begin{array}{c}\text { Annual } \\
\text { Emissions } \\
(\mathrm{Ci})^{\mathbf{e}}\end{array}$ & $\begin{array}{c}\text { EDE }^{f} \\
\text { for } \\
\text { MEI } \\
\text { (mrem/y) }\end{array}$ \\
\hline $\begin{array}{l}\text { 296-P-16 } \\
\text { (East Tank Farms) } \\
\text { [WHC] }\end{array}$ & 4.6 & HEPA (2) & $6.0 \mathrm{E}+07$ & 1.9 & $\begin{array}{l}{ }^{90} \mathrm{Sr} \\
{ }^{106} \mathrm{Ru} \\
{ }^{113} \mathrm{Sn} \\
{ }^{125} \mathrm{Sb} \\
{ }^{134} \mathrm{Cs} \\
{ }^{137} \mathrm{Cs} \\
{ }^{152} \mathrm{Eu} \\
{ }^{154} \mathrm{Eu} \\
{ }_{155}^{15 u} \mathrm{Eu} \\
{ }^{238} \mathrm{Pu} \\
{ }^{239,240} \mathrm{Pu} \\
{ }^{241} \mathrm{Am}\end{array}$ & $\begin{array}{c}4.2 \mathrm{E}-07 \\
\mathrm{ND} \\
\mathrm{ND} \\
3.7 \mathrm{E}-09 \\
\mathrm{ND} \\
1.4 \mathrm{E}-06 \\
1.7 \mathrm{E}-09 \\
\mathrm{ND} \\
4.6 \mathrm{E}-09 \\
1.1 \mathrm{E}-09 \\
1.9 \mathrm{E}-10 \\
6.8 \mathrm{E}-09\end{array}$ & $\begin{array}{l}1.2 \mathrm{E}-08 \\
2.5 \mathrm{E}-11 \\
8.5 \mathrm{E}-08 \\
1.1 \mathrm{E}-10 \\
9.2 \mathrm{E}-12 \\
1.9 \mathrm{E}-09 \\
3.4 \mathrm{E}-10 \\
1.9 \mathrm{E}-08\end{array}$ \\
\hline $\begin{array}{l}296-\mathrm{P}-32 \\
296-\mathrm{P}-33 \\
296-\mathrm{P}-34 \\
\text { (East Tank Farms) } \\
\text { [WHC] }\end{array}$ & 4.6 & HEPA & $1.2 \mathrm{E}+06$ & $<0.1$ & $\begin{array}{l}{ }^{90} \mathrm{Sr} \\
{ }^{133} \mathrm{Sn} \\
{ }^{125} \mathrm{Sb} \\
{ }^{134} \mathrm{Cs} \\
{ }^{137} \mathrm{Cs} \\
{ }^{153} \mathrm{Eu} \\
{ }^{154} \mathrm{Eu} \\
{ }^{155} \mathrm{Eu} \\
{ }^{238} \mathrm{Pu} \\
{ }^{239} / 240 \mathrm{Pu} \\
{ }^{241} \mathrm{Am}\end{array}$ & $\begin{array}{c}3.0 \mathrm{E}-08 \\
4.6 \mathrm{E}-11 \\
1.4 \mathrm{E}-09 \\
1.1 \mathrm{E}-09 \\
2.0 \mathrm{E}-09 \\
3.9 \mathrm{E}-10 \\
4.1 \mathrm{E}-10 \\
2.1 \mathrm{E}-09 \\
\mathrm{ND} \\
3.9 \mathrm{E}-10 \\
8.3 \mathrm{E}-10\end{array}$ & 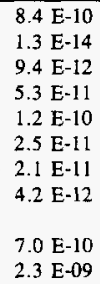 \\
\hline \multicolumn{8}{|c|}{200 West Area Point Sources } \\
\hline $\begin{array}{l}291-Z-1 \\
\text { (PFP) } \\
\text { [WHC] }\end{array}$ & 61.0 & HEPA (1-3) & $3.7 E+09$ & 116.9 & $\begin{array}{l}{ }^{2{ }^{29}} \mathrm{Pu} \\
{ }^{239,240} \mathrm{Pu} \\
{ }^{241} \mathrm{Pu} \\
{ }^{241} \mathrm{Am} \\
\text { total beta }\end{array}$ & $\begin{array}{l}2.3 \mathrm{E}-06 \\
7.6 \mathrm{E}-05 \\
2.0 \mathrm{E}-04 \\
1.5 \mathrm{E}-05 \\
9.5 \mathrm{E}-06\end{array}$ & $\begin{array}{l}2.9 \mathrm{E}-06 \\
1.1 \mathrm{E}-04 \\
4.4 \mathrm{E}-06 \\
3.2 \mathrm{E}-05 \\
2.7 \mathrm{E}-07\end{array}$ \\
\hline $\begin{array}{l}296-\mathrm{S}-15 \\
\text { (West Tank Farms) } \\
\text { [WHC] }\end{array}$ & 4.6 & HEPA (2) & $8.7 \mathrm{E}+07$ & 2.7 & $\begin{array}{l}{ }^{10} \mathrm{Sr} \\
{ }^{106} \mathrm{Ru} \\
{ }^{113} \mathrm{Sn} \\
{ }^{125} \mathrm{Sb} \\
{ }^{134} \mathrm{Cs} \\
{ }^{137} \mathrm{Cs} \\
{ }^{152} \mathrm{Eu} \\
{ }^{154} \mathrm{Eu} \\
{ }^{155} \mathrm{Eu} \\
{ }^{236} \mathrm{Pu} \\
{ }^{239.240} \mathrm{Pu} \\
{ }^{24 i} \mathrm{Am}\end{array}$ & $\begin{array}{c}6.6 \mathrm{E}-08 \\
\mathrm{ND} \\
2.1 \mathrm{E}-09 \\
\mathrm{ND} \\
1.7 \mathrm{E}-09 \\
1.1 \mathrm{E}-07 \\
2.7 \mathrm{E}-09 \\
5.4 \mathrm{E}-09 \\
6.5 \mathrm{E}-09 \\
5.4 \mathrm{E}-10 \\
1.8 \mathrm{E}-09 \\
2.7 \mathrm{E}-09\end{array}$ & $\begin{array}{ll}1.5 & \mathrm{E}-09 \\
4.7 & \mathrm{E}-13 \\
2.9 & \mathrm{E}-11 \\
5.1 & \mathrm{E}-09 \\
1.3 & \mathrm{E}-10 \\
2.1 & \mathrm{E}-10 \\
1.0 & \mathrm{E}-11 \\
6.9 & \mathrm{E}-10 \\
2.5 & \mathrm{E}-09 \\
5.7 & \mathrm{E}-09\end{array}$ \\
\hline
\end{tabular}


Table 2-1

\begin{tabular}{|c|c|c|c|c|c|c|c|}
\hline \multicolumn{8}{|c|}{$\begin{array}{l}1995 \text { Hanford Site Radionuclide Air Emissions Data } \\
\text { For Major Point Sources. } \\
\text { nt sources have the potential of }>0.1 \text { mrem/y EDE to nearest offsite resident) }\end{array}$} \\
\hline $\begin{array}{c}\begin{array}{c}\text { Source } \text { ID }^{\mathbf{b}} \\
\text { (facility/building) } \\
\text { [contractor] }\end{array} \\
\end{array}$ & $\begin{array}{c}\text { Discharge } \\
\text { Height } \\
\text { (m) }\end{array}$ & $\begin{array}{l}\text { Emission } \\
\text { Controlf } \\
\text { (stages) }\end{array}$ & $\begin{array}{l}\text { Total } \\
\text { Flow } \\
\left(\mathrm{m}^{3}\right)\end{array}$ & $\begin{array}{c}\text { Annual } \\
\text { Average } \\
\text { Flowrate }^{\mathrm{i}} \\
\left(\mathbf{m}^{3} / \mathbf{s}\right)\end{array}$ & Radionuclide $^{\mathbf{d}}$ & $\begin{array}{c}\text { Annual } \\
\text { Emissions } \\
\text { (Ci) }^{*}\end{array}$ & $\begin{array}{c}\text { EDE }^{f} \\
\text { for } \\
\text { MEI } \\
(\mathrm{mrem} / \mathrm{y})\end{array}$ \\
\hline $\begin{array}{l}296-S-22 \\
\text { (West Tank Farms) } \\
\text { [WHC] }\end{array}$ & 3.7 & HEPA (2) & $1.7 \mathrm{E}+06$ & 0.1 & 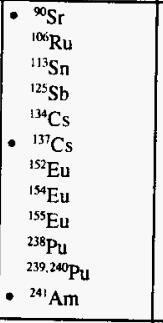 & $\begin{array}{c}2.1 \mathrm{E}-08 \\
\mathrm{ND} \\
\mathrm{ND} \\
3.2 \mathrm{E}-10 \\
\mathrm{ND} \\
4.2 \mathrm{E}-10 \\
\mathrm{ND} \\
\mathrm{ND} \\
5.0 \mathrm{E}-10 \\
\mathrm{ND} \\
1.4 \mathrm{E}-10 \\
4.1 \mathrm{E}-10\end{array}$ & $\begin{array}{l}4.7 \mathrm{E}-10 \\
1.7 \mathrm{E}-12 \\
1.9 \mathrm{E}-11 \\
7.9 \mathrm{E}-13 \\
2.0 \mathrm{E}-10 \\
8.7 \mathrm{E}-10\end{array}$ \\
\hline $\begin{array}{l}\text { 296-T-18 } \\
\text { (West Tank Farms) } \\
\text { [WHCl }\end{array}$ & 3.7 & HEPA (2) & $5.4 \mathrm{E}+06$ & 0.2 & \begin{tabular}{|l}
${ }^{90} \mathrm{Sr}$ \\
${ }^{106} \mathrm{Ru}$ \\
${ }^{113} \mathrm{Sn}$ \\
${ }^{125} \mathrm{Sb}$ \\
${ }^{134} \mathrm{Cs}$ \\
$\cdot{ }^{137} \mathrm{Cs}$ \\
${ }^{152} \mathrm{Eu}$ \\
${ }^{754} \mathrm{Eu}$ \\
${ }^{155} \mathrm{Eu}$ \\
${ }^{238} \mathrm{Pu}$ \\
- ${ }^{239.240} \mathrm{Pu}$ \\
\end{tabular} & $\begin{array}{c}1.0 \mathrm{E}-10 \\
\mathrm{ND} \\
\mathrm{ND} \\
\mathrm{ND} \\
2.3 \mathrm{E}-10 \\
2.6 \mathrm{E}-10 \\
\mathrm{ND} \\
\mathrm{ND} \\
6.2 \mathrm{E}-10 \\
2.1 \mathrm{E}-11 \\
1.0 \mathrm{E}-09 \\
5.8 \mathrm{E}-10 \\
\end{array}$ & $\begin{array}{l}2.2 \mathrm{E}-12 \\
4.0 \mathrm{E}-12 \\
1.2 \mathrm{E}-11 \\
\\
9.8 \mathrm{E}-13 \\
2.7 \mathrm{E}-11 \\
1.4 \mathrm{E}-09 \\
1.2 \mathrm{E}-09 \\
\end{array}$ \\
\hline \multicolumn{8}{|c|}{300 Area Point Sources } \\
\hline $\begin{array}{l}340-N T-E X \\
\text { (340 Waste Handling) } \\
\text { [WHC] }\end{array}$ & 5.5 & $\begin{array}{l}\text { HEPA (2), } \\
\text { charcoal }\end{array}$ & $2.9 \mathrm{E}+07$ & 0.9 & \begin{tabular}{|l}
${ }^{90} \mathrm{Sr}$ \\
${ }^{129} \mathrm{~T}$ \\
${ }^{137} \mathrm{Cs}$ \\
. \\
${ }^{239.240} \mathrm{Pu}$ \\
total alpha \\
total beta \\
\end{tabular} & $\begin{array}{c}\text { ND } \\
\text { ND } \\
\text { ND } \\
\text { ND } \\
6.7 \mathrm{E}-08 \\
1.3 \mathrm{E}-07\end{array}$ & $\begin{array}{l}5.4 \mathrm{E}-06 \\
1.4 \mathrm{E}-07\end{array}$ \\
\hline $\begin{array}{l}\text { EP-324-01-S } \\
\text { (324 Bldg.) } \\
{[\text { PNNL] }}\end{array}$ & 48.4 & HEPA & $1.1 \mathrm{E}+09$ & 34.9 & \begin{tabular}{|l|}
${ }^{3} \mathrm{H}$ (as HTO) \\
${ }^{3} \mathrm{H}$ (as HT) \\
${ }^{60} \mathrm{Co}$ \\
- ${ }^{80} \mathrm{Sr}$ \\
${ }^{134} \mathrm{Cs}$ \\
- ${ }^{137} \mathrm{Cs}$ \\
${ }^{154} \mathrm{Eu}$ \\
${ }^{155} \mathrm{Eu}$ \\
${ }^{238} \mathrm{Pu}$ \\
${ }^{239} / 240 \mathrm{Pu}$ \\
${ }^{241} \mathrm{Am}$ \\
unsp. alpha \\
unsp. beta \\
\end{tabular} & $\begin{array}{c}1.4 \mathrm{E}-01 \\
3.2 \mathrm{E}+00 \\
\mathrm{ND} \\
2.1 \mathrm{E}-08 \\
\mathrm{ND} \\
\mathrm{ND} \\
\mathrm{ND} \\
\mathrm{ND} \\
4.2 \mathrm{E}-10 \\
1.3 \mathrm{E}-09 \\
3.4 \mathrm{E}-09 \\
9.5 \mathrm{E}-08 \\
3.7 \mathrm{E}-07\end{array}$ & $\begin{array}{ll}3.2 & \mathrm{E}-08 \\
1.1 & \mathrm{E}-07 \\
4.3 \mathrm{E}-07 \\
7.7 \mathrm{E}-06 \\
3.9 \mathrm{E}-07\end{array}$ \\
\hline
\end{tabular}


Table 2-1

\section{Hanford Site Radionuclide Air Emissions Data}

For Major Point Sources.

(major point sources have the potential of $>0.1 \mathrm{mrem} / \mathrm{y}$ EDE to nearest offsite resident)

\begin{tabular}{|c|c|c|c|c|c|c|c|}
\hline $\begin{array}{c}\text { Source ID } \\
\text { (facility/building) } \\
\text { [contractor] }\end{array}$ & $\begin{array}{l}\text { Discharge } \\
\text { Height } \\
\text { (m) }\end{array}$ & $\begin{array}{l}\text { Emission } \\
\text { Control }^{e} \\
\text { (stages) }\end{array}$ & $\begin{array}{l}\text { Total } \\
\text { Flow } \\
\left(\mathrm{m}^{3}\right)\end{array}$ & $\begin{array}{c}\text { Annual } \\
\text { Average } \\
\text { Flowrate } \\
\left(\mathbf{m}^{3} / \mathbf{s}\right)\end{array}$ & Radionuclide $^{d}$ & $\begin{array}{l}\text { Annual } \\
\text { Emissions } \\
\text { (Ci) }^{e}\end{array}$ & $\begin{array}{c}\text { EDE }^{I} \\
\text { for } \\
\text { MEI } \\
\text { (mrem/y) }\end{array}$ \\
\hline $\begin{array}{l}\text { EP-325-01-S } \\
\text { (325 Bldg.) } \\
\text { [PNNL] }\end{array}$ & 17.9 & HEPA & $2.0 \mathrm{E}+09$ & 63.4 & 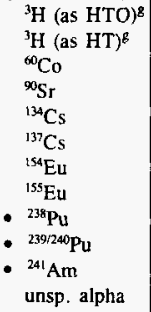 & $\begin{array}{c}2.6 \mathrm{E}+00 \\
5.1 \mathrm{E}-01 \\
\mathrm{ND} \\
\mathrm{ND} \\
\mathrm{ND} \\
\mathrm{ND} \\
\mathrm{ND} \\
\mathrm{ND} \\
\mathrm{ND} \\
3.6 \mathrm{E}-10 \\
1.2 \mathrm{E}-09 \\
1.5 \mathrm{E}-07\end{array}$ & $\begin{array}{l}2.2 \text { E-04 } \\
4.3 \text { E-05 } \\
\\
2.9 \text { E-08 } \\
1.5 \text { E-07 }\end{array}$ \\
\hline $\begin{array}{l}\text { EP-327-0l-S } \\
\text { (327 Bldg.) } \\
\text { [PNNL] }\end{array}$ & 13.7 & HEPA & $7.2 E+08$ & 22.8 & $\begin{array}{l}{ }^{3} \mathrm{H} \text { (as HTO) } \\
{ }^{3} \mathrm{H} \text { (as HT) } \\
{ }^{60} \mathrm{Co} \\
{ }^{90} \mathrm{Sr} \\
{ }^{134} \mathrm{Cs} \\
{ }^{137} \mathrm{Cs} \\
{ }^{154} \mathrm{Eu} \\
{ }^{155} \mathrm{Eu} \\
{ }^{220} \mathrm{Rn}^{n} \\
{ }^{222} \mathrm{Rn}^{6} \\
{ }^{238} \mathrm{Pu} \\
{ }^{239.240} \mathrm{Pu} \\
{ }^{241} \mathrm{Am} \\
\text { unsp. alpha } \\
\text { unsp. beta }\end{array}$ & $\begin{array}{c}5.9 \mathrm{E}-02 \\
1.3 \mathrm{E}-01 \\
\mathrm{ND} \\
2.1 \mathrm{E}-07 \\
\mathrm{ND} \\
1.5 \mathrm{E}-06 \\
\mathrm{ND} \\
\mathrm{ND} \\
7.9 \mathrm{E}+01 \\
4.0 \mathrm{E}-01 \\
2.1 \mathrm{E}-09 \\
1.2 \mathrm{E}-08 \\
6.0 \mathrm{E}-09 \\
1.9 \mathrm{E}-07 \\
2.2 \mathrm{E}-06\end{array}$ & $\begin{array}{l}5.0 \mathrm{E}-06 \\
1.1 \mathrm{E}-05 \\
2.2 \mathrm{E}-07 \\
3.2 \mathrm{E}-06 \\
\\
3.3 \mathrm{E}-03 \\
1.9 \mathrm{E}-04 \\
1.6 \mathrm{E}-07 \\
9.7 \mathrm{E}-07 \\
7.6 \mathrm{E}-07 \\
1.5 \mathrm{E}-05 \\
2.3 \mathrm{E}-06\end{array}$ \\
\hline $\begin{array}{l}\text { EP-327-02-V } \\
\text { (327 Decon. Cell) } \\
{[\text { PNNL] }}\end{array}$ & 9.1 & HEPA & $1.2 \mathrm{E}+07$ & 0.4 & $\begin{array}{l}{ }^{60} \mathrm{Co} \\
{ }^{90} \mathrm{Sr} \\
{ }^{134} \mathrm{Cs} \\
{ }^{137} \mathrm{Cs} \\
{ }^{154} \mathrm{Eu} \\
{ }^{155} \mathrm{Eu} \\
{ }^{238} \mathrm{Pu} \\
{ }^{239,240} \mathrm{Pu} \\
{ }^{241} \mathrm{Am} \\
\text { unsp. alpha } \\
\text { unsp. beta }\end{array}$ & $\begin{array}{c}\text { ND } \\
1.8 \mathrm{E}-10 \\
1.2 \mathrm{E}-09 \\
\mathrm{ND} \\
\mathrm{ND} \\
1.0 \mathrm{E}-09 \\
1.1 \mathrm{E}-11 \\
3.3 \mathrm{E}-11 \\
3.8 \mathrm{E}-11 \\
9.0 \mathrm{E}-10 \\
2.0 \mathrm{E}-09\end{array}$ & $\begin{array}{l}1.9 \text { E-10 } \\
9.8 \text { E-10 } \\
3.5 \text { E-11 } \\
8.4 \text { E-10 } \\
2.7 \text { E-09 } \\
4.8 \text { E-09 } \\
7.3 \text { E-08 } \\
2.1 \text { E-09 }\end{array}$ \\
\hline
\end{tabular}


Table 2-1

\section{Hanford Site Radionuclide Air Emissions Data}

For Major Point Sources.

(major point sources have the potential of $>0.1 \mathrm{mrem} / \mathrm{y}$ EDE to nearest offsite resident) ${ }^{\mathrm{a}}$

\begin{tabular}{|c|c|c|c|c|c|c|c|}
\hline $\begin{array}{c}\text { Source ID } \\
\text { (facility/building) } \\
\text { [contractor] }\end{array}$ & $\begin{array}{l}\text { Discharge } \\
\text { Height } \\
\text { (m) }\end{array}$ & $\begin{array}{l}\text { Emission } \\
\text { Control } \\
\text { (stages) }\end{array}$ & $\begin{array}{l}\text { Total } \\
\text { Flow } \\
\left(\mathrm{m}^{3}\right)\end{array}$ & $\begin{array}{c}\text { Annual } \\
\text { Average } \\
\text { Flowrate } \\
\left(\mathbf{m}^{3} / \mathbf{s}\right)\end{array}$ & Radionuclide $^{d}$ & $\begin{array}{c}\text { Annual } \\
\text { Emissions } \\
\text { (Ci) }^{\mathbf{e}}\end{array}$ & $\begin{array}{c}\operatorname{EDE}^{I} \\
\text { for } \\
\text { MEI } \\
(\mathbf{m r e m} / \mathbf{y})\end{array}$ \\
\hline $\begin{array}{l}\text { EP-3720-01-S } \\
\text { (3720 Bldg.) } \\
\text { [PNNL] }\end{array}$ & 9.1 & HEPA & $3.3 E+08$ & 10.5 & $\begin{array}{l}{ }^{60} \mathrm{Co} \\
{ }^{90} \mathrm{Sr} \\
{ }^{134} \mathrm{Cs} \\
{ }^{137} \mathrm{Cs} \\
{ }^{154} \mathrm{Eu} \\
{ }^{155} \mathrm{Eu} \\
{ }^{238} \mathrm{Pu} \\
{ }^{239 / 240} \mathrm{Pu} \\
\text { - }{ }^{241} \mathrm{Am} \\
\text { unsp. alpha }\end{array}$ & $\begin{array}{c}\text { ND } \\
\text { ND } \\
2.6 \mathrm{E}-08 \\
4.4 \mathrm{E}-08 \\
\mathrm{ND} \\
4.2 \mathrm{E}-08 \\
\mathrm{ND} \\
6.4 \mathrm{E}-10 \\
\mathrm{ND} \\
2.9 \mathrm{E}-08\end{array}$ & $\begin{array}{l}2.1 \mathrm{E}-08 \\
9.4 \mathrm{E}-08 \\
1.5 \mathrm{E}-09 \\
5.2 \mathrm{E}-08 \\
2.3 \mathrm{E}-06\end{array}$ \\
\hline
\end{tabular}

Notes:

a Determining the state of National Emission Standards for Hazardous Air Pollutants (NESHAP) Subpart H compliance for each point source involved using nearest offsite residences, which differed from the MEI.

b ID = Identification, i.e., the alpha-numeric designator for the respective point source; WHC = Westinghouse Hanford Company; PNNL = Pacific Northwest National Laboratory (Battelle); BHI = Bechtel Hanford. Inc.

c Efficiencies are: $\geq 99.95 \%$ for HEPA; $\geq 95 \%$ for charcoal; $\geq 99.8 \%$ for sand filter; $0 \%$ for no emission control.

d Bullets, "•", identify specific radionuclide sampling and analysis required by 40 CFR 61 Subpart H. "unsp. alpha" and "unsp. beta" stands for unspecified alpha and beta, respectively. Unspecified alpha releases are the result of subtracting the activity released for alpha emitters specifically analyzed for from the total activity released calculated from the total alpha analyses. The same process was used to calculate releases for unspecified beta emitters.

e 1 Curie $=3.7 \mathrm{E}+10$ becquerel; $\mathrm{ND}=$ not detected (i.e. either the radionuclide was not detected in any sample during the year, or the average of all the measurements for that given radionuclide or type of radioactivity made during the year was below background levels).

f $\mathrm{EDE}=$ Effective dose equivalent for the MEI (at Sagemore Road farm east of the 300 Area), not the nearest residence; $1 \mathrm{mrem}=1 \mathrm{E}-02 \mathrm{mSv}$.

g HTO is tritium as condensable water vapor; $\mathrm{HT}$ is tritium as incondensable gas.

h A description of the offsite dose effects of radon emissions is provided in Section 5.3 .

i The annual average flowrate is calculated by dividing the total flow (discharge volume) by the number of seconds in 365 days. 
DOE/RL-96-37

This page intentionally left blank. 
Table 2-2

(11 sheets)

1995 Hanford Site Radionuclide Air Emissions Data

For Minor Point Sources.

(minor point sources have the potential of $<0.1 \mathrm{mren} / \mathrm{y}$ EDE to nearest offsite resident)

\begin{tabular}{|c|c|c|c|c|c|c|}
\hline $\begin{array}{c}\text { Source ID } \\
\text { (facility/building) } \\
\text { [contractor] }\end{array}$ & $\begin{array}{c}\text { Discharge } \\
\text { Height } \\
\text { (m) }\end{array}$ & $\begin{array}{l}\text { Emission } \\
\text { Control }^{\text {b }}\end{array}$ & $\begin{array}{l}\text { Total } \\
\text { Flow } \\
\left(\mathbf{m}^{3}\right)\end{array}$ & $\begin{array}{c}\text { Annual } \\
\text { Average } \\
\text { Flowrate } \\
\left(\mathrm{m}^{3} / \mathbf{s}\right)\end{array}$ & Radionuclide & $\begin{array}{c}\text { Annual } \\
\text { Emissions } \\
\text { (Ci) }^{\mathrm{c}}\end{array}$ \\
\hline \multicolumn{7}{|c|}{100 Area Point Sources } \\
\hline $\begin{array}{l}116-\mathrm{N} \\
(100 \mathrm{~N} \text { Area }) \\
{[\mathrm{BHI}]}\end{array}$ & 61.3 & $\begin{array}{l}\text { HEPA, } \\
\text { charcoal }\end{array}$ & $1.6 \mathrm{E}+09$ & 51.9 & $\begin{array}{l}{ }^{60} \mathrm{Co} \\
{ }^{90} \mathrm{Sr} \\
{ }^{137} \mathrm{Cs} \\
{ }^{238} \mathrm{Pu} \\
{ }^{2391240} \mathrm{Pu} \\
{ }^{241} \mathrm{Am}\end{array}$ & $\begin{array}{c}\text { 6.6 E-06 } \\
\text { ND } \\
\text { ND } \\
\text { ND } \\
5.8 \mathrm{E}-08 \\
4.2 \mathrm{E}-08\end{array}$ \\
\hline $\begin{array}{l}105-\mathrm{N} 14 \mathrm{ft} \\
(100 \mathrm{~N} \text { Area }) \\
{[\mathrm{BHI}]}\end{array}$ & 8.5 & HEPA & $9.5 E+07$ & 3.0 & $\begin{array}{l}{ }^{60} \mathrm{Co} \\
{ }^{90} \mathrm{Sr} \\
{ }^{125} \mathrm{Sb} \\
{ }^{238} \mathrm{Pu} \\
{ }^{239 / 240} \mathrm{Pu} \\
{ }^{291} \mathrm{Am}\end{array}$ & $\begin{array}{c}\mathrm{ND} \\
4.4 \mathrm{E}-07 \\
2.7 \mathrm{E}-07 \\
\text { ND } \\
2.5 \mathrm{E}-09 \\
1.6 \mathrm{E}-08\end{array}$ \\
\hline $\begin{array}{l}107-\mathrm{N} \\
(100 \mathrm{~N} \text { Area }) \\
{[\mathrm{BHI}]}\end{array}$ & 12.0 & HEPA & $1.1 \mathrm{E}+08$ & 3.4 & $\begin{array}{l}{ }^{60} \mathrm{Co} \\
{ }^{90} \mathrm{Sr} \\
{ }^{155} \mathrm{Eu} \\
{ }^{238} \mathrm{Pu} \\
{ }^{239} / 240 \mathrm{Pu} \\
{ }^{241} \mathrm{Am}\end{array}$ & $\begin{array}{c}1.0 \mathrm{E}-07 \\
1.5 \mathrm{E}-07 \\
2.0 \mathrm{E}-07 \\
\mathrm{ND} \\
2.3 \mathrm{E}-09 \\
3.6 \mathrm{E}-09\end{array}$ \\
\hline $\begin{array}{l}09-\mathrm{N}-\mathrm{Z}-1 \\
(100 \mathrm{~N} \text { Area }) \\
{[\mathrm{BHI}]}\end{array}$ & 13.7 & none & $0.0 \mathrm{E}+00$ & 0.0 & \multicolumn{2}{|c|}{ (did not operate) } \\
\hline $\begin{array}{l}109-\mathrm{N}-\mathrm{C}-6 \\
(100 \mathrm{~N} \text { Area) } \\
\text { [BHI) }\end{array}$ & 13.7 & none & $0.0 \mathrm{E}+00$ & 0.0 & \multicolumn{2}{|c|}{ (did not operate) } \\
\hline $\begin{array}{l}\text { 105-KE Basin } \\
\text { (100 K Area) } \\
\text { [WHC] }\end{array}$ & 12.8 & none & $5.3 \mathrm{E}+08$ & 16.9 & 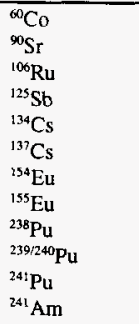 & $\begin{array}{ll}1.9 & \mathrm{E}-06 \\
5.9 & \mathrm{E}-06 \\
1.1 & \mathrm{E}-05 \\
2.5 & \mathrm{E}-06 \\
4.6 & \mathrm{E}-07 \\
2.4 & \mathrm{E}-04 \\
5.8 & \mathrm{E}-06 \\
1.2 & \mathrm{E}-06 \\
2.3 & \mathrm{E}-06 \\
1.5 & \mathrm{E}-05 \\
2.1 & \mathrm{E}-04 \\
5.6 & \mathrm{E}-06\end{array}$ \\
\hline
\end{tabular}


Table 2-2

(11 sheets)

\begin{tabular}{|c|c|c|c|c|c|c|}
\hline \multicolumn{7}{|c|}{$\begin{array}{l}1995 \text { Hanford Site Radionuclide Air Emissions Data } \\
\text { For Minor Point Sources. } \\
\text { (minor point sources have the potential of }<0.1 \text { mrem } / y \text { EDE to nearest offsite resident) }\end{array}$} \\
\hline $\begin{array}{l}\text { Source } \text { ID }^{\mathfrak{a}} \\
\text { (facility/building) } \\
\text { [contractor] }\end{array}$ & $\begin{array}{c}\text { Discharge } \\
\text { Height } \\
\text { (m) }\end{array}$ & $\begin{array}{l}\text { Emission } \\
\text { Control }^{b}\end{array}$ & $\begin{array}{l}\text { Total } \\
\text { Flow } \\
\left(\mathbf{m}^{3}\right)\end{array}$ & $\begin{array}{l}\text { Annual } \\
\text { Average } \\
\text { Flowrate }^{\mathrm{e}} \\
\left(\mathbf{m}^{3} / \mathbf{s}\right)\end{array}$ & Radionuclide & $\begin{array}{c}\text { Annual } \\
\text { Emissions } \\
\text { (Ci) }^{\mathbf{c}}\end{array}$ \\
\hline $\begin{array}{l}\text { 105-KW Basin } \\
\text { (100 K Area) } \\
{[\text { WHC] }}\end{array}$ & 12.8 & none & $5.0 \mathrm{E}+08$ & 16.0 & $\begin{array}{l}{ }^{60} \mathrm{Co} \\
{ }^{90} \mathrm{Sr} \\
{ }^{134} \mathrm{Cs} \\
{ }^{137} \mathrm{Cs} \\
{ }^{154} \mathrm{Eu} \\
{ }^{155} \mathrm{Eu} \\
{ }^{238} \mathrm{Pu} \\
{ }^{239 / 24} \mathrm{Pu} \\
{ }^{241} \mathrm{Pu} \\
{ }^{241} \mathrm{Am}\end{array}$ & $\begin{array}{l}4.7 \mathrm{E}-07 \\
3.8 \mathrm{E}-07 \\
8.6 \mathrm{E}-07 \\
9.9 \mathrm{E}-06 \\
1.9 \mathrm{E}-06 \\
7.8 \mathrm{E}-09 \\
3.4 \mathrm{E}-09 \\
2.0 \mathrm{E}-08 \\
4.4 \mathrm{E}-07 \\
1.5 \mathrm{E}-08\end{array}$ \\
\hline $\begin{array}{l}1706-\mathrm{KER}-27 \mathrm{ft} \\
\text { (100 K Area) } \\
\text { [WHC] }\end{array}$ & 0.9 & HEPA & $3.7 \mathrm{E}+07$ & 1.2 & $\begin{array}{l}{ }^{60} \mathrm{Co} \\
{ }^{90} \mathrm{Sr} \\
{ }^{134} \mathrm{Cs} \\
{ }^{137} \mathrm{Cs} \\
{ }^{238} \mathrm{Pu} \\
{ }^{239 i 240} \mathrm{Pu} \\
{ }^{241} \mathrm{Am}\end{array}$ & $\begin{array}{c}\text { ND } \\
6.0 \text { E-09 } \\
1.2 \text { E-08 } \\
\text { ND } \\
9.1 \mathrm{E}-11 \\
6.5 \mathrm{E}-10 \\
6.9 \mathrm{E}-10\end{array}$ \\
\hline $\begin{array}{l}\text { 1706-KE } \\
\text { (100 K Aгea) } \\
\text { [WHC] }\end{array}$ & 7.6 & HEPA & $1.8 \mathrm{E}+08$ & 5.7 & $\begin{array}{l}{ }^{60} \mathrm{Co} \\
{ }^{90} \mathrm{Sr} \\
{ }^{137} \mathrm{Cs} \\
{ }^{154} \mathrm{Eu} \\
{ }^{155} \mathrm{Eu} \\
{ }^{239 / 240} \mathrm{Pu} \\
{ }^{241} \mathrm{Am}\end{array}$ & $\begin{array}{c}3.3 \mathrm{E}-08 \\
\mathrm{ND} \\
\mathrm{ND} \\
6.0 \mathrm{E}-07 \\
1.3 \mathrm{E}-07 \\
3.6 \mathrm{E}-09 \\
9.0 \mathrm{E}-09\end{array}$ \\
\hline \multicolumn{7}{|c|}{200 East Area Point Sources } \\
\hline $\begin{array}{l}\text { 296-A-2 } \\
\text { (PUREX) } \\
{[\text { WHC] }}\end{array}$ & 20.7 & HEPA & $3.3 \mathrm{E}+07$ & 1.0 & $\begin{array}{l}\text { total alpha } \\
\text { total beta }\end{array}$ & $\begin{array}{l}3.3 \mathrm{E}-09 \\
5.8 \mathrm{E}-09\end{array}$ \\
\hline $\begin{array}{l}296-\mathrm{A}-3 \\
\text { (PUREX) } \\
{[\mathrm{WHC}]}\end{array}$ & 24.1 & $\overline{\text { HEPA }}$ & $4.4 \mathrm{E}+07$ & 1.4 & $\begin{array}{l}\text { total alpha } \\
\text { total beta }\end{array}$ & $\begin{array}{l}5.2 \mathrm{E}-09 \\
1.1 \mathrm{E}-07\end{array}$ \\
\hline $\begin{array}{l}296-A-5 A \\
296-A-5 B \\
\text { (PUREX) } \\
{[\text { WHC] }}\end{array}$ & 17.1 & HEPA & $2.8 \mathrm{E}+08$ & 8.9 & $\begin{array}{l}\text { total alpha } \\
\text { total beta }\end{array}$ & $\begin{array}{l}5.4 \mathrm{E}-08 \\
8.8 \mathrm{E}-08\end{array}$ \\
\hline $\begin{array}{l}296-A-6 \\
\text { (PUREX) } \\
\text { [WHC] }\end{array}$ & 20.7 & HEPA & $1.8 \mathrm{E}+08$ & 5.7 & $\begin{array}{l}\text { total alpha } \\
\text { total beta }\end{array}$ & $\begin{array}{c}\text { ND } \\
1.4 \mathrm{E}-08\end{array}$ \\
\hline $\begin{array}{l}296-A-7 \\
\text { (PUREX) } \\
\text { [WHC] }\end{array}$ & 20.7 & $\overline{\text { HEPA }}$ & $2.7 \mathrm{E}+08$ & 8.5 & $\begin{array}{l}\text { total alpha } \\
\text { total beta }\end{array}$ & $\begin{array}{r}1.2 \mathrm{E}-08 \\
-2.1 \mathrm{E}-07\end{array}$ \\
\hline
\end{tabular}


Table 2-2

(11 sheets)

\begin{tabular}{|c|c|c|c|c|c|c|}
\hline \multicolumn{7}{|c|}{$\begin{array}{l}1995 \text { Hanford Site Radionuclide Air Emissions Data } \\
\text { For Minor Point Sources. } \\
\text { (minor point sources have the potential of }<0.1 \mathrm{mrem} / \mathrm{y} \text { EDE to nearest offsite resident) }\end{array}$} \\
\hline $\begin{array}{l}\text { Source } \mathrm{ID}^{\mathrm{a}} \\
\text { (facility/building) } \\
\text { [contractor] }\end{array}$ & $\begin{array}{c}\text { Discharge } \\
\text { Height } \\
\text { (m) }\end{array}$ & $\begin{array}{l}\text { Emission } \\
\text { Control }^{b}\end{array}$ & $\begin{array}{l}\text { Total } \\
\text { Flow } \\
\left(\mathbf{m}^{3}\right)\end{array}$ & $\begin{array}{c}\text { Annual } \\
\text { Average } \\
\text { Flowrate } \\
\left(\mathbf{m}^{3} / \mathbf{s}\right)\end{array}$ & Radionuclide & $\begin{array}{c}\text { Annual } \\
\text { Emissions } \\
\text { (Ci) }^{\mathrm{c}}\end{array}$ \\
\hline $\begin{array}{l}\text { 296-A-8 } \\
\text { (PUREX) } \\
\text { [WHC] }\end{array}$ & 20.7 & HEPA & $2.1 \mathrm{E}+08$ & 6.6 & $\begin{array}{l}\text { total alpha } \\
\text { total beta }\end{array}$ & $\begin{array}{l}7.1 \text { E-08 } \\
5.7 \text { E-07 }\end{array}$ \\
\hline $\begin{array}{l}296-\mathrm{A}-10 \\
\text { (PUREX) } \\
\text { [WHC] }\end{array}$ & 4.6 & HEPA & $1.0 \mathrm{E}+08$ & 3.2 & $\begin{array}{l}\text { total alpha } \\
\text { total beta }\end{array}$ & $\begin{array}{l}\text { ND } \\
\text { ND }\end{array}$ \\
\hline $\begin{array}{l}296-\mathrm{A}-14 \\
\text { (PUREX) } \\
{[\text { WHC] }}\end{array}$ & 9.1 & HEPA & $6.9 \mathrm{E}+07$ & 2.2 & $\begin{array}{l}\text { total alpha } \\
\text { total beta }\end{array}$ & $\begin{array}{l}1.3 \mathrm{E}-08 \\
2.2 \mathrm{E}-08\end{array}$ \\
\hline $\begin{array}{l}296-\mathrm{A}-24 \\
\text { (PUREX) } \\
{[\text { WHC] }}\end{array}$ & 24.0 & None & $0.0 \mathrm{E}+00$ & 0.0 & \multicolumn{2}{|c|}{ (did not operate) } \\
\hline $\begin{array}{l}\text { 296-B-5 } \\
\text { (B Plant) } \\
{[\text { WHC] }}\end{array}$ & 3.7 & HEPA & $2.2 \mathrm{E}+07$ & 0.7 & $\begin{array}{l}\text { total alpha } \\
\text { total beta }\end{array}$ & $\begin{array}{l}4.5 \mathrm{E}-09 \\
1.6 \mathrm{E}-08\end{array}$ \\
\hline $\begin{array}{l}296-B-10 \\
\text { (B Plant) } \\
\text { [WHC] }\end{array}$ & 22.9 & HEPA & $3.0 \mathrm{E}+08$ & 9.4 & $\begin{array}{l}{ }^{90} \mathrm{Sr} \\
{ }^{106} \mathrm{Ru} \\
{ }^{129} \mathrm{Sb} \\
{ }^{137} \mathrm{Cs} \\
{ }^{152} \mathrm{Eu} \\
{ }^{154} \mathrm{Eu} \\
{ }^{155} \mathrm{Eu} \\
{ }^{238} \mathrm{Pu} \\
{ }^{239 i 240} \mathrm{Pu} \\
{ }^{241} \mathrm{Am} \\
\end{array}$ & $\begin{array}{c}6.0 \mathrm{E}-06 \\
2.2 \mathrm{E}-09 \\
2.2 \mathrm{E}-08 \\
2.9 \mathrm{E}-06 \\
1.6 \mathrm{E}-08 \\
5.8 \mathrm{E}-08 \\
1.1 \mathrm{E}-08 \\
\mathrm{ND} \\
5.2 \mathrm{E}-10 \\
1.3 \mathrm{E}-08\end{array}$ \\
\hline $\begin{array}{l}\text { 296-B-13 } \\
\text { (B Plant) } \\
\text { [WHC] }\end{array}$ & 3.5 & HEPA & $1.0 \mathrm{E}+07$ & 0.3 & $\begin{array}{l}\text { total aipha } \\
\text { total beta }\end{array}$ & $\begin{array}{l}2.8 \mathrm{E}-09 \\
1.5 \mathrm{E}-08\end{array}$ \\
\hline $\begin{array}{l}296-\mathrm{A}-13 \\
\text { (East Tank Farms) } \\
{[\text { WHC] }}\end{array}$ & 38.1 & HEPA & $0.0 \mathrm{E}+00$ & 0.0 & \multicolumn{2}{|c|}{ (did not operate) } \\
\hline $\begin{array}{l}296-\mathrm{A}-18 \\
\text { (East Tank Farms) } \\
{[\text { WHC] }}\end{array}$ & 4.6 & $\overline{\mathrm{HEPA}}$ & $0.0 \mathrm{E}+00$ & $\overline{0.0}$ & \multicolumn{2}{|c|}{ (did not operate) } \\
\hline $\begin{array}{l}296-A-19 \\
\text { (East Tank Farms) } \\
\text { [WHC] }\end{array}$ & 4.6 & HEPA & $0.0 \mathrm{E}+00$ & 0.0 & \multicolumn{2}{|c|}{ (did not operate) } \\
\hline $\begin{array}{l}296-\mathrm{A}-20 \\
\text { (East Tank Farms) } \\
\text { [WHC] }\end{array}$ & 7.3 & HEPA & $1.6 \mathrm{E}+07$ & 0.5 & $\begin{array}{l}\text { total alpha } \\
\text { total beta }\end{array}$ & $\begin{array}{l}1.1 \text { E-08 } \\
3.1 \text { E-08 }\end{array}$ \\
\hline
\end{tabular}


Table 2-2

(11 sheets)

\section{Hanford Site Radionuclide Air Emissions Data}

For Minor Point Sources.

(minor point sources have the potential of $<0.1 \mathrm{mrem} / \mathrm{y}$ EDE to nearest offsite resident)

\begin{tabular}{|c|c|c|c|c|c|c|}
\hline $\begin{array}{c}\text { Source } \mathrm{ID}^{\mathrm{a}} \\
\text { (facility/building) } \\
\text { [contractor] }\end{array}$ & $\begin{array}{c}\text { Discharge } \\
\text { Height } \\
\text { (m) }\end{array}$ & $\begin{array}{l}\text { Emission } \\
\text { Control }^{b}\end{array}$ & $\begin{array}{l}\text { Total } \\
\text { Flow } \\
\left(\mathbf{m}^{3}\right)\end{array}$ & $\begin{array}{c}\text { Annual } \\
\text { Average } \\
\text { Flowrate } \\
\left(\mathbf{m}^{3} / \mathbf{s}\right)\end{array}$ & Radionuclide & $\begin{array}{c}\text { Annual } \\
\text { Emissions } \\
(\mathrm{Ci})^{\mathbf{c}}\end{array}$ \\
\hline $\begin{array}{l}\text { 296-A-26 } \\
\text { (East Tank Farms) } \\
\text { [WHC] }\end{array}$ & 9.4 & HEPA & $2.9 \mathrm{E}+07$ & 0.9 & $\begin{array}{l}\text { total alpha } \\
\text { total beta }\end{array}$ & $\begin{array}{l}7.2 \mathrm{E}-09 \\
2.4 \mathrm{E}-08\end{array}$ \\
\hline $\begin{array}{l}296-\mathrm{A}-27 \\
\text { (East Tank Farms) } \\
\text { [WHC] }\end{array}$ & 3.7 & HEPA & $1.8 \mathrm{E}+07$ & 0.6 & $\begin{array}{l}{ }^{90} \mathrm{Sr} \\
{ }^{106} \mathrm{Ru} \\
{ }^{113} \mathrm{Sn} \\
{ }^{125} \mathrm{Sb} \\
{ }^{129} \mathrm{I} \\
{ }^{137} \mathrm{Cs} \\
{ }^{238} \mathrm{Pu} \\
{ }^{239 / 240} \mathrm{Pu} \\
{ }^{241} \mathrm{Am}\end{array}$ & $\begin{array}{c}\text { ND } \\
1.3 \mathrm{E}-06 \\
1.2 \mathrm{E}-07 \\
3.5 \mathrm{E}-07 \\
1.4 \mathrm{E}-05 \\
2.5 \mathrm{E}-09 \\
\mathrm{ND} \\
1.7 \mathrm{E}-10 \\
8.2 \mathrm{E}-10\end{array}$ \\
\hline $\begin{array}{l}296-A-28 \\
\text { (East Tank Farms) } \\
\text { [WHC] }\end{array}$ & 3.7 & $\overrightarrow{\text { HEPA }}$ & $9.3 \mathrm{E}+07$ & 2.9 & $\begin{array}{l}\text { total alpha } \\
\text { total beta }\end{array}$ & $\begin{array}{l}4.6 \mathrm{E}-08 \\
3.9 \mathrm{E}-07\end{array}$ \\
\hline $\begin{array}{l}296-\text { A-29 } \\
\text { (East Tank Farms) } \\
\text { [WHC] }\end{array}$ & 3.7 & HEPA & $1.4 \mathrm{E}+07$ & 0.4 & $\begin{array}{l}{ }^{90} \mathrm{Sr} \\
{ }^{137} \mathrm{Cs} \\
{ }^{154} \mathrm{Eu} \\
{ }^{238} \mathrm{Pu} \\
{ }^{239 / 240} \mathrm{Pu} \\
{ }^{24 !} \mathrm{Am}\end{array}$ & $\begin{array}{l}2.0 \mathrm{E}-08 \\
1.9 \mathrm{E}-10 \\
9.4 \mathrm{E}-10 \\
3.0 \mathrm{E}-11 \\
1.1 \mathrm{E}-10 \\
1.1 \mathrm{E}-09\end{array}$ \\
\hline $\begin{array}{l}296-A-30 \\
\text { (East Tank Farms) } \\
\text { [WHC] }\end{array}$ & 3.7 & $\overline{\mathrm{HEPA}}$ & $9.2 E+07$ & 2.9 & $\begin{array}{l}\text { total alpha } \\
\text { total beta }\end{array}$ & $\begin{array}{l}2.1 \mathrm{E}-08 \\
1.8 \mathrm{E}-07\end{array}$ \\
\hline $\begin{array}{l}296-A-41 \\
\text { (East Tank Farms) } \\
\text { [WHC] }\end{array}$ & 8.9 & HEPA & $1.5 \mathrm{E}+08$ & 4.8 & $\begin{array}{l}\text { total alpha } \\
\text { total beta }\end{array}$ & $\begin{array}{l}2.4 \mathrm{E}-08 \\
1.1 \mathrm{E}-07\end{array}$ \\
\hline $\begin{array}{l}296-P-17 \\
\text { (East Tank Farms) } \\
{[\text { WHC] }}\end{array}$ & 4.6 & HEPA & $0.0 \mathrm{E}+00$ & 0.0 & \multicolumn{2}{|c|}{ (did not operate) } \\
\hline $\begin{array}{l}\text { 296-P-31 } \\
\text { (East Tank Farms) } \\
\text { [WHC] }\end{array}$ & 10.0 & HEPA & $3.3 \mathrm{E}+07$ & 1.0 & $\begin{array}{l}\text { total alpha } \\
\text { total beta }\end{array}$ & $\begin{array}{l}6.4 \text { E-09 } \\
4.2 \text { E-08 }\end{array}$ \\
\hline
\end{tabular}


Table 2-2

(11 sheets)

\begin{tabular}{|c|c|c|c|c|c|c|}
\hline \multicolumn{7}{|c|}{$\begin{array}{l}1995 \text { Hanford Site Radionuclide Air Emissions Data } \\
\text { For Minor Point Sources. } \\
\text { (minor point sources have the potential of }<0.1 \text { mrem/y EDE to nearest offsite resident) }\end{array}$} \\
\hline $\begin{array}{l}\text { Source } \mathbf{I D}^{\mathrm{a}} \\
\text { (facility/building) } \\
\text { [contractor] }\end{array}$ & $\begin{array}{l}\text { Discharge } \\
\text { Height } \\
\text { (m) }\end{array}$ & $\begin{array}{l}\text { Emission } \\
\text { Control }^{\mathrm{b}}\end{array}$ & $\begin{array}{l}\text { Total } \\
\text { Flow } \\
\left(\mathrm{m}^{3}\right)\end{array}$ & $\begin{array}{c}\text { Annual } \\
\text { Average } \\
\text { Flowrate } \\
\left(\mathbf{m}^{3} / \mathbf{s}\right)\end{array}$ & Radionuclide & $\begin{array}{c}\text { Annual } \\
\text { Emissions } \\
\text { (Ci) }^{c}\end{array}$ \\
\hline $\begin{array}{l}\text { 296-A-21 } \\
\text { (242-A Evaporator) } \\
{[\text { WHC] }}\end{array}$ & 6.7 & HEPA & $3.4 \mathrm{E}+08$ & 10.7 & $\begin{array}{l}{ }^{90} \mathrm{Sr} \\
{ }^{106} \mathrm{Ru} \\
{ }^{125} \mathrm{Sb} \\
{ }^{137} \mathrm{Cs} \\
{ }^{152} \mathrm{Eu} \\
{ }^{154} \mathrm{Eu} \\
{ }^{155} \mathrm{Eu} \\
{ }^{238} \mathrm{Pu} \\
{ }^{239 / 240} \mathrm{Pu} \\
{ }^{241} \mathrm{Am}\end{array}$ & $\begin{array}{l}7.3 \mathrm{E}-07 \\
4.0 \mathrm{E}-08 \\
7.6 \mathrm{E}-09 \\
8.5 \mathrm{E}-09 \\
2.3 \mathrm{E}-09 \\
7.1 \mathrm{E}-08 \\
1.6 \mathrm{E}-09 \\
1.1 \mathrm{E}-09 \\
1.4 \mathrm{E}-08 \\
1.1 \mathrm{E}-08\end{array}$ \\
\hline $\begin{array}{l}296-E-1 \\
\text { (ETF) } \\
{[W H C]}\end{array}$ & 15.5 & HEPA & $1.2 \mathrm{E}+08$ & 3.7 & $\begin{array}{l}\text { total alpha } \\
\text { total beta }\end{array}$ & $\begin{array}{l}9.6 \mathrm{E}-08 \\
2.1 \mathrm{E}-07\end{array}$ \\
\hline $\begin{array}{l}\text { 296-G-1 } \\
\text { (Grout) } \\
\text { [WHC] }\end{array}$ & 7.6 & HEPA & $0.0 \mathrm{E}+00$ & 0.0 & (did not & erate) \\
\hline \multicolumn{7}{|c|}{200 West Area Point Sources } \\
\hline $\begin{array}{l}\text { 296-P-22 } \\
\text { (West Tank Farms) } \\
{[\text { WHC] }}\end{array}$ & 4.6 & HEPA & $1.8 \mathrm{E}+07$ & 0.6 & $\begin{array}{l}\text { total alpha } \\
\text { total beta }\end{array}$ & $\begin{array}{l}1.7 \mathrm{E}-09 \\
1.8 \mathrm{E}-08\end{array}$ \\
\hline $\begin{array}{l}296-P-23 \\
296-P-28 \text { (backup) } \\
\text { (West Tank Farms) } \\
\text { [WHC] }\end{array}$ & 4.6 & HEPA & $1.8 \mathrm{E}+07$ & 0.6 & $\begin{array}{l}{ }^{90} \mathrm{Sr} \\
{ }^{106} \mathrm{Ru} \\
{ }^{113} \mathrm{Sn} \\
{ }^{125} \mathrm{Sb} \\
{ }^{134} \mathrm{Cs} \\
{ }^{137} \mathrm{Cs} \\
{ }^{152} \mathrm{Eu} \\
{ }^{155} \mathrm{Eu} \\
{ }^{238} \mathrm{Pu} \\
{ }^{239} 440 \mathrm{Pu} \\
{ }^{241} \mathrm{Am}\end{array}$ & $\begin{array}{l}1.3 \mathrm{E}-07 \\
4.0 \mathrm{E}-09 \\
6.0 \mathrm{E}-10 \\
3.4 \mathrm{E}-09 \\
4.0 \mathrm{E}-10 \\
4.0 \mathrm{E}-06 \\
1.7 \mathrm{E}-09 \\
2.4 \mathrm{E}-09 \\
3.9 \mathrm{E}-10 \\
1.7 \mathrm{E}-09 \\
3.9 \mathrm{E}-09\end{array}$ \\
\hline $\begin{array}{l}296-S-18 \\
\text { (West Tank Farms) } \\
\text { [WHC] }\end{array}$ & 6.7 & HEPA & $2.2 \mathrm{E}+08$ & 7.1 & $\begin{array}{l}\text { total alpha } \\
\text { total beta }\end{array}$ & $\begin{array}{l}3.8 \mathrm{E}-08 \\
6.4 \mathrm{E}-07\end{array}$ \\
\hline $\begin{array}{l}296-\mathrm{T}-17 \\
\text { (West Tank Farms) } \\
\text { [WHC] }\end{array}$ & 10.1 & HEPA & $3.0 \mathrm{E}+07$ & 0.9 & $\begin{array}{l}\text { total alpha } \\
\text { total beta }\end{array}$ & $\begin{array}{l}2.2 \mathrm{E}-09 \\
2.8 \mathrm{E}-08\end{array}$ \\
\hline $\begin{array}{l}296-\text { W-3 } \\
\text { (West Tank Farms) } \\
\text { [WHC] }\end{array}$ & 7.6 & HEPA & $3.1 \mathrm{E}+07$ & 1.0 & $\begin{array}{l}\text { total alpha } \\
\text { total beta }\end{array}$ & $\begin{array}{l}1.4 \mathrm{E}-08 \\
5.9 \mathrm{E}-08\end{array}$ \\
\hline
\end{tabular}


Table 2-2

(11 sheets)

\begin{tabular}{|c|c|c|c|c|c|c|}
\hline \multicolumn{7}{|c|}{$\begin{array}{l}1995 \text { Hanford Site Radionuclide Air Emissions Data } \\
\text { For Minor Point Sources. } \\
\text { (minor point sources have the potential of }<0.1 \mathrm{mrem} / \mathrm{y} \text { EDE to nearest offsite resident) }\end{array}$} \\
\hline $\begin{array}{c}\text { Source } \mathrm{ID}^{\mathbf{a}} \\
\text { (facility/building) } \\
\text { [coatractor] }\end{array}$ & $\begin{array}{l}\text { Discharge } \\
\text { Height } \\
\text { (im) }\end{array}$ & $\begin{array}{l}\text { Emission } \\
\text { Control }^{\mathbf{b}}\end{array}$ & $\begin{array}{l}\text { Total } \\
\text { Flow } \\
\left(\mathrm{m}^{3}\right)\end{array}$ & $\begin{array}{c}\text { Annual } \\
\text { Average } \\
\text { Flowrate } \\
\left(\mathbf{m}^{3} / \mathbf{s}\right)\end{array}$ & Radionuclide & $\begin{array}{c}\text { Annual } \\
\text { Emissions } \\
\text { (Ci) }\end{array}$ \\
\hline $\begin{array}{l}\text { 291-S-1 } \\
\text { (S Plant/REDOX) } \\
\text { [BHI] }\end{array}$ & 61.0 & sand filter & $3.1 \mathrm{E}+08$ & 9.9 & $\begin{array}{l}\text { total alpha } \\
\text { total beta }\end{array}$ & $\begin{array}{l}2.1 \mathrm{E}-07 \\
6.7 \mathrm{E}-07\end{array}$ \\
\hline $\begin{array}{l}296-S-2 \\
\text { (S Plant/REDOX) } \\
{[\mathrm{BHI}]}\end{array}$ & 20.7 & HEPA & $1.9 \mathrm{E}+07$ & 0.6 & $\begin{array}{l}\text { total alpha } \\
\text { total beta }\end{array}$ & $\begin{array}{l}6.9 \mathrm{E}-09 \\
1.6 \mathrm{E}-08\end{array}$ \\
\hline $\begin{array}{l}296-S-4 \\
\text { (S Plant/REDOX) } \\
\text { [BHI] }\end{array}$ & 20.7 & HEPA & $4.4 \mathrm{E}+06$ & 0.1 & $\begin{array}{l}\text { total alpha } \\
\text { total beta }\end{array}$ & $\begin{array}{l}4.4 \mathrm{E}-09 \\
1.4 \mathrm{E}-08\end{array}$ \\
\hline $\begin{array}{l}\text { 296-S-6 } \\
\text { (S Plant/REDOX) } \\
\text { [BHI] }\end{array}$ & 39.4 & none & $1.2 \mathrm{E}+07$ & 0.4 & $\begin{array}{l}\text { total alpha } \\
\text { total beta }\end{array}$ & $\begin{array}{l}2.2 \mathrm{E}-08 \\
8.0 \mathrm{E}-08\end{array}$ \\
\hline $\begin{array}{l}296-S-7 W \\
296-S-7 E \text { (backup) } \\
\text { (S Plant/REDOX) } \\
\text { [BHI] }\end{array}$ & 7.6 & HEPA & $1.4 \mathrm{E}+08$ & 4.3 & $\begin{array}{l}\text { total alpha } \\
\text { total beta }\end{array}$ & $\begin{array}{l}5.6 \mathrm{E}-07 \\
2.9 \mathrm{E}-07\end{array}$ \\
\hline $\begin{array}{l}\text { 291-U-1 } \\
\text { (U Plant) } \\
\text { [BHI] }\end{array}$ & 61.0 & sand filter & $3.7 \mathrm{E}+08$ & 11.8 & $\begin{array}{l}\text { total alpha } \\
\text { total beta }\end{array}$ & $\begin{array}{l}2.5 \mathrm{E}-07 \\
5.4 \mathrm{E}-05\end{array}$ \\
\hline $\begin{array}{l}291-\mathrm{T}-1 \\
\text { (T Plant) } \\
\text { [WHC] }\end{array}$ & 61.0 & HEPA & $5.2 E+08$ & 16.6 & $\begin{array}{l}{ }^{90} \mathrm{Sr} \\
{ }^{125} \mathrm{Sb} \\
{ }^{134} \mathrm{Cs} \\
{ }^{137} \mathrm{Cs} \\
{ }^{152} \mathrm{Eu} \\
{ }^{154} \mathrm{Eu} \\
{ }^{155} \mathrm{Eu} \\
{ }^{238} \mathrm{Pu} \\
{ }^{239} / 240 \\
{ }^{241} \mathrm{Am}\end{array}$ & $\begin{array}{ll}2.7 & \mathrm{E}-05 \\
2.0 & \mathrm{E}-08 \\
1.7 & \mathrm{E}-08 \\
1.5 & \mathrm{E}-05 \\
5.2 & \mathrm{E}-08 \\
1.3 & \mathrm{E}-07 \\
1.1 & \mathrm{E}-07 \\
3.2 & \mathrm{E}-07 \\
2.4 & \mathrm{E}-05 \\
1.8 & \mathrm{E}-06\end{array}$ \\
\hline $\begin{array}{l}296-\mathrm{T}-7 \\
\text { (T Plant) } \\
\text { [WHC] }\end{array}$ & 8.5 & HEPA & $2.3 \mathrm{E}+07$ & 0.7 & $\begin{array}{l}\text { total alpha } \\
\text { total beta }\end{array}$ & $\begin{array}{l}5.0 \mathrm{E}-09 \\
4.6 \mathrm{E}-08\end{array}$ \\
\hline $\begin{array}{l}\text { 296-T-13 } \\
\text { (T Plant) } \\
\text { [WHC] }\end{array}$ & 20.7 & HEPA & $5.6 \mathrm{E}+08$ & 17.7 & $\begin{array}{l}\text { total alpha } \\
\text { total beta }\end{array}$ & $\begin{array}{l}1.1 \mathrm{E}-06 \\
8.8 \mathrm{E}-06\end{array}$ \\
\hline $\begin{array}{l}296-T-11 \\
\text { (TRUSAF) } \\
\text { [WHC] }\end{array}$ & 7.6 & HEPA & $5.8 \mathrm{E}+06$ & 0.2 & $\begin{array}{l}\text { total alpha } \\
\text { total beta }\end{array}$ & $\begin{array}{l}7.8 \mathrm{E}-10 \\
3.8 \mathrm{E}-09\end{array}$ \\
\hline
\end{tabular}


Table 2-2

(11 sheets)

\begin{tabular}{|c|c|c|c|c|c|c|}
\hline \multicolumn{7}{|c|}{$\begin{array}{l}1995 \text { Hanford Site Radionuclide Air Emissions Data } \\
\text { For Minor Point Sources. } \\
\text { (minor point sources have the potential of }<0.1 \text { mrem/y EDE to nearest offsite resident) }\end{array}$} \\
\hline $\begin{array}{c}\text { Source } \mathbf{W}^{\mathbf{2}} \\
\text { (facility/building) } \\
\text { [contractor] }\end{array}$ & $\begin{array}{l}\text { Discharge } \\
\text { Height } \\
\text { (m) }\end{array}$ & $\begin{array}{l}\text { Emission } \\
\text { Control }^{\mathbf{b}}\end{array}$ & $\begin{array}{l}\text { Total } \\
\text { Flow } \\
\left(\mathbf{m}^{3}\right)\end{array}$ & $\begin{array}{l}\text { Annual } \\
\text { Average } \\
\text { Flowrate } \\
\left(\mathrm{m}^{3} / \mathrm{s}\right)\end{array}$ & Radionuclide & $\begin{array}{c}\text { Annual } \\
\text { Emissions } \\
\text { (Ci) }^{c}\end{array}$ \\
\hline $\begin{array}{l}296-\mathrm{T}-12 \\
\text { (TRUSAF) } \\
\text { [WHC] }\end{array}$ & 7.6 & HEPA & $1.4 \mathrm{E}+08$ & 4.5 & $\begin{array}{l}\text { total alpha } \\
\text { total beta }\end{array}$ & $\begin{array}{l}1.2 \mathrm{E}-07 \\
7.9 \mathrm{E}-07\end{array}$ \\
\hline $\begin{array}{l}296-\mathrm{S}-16 \\
(222-\mathrm{S}) \\
{[\mathrm{WHC}]}\end{array}$ & 3.0 & HEPA & $1.6 \mathrm{E}+06$ & $<0.1$ & $\begin{array}{l}\text { total alpha } \\
\text { total beta }\end{array}$ & $\begin{array}{l}2.7 \mathrm{E}-08 \\
5.4 \mathrm{E}-07\end{array}$ \\
\hline $\begin{array}{l}296-S-21 \\
(222-S) \\
\text { [WHC] }\end{array}$ & 11.6 & $\overline{\text { HEPA }}$ & $1.1 E+09$ & 34.9 & $\begin{array}{l}{ }_{238 / 204}^{238} \mathrm{Pu} \\
{ }^{241} \mathrm{Am} \\
\text { total beta }\end{array}$ & $\begin{array}{l}7.1 \mathrm{E}-09 \\
8.0 \mathrm{E}-08 \\
4.5 \mathrm{E}-08 \\
5.8 \mathrm{E}-07\end{array}$ \\
\hline $\begin{array}{l}\overline{296-Z-3} \\
\text { (PFP) } \\
{[\text { WHC] }}\end{array}$ & 7.6 & HEPA & $1.4 \mathrm{E}+07$ & 0.4 & $\begin{array}{l}{ }^{238} \mathrm{Pu} \\
{ }^{239 / 240} \mathrm{Pu} \\
{ }^{241} \mathrm{Pu} \\
{ }^{241} \mathrm{Am} \\
\text { total beta }\end{array}$ & $\begin{array}{ll}3.5 & \mathrm{E}-08 \\
3.1 \mathrm{E}-08 \\
3.1 \mathrm{E}-06 \\
1.5 \mathrm{E}-08 \\
4.0 \mathrm{E}-08\end{array}$ \\
\hline $\begin{array}{l}\text { 296-Z-5 } \\
\text { (PFP) } \\
\text { [WHC] }\end{array}$ & $\overline{4.6}$ & HEPA & $1.4 \mathrm{E}+08$ & 4.4 & $\begin{array}{l}\text { total alpha } \\
\text { total beta }\end{array}$ & $\begin{array}{l}1.9 \mathrm{E}-08 \\
5.6 \mathrm{E}-08\end{array}$ \\
\hline $\begin{array}{l}296-Z-6 \\
\text { (PFP) } \\
{[W H C]}\end{array}$ & 0.9 & $\overline{\text { HEPA }}$ & $1.3 \mathrm{E}+08$ & 4.2 & $\begin{array}{l}\text { total alpha } \\
\text { total beta }\end{array}$ & $\begin{array}{l}4.1 \mathrm{E}-08 \\
1.6 \mathrm{E}-07\end{array}$ \\
\hline $\begin{array}{l}296-Z-14 \\
\text { (PFP) } \\
{[\mathrm{WHC}]}\end{array}$ & 6.1 & HEPA & $3.0 \mathrm{E}+07$ & 1.0 & $\begin{array}{l}\text { total alpha } \\
\text { total beta }\end{array}$ & $\begin{array}{l}8.6 \mathrm{E}-09 \\
4.9 \mathrm{E}-08\end{array}$ \\
\hline $\begin{array}{l}296-Z-15 \\
\text { (PFP) } \\
{[\mathrm{WHC}]}\end{array}$ & 3.6 & $\overline{\mathrm{HEPA}}$ & $2.4 \mathrm{E}+07$ & 0.8 & total alpha & $3.9 \mathrm{E}-09$ \\
\hline $\begin{array}{l}696-W-1 \\
\text { (WSCF) } \\
\text { [WHC] }\end{array}$ & 7.6 & HEPA & $7.4 E+08$ & 23.6 & $\begin{array}{l}\text { total alpha } \\
\text { total beta }\end{array}$ & $\begin{array}{l}9.4 \mathrm{E}-08 \\
5.0 \mathrm{E}-07\end{array}$ \\
\hline $\begin{array}{l}\text { 696-W-2 } \\
\text { (WSCF) } \\
\text { [WHC] }\end{array}$ & 9.8 & HEPA & $2.2 \mathrm{E}+07$ & 0.7 & $\begin{array}{l}\text { total alpha } \\
\text { total beta }\end{array}$ & $\begin{array}{l}6.1 \mathrm{E}-09 \\
3.6 \mathrm{E}-08\end{array}$ \\
\hline \multicolumn{7}{|c|}{300 Area Point Sources } \\
\hline $\begin{array}{l}\text { 306-E-ULAB } \\
\text { (306-E Bldg.) } \\
\text { [WHC] }\end{array}$ & 20.1 & $\overline{\text { HEPA }}$ & $4.4 \mathrm{E}+07$ & 1.4 & $\begin{array}{l}\text { total alpha } \\
\text { total beta }\end{array}$ & $\begin{array}{l}2.2 \mathrm{E}-08 \\
5.0 \mathrm{E}-08\end{array}$ \\
\hline
\end{tabular}


Table 2-2

(11 sheets)

\begin{tabular}{|c|c|c|c|c|c|c|}
\hline \multicolumn{7}{|c|}{$\begin{array}{l}1995 \text { Hanford Site Radionuclide Air Emissions Data } \\
\text { For Minor Point Sources. } \\
\text { (minor point sources have the potential of }<0.1 \mathrm{mrem} / \mathrm{y} \text { EDE to nearest offsite resident) }\end{array}$} \\
\hline $\begin{array}{l}\text { Source ID } \\
\text { (facility/building) } \\
\text { [contractor] }\end{array}$ & $\begin{array}{c}\text { Discharge } \\
\text { Height } \\
\text { (m) }\end{array}$ & $\begin{array}{l}\text { Emission } \\
\text { Centrol }^{\mathrm{b}}\end{array}$ & $\begin{array}{l}\text { Total } \\
\text { Flow } \\
\left(\mathbf{m}^{3}\right)\end{array}$ & $\begin{array}{c}\text { Arnuual } \\
\text { Average } \\
\text { Flowrate } \\
\left(\mathbf{m}^{3} / \mathrm{s}\right)\end{array}$ & Radionuclide & $\begin{array}{c}\text { Annual } \\
\text { Emissions } \\
\text { (Ci) }^{\mathbf{c}}\end{array}$ \\
\hline $\begin{array}{l}\text { 308-TRIGA } \\
\text { (308 Bldg.) } \\
\text { [WHC] }\end{array}$ & 11.3 & $\begin{array}{l}\text { HEPA, } \\
\text { charcoal }\end{array}$ & $2.7 \mathrm{E}+07$ & 0.9 & $\begin{array}{l}{ }^{131} \text { I } \\
\text { total alpha } \\
\text { total beta }\end{array}$ & $\begin{array}{l}\mathrm{ND} \\
5.3 \mathrm{E}-08 \\
1.2 \mathrm{E}-07\end{array}$ \\
\hline $\begin{array}{l}\text { 309-PRTR } \\
\text { (309 Bldg.) } \\
\text { [WHC] }\end{array}$ & 30.5 & HEPA & $3.5 \mathrm{E}+07$ & 1.1 & $\begin{array}{l}{ }^{60} \mathrm{Co} \\
{ }^{90} \mathrm{Sr} \\
{ }^{137} \mathrm{Cs} \\
{ }^{239} \mathrm{Pu}\end{array}$ & $\begin{array}{l}<3.7 \mathrm{E}-10 \\
<1.4 \mathrm{E}-10 \\
<3.3 \mathrm{E}-10 \\
<1.2 \mathrm{E}-10\end{array}$ \\
\hline $\begin{array}{l}\text { 309-RTF-EX } \\
\text { (309 Bldg.) } \\
\text { [WHC] }\end{array}$ & 4.0 & HEPA & $4.8 \mathrm{E}+06$ & 0.2 & ${ }^{137} \mathrm{Cs}$ & $<6.3 \mathrm{E}-12$ \\
\hline $\begin{array}{l}\text { EP-303C-01-S } \\
\text { (303-C Bldg.) } \\
\text { [PNNL] }\end{array}$ & 4.0 & HEPA & $1.0 \mathrm{E}+07$ & 0.3 & $\begin{array}{l}\text { total alpha } \\
\text { total beta }\end{array}$ & $\begin{array}{ll}6.1 & \mathrm{E}-09 \\
7.3 \mathrm{E}-08\end{array}$ \\
\hline $\begin{array}{l}\text { EP-306W-01-V } \\
\text { (306-W Bldg.) } \\
\text { [PNNL] }\end{array}$ & 8.0 & HEPA & $2.4 \mathrm{E}+08$ & 7.6 & $\begin{array}{l}\text { total alpha } \\
\text { total beta }\end{array}$ & $\begin{array}{l}6.2 \mathrm{E}-08 \\
7.5 \mathrm{E}-07\end{array}$ \\
\hline $\begin{array}{l}\text { EP-306W-02-V } \\
\text { (306-W Bldg.) } \\
\text { [PNNL] }\end{array}$ & 8.0 & HEPA & $4.1 \mathrm{E}+08$ & 13.0 & $\begin{array}{l}\text { total alpha } \\
\text { total beta }\end{array}$ & $\begin{array}{l}1.0 \mathrm{E}-07 \\
1.3 \mathrm{E}-06\end{array}$ \\
\hline $\begin{array}{l}\text { EP-306W-03-V } \\
\text { (306-W Bldg.) } \\
\text { [PNNL] }\end{array}$ & 8.0 & HEPA & $4.1 \mathrm{E}+08$ & 13.0 & $\begin{array}{l}\text { total alpha } \\
\text { total beta }\end{array}$ & $\begin{array}{l}5.6 \mathrm{E}-08 \\
5.9 \mathrm{E}-07\end{array}$ \\
\hline $\begin{array}{l}\text { EP-306W-04-S } \\
\text { (306-W BIdg.) } \\
\text { [PNNL] }\end{array}$ & 10.0 & HEPA. & $2.2 \mathrm{E}+08$ & 6.7 & $\begin{array}{l}\text { total alpha } \\
\text { total beta }\end{array}$ & $\begin{array}{l}4.7 \mathrm{E}-09 \\
8.2 \mathrm{E}-08\end{array}$ \\
\hline $\begin{array}{l}\text { 340-B-BLDG } \\
\text { (340 Bldg.) } \\
\text { [WHC] }\end{array}$ & 11.6 & HEPA & $5.0 \mathrm{E}+06$ & 0.2 & $\begin{array}{l}\text { total alpha } \\
\text { total beta }\end{array}$ & $\begin{array}{l}\text { ND } \\
\text { ND }\end{array}$ \\
\hline $\begin{array}{l}\text { 340-DECON } \\
\text { (340 Bldg.) } \\
\text { [WHC] }\end{array}$ & 3.0 & HEPA & $1.1 \mathrm{E}+08$ & 3.4 & $\begin{array}{l}\text { total alpha } \\
\text { total beta }\end{array}$ & $\begin{array}{ll}2.7 & \mathrm{E}-07 \\
8.5 & \mathrm{E}-07\end{array}$ \\
\hline $\begin{array}{l}377-\text { GEL } \\
\text { (377 Bldg.) } \\
{[\text { WHC] }}\end{array}$ & 10.0 & HEPA & $1.2 \mathrm{E}+08$ & 3.9 & $\begin{array}{l}\text { total alpha } \\
\text { total beta }\end{array}$ & $\begin{array}{l}\text { ND } \\
\text { ND }\end{array}$ \\
\hline $\begin{array}{l}\text { EP-314-01-S } \\
\text { (314 Bldg.) } \\
\text { [PNNL] }\end{array}$ & 6.0 & HEPA & $4.9 \mathrm{E}+07$ & 1.6 & $\begin{array}{l}\text { total alpha } \\
\text { total beta }\end{array}$ & $\begin{array}{l}1.2 \mathrm{E}-08 \\
1.2 \mathrm{E}-07\end{array}$ \\
\hline
\end{tabular}


Table 2-2

(11 sheets)

\section{Hanford Site Radionuclide Air Emissions Data}

For Minor Point Sources.

(minor point sources have the potential of $<0.1 \mathrm{mrem} / \mathrm{y}$ EDE to nearest offsite resident)

\begin{tabular}{|c|c|c|c|c|c|c|}
\hline $\begin{array}{l}\text { Source ID" } \\
\text { (facility/building) } \\
\text { [contractor] }\end{array}$ & $\begin{array}{l}\text { Discharge } \\
\text { Height } \\
\text { (m) }\end{array}$ & $\begin{array}{l}\text { Emission } \\
\text { Control }^{b}\end{array}$ & $\begin{array}{l}\text { Total } \\
\text { Flow } \\
\left(\mathbf{m}^{3}\right)\end{array}$ & $\begin{array}{c}\text { Annual } \\
\text { Average } \\
\text { Flowrate } \\
\left(\mathbf{m}^{3} / \mathbf{s}\right)\end{array}$ & Radionuclide & $\begin{array}{c}\text { Annual } \\
\text { Emissions } \\
\text { (Ci) }^{c}\end{array}$ \\
\hline $\begin{array}{l}\text { EP-318-01-S } \\
\text { (318 Bldg.) } \\
\text { [PNNL] }\end{array}$ & 9.0 & None & $9.6 \mathrm{E}+07$ & 3.0 & $\begin{array}{l}\text { total alpha } \\
\text { total beta }\end{array}$ & $\begin{array}{l}3.5 \mathrm{E}-08 \\
4.0 \mathrm{E}-07\end{array}$ \\
\hline $\begin{array}{l}\text { EP-320-01-S } \\
\text { (320 BIdg.) } \\
\text { [PNNL] }\end{array}$ & 13.0 & HEPA & $6.6 \mathrm{E}+08$ & 20.9 & $\begin{array}{l}\text { total alpha } \\
\text { total beta }\end{array}$ & $\begin{array}{l}1.9 \mathrm{E}-07 \\
2.3 \mathrm{E}-06\end{array}$ \\
\hline $\begin{array}{l}\text { EP-320-02-S } \\
\text { (320 Bldg.) } \\
\text { [PNNL] }\end{array}$ & 8.0 & HEPA & $7.9 \mathrm{E}+06$ & 0.3 & $\begin{array}{l}\text { total alpha } \\
\text { total beta }\end{array}$ & $\begin{array}{l}1.8 \mathrm{E}-09 \\
1.8 \mathrm{E}-08\end{array}$ \\
\hline $\begin{array}{l}\text { EP-320-03-S } \\
\text { (320 Bldg.) } \\
\text { [PNNL] }\end{array}$ & 6.0 & HEPA & $8.4 \mathrm{E}+06$ & 0.3 & $\begin{array}{l}\text { total alpha } \\
\text { total beta }\end{array}$ & $\begin{array}{l}9.8 \mathrm{E}-10 \\
1.3 \mathrm{E}-08\end{array}$ \\
\hline $\begin{array}{l}\text { EP-320-04-S } \\
\text { (320 Bldg.) } \\
\text { (PNNL) }\end{array}$ & 6.0 & $\overline{\text { HEPA }}$ & $8.0 \mathrm{E}+06$ & 0.3 & $\begin{array}{l}\text { total alpha } \\
\text { total beta }\end{array}$ & $\begin{array}{l}3.4 \mathrm{E}-10 \\
9.0 \mathrm{E}-09\end{array}$ \\
\hline $\begin{array}{l}\text { EP-323-01-S } \\
\text { (323 Bldg.) } \\
\text { [PNNL] }\end{array}$ & 5.0 & $\overline{\text { HEPA }}$ & $7.3 \mathrm{E}+07$ & 2.3 & $\begin{array}{l}\text { total aipha } \\
\text { total beta }\end{array}$ & $\begin{array}{l}2.0 \mathrm{E}-08 \\
1.1 \mathrm{E}-07\end{array}$ \\
\hline $\begin{array}{l}\text { EP-326-01-S } \\
\text { (326 Bidg.) } \\
\text { [PNNL] }\end{array}$ & 14.0 & HEPA & $7.8 \mathrm{E}+08$ & 24.7 & $\begin{array}{l}\text { total alpha } \\
\text { total beta }\end{array}$ & $\begin{array}{l}5.9 \mathrm{E}-07 \\
6.7 \mathrm{E}-06\end{array}$ \\
\hline $\begin{array}{l}\text { EP-329-01-S } \\
\text { (329 Bldg.) } \\
\text { [PNNL] }\end{array}$ & 11.0 & HEPA & $6.2 \mathrm{E}+08$ & 19.7 & $\begin{array}{l}\text { total alpha } \\
\text { total beta }\end{array}$ & $\begin{array}{l}2.5 \mathrm{E}-08 \\
4.1 \mathrm{E}-08\end{array}$ \\
\hline $\begin{array}{l}\text { EP-329-02-V } \\
\text { (329 Bldg.) } \\
\text { [PNNL] }\end{array}$ & 10.0 & HEPA & $6.7 \mathrm{E}+07$ & 2.1 & $\begin{array}{l}\text { total alpha } \\
\text { total beta }\end{array}$ & $\begin{array}{l}2.1 \mathrm{E}-08 \\
4.3 \mathrm{E}-07\end{array}$ \\
\hline $\begin{array}{l}\text { EP-331A-01-S } \\
\text { (331 Bldg.) } \\
\text { [PNNL] }\end{array}$ & 5.0 & HEPA & $9.2 \mathrm{E}+07$ & 2.9 & $\begin{array}{l}\text { cotal alpha } \\
\text { total beta }\end{array}$ & $\begin{array}{c}\mathrm{ND} \\
1.6 \mathrm{E}-08\end{array}$ \\
\hline $\begin{array}{l}\text { EP-331-01-V } \\
\text { (331 Bldg.) } \\
\text { [PNNL] }\end{array}$ & 15.0 & HEPA & $1.1 \mathrm{E}+09$ & 34.9 & $\begin{array}{l}\text { rotal alpha } \\
\text { total beta }\end{array}$ & $\begin{array}{l}4.1 \mathrm{E}-08 \\
3.5 \mathrm{E}-07\end{array}$ \\
\hline $\begin{array}{l}\text { EP-331A-02-V } \\
\text { (331 Bldg.) } \\
\text { [PNNL] }\end{array}$ & 6.0 & HEPA & $1.0 \mathrm{E}+07$ & 0.3 & $\begin{array}{l}\text { total alpha } \\
\text { total beta }\end{array}$ & $\begin{array}{c}5.1 \mathrm{E}-10 \\
\mathrm{ND}\end{array}$ \\
\hline $\begin{array}{l}\text { EP-331H-01-S } \\
\text { (331 Bldg.) } \\
\text { [PNNL] }\end{array}$ & 6.0 & HEPA & $1.1 \mathrm{E}+08$ & 3.5 & $\begin{array}{l}\text { total alpha } \\
\text { total beta }\end{array}$ & $\begin{array}{l}9.3 \mathrm{E}-09 \\
3.6 \mathrm{E}-08\end{array}$ \\
\hline
\end{tabular}


Table 2-2

(11 sheets)

\begin{tabular}{|c|c|c|c|c|c|c|}
\hline \multicolumn{7}{|c|}{$\begin{array}{l}1995 \text { Hanford Site Radionuclide Air Emissions Data } \\
\text { For Minor Point Sources. } \\
\text { (minor point sources have the potential of }<0.1 \text { mrem/y EDE to nearest offsite resident) }\end{array}$} \\
\hline $\begin{array}{c}\text { Source ID' } \\
\text { (facility/building) } \\
\text { [contractor] }\end{array}$ & $\begin{array}{l}\text { Discharge } \\
\text { Height } \\
\text { (m) }\end{array}$ & $\begin{array}{l}\text { Emission } \\
\text { Control }^{b}\end{array}$ & $\begin{array}{l}\text { Total } \\
\text { Flow } \\
\left(\mathbf{m}^{3}\right)\end{array}$ & $\begin{array}{c}\text { Annual } \\
\text { Average } \\
\text { Flowrate } \\
\left(\mathbf{m}^{3} / \mathbf{s}\right)\end{array}$ & Radionuclide & $\begin{array}{c}\text { Annual } \\
\text { Emissions } \\
\text { (Ci) }^{\mathfrak{c}}\end{array}$ \\
\hline $\begin{array}{l}\text { EP-3708-01-S } \\
\text { (3708 Bldg.) } \\
\text { [PNNL] }\end{array}$ & 7.0 & HEPA & $9.3 \mathrm{E}+07$ & 2.9 & $\begin{array}{l}\text { total alpha } \\
\text { total beta }\end{array}$ & $\begin{array}{l}1.6 \mathrm{E}-08 \\
1.1 \mathrm{E}-07\end{array}$ \\
\hline $\begin{array}{l}\text { EP-3720-02-S } \\
(3720 \text { Bldg. } \\
\text { [PNNL] }\end{array}$ & 5.0 & HEPA & $5.5 \mathrm{E}+07$ & 1.7 & $\begin{array}{l}\text { total alpha } \\
\text { total beta }\end{array}$ & $\begin{array}{l}3.6 \overline{\mathrm{E}-09} \\
7.3 \mathrm{E}-08\end{array}$ \\
\hline $\begin{array}{l}\text { EP-3720-03-S } \\
(3720 \text { Bldg.) } \\
{[\text { PNNL] }}\end{array}$ & 9.0 & HEPA & $9.2 \mathrm{E}+07$ & 2.9 & $\begin{array}{l}\text { total alpha } \\
\text { total beta }\end{array}$ & $\begin{array}{c}\mathrm{ND} \\
2.6 \mathrm{E}-08\end{array}$ \\
\hline $\begin{array}{l}\text { EP-3730-01-S } \\
(3730 \text { Bldg.) } \\
\text { [PNNL] }\end{array}$ & 5.0 & HEPA & $5.0 \mathrm{E}+06$ & 0.2 & $\begin{array}{l}\text { total alpha } \\
\text { total beta }\end{array}$ & $\begin{array}{l}3.0 \mathrm{E}-10 \\
7.7 \mathrm{E}-09\end{array}$ \\
\hline $\begin{array}{l}\text { EP-3745-01-S } \\
\text { (3745 Bldg.) } \\
\text { [PNNL] }\end{array}$ & 2.0 & HEPA & $6.6 E+06$ & 0.2 & $\begin{array}{l}\text { total alpha } \\
\text { total beta }\end{array}$ & $\begin{array}{l}2.5 \mathrm{E}-09 \\
2.3 \mathrm{E}-08\end{array}$ \\
\hline $\begin{array}{l}\text { EP-3746A-01-V } \\
\text { (3746 Bldg.) } \\
{[\text { [PNNL] }}\end{array}$ & 4.0 & HEPA & $5.5 \mathrm{E}+07$ & 1.7 & $\begin{array}{l}\text { total alpha } \\
\text { total beta }\end{array}$ & $\begin{array}{c}\text { ND } \\
3.5 \mathrm{E}-08\end{array}$ \\
\hline \multicolumn{7}{|c|}{400 Area Point Sources } \\
\hline $\begin{array}{l}\text { FFTF-CB-EX } \\
\text { (FFTF) } \\
\text { [WHC] }\end{array}$ & 14.3 & none & $3.2 \mathrm{E}+08$ & 10.2 & $\begin{array}{l}{ }^{3} \mathrm{H} \text { (as HTO) } \\
{ }^{331} \mathrm{I} \\
\text { total alpha } \\
\text { total beta }\end{array}$ & $\begin{array}{c}2.5 \mathrm{E}-02 \\
\mathrm{ND} \\
4.9 \mathrm{E}-07 \\
1.4 \mathrm{E}-06\end{array}$ \\
\hline $\begin{array}{l}\text { FFTF-RE-SB } \\
\text { (FFTF) } \\
\text { [WHC] }\end{array}$ & 6.1 & none & $2.7 \mathrm{E}+08$ & 8.7 & $\begin{array}{l}\text { total alpha } \\
\text { total beta }\end{array}$ & $\begin{array}{c}\mathrm{ND} \\
8.1 \mathrm{E}-07 \\
3.2 \mathrm{E}-06 \\
\end{array}$ \\
\hline $\begin{array}{l}\text { FFTF-HT-TR } \\
\text { (FFTF) } \\
\text { [WHC] }\end{array}$ & $\overline{8.8}$ & none & $6.7 \mathrm{E}+07$ & 2.1 & $\begin{array}{l}\text { total alpha } \\
\text { total beta }\end{array}$ & $\begin{array}{ll}1.1 & \mathrm{E}-07 \\
3.5 & \mathrm{E}-07\end{array}$ \\
\hline $\begin{array}{l}\text { 437-MN\&ST } \\
\text { (MASF) } \\
\text { [WHC] }\end{array}$ & 9.1 & none & $2.1 \mathrm{E}+08$ & 6.6 & $\begin{array}{l}\text { total alpha } \\
\text { total beta }\end{array}$ & $\begin{array}{l}3.2 \mathrm{E}-07 \\
9.5 \mathrm{E}-07\end{array}$ \\
\hline $\begin{array}{l}437-1-61 \\
\text { (MASF) } \\
{[\text { WHC] }}\end{array}$ & 11.7 & HEPA & $2.4 \mathrm{E}+08$ & 7.6 & $\begin{array}{l}\text { total alpha } \\
\text { total beta }\end{array}$ & $\begin{array}{l}\mathrm{ND} \\
\mathrm{ND}\end{array}$ \\
\hline
\end{tabular}


Table 2-2

(11 sheets)

\begin{tabular}{|c|c|c|c|c|c|c|}
\hline \multicolumn{7}{|c|}{$\begin{array}{l}1995 \text { Hanford Site Radionuclide Air Emissions Data } \\
\text { For Minor Point Sources. } \\
\text { (minor point sources have the potential of }<0.1 \mathrm{mrem} / \mathrm{y} \text { EDE to nearest offsite resident) }\end{array}$} \\
\hline $\begin{array}{l}\text { Source } \text { ID }^{\prime \prime} \\
\text { (facility/building) } \\
\text { [contractor] }\end{array}$ & $\begin{array}{l}\text { Discharge } \\
\text { Height } \\
\text { (m) }\end{array}$ & $\begin{array}{l}\text { Emission } \\
\text { Control }^{b}\end{array}$ & $\begin{array}{l}\text { Total } \\
\text { Flow } \\
\left(\mathrm{m}^{3}\right)\end{array}$ & $\begin{array}{l}\text { Annual } \\
\text { Average } \\
\text { Flowrate }^{\mathrm{e}} \\
\left(\mathrm{m}^{3} / \mathrm{s}\right)\end{array}$ & Radionuclide & $\begin{array}{c}\text { Annual } \\
\text { Emissions } \\
\text { (Ci) }^{c}\end{array}$ \\
\hline \multicolumn{7}{|c|}{600 Area Point Sources } \\
\hline $\begin{array}{l}\text { EP-6652H-01-V } \\
(6652-\mathrm{H} \text { Bldg.) } \\
\text { [PNNL] }\end{array}$ & 3.6 & HEPA & $5.0 \mathrm{E}+07$ & $1 . \overline{6}$ & $\begin{array}{l}\text { total alpha } \\
\text { total beta }\end{array}$ & $\begin{array}{l}2.8 \mathrm{E}-09 \\
6.1 \mathrm{E}-08\end{array}$ \\
\hline
\end{tabular}

Notes:

a $\mathrm{ID}=$ Identification, i.e., the alpha-mmeric designator for the respective point source; WHC = Westinghouse Hanford Company; PNNL = Pacific Northwest National Laboratory (Battelle); BHI = Bechtel Hanford, Inc.

b Efficiencies are: $\geq 99.95 \%$ for HEPA; $\geq 95 \%$ for charcoal; $\geq 99.8 \%$ for sand filter; $0 \%$ for no emission control.

c 1 Curie $=3.7 \mathrm{E}+10$ becquerel; $\mathrm{ND}=$ none detected (i.e. either the radionuclide was not detected in any sample during the year, or the average of all the measurements for that given radionuclide or rype of radioactivity made during the year was below background levels).

d Point Source emissions estimated from assessment data and confirmed with periodic confirmatory measurements. Confirmatory measurement data is provided in Section 5.0

e The annual average flowrate is calculated by dividing the total flow (discharge volume) by the number of seconds in 365 days. 
DOE/RL-96-37

This page intentionally left blank. 
Table 2-3

\begin{tabular}{|c|c|c|c|c|c|c|}
\hline \multicolumn{7}{|c|}{$\begin{array}{l}\text { Distances and Directions from Area Release Locations } \\
\text { to Nearest Receptors. }\end{array}$} \\
\hline \multirow{2}{*}{\multicolumn{2}{|c|}{ Category }} & \multicolumn{5}{|c|}{$\begin{array}{l}\text { Distances and Directions from Operating Areas } \\
\text { (mi) }\end{array}$} \\
\hline & & 100 Area & 200 East & 200 West & 300 Area & 400 Area \\
\hline \multirow{2}{*}{$\begin{array}{l}\text { Nearest: } \\
\text { residence, } \\
\text { school, business, } \\
\text { or office }\end{array}$} & $\begin{array}{l}\text { Nearest } \\
\text { Location }\end{array}$ & $7.0 \mathrm{NNW}$ & $13.2 \mathrm{E}$ & $8.5 \mathrm{~W}$ & $0.9 \mathrm{NE}$ & $5.5 \mathrm{E}$ \\
\hline & \begin{tabular}{|l|} 
Nearest in a \\
Prevailing Wind \\
Direction
\end{tabular} & $15.1 \mathrm{E}$ & $13.2 \mathrm{E}$ & $16.9 \mathrm{SE}$ & $2.1 \mathrm{~S}$ & $6.7 \mathrm{SE}$ \\
\hline \multirow{2}{*}{$\begin{array}{l}\text { Nearest: farm } \\
\text { producing } \\
\text { vegetables }\end{array}$} & $\begin{array}{l}\text { Nearest } \\
\text { Location }\end{array}$ & $6.1 \mathrm{NW}$ & $13.1 \mathrm{E}$ & $11.0 \mathrm{NW}$ & $2.0 \mathrm{E}$ & $6.5 \mathrm{ESE}$ \\
\hline & \begin{tabular}{|l|} 
Nearest in a \\
Prevailing Wind \\
Direction
\end{tabular} & $15.5 \mathrm{E}$ & $13.1 \mathrm{E}$ & $\begin{array}{c}18.6 \mathrm{E} \\
\& \\
18.6 \mathrm{SE}\end{array}$ & $2.5 \mathrm{NE}$ & $7.8 \mathrm{SE}$ \\
\hline \multirow[t]{2}{*}{$\begin{array}{l}\text { Nearest: farm } \\
\text { producing milk }\end{array}$} & $\begin{array}{l}\text { Nearest } \\
\text { Location }\end{array}$ & $21.7 \mathrm{E}$ & $18.1 \mathrm{ENE}$ & $24.2 \mathrm{ESE}$ & $3.6 \mathrm{SES}$ & $8.3 \mathrm{E}$ \\
\hline & \begin{tabular}{|l|} 
Nearest in a \\
Prevailing Wind \\
Direction
\end{tabular} & $21.7 \mathrm{E}$ & $19.0 \mathrm{ESE}$ & $21.5 \mathrm{~S}$ & $5.7 \mathrm{NE}$ & $9.5 \overline{\mathrm{SE}}$ \\
\hline \multirow[t]{2}{*}{$\begin{array}{l}\text { Nearest: farm } \\
\text { producing meat }\end{array}$} & $\begin{array}{l}\text { Nearest } \\
\text { Location }\end{array}$ & $7.0 \mathrm{NNW}$ & $13.0 \mathrm{WNW}$ & $11.0 \mathrm{WSW}$ & $1.7 \mathrm{ESE}$ & $7.6 \mathrm{SE}$ \\
\hline & \begin{tabular}{|l||} 
Nearest in a \\
Prevailing Wind \\
Direction
\end{tabular} & $19.5 \mathrm{ESE}$ & $14.9 \mathrm{E}$ & $16.8 \mathrm{SE}$ & $5.0 \mathrm{NE}$ & $7.6 \mathrm{SE}$ \\
\hline
\end{tabular}


DOE/RL-96-37

This page intentionally left blank. 


\subsection{POINT SOURCE EMISSION DOSE ASSESSMENTS}

\subsection{DESCRIPTION OF POINT SOURCE EMISSIONS DOSE MODEL}

The CAP88-PC computer code (Parks 1992) was used to demonstrate compliance with the $10 \mathrm{mrem} / \mathrm{y}$ EDE standard contained in 40 CFR Part 61.92. Because the Hanford site has numerous release points that are widely separated from each other, it was necessary to determine the point at which the maximum dose would be received from combined air emissions at all locations. Emission points used for modeling purposes were centered on each of the major Hanford Site operating areas that released radionuclides to the atmosphere during 1995. The reference point for the 100 Areas was the spent fuel storage basin at the 105-KE building. In the case of the 200 Areas, the reference points were the major sources in the 200 East and 200 West areas, the PUREX facility and PFP, respectively. Reference locations for the 300 Area were the 324 building, for non-radon emissions, and the 327 building, for ${ }^{220} \mathrm{Rn}$ and ${ }^{222} \mathrm{Rn}$ emissions. The Fast Flux Test Facility (FFTF) was used as the 400 Area reference location.

Point source emissions from the major operating areas are listed in Table 3-1. The location of the maximally exposed individual (MEI) selected for the compliance analysis was a farm at Sagemore Road, which is directly across the Columbia River from the site boundary to the east of the 300 Area. Table 3-2 lists the effective dose equivalent to this receptor from 1995 releases of individual radionuclides at each location, and the location of the emission sources relative to the Sagemore Road receptor.

\subsection{SUMMARY OF INPUT PARAMETERS}

Dose calculations were performed using established standard parameters for the Hanford site and its environment (PNL 1993). Release estimates, grouped by major operating area, were used to perform dose calculations, Table 3-1. Releases from point sources were modeled using an effective discharge height of $10 \mathrm{~m}$ for all release locations other than the 200 Area facilities, which were assumed to have an effective release height of $89 \mathrm{~m}$. Releases reported as total alpha or total beta were generally evaluated as ${ }^{239} \mathrm{Pu}$ or ${ }^{90} \mathrm{Sr}$, respectively, to provide a conservative estimate of the expected impact. In the case of 300 Area, some releases reported as total alpha measurements were modeled as depleted uranium, using facility radionuclide inventories as a basis. In the case of 400 Area, some releases reported as total beta measurements were modeled as ${ }^{137} \mathrm{Cs}$, based on facility specific information. 
Radionuclide data used for the dose calculations are listed in Appendix A; all other radionuclide-specific parameters were the default values provided by CAP88-PC data libraries. The maximum individual exposure and consumption parameters were those determined previously for the Hanford Site, and are also included in Appendix A.

Parameters used for the ingestion pathway assumed that the receptor's entire diet was produced at the residence location, the "local" food production option in CAP88-PC.

Radionuclide air concentrations at the receptor location were determined using site-specific meteorological data for each release point. Joint frequency distributions and CAP88-PC wind files were prepared from data collected at weather stations in each of the Hanford operating areas, and these represent the average of hourly data taken during 1995. This information was used to determine 1995 annual average dispersion coefficients for each of the major release points, see Appendix A.

\subsection{COMPLIANCE ASSESSMENT}

The combined dose to a receptor at the Sagemore farm from air emissions at all Hanford operating areas during 1995 was 0.0029 mrem EDE, which is less than $0.03 \%$ of the $10 \mathrm{mrem} / \mathrm{y}$ standard. As shown in Table 3-2, this dose resulted mainly from emissions related to activities at facilities in the 200 East Area. Operations at the 200 East area accounted for approximately $72 \%$ of the offsite dose, and the contributions from 200 West and 300 Area facilities made up most of the remainder. The 100 and 400 Area emissions contributed less than $2 \%$ of the total. Radionuclides, other than radon and thoron, contributing more than $10 \%$ of the total dose included ${ }^{3} \mathrm{H}(19.47 \%),{ }^{129} \mathrm{I}(61.51 \%)$, and ${ }^{239 / 240} \mathrm{Pu}(12.15 \%)$.

The MEI for the Hanford Site compliance calculations is located approximately 1.5 $\mathrm{km}$ east of the 300 Area facilities, based on a hypothetical release point at the 324 Building. The 324 Building was chosen to represent the source of all 300 Area non-radon emissions because it is the closest major emissions source in the 300 Area. The Sagemore Road location was selected as the MEI for the Site, because an individual at that location would also receive the maximum dose from radon and thoron emissions as well as from liquid effluents released to the Columbia River.

Again for emphasis:

HANFORD SITE EDE FOR 1995: 0.0029 mrem 
This dose is slightly lower than the annual dose for the previous years. The 1991 and 1992 doses were modeled using the mainframe version of CAP88 and an MEI location at Ringold. The 1993 through 1995 doses were modeled using CAP88-PC, which relies on default parameters more than the mainframe version. Figure 3-1 displays the offsite MEI doses attributable to Hanford Site emissions since 1990, the first year this report was issued.

Figure 3-1. Historical Effective Dose Equivalents to the MEI.

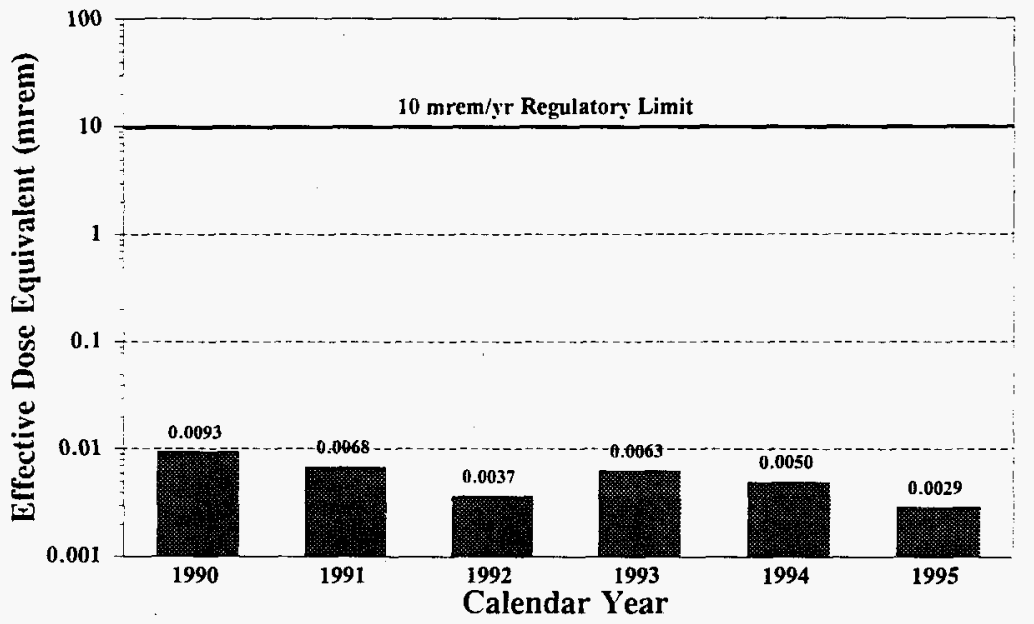


Table 3-1

\begin{tabular}{|c|c|c|c|c|c|c|}
\hline \multicolumn{7}{|c|}{$\begin{array}{l}\text { Release Estimates of Hanford Site } \\
\text { Radionuclide Air Emissions in } 1995 \text {. }\end{array}$} \\
\hline \multirow[b]{2}{*}{ Radionuclide } & \multicolumn{6}{|c|}{ Release, $\mathbf{C}^{\mathbf{a}}$} \\
\hline & 100 Areas & $\begin{array}{l}\text { 200 Eust } \\
\text { Area }\end{array}$ & $\begin{array}{l}200 \text { West } \\
\text { Area }\end{array}$ & 300 Area & 400 Area $^{t}$ & Total \\
\hline${ }^{3} \mathrm{H}$ (as HTO) & $\mathrm{NM}$ & NM & NM & 2.80 & 2.5 E-02 & 2.83 \\
\hline${ }^{3} \mathrm{H}($ as HT) & $\mathrm{NM}$ & NM & $\mathrm{NM}$ & 3.84 & NM & 3.84 \\
\hline${ }^{60} \mathrm{Co}$ & $9.2 \mathrm{E}-06$ & NM & NM & ND & $\mathrm{NM}$ & $9.2 \mathrm{E}-06$ \\
\hline${ }^{65} \mathrm{Zn}$ & ND & $\mathrm{NM}$ & NM & ND & $\mathrm{NM}$ & ND \\
\hline${ }^{90} \mathrm{Sr}$ & $6.1 \mathrm{E}-05$ & $6.8 \mathrm{E}-05^{\mathrm{b}}$ & $1.0 \mathrm{E}-04^{\mathrm{b}}$ & $1.8 \mathrm{E}-05^{\mathrm{b}}$ & $6.1 \mathrm{E}-08^{\mathrm{b}}$ & $2.8 \mathrm{E}-04^{\mathrm{b}}$ \\
\hline${ }^{9} \mathrm{Zr}$ & ND & $\mathrm{ND}$ & ND & ND & NM & $\mathrm{ND}$ \\
\hline${ }^{106} \mathrm{Ru}$ & 1.1 E-05 & $6.7 \mathrm{E}-06$ & $1.0 \mathrm{E}-08$ & ND & NM & $1.8 \mathrm{E}-05$ \\
\hline${ }^{133} \mathrm{Sn}$ & ND & $8.0 \mathrm{E}-07$ & $1.4 \mathrm{E}-07$ & ND & NM & $9.4 \mathrm{E}-07$ \\
\hline${ }^{125} \mathrm{Sb}$ & $2.8 \mathrm{E}-06$ & $9.1 \mathrm{E}-06$ & $1.2 \mathrm{E}-07$ & ND & NM & $1.2 \mathrm{E}-05$ \\
\hline${ }^{129} \mathrm{I}$ & NM & $8.9 \mathrm{E}-03$ & $\mathrm{NM}$ & ND & $\mathrm{NM}$ & $8.7 \mathrm{E}-03$ \\
\hline${ }^{131} I$ & $\mathrm{NM}$ & ND & ND & ND & ND & ND \\
\hline${ }^{134} \mathrm{Cs}$ & $1.3 \mathrm{E}-06$ & $2.4 \mathrm{E}-08$ & $1.1 \mathrm{E}-07$ & $2.7 \mathrm{E}-08$ & NM & $1.5 \mathrm{E}-06$ \\
\hline${ }^{137} \mathrm{Cs}$ & $2.5 \mathrm{E}-04$ & $3.8 \mathrm{E}-04$ & $1.9 \mathrm{E}-05$ & $1.5 \mathrm{E}-06$ & $5.9 \mathrm{E}-06^{\mathrm{c}}$ & $6.6 \mathrm{E}-04^{\mathrm{c}}$ \\
\hline${ }^{152} \mathrm{Eu}$ & ND & $3.7 \mathrm{E}-07$ & $1.6 \mathrm{E}-07$ & $\mathrm{ND}$ & NM & $5.2 \mathrm{E}-07$ \\
\hline${ }^{154} \mathrm{Eu}$ & 8.3 E-06 & $4.6 \mathrm{E}-07$ & $2.6 \mathrm{E}-07$ & ND & $\mathrm{NM}$ & 9.1 E-06 \\
\hline${ }^{155} \mathrm{Eu}$ & $1.5 \mathrm{E}-06$ & $2.2 \mathrm{E}-07$ & 1.2 E-07 & $4.3 \mathrm{E}-08$ & NM & $1.8 \mathrm{E}-06$ \\
\hline${ }^{220} \mathrm{Rn}$ & NM & $\mathrm{NM}$ & $\mathrm{NM}$ & 79.0 & NM & 79.0 \\
\hline${ }^{222} \mathrm{Rn}$ & NM & NM & $\mathrm{NM}$ & 0.4 & NM & 0.4 \\
\hline Uranium, depleted $^{d}$ & NM & NM & NM & $2.2 \mathrm{E}-08$ & NM & $2.2 \mathrm{E}-08$ \\
\hline${ }^{238} \mathrm{Pu}$ & $2.3 \mathrm{E}-06$ & $6.9 \mathrm{E}-07$ & $2.6 \mathrm{E}-06$ & $2.5 \mathrm{E}-09$ & NM & $5.6 \mathrm{E}-06$ \\
\hline $239.240 \mathrm{Pu}$ & $1.5 \mathrm{E}-05$ & $7.9 \mathrm{E}-06^{\circ}$ & $1.0 \mathrm{E}-04^{\mathrm{e}}$ & $2.1 \mathrm{E}-06^{\mathrm{e}}$ & $1.7 \mathrm{E}-06^{\mathrm{e}}$ & $1.3 \mathrm{E}-04^{\mathrm{e}}$ \\
\hline${ }^{24} \cdot \mathrm{Pu}$ & $2.1 \mathrm{E}-04$ & $1.2 \mathrm{E}-04$ & $2.0 \mathrm{E}-04$ & NM & $\mathrm{NM}$ & $5.3 \mathrm{E}-04$ \\
\hline${ }^{241} \mathrm{Am}$ & $5.7 \mathrm{E}-06$ & $1.6 \mathrm{E}-05$ & $1.7 \mathrm{E}-05$ & $1.1 \mathrm{E}-08$ & $\mathrm{NM}$ & 3.9 E-05 \\
\hline
\end{tabular}

Notes:

a $1 \mathrm{Ci}=3.7 \mathrm{E}+10$ Becquerel; $\mathrm{ND}=$ not detected (i.e, either the radionuclide was not detected in any sample during the year, or the average of all the measurements for that given radionuclide or type of radioactivity made during the year was below background levels); $N M=$ not measured.

b This value includes total beta release data. Total beta and unspecified beta results assumed to be ${ }^{90} \mathrm{Sr}$ for dose calculations.

c This value includes total beta release data. Total beta results assumed to be ${ }^{139} \mathrm{Cs}$ for dose calculations from FFTF emissions.

d Determmed from total alpha measurements. Assumed to be depleted tranium consisting of $63.478 \mathrm{Ci} \%{ }^{138} \mathrm{U}, 0.821 \mathrm{Ci} \%$ ${ }^{235} \mathrm{U}$, and $35.701 \mathrm{Ci} \%{ }^{234} \mathrm{U}\left(99.797 \mathrm{Wt} \%{ }^{238} \mathrm{U}, 0.200 \mathrm{Wt} \%{ }^{235} \mathrm{U}\right.$, and $\left.0.003 \mathrm{Wt} \%{ }^{234} \mathrm{U}\right)$.

e This value includes total alpha release data. Total alpha and unspecified alpha results assumed to be ${ }^{239 / 240} \mathrm{Pu}$ for dose calculations.

f The 400 Area releases contain releases from the EP- $6652 \mathrm{H}-01-\mathrm{V}$ stack, located in the 600 Area on the Fitzner-Eberhardt Arid Lands Ecology Reserve. 
Table 3-2

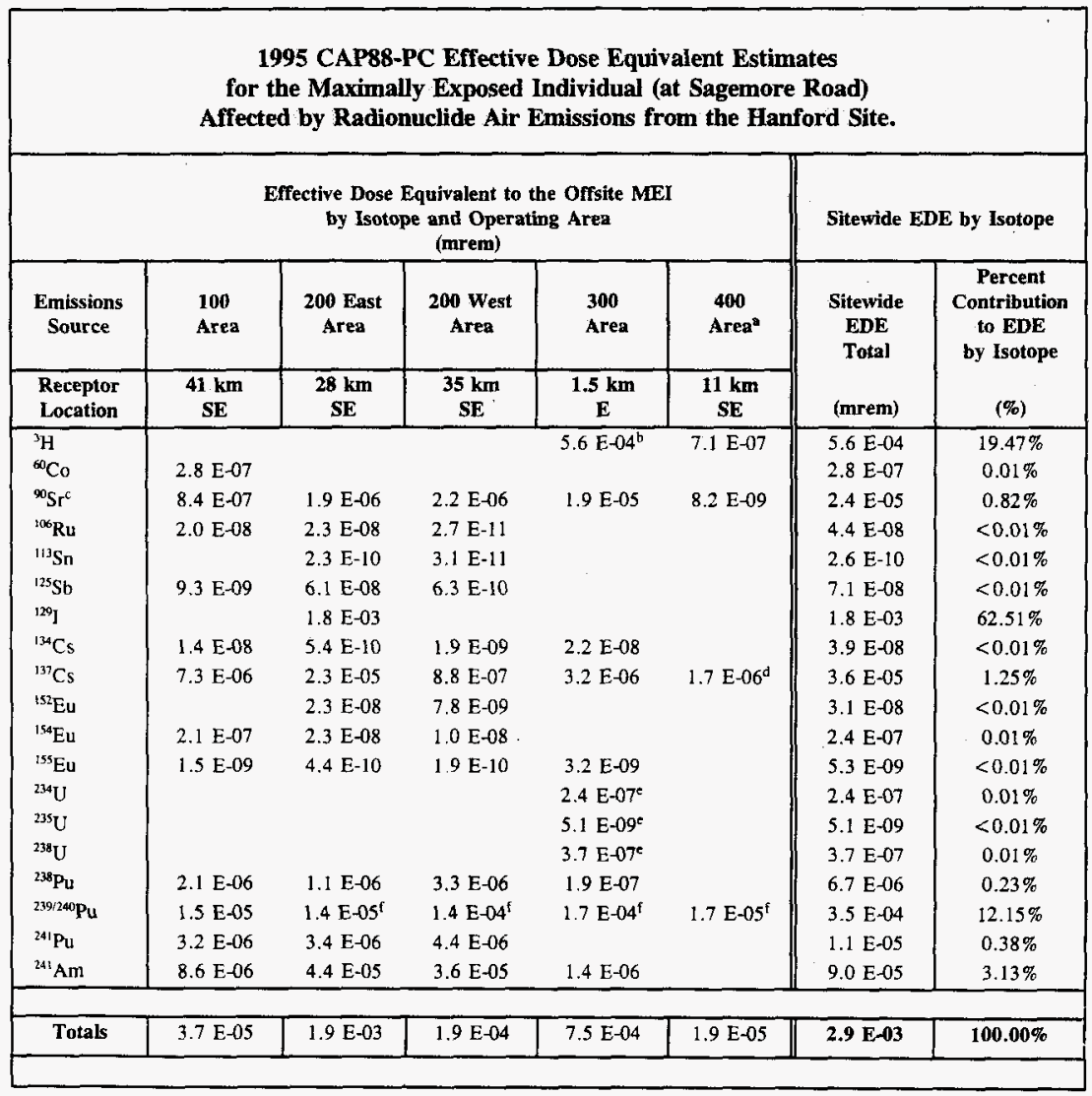

a The 400 Area totals contain releases from the EP-6652H-01-V stack, located in the 600 Area on the Fitzner-Eberhardt Arid Lands Ecology Reserve.

b The HT retease was converted to equivalent curies of HTO for dose modelling purposes because the CAP- 88 models assurne that tritium is in the oxidized form. For this assessment $1 \mathrm{Ci}$ of $\mathrm{HT}$ was assumed to be equivalent to $0.01 \mathrm{Ci}$ of HTO (Brown 1990).

c This value includes total beta release data. Total beta and unspecified beta results were assumed to be ${ }^{x} \mathrm{Sr}$ for dose calculations at all facilities other than FFTF.

d This value includes total beta release data. Total beta results were assumed to be ${ }^{137} \mathrm{Cs}$ for dose calculations from FFTF emissions.

e Uranium isotope emissions were determined from total alpha measurements, and were assumed to consist of depleted uranium containing $63.478 \%{ }^{278} \mathrm{U}, 0.821 \%{ }^{235} \mathrm{U}$, and $35.701 \%{ }^{234} \mathrm{U}$ by activity $\left(99.797 \mathrm{Wt} \%{ }^{238} \mathrm{U}, 0.200 \mathrm{Wt}^{235} \mathrm{U}\right.$, and $\left.0.003 \mathrm{Wt}_{\mathrm{t}}{ }^{234} \mathrm{U}\right)$.

f This value includes total alpha release data. Total alpha and unspecified alpha results were assumed to be ${ }^{239 / 240} \mathrm{Pu}$ for dose calculations at all facilities other than those where the emissions were known to consist of depleted uranium (see footnote e). 


\subsection{METEOROLOGICAL DATA}

Radionuclide air emissions disperse once they enter the atmosphere. Atmospheric dispersion models predict the degree of dilution and the magnitude of resulting air concentrations. Site-specific measurements of the occurrence frequencies for wind speed, wind direction, and atmospheric stability are used in the models. The dispersion models yield annual average dispersion factors (in units of $s / \mathrm{m}^{3}$ ). Combining these factors with annual average release rates predicts average radionuclide air concentrations for the year. Annual average dispersion factors around the 100, 200,300, and 400 Areas for 1995 are in Appendix A.

\subsection{UNPLANNED RELEASES OF RADIONUCLIDES TO THE ATMOSPHERE}

During 1995, no unplanned releases of radionuclides to the atmosphere occurred from facilities at the Hanford Site. Releases from point sources on the Hanford Site were monitored. The resulting quantities of radionuclides released and the corresponding EDEs are reported in Sections 2.0 and 3.0 of this document.

\subsection{ADDITIONAL INFORMATION}

\subsubsection{Projects Requiring Approval or Waiver Under 40 CFR 61}

No waivers of the approval process under 40 CFR 61.96 were exercised in 1995 . In late 1992, the Environmental Protection Agency (EPA) determined that the Hanford Site was not in compliance with 40 CFR 61 , Subpart H. Consequently, EPA approval was requested for all construction or modification projects with the potential to increase radionuclide air emissions.

\subsubsection{Doses to the MEI due to Radon and Thoron Emissions}

The dose to the MEI due to ${ }^{220} \mathrm{Rn}$ (thoron) and ${ }^{222} \mathrm{Rn}$ (radon) emissions, which are not regulated under 40 CFR Part 61 Subpart $H$, are included in Table 3-3. Releases of Rn-220 were modeled by assuming that the short-lived gas had decayed to the next long-lived member of the decay chain, $\mathrm{Pb}-212$, prior to transport offsite. These emissions result in a significant portion of the dose received by the MEI, but are specifically excluded from 40 CFR 61 Subpart $H$ (NESHAP) regulations. Sections 5.2, 5.3, and 5.4 contain additional information on radon and thoron emissions. 
Table 3-3

\begin{tabular}{|c|c|c|}
\hline \multicolumn{2}{|c|}{$\begin{array}{c}\text { 1995 Effective Dose Equivalent to MEI } \\
\text { (at Sagemore Road) from } 300 \text { Area } \\
\text { Radon and Thoron Emissions. }\end{array}$} \\
\hline \multicolumn{2}{|c|}{ Isotope } & $\begin{array}{c}\text { Fraction of the } \\
\text { Sitewide EDE, } \\
\text { including Rn } \\
(\%)\end{array}$ \\
\hline${ }^{220} \mathrm{Rn}$ & $(\mathrm{mrem})$ & $51.80 \%$ \\
\hline${ }^{222} \mathrm{Rn}$ & $3.3 \mathrm{E}-03$ & $2.98 \%$ \\
\hline
\end{tabular}

\subsubsection{Supplementary Data from Dose Modelling Results}

Unit dose factors, organized by operating area and radionuclide, were calculated from the releases and dose modelling results. These factors were then used to calculate the point source and radionuclide specific doses included in Table 2-1. Table 3-4 contains the EDE to the MEI and percentage of the sitewide dose, displayed by major emission unit. 
Table 3-4

\begin{tabular}{|c|c|c|}
\hline \multicolumn{3}{|c|}{$\begin{array}{c}\text { Total Doses from Individual Major Point Sources } \\
\text { at the Hanford Site in } 1995 .\end{array}$} \\
\hline $\begin{array}{l}\text { Source Identification } \\
\text { (contractor) }\end{array}$ & $\begin{array}{c}\text { Effective } \\
\text { Dose Equivalent } \\
\text { (mrem) }\end{array}$ & $\begin{array}{c}\text { Percent of Hanford } \\
\text { Site-Related Offsite Dose } \\
(\%)\end{array}$ \\
\hline \multicolumn{3}{|c|}{200 East Area Point Sources } \\
\hline 291-A-1 (WHC) & $1.9 \overline{\mathrm{E}}-\overline{03}$ & $64.7 \%$ \\
\hline 296-A-1 (WHC) & $5 . \overline{\mathrm{E}}-06$ & $0.2 \%$ \\
\hline 291-B-1 (WHC) & $1.5 \mathrm{E}-05$ & $0.5 \%$ \\
\hline 296-A-22 (WHC) & $7.7 \mathrm{E}-07$ & $<0.1 \%$ \\
\hline 296-A-12(WHC) & \multicolumn{2}{|c|}{ (did not operate) } \\
\hline 296-A-17/296-P-26 (WHC) & $6.0 \mathrm{E}-05$ & $2.1 \%$ \\
\hline 296-A-25 (WHC) & $8.7 \mathrm{E}-08$ & $<0.1 \%$ \\
\hline 296-A-40 (WHC) & $3.3 \mathrm{E}-06$ & $0.1 \%$ \\
\hline 296-B-28 (WHC) & $1.0 \mathrm{E}-09$ & $<0.1 \%$ \\
\hline $296-C-5$ (WHC) & $3.8 \mathrm{E}-08$ & $<0.1 \%$ \\
\hline 296-P-16 (WHC) & $1.2 \mathrm{E}-07$ & $<0.1 \%$ \\
\hline 296-P-32, 33, and 34 (WHC) & $4.1 \bar{E}-09$ & $<0.1 \%$ \\
\hline \multicolumn{3}{|c|}{200 West Area Point Sources } \\
\hline 291-Z-1 (WHC) & $1.5 \mathrm{E}-04$ & $5.0 \%$ \\
\hline 296-S-15 (WHC) & $1.6 \overline{\mathrm{E}}-08$ & $<0.1 \%$ \\
\hline 296-S-22 (WHC) & $1.6 \mathrm{E}-09$ & $<0.1 \%$ \\
\hline 296-T-18 (WHC) & $2.7 \mathrm{E}-09$ & $<0.1 \%$ \\
\hline \multicolumn{3}{|c|}{300 Area Point Sources } \\
\hline 340-NT-EX (WHC) & $5.6 \mathrm{E}-06$ & $0.2 \%$ \\
\hline EP.324-01-S (PNNL) & $2.9 \mathrm{E}-04$ & $10.1 \%$ \\
\hline EP-325-01-S (PNNL) & $2.7 \mathrm{E}-04$ & $9.4 \%$ \\
\hline EP-327-01-S (PNNL) & $3.9 \mathrm{E}-05$ & $1.4 \%$ \\
\hline EP-327-02-V (PNNL) & $8.5 \mathrm{E}-08$ & $<0.1 \%$ \\
\hline EP-3720-01-S (PNNL) & $2.5 \mathrm{E}-06$ & $0.1 \%$ \\
\hline Major Point Sources Totals & $2.7 \mathrm{E}-03$ & $95.2 \%$ \\
\hline Minor Point Sources Totals & $1.4 \mathrm{E}-04$ & $4.8 \%$ \\
\hline All Point Sources Totaled & $2.9 \mathrm{E}-03$ & 100.0 \\
\hline
\end{tabular}

Note:

a $1 \mathrm{mrem}=1.0 \mathrm{E}-02 \mathrm{mSv}$; Doses and percentages in this table exclude those attributable to radon and thoron emissions. 


\subsection{CERTIFICATION}

This certification applies to Sections $1.0,2.0$, and 3.0 only.

"I certify under penalty of law that I have personally examined and am familiar with the information submitted herein and, based on my inquiry of those individuals immediately responsible for obtaining the information, I believe that the submitted information is true, accurate, and complete. I am aware that there are significant penalties for submitting false information, including the possibility of fine and imprisonment. See, 18 U.S.C. 1001."
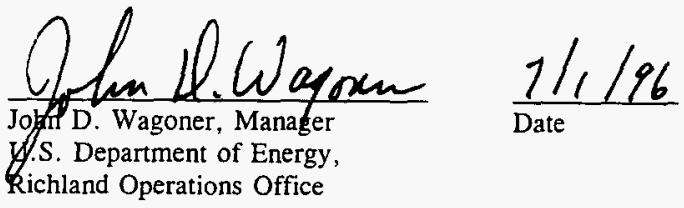
DOE/RL-96-37

This page intentionally left blank. 


\subsection{DIFFUSE AND FUGITIVE SOURCES OF EMISSIONS}

The Washington Administrative Code, WAC 246-247, requires facilities to monitor nonpoint (diffuse) and fugitive emissions of radioactive material. DOE Headquarters has requested that estimated doses to the public, related to radioactive emissions from both point sources along with diffuse and fugitive sources, be included in this report. This request is based on the requirement, in 40 CFR 61 Subpart $H$, that the dose from all potential emission sources at DOE facilities shall not exceed $10 \mathrm{mrem} / \mathrm{y}$ EDE to any member of the public. This section describes Hanford's diffuse and fugitive emissions monitoring program and estimates the effective dose equivalent (EDE) to the public, attributable to diffuse and fugitive sources.

Currently, all nuclear material production facilities at the Hanford Site are shutdown or in standby mode. Only waste minimization and stabilization processes continue to operate. In the past, when the Hanford Site was operating at or near full capacity, point source emissions were easily detected. Now, however, emissions from point sources have diminished in most instances to background levels. Therefore, the environmental contribution from diffuse and fugitive emissions has become a larger percentage of the total radioactivity attributable to activities at the Hanford Site, even though diffuse and fugitive emissions have not increased.

Methodologies for caiculating doses from point sources are well established, based primarily on analyzing samples and measuring flow of stack emissions. Similar methodologies are still being developed for diffuse and fugitive sources because of complicating factors such as: (1) difficulty in accurately quantifying air flow from the source, (2) more complex influences from meteorological conditions, and (3) exceedingly low detection levels.

For this report, doses have been calculated for emissions from both point and diffuse and fugitive sources, even though the methods used for calculating diffuse and fugitive emissions are still being refined. Dose calculations for each type of emission are provided separately. As methods for estimating the dose from diffuse and fugitive emissions become more refined, more precise amounts representing radionuclides occurring naturally, from nuclear-testing fallout, and non-DOE nuclear facilities (see Figure 4-1) may be subtracted from ambient air concentrations. Undoubtedly this would lower dose estimates attributable to the Hanford Site.

\subsection{DIFFUSE AND FUGITIVE EMISSIONS MONITORING}

At Hanford two programs monitor diffuse and fugitive emissions, the Near-Facility Environmental Monitoring Program and the Environmental Surveillance Program. These 
Figure 4-1. Monthly Average Gross Beta Radioactivity in Airborne Particulate Samples, 1981 through 1991 (PNL 1990).

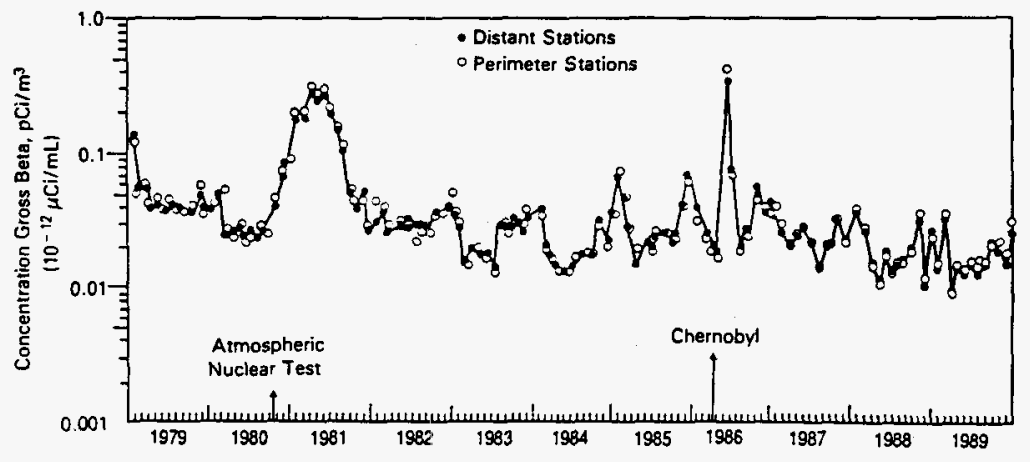

two programs monitor locations on and off of the Hanford Site. The intent of these programs is to detect and quantify radiological and non-radiological contaminants and assess their environmental and human health significance. These programs monitor contaminants released to the environment, which may reach humans via all pathways. This report only presents monitoring information relative to radiological contaminants released to the environment such that they result in airborne releases.

\subsubsection{Near-Facility Environmental Monitoring}

Near-facility environmental monitoring is defined as foutine monitoring near facilities that have discharged, have the potential to discharge, store, or have disposed of radioactive contaminants. The near-facility environmental monitoring at Hanford is performed by WHC. Monitoring locations are associated mostly with major nuclear facilities and waste storage or disposal facilities such as: burial grounds, tank farms, ponds, cribs, trenches, and ditches.

Routine monitoring activities include sampling and monitoring ambient air, surface contamination, water from surface-water disposal units, external radiation doses, vadose zone, soil, sediment, vegetation, and animals. Samples are collected from known or 
expected effluent pathways, which are generally downwind of potential or actual airborne releases and downgradient of liquid discharges. Ambient air monitoring is the primary method utilized in monitoring for diffuse and fugitive emissions. Other monitoring and sampling methods (e.g. surface contamination surveys, soil sampling, etc...) are utilized as secondary indicators of diffuse and fugitive emissions.

Radioactivity in air is sampled by a network of continuously operating samplers at 47 locations near facilities: four are located in the 100-N Area, four are located in the 100-K Area, 37 are located in the 200 Areas, one is located near the 300 Area Treated Effluent Disposal Facility, and one station at the Wye Barricade which is collocated with samplers operated by the PNNL Surface Environmental Surveillance Project and the Washington State Department of Health (WDOH). Ambient air samplers are primarily located at or near sites and facilities (within $500 \mathrm{~m}$ [1500 ft]) having the potential for or history of environmental releases. All ambient air samples are analyzed for total alpha, total beta, gamma emitters, ${ }^{90} \mathrm{Sr},{ }^{234} \mathrm{U},{ }^{235} \mathrm{U},{ }^{238} \mathrm{U},{ }^{238} \mathrm{Pu}$, and ${ }^{239 / 240} \mathrm{Pu}$. Gamma emitters routinely reported include ${ }^{40} \mathrm{~K}$, ${ }^{60} \mathrm{Co},{ }^{65} \mathrm{Zn},{ }^{95} \mathrm{Zr},{ }^{106} \mathrm{Ru},{ }^{125} \mathrm{Sb},{ }^{134} \mathrm{Cs},{ }^{137} \mathrm{Cs},{ }^{144} \mathrm{Ce},{ }^{154} \mathrm{Eu},{ }^{155} \mathrm{Eu}$, and any other detectable gamma emitters. The $100-\mathrm{K}$ Area air samples are also analyzed for ${ }^{241} \mathrm{Am}$ and ${ }^{241} \mathrm{Pu}$. Figure 4-2 depicts the locations of the ambient air samplers utilized for near-facility monitoring.

More detailed descriptions of this program can be found in the Westinghouse Hanford Company Operational Environmental Monitoring Annual Report (WHC 1996) and the Hanford Site Environmental Report (PNNL 1996a).

\subsubsection{Environmental Surveillance}

Environmental Surveillance encompasses sampling and analyzing for potential radiological contaminants on and off the Hanford Site. Envirormental surveillance at Hanford is performed by PNNL through the Surface Environmental Surveillance Project. Monitoring locations are divided into three surveillance zones located on and off of the Hanford Site.

The first surveillance zone extends from the near-facility monitoring locations to the Site perimeter. The second surveillance zone consists of a series of perimeter sampling stations near or just inside the Site boundary and along State Highway 240 . The third surveillance zone consists of nearby and distant sampling locations within an $80 \mathrm{~km}(50 \mathrm{mi})$ radius of the Site. Background sampling is performed at distant locations, such that the measurements are essentially unaffected by Hanford operations.

Routine surveillance activities include air, surface-water, ground-water, food and farm products, fish and wildlife, soil and vegetation, and external radiation sampling and monitoring. Like the near-facility monitoring program, ambient air sampling is the primary method utilized in monitoring diffuse and fugitive emissions. 
Figure 4-2. Near-Facility Ambient Air Sampling Locations.

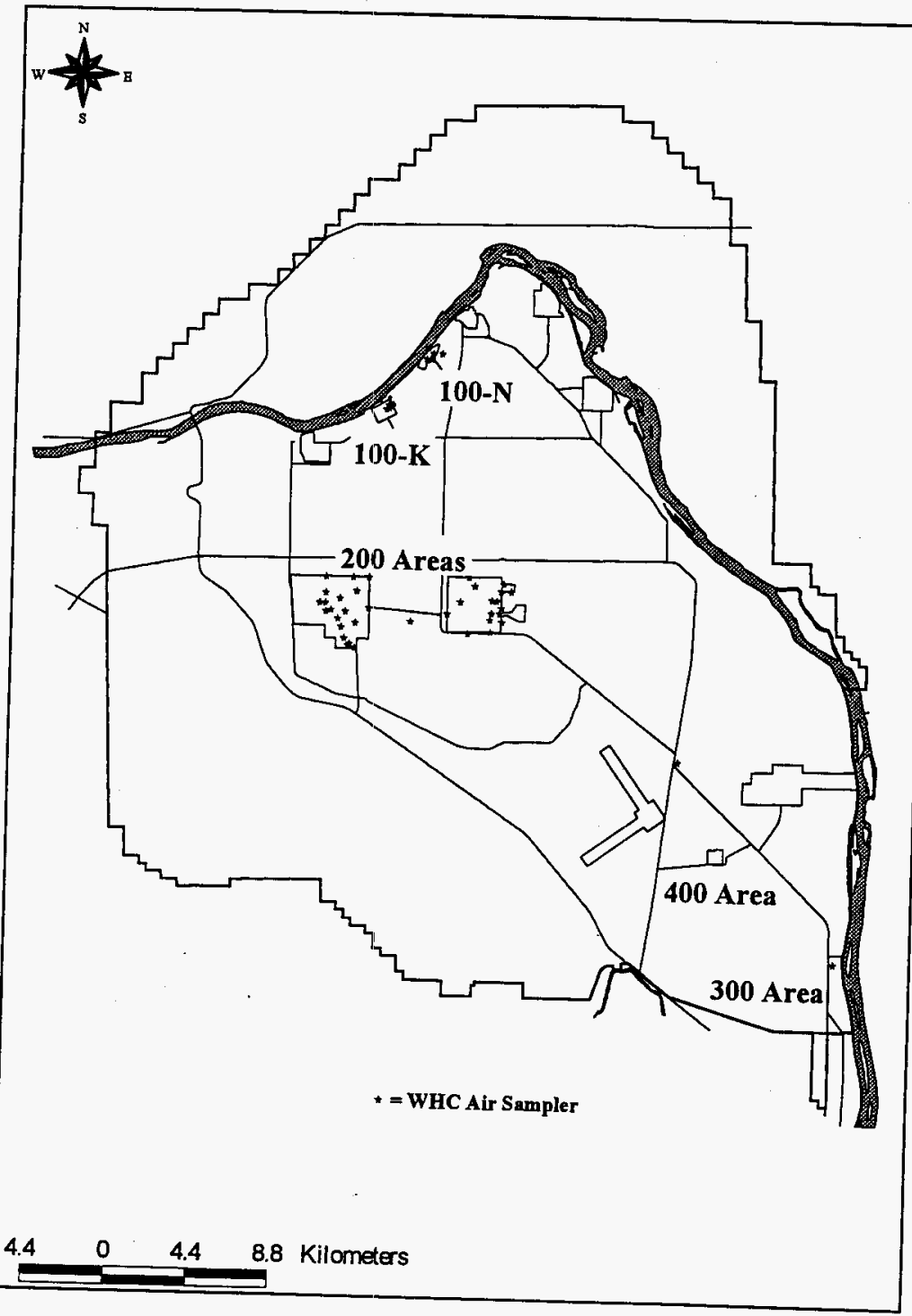


The air surveillance network consists of 40 sampling stations, of which 20 are located on the site (zone 1), 9 are located at the site perimeter (zone 2), 8 are in nearby communities (zone 3 ), and 3 are in distant communities (background locations). This program routinely monitors for radioactive vapors, gases, and aerosols. Vapor, gas, and liquid aerosol sampling and analysis is performed for ${ }^{3} \mathrm{H},{ }^{129} \mathrm{I}$, and ${ }^{131} \mathrm{I}$, at selected locations. Solid aerosols (particulate) are sampled and analyzed for at all sampling locations. Particulate air samples are routinely analyzed for total alpha, total beta, gamma emitters, ${ }^{90} \mathrm{Sr},{ }^{234} \mathrm{U},{ }^{235} \mathrm{U},{ }^{238} \mathrm{U}$, ${ }^{238} \mathrm{Pu}$, and ${ }^{239 / 240} \mathrm{Pu}$. Figure 4-3 depicts the locations of the Surface Environmental Surveillance Project's ambient air samplers utilized for air monitoring.

A more detailed description of this program can be found in the Hanford Site Environmental Report (PNNL 1996a).

\subsection{ESTIMATED DOSES FROM DIFFUSE AND FUGITIVE EMISSIONS}

The potential dose to an offsite individual and the potential releases from diffuse and fugitive sources were estimated using ambient air monitoring data from environmental surveillance air sampling locations along the perimeter of the Hanford Site. Data from six perimeter locations were used to perform the assessment of the 1995 emissions, see Figure 4-3. These stations were located downwind of the Hanford Site (northeast to south) (PNNL 1996a, PNNL 1996b).

\subsubsection{Description of Dose Assessment Method}

Hanford has elected to use environmental surveillance ambient air monitoring data collected at the site perimeter to estimate the dose from diffuse and fugitive emissions sources. This method is preferred over other possible methods for two reasons: 1) this data most accurately represents the actual exposures of an offsite individual to airborne radioactivity, and 2) there is currently insufficient information about the extent and characteristics of soil contamination on the Hanford Site to use resuspension estimates in conjunction with dispersion models. The ambient air sampling results consisted of measured air concentrations for radionuclides that may be released from site operations and diffuse and fugitive sources. Radionuclides sampled and analyzed for include: ${ }^{3} \mathrm{H},{ }^{60} \mathrm{Co},{ }^{90} \mathrm{Sr},{ }^{129} \mathrm{I},{ }^{131} \mathrm{I}$. ${ }^{134} \mathrm{Cs},{ }^{137} \mathrm{Cs},{ }^{154} \mathrm{Eu},{ }^{155} \mathrm{Eu},{ }^{238} \mathrm{Pu},{ }^{239 / 240} \mathrm{Pu},{ }^{241} \mathrm{Am}$, and total uranium. Particulate samples were also analyzed for naturally occurring radionuclides $\mathrm{Be}-7$ and $\mathrm{K}-40$. However, they were not included in the dose assessment, because the background levels of these radionuclides are relatively high and there is no known potential for contribution from onsite sources.

Radionuclide air concentrations resulting from 1995 stack emissions were calculated for each of the six selected perimeter sample locations using the CAP88-PC atmospheric dispersion modelling code. The combined contributions to airborne radionuclide 
Figure 4-3. Environmental Surveillance Ambient Air Sampling Locations.

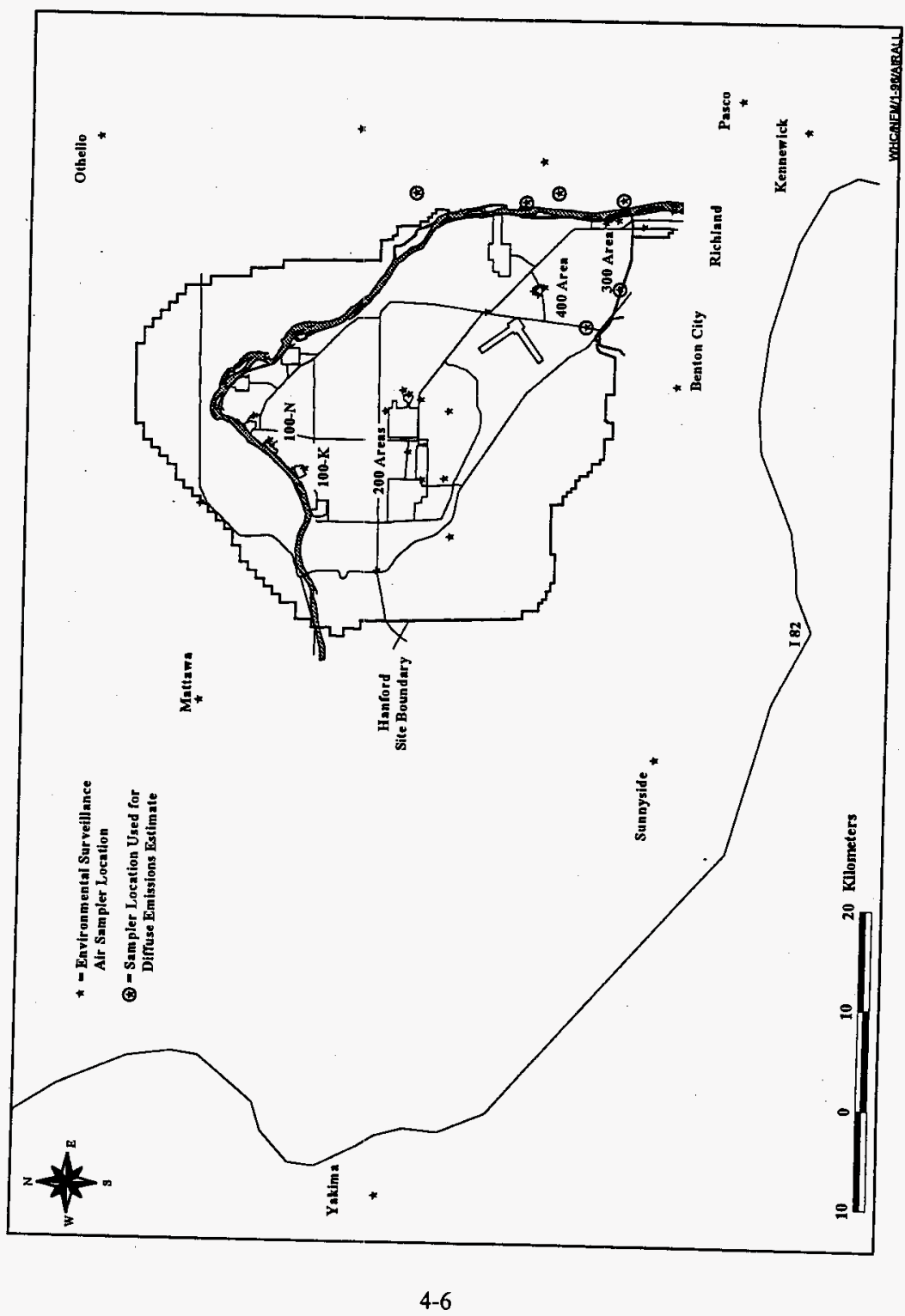


concentrations attributable to the stack emissions from all operating areas were subtracted from the ambient air sampling results. Averaged regional background concentrations for each radionuclide were calculated from the air sample results obtained from distant community sampling stations, in Yakima, Toppenish, and Sunnyside. The average background concentration at these stations was also subtracted from the ambient monitoring results at perimeter stations, leaving the radionuclide concentrations attributable to diffuse and fugitive emissions. Note that the radionuclide concentrations attributable to emissions from non-DOE nuclear sites have not been subtracted, since this data is usually not available at the time this report is prepared. As a result the actual diffuse and fugitive emissions from the Hanford Site are likely to be somewhat lower than the estimates presented in this report.

Releases from diffuse and fugitive sources were estimated using the average air concentrations, attributable to diffuse and fugitive emissions, and by performing a backcalculation using CAP88-PC. The 200 West Area was assumed to be the source of all diffuse and fugitive emissions, resulting in the highest release estimates.

The estimated diffuse and fugitive emissions were then used to calculate doses at selected Site perimeter locations with the CAP88-PC code. All diffuse and fugitive emissions were modelled from the 200 West Area

\subsubsection{Results of Dose Assessment}

During 1995, the annual average ambient air concentrations at downwind perimeter sampling stations were found to be numerically greater than the combined contributions from stack releases and background for several analytes, including ${ }^{3} \mathrm{H}$, total uranium, ${ }^{238} \mathrm{Pu}-238$, ${ }^{239} \mathrm{Pu}$ and ${ }^{241} \mathrm{Am}$. However, these perimeter concentrations were not statistically different from those measured at the distant community stations (background locations), which are unaffected by Hanford effluents (PNNL 1996a, PNNL 1996b). Concentrations of other radionuclides may have been greater than those caused by stack releases and background radioactivity, at individual monitoring stations. However, the estimated releases based on the corrected average air concentrations, for those nuclides at all sampling stations, was less than zero.

Releases from diffuse and fugitive sources were estimated from the corrected air concentrations by performing a back-calculation using CAP88-PC. The 200 West Area was assumed to be the source of diffuse and fugitive emissions for all radionuclides, which results in the highest release estimate. The diffuse and fugitive source releases reported in Table 41 represent the mean of the release estimates, for each of the selected downwind perimeter locations at which a particular radionuclide was sampled. Note that not all radionuclides were evaluated at every sampling station; the estimated releases for any particular radionuclide are based on those stations where samples were analyzed for that radionuclide. 
Table 4-1

\begin{tabular}{|c|c|c|c|}
\hline \multicolumn{4}{|c|}{$\begin{array}{c}\text { Estimated } 1995 \text { Hanford Site Diffuse and Fugitive Emissions } \\
\text { and Resulting Effective Dose Equivalent". }\end{array}$} \\
\hline \multicolumn{2}{|l|}{ Location - } & $\begin{array}{l}\text { Byers Landing } \\
\text { (closest to } \\
\text { MEI Location) }\end{array}$ & $\begin{array}{l}\text { Prosser Barricade } \\
\text { (highest dose from } \\
\text { emissions) }\end{array}$ \\
\hline Radionuclide $\mathbf{V}$ & $\begin{array}{c}\text { Diffuse \& Fugitive Source } \\
\text { Estimated Release from } \\
200 \mathrm{Areas} \\
(\mathrm{Ci} / \mathrm{y})^{\mathbf{b}}\end{array}$ & $\begin{array}{l}\text { Estimated Dose } \\
\text { at Location } \\
\text { (mrem/y) }\end{array}$ & $\begin{array}{l}\text { Estimated Dose } \\
\text { at Location } \\
(\operatorname{mrem} / y)^{c}\end{array}$ \\
\hline $\begin{array}{l}{ }^{3 \mathrm{H}} \\
{ }^{60} \mathrm{Co} \\
{ }^{90} \mathrm{Sr} \\
{ }^{129} \mathrm{I} \\
{ }^{131} \mathrm{I} \\
{ }^{134} \mathrm{Cs} \\
{ }^{137} \mathrm{Cs} \\
{ }^{154} \mathrm{Eu} \\
{ }^{155} \mathrm{Eu} \\
\text { total uranium } \\
{ }^{238} \mathrm{Pu} \\
{ }^{239.240} \mathrm{Pu} \\
{ }^{241} \mathrm{Am} \\
\end{array}$ & $\begin{array}{l}7.4 \mathrm{E}+02 \\
-1.2 \mathrm{E}-01 \\
-1.2 \mathrm{E}-03 \\
-5.1 \mathrm{E}-02 \\
-3.5 \mathrm{E}+02 \\
-1.7 \mathrm{E}-01 \\
-2.5 \mathrm{E}-02 \\
-1.0 \mathrm{E}+00 \\
-5.6 \mathrm{E}-02 \\
7.1 \mathrm{E}-03 \\
7.7 \mathrm{E}-05 \\
1.2 \mathrm{E}-04 \\
2.0 \mathrm{E}-04 \\
\end{array}$ & $\begin{array}{l}7.6 \mathrm{E}-03 \\
2.0 \mathrm{E}-04 \\
3.4 \mathrm{E}-04 \\
8.9 \mathrm{E}-04 \\
\end{array}$ & $\begin{array}{l}1.4 \mathrm{E}-02 \\
3.7 \mathrm{E}-04 \\
6.0 \mathrm{E}-04 \\
1.6 \mathrm{E}-03 \\
\end{array}$ \\
\hline & Total & $1.8 \mathrm{E}-02$ & 3.0 E-02 \\
\hline
\end{tabular}

Notes:

a Releases from non-DOE nuclear facilities have not been subtracted from these diffuse and fugitive emissions estimates. Only Hanford stack emissions and background radioactivity have been subtracted from these diffuse and fugitive emissions estimates.

Diffuse and fugitive source releases are assumed to originate at the 200 West Area with a release height of $1 \mathrm{~m}$. Negative values for releases of a particular radionuclide indicate that air concentrations at the site perimeter are lower than the combined air concentrations expected from natural background and monirored stack releases. Therefore, radionuclides for which the average release estimate from diffuse and fugitive sources was negative were assumed to make no contribution to the offsite dose.

b $1 \mathrm{Ci}=3.7 \mathrm{E}+10$ Becquerel; Emissions from diffuse and fugitive sources are assumed to originate in the Hanford Site 200 Areas. The 300 Area also has potential sources for resuspension of uranium from soil, in addition to naturally occurring uranium isotopes found throughout the area. Because it was not possible to determine the source of uranium isotopes detected at offsite sample stations, the releases were modeled as if the total inventory were from the 200 Areas.

c 1 mrem $=1.0 \mathrm{E}-02 \mathrm{mSv}$; Dose is based on air monitoring results for sample stations downwind, generally northeast to south, of the Hanford Site. The measured air concentrations for these locations are corrected for estimated contributions from stack releases and regional background levels of monitored radionuclides. Radionuclide emissions which are reported as being less than zero result from air monitoring results that are below the detection limit, or for which the monitoring results are lower than the contributions from stack releases and background. Doses for these radionuclides are assumed to be zero. 
In accordance with previous guidance from Department of Energy headquarters, the average release estimates for diffuse and fugitive sources were calculated using air concentrations that were both positive and negative with respect to the regional background and stack emission estimates. Where the resulting mean release estimate reported in Table 41 for an individual radionuclide is less than zero, it indicates that the average of the monitored air concentrations at the downwind perimeter stations was numerically smaller than the combined concentrations expected as a result of stack emissions and regional background. In such cases, it is unlikely that diffuse and fugitive sources contributed significantly to the offsite measured air concentrations for those radionuclides.

The estimated diffuse fugitive source releases for the sampled radionuclides were also used to calculate the dose at the downwind perimeter sampling stations. The Prosser Barricade station had the highest predicted air concentrations, and the Byers Landing station was closest to the Sagemore Road location, which is the location of the MEI for monitored point sources at the Hanford Site. The 1995 results in Table 4-1 indicate that the hypothetical mean doses to individuals at these two locations were $3.0 \times 10^{-2} \mathrm{mrem} / \mathrm{y}$ and $1.8 \times 10^{-2} \mathrm{mrem} / \mathrm{y}$, respectively. The doses for the other four sampling stations were lower than those at the Prosser Barricade station, but are not shown in Table 4-1.

Where the release estimate for a particular radionuclide was numerically less than zero, the dose estimate for that nuclide was set equal to zero before combining the contributions of all radionuclides to obtain the total dose at each location. Although the estimated dose from diffuse and fugitive sources at the Sagemore Road location is somewhat higher than the dose from point sources, the combined dose from point sources and diffuse and fugitive sources during 1995 was substantially below the $10 \mathrm{mrem} /$ year standard.

In addition to the sitewide diffuse and fugitive emissions estimates, diffuse and fugitive tritium emissions from two sources were estimated for 1995, in order to determine their potential contribution to the public radiological dose. Tritium emissions from the 100 $\mathrm{K}$ Area spent fuel storage basins were estimated at less than $2 \mathrm{Ci}$ /year, and those from the 200 Area tank farms were estimated at less than $6 \mathrm{Ci} /$ year. Emissions from both sources were assumed to be in the form of tritiated water. During 1995, the resulting dose to the offsite maximally exposed individual at Sagemore Road from the estimated tritium emissions was $1.2 \times 10^{-5}$ mrem for the $100 \mathrm{~K}$ basins, and $9.2 \times 10^{-5}$ mrem for the 200 Area tank farms. The dose from these sources was much lower than the dose from monitored point source emissions and did not substantially increase the total dose to an offsite receptor.

\subsubsection{Estimate of Uncertainty in Dose Assessment}

In order to obtain an estimate of the uncertainty in the diffuse and fugitive emissions' dose estimates, at the Hanford Site perimeter, the reported 1995 air concentrations at the Byers Landing sampling station and at the distant community stations were used to estimate the mean and $95 \%$ confidence intervals for each location. This analysis employed the uncorrected air concentrations at the reference locations, including the contributions from monitored point source releases at DOE facilities, diffuse and fugitive sources, sources other 
than DOE facilities, and regional background. The Byers Landing station was selected for this analysis because it is closest to the location of the Hanford Site MEI, at Sagemore Road, and also was the only perimeter station at which all radionuclides were evaluated.

The calculation was performed using the GENII-S computer code (SNL 1992) to produce a stochastic analysis of the environmental radiation doses. The raw values of the measured air concentrations were input as basic concentrations to define an empirical distribution for each radionuclide. The code then used a Latin hypercube sampling routine to select random values for each radionuclide concentration in 100 trials to obtain the dose distribution for each location. The values of parameters other than the radionuclide air concentrations were not varied as part of this analysis; therefore, the uncertainties reported in this section reflect only variability in the air sampling data.

The estimated mean dose at the Byers Landing station was $6.9 \times 10^{-2} \mathrm{mrem} / \mathrm{y}$ for artificially produced radionuclides sampled at that location, with a $95 \%$ confidence interval of $1.1 \times 10^{-2}$ to $1.4 \times 10^{-1} \mathrm{mrem} / \mathrm{y}$. The corresponding result for the distant community monitoring stations was $5.0 \times 10^{-2} \mathrm{mrem} / \mathrm{y}$ with $95 \%$ confidence limits of 0.0 to $9.8 \times 10^{-2}$ mrem/y. The mean dose estimates and the upper $95 \%$ confidence limits were similar at the site perimeter and at the distant community stations. Because the estimated dose at perimeter stations was largely a result of background radioactive materials (naturally occurring radionuclides and fallout), the estimated diffuse and fugitive source component was a relatively small fraction of the total offsite dose. The $95 \%$ confidence interval for the dose from diffuse and fugitive sources would be expected to be less than, or equal to, than the overall dose at these locations.

\subsubsection{Discussion}

It should be noted that the release estimates for diffuse and fugitive sources in Table 4-1 were obtained using the CAP88-PC code package, which incorporates a continuous release gaussian plume dispersion model. Releases from diffuse and fugitive sources would be expected to occur primarily under conditions that are very different from the annual average assumptions used in the CAP88-PC model. This is particularly true for resuspension of contaminated soil, and to some extent for emissions from sources such as evaporation ponds, which are a function of the wind speed. Because release rates from such sources are greatest under conditions that favor atmospheric dispersion, use of an annual average continuous release model to back-caiculate the release quantities may introduce a significant bias in these estimates. The dose estimates for sources of this type may also be affected by seasonal variation in the resuspension rates due to prevalence of strong winds during certain seasons of the year. If these episodes occur primarily during times when crop production is minimal, some of the exposure pathways incorporated into the CAP88-PC code (direct deposition on human and animal food crops, for example) would not be applicable. The release and dose estimates reported for diffuse and fugitive sources in this evaluation should therefore be viewed as approximations whose accuracy is limited by a number of factors inherent in the sampling and modeling process. 


\subsection{DIFFUSE AND FUGITIVE EMISSION SOURCES}

The Hanford Site consists of $1,450 \mathrm{~km}^{2}\left(560 \mathrm{mi}^{2}\right)$ of semiarid shrub-steppe land, of which approximately $6 \%$ has been disturbed and actively used. This $6 \%$ of land [about $83 \mathrm{~km}^{2}\left(32 \mathrm{mi}^{2}\right)$, or 8,090 ha $(20,000$ acres $)$ ] is divided into large operational and support areas: the 100, 200 East, 200 West, 300, 400, and 1100 Areas. The 600 Area designates the remaining land outside the operational and support areas. Almost all point, diffuse, and fugitive sources of radionuclide emissions are located in the five operational areas; one minor point source is in the 600 Area. Point source emissions are measured directly or calculated from process knowledge. Diffuse and fugitive sources are measured through a network of monitoring systems extending from the potential and actual sources to far offsite.

The Hanford Site was acquired in 1943 and dedicated to producing plutonium for national defense and managing the resulting production wastes. Restoring the Hanford Site environment is the new mission that has largely supplanted the previous operational objectives. The environmental restoration effort will entail activities such as decontaminating and decommissioning over 100 facilities and cleaning up and restoring about 1,500 waste sites. Until the restoration and cleanup work is completed, radioactive emissions may be released from hundreds of diffuse and fugitive sources, in addition to known point-source stacks.

Besides measuring point source emissions and modeling those emissions to determine public doses, environmental surveillance is conducted. Environmental and food-chain pathways are monitored near facilities emitting radionuclides from either point sources or diffuse and fugitive sources. The environmental pathways for all air emissions from the Hanford Site are monitored using a stratified sampling approach. Samples are collected and radiation is measured according to three surveillance zones. These zones extend from main onsite operating areas to offsite regions (PNNL 1996a).

The first zone begins near the operating facilities and ends at the Hanford Site perimeter. Diffuse and fugitive emissions generally will be most concentrated and easier to detect in this zone before diluting further as they drift offsite.

The second surveillance zone is a series of sampling stations that surround the Hanford Site near its perimeter. Because a person could live as close to the Hanford Site as some of these stations, their data represent the maximum exposures for a member of the public. Therefore, ambient air sampling data from the perimeter locations most closely reflect the actual impacts of radionuclide air emissions from point sources and diffuse and fugitive sources on the Hanford Site.

The third surveillance zone encompasses nearby and distant communities within an $80-\mathrm{km}(50-\mathrm{mi})$ radius of the center of the Hanford Site but beyond its boundaries: Surveillance is conducted in communities to provide measurements at those locations where the most people are potentially exposed. This surveillance ensures that radionuclide levels are well below standards established to protect the public health. Finally, background 
concentrations are measured at distant locations and compared with onsite, perimeter, and community locations to indicate the effects of Hanford Site activities. Background locations are essentially unaffected by Hanford Site emissions but contain similar levels of radioactivity originating naturally and from nuclear-testing fallout.

The goal of environmental surveillance at the Hanford Site is to verify compliance with DOE, EPA, and WDOH radiological dose standards for public protection. This goal is accomplished by measuring radionuclides and consequent exposure in the onsite and offsite environment. The environmental surveillance criteria are derived from: (1) the collected environmental surveillance data on radionuclides and doses; (2) applicable regulations other than DOE Orders, (3) DOE Order 5400.1 (DOE 1988); and (4) the DOE Environmental Regulatory Guide for Radiological Effluent Monitoring and Environmental Surveillance (DOE 1991). The surveillance program (PNNL 1996a) was established on these criteria and the pathway analyses that provide information on radionuclides and media contributing to human dose. Experience from Hanford Site environmental surveillance activities and studies conducted over the past 45 years has built an invaluable technical background of information for planning and data interpretation.

\subsubsection{Description of Diffuse and Fugitive Emission Sources}

The presently identified actual or potential sources of diffuse and fugitive radionuclide emissions to the environment at the Hanford Site are described in this section. Among the sources that could contribute diffuse and fugitive radionuclide emissions are several kinds of waste handling and disposal facilities used at the Hanford Site such as cribs, ponds, ditches, trenches, retention basins, valve pits, French drains, reverse wells, tanks, and burial grounds. Operating facilities or facilities on standby or that are inactive also could contribute diffuse and fugitive emissions. Table 4-2 shows the numbers of and types of waste sites at Hanford. Each site or facility usually has one or more unique features or characteristics that may contribute to the release of diffuse and fugitive emissions. Features may include passive vents, risers, equipment and personnel access doors, and exhausters. Characteristics may include an undetected leak, unburied waste, or an absence of intrusion barriers. Rates of diffuse and fugitive emissions may be influenced by a variety of environmental conditions, such as: (1) changing atmospheric pressures; (2) wind speed; (3) erosion; (4) evaporation; (5) percolation; (6) biotic intrusion; or (7) wind-caused resuspension.

The general types of sites and facilities and their potential primary sources of diffuse and fugitive emissions are briefly described in sections 4.3.1.1 through 4.3.1.12 that follow.

4.3.1.1 Crib. Low-level liquid wastes were discharged to the ground by structures called cribs. These are generally subsurface systems, similar to sanitary drain fields, that allow the liquid component of the waste to percolate into the soil. The natural properties of the soil are used to remove radioactive material from the effluent water through filtration, ion-exchange, and precipitation reactions. 
Table 4-2

\begin{tabular}{|c|c|}
\hline \multicolumn{2}{|c|}{$\begin{array}{c}\text { Types of Waste and Number of Waste Sites } \\
\text { at the Hanford Site. }\end{array}$} \\
\hline Types of Waste & Number of Waste Sites \\
\hline Nonhazardous & 184 \\
\hline Hazardous & 142 \\
\hline Radioactive & 146 \\
\hline Mixed & 949 \\
\hline Total & 1,421 \\
\hline
\end{tabular}

Many cribs are vented to the atmosphere through engineered structures called vents and pipe risers. These engineered structures promote downward flow of liquids disposed of in the cribs but also provide pathways to the surface and atmosphere. Secondary causes of diffuse and fugitive emissions include erosion, uptake and intrusion by biota, followed by wind-caused resuspension.

4.3.1.2 Pond. Ponds are used to manage the large quantities of water (i.e., cooling water and chemical-sewer wastewater) associated with chemical processing operations. Normally, these liquid effluents are uncontaminated. The ponds allow percolation of the liquid effluent into the soil column. Diffuse and fugitive emissions from ponds are caused primarily by wind-caused resuspension.

4.3.1.3 Ditch. A ditch is an open, unlined excavation used for disposing of liquid effluents or transporting liquid effluents to ponds for disposal. Diffuse and fugitive emissions from ditches are primarily caused by wind-caused resuspension.

4.3.1.4 Trench. Early disposal practices included disposing of liquid effluents into unlined trenches and filling them with soil over time. These were mostly replaced by cribs such as the BC-cribs. Diffuse and fugitive emissions from trenches are primarily caused by erosion, uptake and intrusion by biota, followed by wind-caused resuspension.

4.3.1.5 Retention Basin. Similar to trenches, retention basins generally were lined with concrete and used to hold liquids before routing them to ditches or ponds. Diffuse and fugitive emissions from retention basins are caused primarily by wind-caused resuspension. 
4.3.1.6 Diversion Box. A diversion box is usually an underground, concrete structure formed around a junction of transfer lines carrying liquid effluent. When diversion boxes are accessed for operations or maintenance, radioactively contaminated material may be released in the form of diffuse and fugitive emissions.

4.3.1.7 Valve Pit. A valve pit is similar in structure to a diversion box, but contains piping valves. When valve pits are accessed for maintenance or operations, radioactively contaminated material may be released in the form of diffuse and fugitive emissions.

4.3.1.8 French Drain and Reverse Well. A French drain is a rock-filled encasement inserted in the ground. A reverse well is an ordinary well used for mixing liquid waste with groundwater. These subsurface systerns disposed of potentially contaminated liquid waste by promoting percolation into the soil. The natural filtration properties of the soil removed radioactive material from effluent water. Diffuse and fugitive emissions from French drains and reverse wells may occur through erosion, uptake and intrusion by biota, followed by wind-caused suspension.

4.3.1.9 Tank. A tank generally is a large reinforced metal structure that receives liquid effluent for storage. Examples are the double-shell tanks (DSTs) and single shell tanks (SSTs) in the 200 Areas, which typically are arrayed in clusters called tank farms. Pathways for diffuse and fugitive emissions from tanks include vents and exhausters open to the atmosphere and deposition and resuspension.

4.3.1.10 Burial Ground. Burial grounds are shallow trenches in which contaminated solid waste is buried. This waste is generated by various activities on the Hanford Site. Waste packaging procedures and burial practices used depend on the type of waste. Diffuse and fugitive emissions occur at burial grounds through direct release to the atmosphere before the waste is buried, followed by erosion, uptake and intrusion by biota, and wind-caused resuspension.

4.3.1.11 Decontamination and Decommissioning (D\&D) Activities. Westinghouse Hanford (WHC) and Bechtel Hanford (BHI) activities also involve decontaminating and decommissioning retired facilities, equipment, and waste disposal sites. These activities are aimed at preventing the release or spread of contamination and reducing the number of acres of surface contamination. In some cases, contaminated material may be exposed to the atmosphere, but steps are taken to isolate and reduce spreading of contamination as it is identified. Diffuse and fugitive emissions may be caused by exposing contaminated surfaces such as the inside of contaminated structures. Proper planning and controls minimize these exposures. In addition, monitors around demolition sites confirm that controls are effective. To date, no significant increases at demolition sites have been observed. The majority of these activities have been transferred to BHI.

4.3.1.12 Radioactively Contaminated Surface Areas. All of the following radioactive contaminated areas are routinely surveyed: burial grounds, cribs, trenches, retention basins, and unplanned release sites. The surveys are performed at least annually, but more 
frequently when needed. The number of surface-contaminated acres varies. The number is fixed because there is a continuing effort to clean, stabilize, or remediate known cuntaminated areas while new areas of contamination are continuing to be identified. Newly identified contamination may be because of preexisting contamination that has migrated, by way of wind or biological intrusion, to previously uncontaminated areas or because the radiological criteria have become more stringent. Eighty percent of all areas of surface contamination are estimated to have dose rates less than $1 \mathrm{mrem} / \mathrm{h}$.

Contaminated areas are posted as "Surface Contamination Area," "Radiologically Controlled Area," or "Underground Radioactive Material." Radiologically controlled areas are large areas with a potential for speck contamination. Surface contamination areas have more widespread contamination. "Underground Radioactive Material" signs mark cribs, burial grounds, covered trenches, and ponds, but not underground plumes that extend away from these sites. If an area has surface and underground contamination, such as a surface contaminated crib, it will have both postings. The general location, by area, and the approximate area of surface contamination and underground contamination are shown in Table 4-3. Diffuse and fugitive emissions from areas of surface contamination are caused primarily by erosion, uptake and intrusion by biota, followed by wind-caused resuspension.

\subsubsection{Description of Specific Diffuse and Fugitive Emission Sources}

4.3.2.1 100 Areas' Inactive Reactor Sites. The inactive reactor sites include the: $100-\mathrm{B} / \mathrm{C}$ Area, 100-D Area, 100-F Area, and 100-H Area. These reactor sites are currently undergoing or have plans for decontamination and decommissioning. The potential sources for diffuse and fugitive emissions include personnel and equipment passing through access doors, decontamination activities, and decommissioning activities.

4.3.2.2 100-K Area Basins. Two identical reactors are located in the 100-K Area. One reactor is in the 100-KE Area while the other is in the 100-KW Area. The reactors and their support facilities were constructed between 1952 and 1954, beginning service in 1955. The $100-\mathrm{KW}$ Area reactor ceased operating in 1970 and the $100-\mathrm{KE}$ reactor in 1971.

The 100-KE and $-\mathrm{KW}$ reactor systems underwent decontamination and decommissioning (D\&D) after shutdown. Most of the fuel was sent to 200 East Area for processing. After the initial D\&D was completed, the fuel storage basins within the 105-KE and 105-KW buildings were modified to store $\mathrm{N}$ Reactor irradiated fuel. Storing this fuel began in 1975 at $105-\mathrm{KE}$ and in 1981 at $105-\mathrm{KW}$; fuel is still stored in both basins.

Shipments of fuel to the basins from 100-N Area ceased in 1989. 
Table 4-3

\begin{tabular}{|c|c|c|}
\hline \multicolumn{2}{|c|}{ Hanford Site Surface and Underground Contamination. } \\
\hline Hanford Site Area & $\begin{array}{c}\text { Surface Contamination } \\
\text { ha (acres) }\end{array}$ & $\begin{array}{c}\text { Underground Radioactive }_{\text {Material }^{\mathbf{b}}} \\
\text { ha (acres) }\end{array}$ \\
\hline 100 Areas & $51(129)$ & $178(446)$ \\
\hline 200 Areas $^{\mathrm{c}}$ & $2,459(6,148)$ & $828(2,070)$ \\
\hline 300 Area (north) & $21(52)$ & $13(33)$ \\
\hline 600 Area & 0 & $6(15)$ \\
\hline Total & $2,531(6,328)$ & $1025(2,563)$ \\
\hline
\end{tabular}

Notes:

a Includes areas posted as "Surface/Soil Contamination Areas" or as "Radiological Controlled Areas" and areas that have both underground and surface/soil contamination.

b Only includes areas with underground contamination. Does not include areas that had surface and underground radioactive material.

c Includes tank farms, BC controlled zone, and waste disposal facilities outside the 200 East and 200 West boundaries which received wastes from the 200 East and 200 West facilities (e.g. 216-A-25, 216-B-3-3. 216-S-19, 216-U-11, etc...).

The primary radionuclides that may be included in diffuse and fugitive emissions from the $100-\mathrm{K}$ Basins include ${ }^{90} \mathrm{Sr},{ }^{137} \mathrm{Cs},{ }^{239,240} \mathrm{Pu},{ }^{241} \mathrm{Pu}$, and ${ }^{241} \mathrm{Am}$. The potential release points for diffuse and fugitive emissions include personnel and equipment access doors, which are normally closed.

4.3.2.3 N Reactor. The $\mathrm{N}$ Reactor was designed to produce special nuclear materials (SNM) and byproduct steam for electrical power generation. It began operations in 1963, and ceased operating in January 1987. The 100-N Area reactor complex consists of 126 buildings for reactor operations, maintenance, support facilities, and offices. Some of the more important reactor operations facilities with radioactive materials are identified below.

The 105-N Reactor Building contains the reactor, the reactor control room, the fuel loading area, and the spent fuel storage basin. The $\mathrm{N}$ Reactor is a graphite-moderated, light-water-cooled, horizontal-pressure-tube nuclear reactor designed to produce SNM using slightly enriched uranium. Radioactively contaminated facilities associated with the N Reactor are:

- The 1301-N and 1325-N Liquid Waste Disposal Facilities consists of a crib and trench. 
- The 109-N Heat-Exchanger Building transferred the heat from the primary loop to the secondary loop through steam generators.

- The 117-N Filter Building houses charcoal and HEPA filters.

- The 107-N Basin Recirculating Building removes radionuclides from the N Basin Fuel Storage Facility water by filtering and demineralizing it.

The primary radionuclides that may be included in diffuse and fugitive emissions from t. $100-\mathrm{N}$ facilities include ${ }^{60} \mathrm{Co},{ }^{90} \mathrm{Sr},{ }^{137} \mathrm{Cs}$, and ${ }^{239,240} \mathrm{Pu}$. The potential primary diffuse and fugitive emission release points include railroad roll-up doors, personnel and other equipment access doors (all of which are normally closed), and inactive exhaust vents and risers.

A special case at the Hanford Site is the N Springs, which discharge low-level contamination (primarily ${ }^{90} \mathrm{Sr}$ ) into the Columbia River. These springs are perhaps more closely monitored than any comparable component of the Hanford Site ecosystem. Other means of diffuse and fugitive emissions include erosion, uptake or intrusion of biota, and wind-caused resuspension.

4.3.2.4 Plutonium-Uranium Extraction (PUREX) Facility. The PUREX Plant is a collection of buildings and facilities located in the 200 East Area at the Hanford Site. The main building, 202-A, is a heavily shielded, reinforced concrete structure known as a canyon. This building contains the main equipment that was in the PUREX process of chemically separating and purifying actinides from the irradiated nuclear fuel.

Radionuclides primarily associated with the PUREX Plant include ${ }^{14} \mathrm{C},{ }^{85} \mathrm{Kr},{ }^{90} \mathrm{Sr}$, ${ }^{106} \mathrm{Ru},{ }^{129} \mathrm{I},{ }^{137} \mathrm{Cs},{ }^{147} \mathrm{Pm},{ }^{238} \mathrm{Pu},{ }^{239,240} \mathrm{Pu},{ }^{241} \mathrm{Pu}$, and ${ }^{241} \mathrm{Am}$. Potential diffuse and fugitive emission release points include access doors and the inlet-exhaust ventilation system, all of which are controlled.

4.3.2.5 Uranium-TriOxide Plant. The Uranium-TriOxide $\left(\mathrm{UO}_{3}\right)$ Plant, located in the 200 West Area, produced $\mathrm{UO}_{3}$ powder by calcining uranyl nitrate solutions from the PUREX Plant. The $\mathrm{UO}_{3}$ powder was sealed in steel drums for shipment offsite.

Uranium was formerly the potential primary source of radioactive diffuse and fugitive emissions from the $\mathrm{UO}_{3}$ Plant. Since the $\mathrm{UO}_{3}$ Plant's deactivation, only trace amounts of $\mathrm{UO}_{3}$ remain, all of which is sealed inside of equipment. $\mathrm{UO}_{3}$ 's deactivation has significantly reduced the potential diffuse and fugitive emissions from the facility. The noble gases radon and thoron remain as the only source of diffuse and fugitive emissions, due to residual contamination sealed inside $\mathrm{UO}_{3}$ 's equipment. Potential diffuse and fugitive emission release points include access doors all of which are restricted and controlled.

4.3.2.6 Plutonium Finishing Plant (PFP). This facility is located in the 200 West Area and is used to recover, stabilize, and store plutonium. Recovered plutonium nitrate and plutonium nitrate solutions received from the PUREX Plant were reduced to plutonium 
dioxide. The reduction process stabilized the plutonium into the state best suited for longterm storage. PFPs mission no longer includes the production of finished plutonium metal.

Radionuclides primarily associated with PFP include ${ }^{239,240} \mathrm{Pu},{ }^{241} \mathrm{Pu}$, and ${ }^{241} \mathrm{Am}$. Potential diffuse and fugitive emission release points from PFP include access doors and the inlet-exhaust ventilation system, all of which are controlled.

4.3.2.6.1 234-5Z Building (active). The 234-5Z Building is also known as PFP or the 234-5 Building. The 234-5Z Building basement mostly consists of pipe tunnels carrying drain piping. The first floor houses the following: (1) two plutonium processing lines (Remote Mechanical A and Remote Mechanical C Lines) and their control rooms, (2) scrap stabilization gloveboxes, (3) plutonium storage vaults, and (4) the plutonium nitrate feed load-in/load-out (LI/LO), blending, and storage facilities.

Radionuclides primarily associated with the $234-5 \mathrm{Z}$ Building include ${ }^{239,240} \mathrm{Pu}$ and ${ }^{241} \mathrm{Am}$. Potential diffuse and fugitive release points include access doors and the inlet-exhaust ventilation system, all of which are controlled.

4.3.2.6.2 236-Z Building. The $236-Z$ Building, commonly known as PRF (Plutonium Reclamation Facility), is located south of the southeastern corner of the 234-5Z Building, connected to it by the $242-Z$ Building. The building air exhausts through the $291-Z-1$ stack.

Radionuclides primarily associated with the $236-\mathrm{Z}$ Building include ${ }^{239,240} \mathrm{Pu}$ and ${ }^{241} \mathrm{Am}$. Potential diffuse and fugitive release points include access doors and the inlet-exhaust ventilation system, all of which are controlled.

4.3.2.6.3 232-Z Building. The 232-Z Building houses the layaway Contaminated Waste Recovery Process. It was commonly called the "Incinerator." The Contaminated Waste Recovery Process was partially decontaminated and decommissioned in 1984.

Radionuclides primarily associated with the $232-\mathrm{Z}$ Building include ${ }^{239,240} \mathrm{Pu}$ and ${ }^{241} \mathrm{Am}$. Potential diffuse and fugitive ernission release points include access doors and inlet-exhaust ventilation systems, all of which are controlled.

4.3.2.6.4 241- $Z$ and $242-Z$ Buildings. The $241-Z$ and $242-Z$ Buildings are designated as waste treatment facilities. $241-Z$ houses sumps, which are five separate enclosures or ventilated cells, each containing a 5,300-gal $(20,000-\mathrm{L})$ tank used to accumulate the liquid wastes generated by PFP before being transferred to the tank farms. The $241-Z$ is ventilated by the $296-Z-3$ stack.

The 242-Z Building houses abandoned waste treatment process equipment, once used to recover americium. This facility was permanently shut down after a process upset in 1979 spread contamination and caused irreparable equipment damage inside. The structural 
integrity of the facility was not compromised, however. The facility was then decontaminated extensively before being placed in layaway pending decommissioning work. The 242-Z Building shares the main ventilation system of the $234-5 Z$ and $236-Z$ Buildings, exhausting its building air through the 291-Z-1 stack.

Radionuclides primarily associated with the $241-\mathrm{Z}$ and $242-\mathrm{Z}$ Buildings include ${ }^{239,240} \mathrm{Pu}$ and ${ }^{241} \mathrm{Am}$. Potential diffuse and fugitive emission release points include access doors and the inlet-exhaust ventilation system, all of which are controlled.

4.3.2.6.5 2736-ZB Building. The 2736-ZB shipping area and receiving area both accommodate a maximum of 100 shipping containers, each of which is about the size of a 55-gal (210-L) drum. Adequate spacing is provided between containers to meet WHC criticality prevention requirements, personnel exposure specifications, and corridor access standards to emergency staging areas. The two areas are physically separated by a wall.

Radionuclides primarily associated with the $2736-\mathrm{ZB}$ Building include ${ }^{239,240} \mathrm{Pu}$ and ${ }^{241} \mathrm{Am}$. Potential diffuse and fugitive emission release points include access doors and the inlet-exhaust ventilation system, all of which are controlled.

4.3.2.6.6 216-Z-20 Crib Effluent Facility. The 216-Z-20 Crib was constructed and commissioned for use in September 1981 to dispose of waste water previously discharged to the 216-U-10 Pond through the 216-Z-19 Ditch. Use of the crib allowed the radioactively contaminated 216-Z-19 Ditch and 216-U-10 Pond to be decommissioned.

The $216-Z-20$ Crib was designed and constructed to dispose of potentially radioactive liquid effluents from the $231-Z, 232-Z, 236-Z, 242-Z, 291-Z, 234-5 Z$, and $2736-Z B$ Buildings. The same building effluent headers and piping that served the 216-Z-19 Ditch were used. These headers and piping contain some residual contamination. The 207-Z Seepage Basin, later renamed 216-Z-21 Seepage Basin, was designed and constructed to dispose of uncontaminated water from the storm sewer north of the 234-5Z Building, water-tank overflow, and swamp-cooler waste water.

The $216-Z-20$ Crib is approximately $460 \mathrm{~m}(1,500 \mathrm{ft})$ long with a cross section at the bottom that is approximately $3 \mathrm{~m}(10 \mathrm{ft})$ wide. Gravel was used as backfill to distribute effluents throughout the crib. A vapor barrier was placed above the gravel backfill. Soil was placed over the top to bring the area back to the surrounding grade.

Radionuclides primarily associated with the $216-\mathrm{Z}-20$ Crib include ${ }^{239.240} \mathrm{Pu}$ and ${ }^{241} \mathrm{Am}$. Diffuse and fugitive emissions may be released by way of vents, risers, erosion, biotic uptake and intrusion, and wind-caused resuspension.

4.3.2.7 T Plant. Originally, T Plant was a fuel separations facility using the bismuth phosphate process. Now the facility is used for radioactive decontamination and repair of equipment. This facility currently is active. 
T Plant is located in the 200 West Area of the Hanford Site. Buildings, structures, or special facilities included as part of this facility are the 221-T and 2706-T Buildings, deactivated 221-T Building Head-End, and 214-T Chemical Storage Building. Ancillary buildings, structures, and areas included are the 271-T, 291-T and 221-TA Buildings, and the 211-T Chemical Storage Area. Decontamination processes are conducted in the 221-T and 2706-T Buildings. The 221-T Building Head-End was used for special experiments and operations. The 214-T Building stores chemicals. The 211-T Chemical Storage Area consists of a permitted pad that stores nonradioactive hazardous waste. The 271-T Building provides office space to WHC staff supporting T Plant operations. The 291-T Building houses the exhaust ventilation fans for the 291-T-1 main stack. The 221-TA Building houses the supply ventilation fans for the 221-T Building canyon.

Radioactive decontamination activities are performed in the canyon area. The canyon area consists of 37 cells and one railroad tunnel door. The railroad tunnel (used for transporting equipment into and out of the canyon, as well as for some decontamination) enters the plant at cell $2 \mathrm{~L}$. A motor-driven rolling steel door, provides railroad canyon access.

Primary radionuclides associated with $\mathrm{T}$ Plant include ${ }^{90} \mathrm{Sr},{ }^{137} \mathrm{Cs}$, and ${ }^{239,240} \mathrm{Pu}$. Potential diffuse and fugitive emission release points include access doors and the inlet-exhaust ventilation system, all of which are controlled.

4.3.2.7.1 2706-T Building. The 2706-T Building is a ground-level structure constructed of prefabricated steel. It has two openings on the west end that are fitted with roll-up metal doors that allow access to the pit areas. Low-level radioactive decontamination activities are performed in these two pits.

Radionuclides primarily associated with the $2706-\mathrm{T}$ Building include ${ }^{90} \mathrm{Sr},{ }^{137} \mathrm{Cs}$, ${ }^{239.240} \mathrm{Pu}$, and ${ }^{241} \mathrm{Am}$. Potential diffuse and fugitive emission release points include access doors, the inlet-exhaust ventilation system, and an outdoor storage area, all of which are controlled.

4.3.2.7.2 221-T Building - Head-End Operations. The 221-T Building head-end consists of a canyon area extending from the basement floor to the roof. This canyon area has several deck levels and a parapet wall. Four floor levels adjacent to the canyon house include: (1) an electrical switchgear room, (2) a chemistry laboratory, (3) office areas, (4) a change room, (5) a lunch room, (6) a control room, (7) an instrument shop, (8) a maintenance shop, (9) and storage areas. In 1994, flow from eight process sewer lines originating in the Head-End was discharged to the 216-T-1 Ditch, which is no longer in use.

Radionuclides primarily associated with $221-\mathrm{T}$ Head-End Operations include ${ }^{90} \mathrm{Sr}$, ${ }^{137} \mathrm{Cs}$, and ${ }^{239.240} \mathrm{Pu}$. Potential diffuse and fugitive emission release points include access doors and inlet-exhaust ventilation system, all of which are controlled. 


\subsection{1-T Building - Pressurized-Water (Cooled) Reactor Fuel Assembly}

Storage. Canyon cell $2 \mathrm{R}$ has been modified to store approximately 76 pressurized-water reactor Core 2 blanket fuel assemblies that were used to power the Shippingport Reactor. Provisions have been made to store the assemblies at the Hanford Site for up to 20 years.

The 221-T Building galleries are maintained at atmospheric pressure, while the 221-T Building canyon area is maintained at a negative pressure with respect to atmosphere. A primary design feature of these systems is to ensure that potentially contaminated canyon air is completely separate from the clean air in the 221-T Building galleries and the 271-T Building, which is the office space connected to the 221-T Building. In addition, the canyon air system is operated at negative pressures with respect to the other systems.

The 271-T Building is adjacent to the 221-T Operating Gallery. While most of this building is used for office space, portions are used by T-Plant Operations.

Radionuclides primarily associated with 221-T-PWR Fuel Assembly Storage include ${ }^{90} \mathrm{Sr},{ }^{137} \mathrm{Cs}$, and ${ }^{239.240} \mathrm{Pu}$. Potential diffuse and fugitive emission release points include access doors and the inlet-exhaust ventilation system, all of which are controlled.

4.3.2.8 Transuranic Waste Storage and Assay Facility. Originally, the function of the 224-T Building was to purify plutonium nitrate by the lanthanum-fluoride process. The plant remained inactive following phase-out of the bismuth phosphate plants until the early 1970s. At that time, the building was modified for storage of plutonium scrap in liquid and solid forms.

In 1984, the 224-T Building was chosen to house the transuranic (TRU) waste storage and assay operation. The Transuranic Waste Storage and Assay Facility (TRUSAF) operation consists of a nondestructive analysis of TRU waste. The analysis is used as an overview for sealed, certified, contact-handled, TRU solid-waste packages, to verify general compliance with the Waste Isolation Pilot Plant (WIPP) Waste Acceptance Criteria. Those containers meeting WIPP acceptance criteria are stored at 224-T and maintained in a manner to retain their certification pending shipment to WIPP. The TRUSAF operation also performs a sorting function for the PFP.

In 1985, the removal of plutonium scrap from 224-T was completed, and the building was officially designated as the TRUSAF.

Radionuclides primarily associated with TRUSAF include ${ }^{90} \mathrm{Sr}$, ${ }^{137} \mathrm{Cs}$, and ${ }^{239.240} \mathrm{Pu}$. Potential diffuse and fugitive emission release points include access doors and the inlet-exhaust ventilation system, all of which are controlled.

4.3.2.9 B Plant. B Plant was originally designed to chemically process spent nuclear fuels. Radiological containment and confinement features were incorporated in the various facilities and support systems to prevent exposure of plant personnel and the general public to excessive radiation. The plant was then modified to separate strontium and cesium from the 
fission product waste stream following plutonium and uranium recovery from irradiated reactor fuels in the PUREX Plant. The recovered purified and concentrated strontium and cesium solutions were then transferred to the Waste Encapsulation Storage Facility (WESF) for conversion to solid compounds, encapsulation, and interim storage. After strontium and cesium removal, the remaining waste was transferred from B Plant to the tank farms.

B Plant is an operating facility used to ensure safe storage and management of the WESF cesium and strontium capsules and a radiological inventory remaining in the plant from previous campaigns. There are currently no production activities at B Plant, but several systems are operating systems to perform the current B Plant mission. B Plant receives and stores various chemicals from: (1) generation of demineralized water; (2) and conditioning of water used in HVAC units.

B Plant consists of three main buildings. They are the 221-B Processing Building, 271-B Service and Office Building, and 225-B Building (WESF). The 221-B Process Building and its attached 271-B Service Building were constructed in 1943. Construction of the WESF was completed in 1974.

Radionuclides primarily associated with B Plant include ${ }^{90} \mathrm{Sr}$ and ${ }^{137} \mathrm{Cs}$. Potential diffuse and fugitive emission release points include access doors and the inlet-exhaust ventilation system, all of which are controlled.

4.3.2.10 222-S Facility. The 222-S Laboratory Complex is located in the southeast corner of the 200 West Area. The facility is composed of the main laboratory complex (222-S) and a number of ancillary buildings and structures.

4.3.2.10.1 222-S Laboratory. The 222-S Laboratory is a two-story, aboveground building with a subterranean service level. This building is divided into laboratory support spaces, office spaces, a multi-curie wing, seven environmental hot cells, and supplemental service areas. The building is designed with its own waste disposal facility, decontamination facility, fire protection and alarm system, ventilation system, and radiation monitoring systems.

The 222-S Laboratory Annex houses the maintenance shop, instrument shop, and the counting room filter building.

Radionuclides primarily associated with the $222-\mathrm{S}$ Laboratory include ${ }^{90} \mathrm{Sr}$ and ${ }^{137} \mathrm{Cs}$. Potential diffuse and fugitive emission release points include access doors and the inlet-exhaust ventilation system, all of which are controlled.

4.3.2.10.2 222-SA Laboratory. The 222-SA Laboratory is a five-wide modular building located southeast of the 222-S Laboratory. Part of this laboratory prepares nonradioactive standards for Hanford Site laboratories. The other section of the laboratory is used for cold-process development work and standards preparation. The 222-SA Laboratory only has the potential for nonradioactive diffuse and fugitive emissions. 
4.3.2.10.3 2716-S Storage Building. The 2716-S Storage Building, located south of the 222-S Laboratory, is partitioned off for the storage of acids and bases. It provides both long- and short-term storage capability for laboratory materials and contains no radioactive materials.

There are no radioactive diffuse fugitive emissions from the 2716-S Storage Building.

4.3.2.10.4 207-SL Retention Basin. The 207-SL Retention Basin acts as a temporary holding facility for potentially radioactive or hazardous liquid effluents before being transferred by means of a cross-site pipeline to the Treated Effluent Disposal Facility, located in the 200 East Area. Waste water from the laboratory, normally free of radioactive and hazardous chemical contamination, is routed to the 207-SL Retention Basin. This facility is a covered, below-grade concrete structure, directly east of the 222-S Building. Two 25,000-gal (95,000-L) compartments allow batch collection, sampling, and discharge of the waste. Three 20,000-gal (75,708-L) storage tanks were added in 1994 to improve waste transfer and storage capabilities.

Radionuclides primarily associated with the $207-$ SL Retention Basin include ${ }^{90} \mathrm{Sr}$, ${ }^{137} \mathrm{Cs}$, and ${ }^{239 / 240} \mathrm{Pu}$. Potential diffuse fugitive release points include access doors and seams in the basin cover blocks.

4.3.2.10.5 219-S Waste Handling Facility. The 219-S Waste Handling Facility collects liquid waste generated by the 222-S Laboratory operations that is contaminated radioactively and/or with hazardous chemicals. This facility consists of two below-grade vaults ( $A$ and $B$, also called cells) containing; three stainless-steel tanks, a transite building, the pipe trench and operating gallery, and an attached concrete-walled sample gallery. Tanks TK-101 and TK-102 are in vault $A$ and tank TK-103 is in vault $B$.

Radionuclides primarily associated with the 219-S Waste Handling Facility include ${ }^{90} \mathrm{Sr}$ and ${ }^{137} \mathrm{Cs}$. Potential diffuse fugitive release points include access doors and seams in the vault cover blocks.

4.3.2.10.6 222-SB Filter Building. The 222-SB Filter Building, located south of the 222-S Building, houses 96 HEPA filters which provide final filtration for the 222-S Laboratory. Under normal operation of the ventilation system, three electrically powered fans exhaust air from the 222-S Laboratory. Exhaust air leaves the 222-S Building through the 296-S-21 stack. Emergency exhaust ventilation is provided by the 222-SE Filter Building, in the event that two or more exhaust fans fail to operate.

Radionuclides primarily associated with the 222-SB Filter Building include ${ }^{90} \mathrm{Sr}$ and ${ }^{137} \mathrm{Cs}$. Potential diffuse fugitive emission release points include access doors and the inlet-exhaust ventilation system, all of which are controlled. 
4.3.2.10.7 222-SC Filter Building. The 222-SC Filter Building, located north of the 222-S Laboratory, contains the second and third stage HEPA filtration for hot cells 1-A, 1-E-1, 1-E-2, 1-F, and 11-A-1 through 11-A-6. The hot cells in rooms 1-A, 1-E, 1-E, 1-F, and 11-A are serviced by the main building supply and exhaust ventilation. The 222-SC Filter Building houses five parallel pairs of HEPA filters, which provide filtration to hot cell exhaust air before it enters the main exhaust plenum and final filtering in the 222-SB Filter Building. A total of four stages of HEPA filtration are provided for the hot cell ventilation exhaust.

Radionuclides primarily associated with the 222-SC Filter Building include ${ }^{90} \mathrm{Sr}$ and ${ }^{137} \mathrm{Cs}$. Potential diffuse and fugitive emission release points include access doors and the inlet-exhaust ventilation system, all of which are controlled.

4.3.2.10.8 222-SE Filter Building. The 222-SE Filter Building, located south of the 222-S Building, is a building that houses 56 HEPA filters. This building provides redundant backup filtering capabilities for the 222-S Laboratory exhaust.

Radionuclides primarily associated with the 222-SE Filter Building include ${ }^{90} \mathrm{Sr}$ and ${ }^{137} \mathrm{Cs}$. Potential diffuse and fugitive emission release points include access doors and the inlet-exhaust ventilation system, all of which are controlled.

4.3.2.10.9 218-W-7 Dry Waste Burial Vault. The 218-W-7 Dry Waste Burial Vault is located $40 \mathrm{ft}$ southeast of $222-\mathrm{S}$. This underground concrete vault was removed from service around 1960. It was used primarily for disposal of plutonium-contaminated dry-hood waste generated by the 222-S Laboratory. Access to the tank is through a locked hatchway.

Plutonium-239 and -240 are the radionuclides primarily associated with the 218-W-7 Dry Waste Burial Vault. A locked access hatchway is the only potential release point for diffuse and fugitive emissions.

4.3.2.10.10 216-S-26 Crib. The 216-S-26 Crib is no longer in service and the pipeline to the crib has been sealed off. All waste water collected in the 207-SL Retention Basins is now discharged to the Treated Effluent Disposal Facility, located in the 200 East Area. Additionally, the 222-SA waste water has been rerouted from the crib to the 207-SL Retention Basins. The line from the 291-S Stack Fan House, which formerly discharged steam condensate to the crib, has been isolated.

Radionuclides historically discharged to the $216-\mathrm{S}-26 \mathrm{Crib}$ include ${ }^{90} \mathrm{Sr},{ }^{137} \mathrm{Cs}$, and ${ }^{239,240} \mathrm{Pu}$. Diffuse and fugitive emissions may be released by way of vents, risers, erosion, biotic uptake and intrusion, and wind-caused resuspension.

4.3.2.11 Waste Verification and Sampling Facility. The Waste Verification and Sampling Facility, the 213-W Building, is located in the 200 West Area of the Hanford Site. The 213-W Building is adjacent to the 272-WA Building (the Operations Support Building) at the 
218-W-5 Burial Grounds at the west end of the 200 West Area. Systems ancillary to the 213-W Building also are included.

The primary function or process associated with the Facility is the verification of waste drums received from waste generators. Because of limited use, it is was transferred to the 200 West Tank Farms Facility in 1995.

Radionuclides primarily associated with the 213 -W Building include ${ }^{90} \mathrm{Sr}$ and ${ }^{137} \mathrm{Cs}$; low-level waste is present also. Potential diffuse and fugitive emission release points include access doors and the inlet-exhaust ventilation system, all of which are controlled.

4.3.2.12 Central Waste Complex. The Central Waste Complex (CWC) is a group of structures located on the west side of 200 West Area exclusion zone. The primary function or process associated with the CWC Facility is the receipt and storage of radioactive and mixed waste (MW), which is currently ongoing. This facility has the potential to generate radioactive and/or hazardous chemical emissions and radioactive and/or hazardous chemical liquid effluent.

Radionuclides associated with the Central Waste Complex are from a wide group of mixed fission, mixed waste, and transuranic radionuclides. Potential diffuse and fugitive emission release points include access doors and the inlet-exhaust ventilation system, all of which are controlled. The facility consists of the following.

4.3.2.12.1 Pu and PCB Storage Facility. This facility is a steel building with concrete curbing within its perimeter. The building has a water-based fire suppression system and continuous air monitors (CAM) for detection of airborne radioactive particulates.

4.3.2.12.2 Low-Flashpoint MW Storage Modules. Each of the low-flashpoint MW storage modules has a catch sump with a holding capacity of 750 gal $(2,800 \mathrm{~L})$. Modules used for storage of TRU-mixed waste also have draft ventilation.

4.3.2.12.3 Mixed-Waste Storage Facilities. The Buildings 2402-WB through 2402-WL, 2402-W, and 2403-W are metal structures with concrete floors, ventilation systems, and water-based fire protection. The concrete floors have provisions to collect any spilled liquids. The facilities have CAMs for detection of airborne radioactive particulates.

4.3.2.12.4 Mixed-Waste Storage Pad. The MW Storage Pad is a $9,000 \mathrm{ft}^{2}\left(320 \mathrm{~m}^{3)}\right.$ concrete storage pad with 6 -in. $(15-\mathrm{cm})$ curbing around its perimeter. The pad has a stormwater collection system that allows for disposing of the stormwater after sampling and analysis.

4.3.2.12.5 Waste Receiving and Storage Pad. The Waste Receiving and Storage Pad is a 61-m-by-46-m (200-ft-by-150-ft) asphalt pad. 
4.3.2.13 Tank Farms. Liquid waste from chemical processing operations containing high concentrations of radionuclides is stored on an interim basis in underground tanks. The Hanford Site tank farms contain 177 tanks (149 SSTs and 28 DSTs) with capacities ranging from 50,000 to 1.2 million gal $(190,000$ to 4.5 million L). Since 1967 , newly generated liquid waste has been stored in DSTs. The SSTs are no longer receiving waste.

The location of all the tank farms is the 200 East and 200 West Areas. Both DSTs and SSTs are present in these areas. Tank farms in the 200 East Area include the A, AX, B, $\mathrm{BX}, \mathrm{BY}, \mathrm{C}$ (SSTs), AN, AZ, AY, AP, and AW (DSTs). Those in the 200 West Area are the S, SX, T, TX, TY, U (SSTs) and the SY (DST).

Hanford Site tank farms comprise transfer routes, diversion boxes, storage vaults, double-contained receiver tanks (DCRT), and evaporators.

A system of underground pipes is used to transfer wastes from the 200 East Area waste generators to the DSTs, between the DSTs, and from the DSTs to treatment and storage units in the 200 East and 200 West Areas.

Radionuclides primarily associated with the tank farms include ${ }^{3} \mathrm{H},{ }^{14} \mathrm{C},{ }^{90} \mathrm{Sr},{ }^{106} \mathrm{Ru}$, ${ }^{125} \mathrm{Sb},{ }^{137} \mathrm{Cs},{ }^{239,240} \mathrm{Pu}$, and ${ }^{241} \mathrm{Am}$. Diffuse and fugitive emission release locations may include vents, risers, access hole covers, inlet-exhaust ventilation systems, diversion boxes, transfer lines, and storage vaults.

4.3.2.13.1 Double-Shell Waste Tanks. The DSTs are of two distinctly different types. The capacity of the first type is 1 to 1.2 million gal $(3.79$ to 4.54 million $\mathrm{L}$ ), and is designed for long-term storage (up to 50 years) of high-activity mixed waste.

Twenty-four 1.2-million-gal (4.5-million-L) non-aging waste DSTs and four 1.0-million-gal (3.8-million-L) aging waste DSTs have been built. For efficiency during construction and operation, the 1 -million-gal tanks were grouped into six tank farms.

The second type of DST is smaller, with storage capacities ranging from 800 to $45,000 \mathrm{gal}(3,028$ to $170,370 \mathrm{~L})$. These tanks were used primarily for lag storage of waste before transfer to the larger tanks or to other facilities. These smaller tanks are called DCRTs.

All DSTs were fabricated as three concentric tanks. Waste is stored in the freestanding primary tank. The secondary tank sits on a concrete pad. The completely enclosed annulus serves as a containment barrier if the primary tank should leak. The annulus is ventilated and continually monitored for evidence of primary tank leakage. The third tank is a concrete shell that encloses the sides of both primary and secondary tanks for additional containment, radiation shielding, and structural support.

Ancillary equipment also is present, such as transfer lines between tank farms and DCRTs, valve pits, diversion boxes, and tank-farm piping. 
4.3.2.14 242-A Evaporator. The 242-A Evaporator complex is located in the 200 East Area of the Hanford Site. The 242-A Building is located south of the 241-A and 241-AX Tank Farms and north of the 242-AW Tank Farm. The 242-A Building contains the evaporator vessel and supporting process equipment. The building ventilation exhaust fans and HEPA filter housings are located on the south side of the building. An emergency diesel generator is located on the south side of the building. Raw water, steam, and electrical power are provided to the 242-A Building from existing service facilities in the 200 East Area.

In general, the 242-A Evaporator Facility can be divided into three areas: process, service, and operating. The process area includes: the evaporator room, pump room, condenser room, and ion exchange enclosure. The service area includes: the aqueous make-up (AMU) room, loadout and hot equipment storage room, loading room, and HVAC room. The operating areas include: the control room, personnel change rooms, lunchroom, office, and storage rooms.

Radionuclides primarily associated with $242-\mathrm{A}$ include ${ }^{3} \mathrm{H},{ }^{14} \mathrm{C},{ }^{90} \mathrm{Sr},{ }^{106} \mathrm{Ru},{ }^{125} \mathrm{Sb}$, ${ }^{137} \mathrm{Cs},{ }^{239.240} \mathrm{Pu}$, and ${ }^{241} \mathrm{Am}$. Potential diffuse and fugitive emission release points include access doors and the inlet-exhaust ventilation system, all of which are controlled.

4.3.2.15 242-S Evaporator. The 242-S Evaporator, currently on standby, is located in the 200 West Area, west of the 241-SY Tank Farm. It consists of an evaporator vessel and supporting process equipment. The building ventilation exhaust fans and HEPA filter housings are on the north side of the building. An emergency diesel generator is on the south side of the building. Raw water, steam, and electrical power are provided from existing service facilities in the 200 East Area. There are three main areas: process, service, and operations. The process area includes: the evaporator room, pump room, condenser room, and ion-exchange enclosure. The service area includes: the Aqueous Make-up (AMU) room, loadout and hot equipment storage room, loading room, and the HV.AC room. The operating areas include: the control room, personnel change rooms, lunchroom, offices, and storage rooms.

Radionuclides primarily associated with $242-\mathrm{S}$ include ${ }^{3} \mathrm{H},{ }^{14} \mathrm{C},{ }^{90} \mathrm{Sr},{ }^{106} \mathrm{Ru},{ }^{125} \mathrm{Sb},{ }^{129} \mathrm{I}$, ${ }^{137} \mathrm{Cs},{ }^{239,240} \mathrm{Pu}$, and ${ }^{241} \mathrm{Am}$. Potential diffuse and fugitive emission release points include access doors and the inlet-exhaust ventilation system, all of which are controlled.

4.3.2.16 242-T Evaporator. The 242-T Evaporator, currently inactive, is located in the 200 West Area, east of the 241-TX and 241-TY Tank Farms. It consists of evaporator vessel and supporting process equipment. The building ventilation exhaust fans and HEPA-filter housings are on the north side of the building. An emergency diesel generator is on the south side of the building. Raw water, steam, and electrical power are provided from existing service facilities in the 200 East Area. There are three main areas: process, service, and operations. The process area includes: the evaporator room, pump room, condenser room, and ion-exchange enclosure. The service area includes: the AMU room, loadout and hot equipment storage room, loading room, and the HVAC room. The operating 
areas include: the control room, personnel change rooms, lunchroom, offices, and storage rooms.

4.3.2.17 Grout Treatment Facility (GTF). The GTF combined low-level radioactive liquid waste with a cement mixture that is pumped into disposal vaults. The GTF was placed in cold standby in 1993.

Radioactive constituents primarily associated with GTF include ${ }^{90} \mathrm{Sr}$, ${ }^{137} \mathrm{Cs}$, and other low-level radioactive waste from the tank farms. Potential diffuse and fugitive emission release modes include vents, risers, and the inlet-exhaust ventilation system.

4.3.2.18 Low-Level Burial Grounds. The active low-level radioactive solid waste burial grounds are located in the 200 Areas of the Hanford Site. The active sites to be considered are those addressed in the Part A, Dangerous Waste Permit Application for the Low-Level Burial Grounds (DOE/RL 1988). In the 200 West Area, the burial grounds to be considered include 218-W-5, 218-W-6, 218-W-3A, 218-W-3AE, 218-W-4B, and 218-W-4C. In the 200 East Area, the burial grounds to be considered include 218-E-10 and 218-E-12B.

The entire array of low-level radionuclides from operations on the Hanford Site is associated with the Low-Level Burial Grounds. Diffuse and fugitive emissions may be released by way of open burials (before covering), ruptured containment vessels, biotic intrusion, erosion, and wind-caused resuspension.

4.3.2.18.1 Burial Ground 218-W-3A. Burial Ground 218-W-3A began receiving waste in 1970. It consists of 61 trenches covering 50.3 acres (20.4 hectares). Waste stored or disposed of in this burial ground includes mixed, transuranic, low-level, and retrievable waste. Other types of waste include ion-exchange resins and industrial waste (such as failed equipment, tanks, pumps, ovens, agitators, heaters, hoods, jumpers, vehicles, and accessories). This burial ground used to store spent fuel.

4.3.2.18.2 Burial Ground 218-W-3AE. Burial Ground 218-W-3AE began receiving waste in 1981 . It consists of 31 trenches covering 49.4 acres (20 hectares). Waste in this burial ground includes low-level and mixed waste. Other types of waste placed in this burial ground include rags, paper, rubber gloves, disposable supplies, broken tools, and industrial waste.

4.3.2.18.3 Burial Ground 218-W-4B. Burial Ground 218-W-4B began receiving waste in 1968. It consists of 13 trenches and 12 caissons covering 8.6 acres ( 3.5 hectares). The trenches contain mixed and retrievable TRU waste and were filled before 1980 . Caisson Alpha 4 is believed to contain mixed waste.

4.3.2.18.4 Burial Ground 218-W-4C. Burial Ground 218-W-4C began receiving waste in 1978. It consists of 69 trenches covering 51.7 acres (20.9 hectares). Some of the waste in this facility is TRU, mixed, and low-level. Other types of waste placed in these 
trenches include contaminated soil, decommissioned pumps, pressure vessels and hardware, and stored spent fuel. Some of the trenches are designed for retrievable storage of waste.

4.3.2.18.5 Burial Ground 218-W-5. Burial Ground 218-W-5 began receiving waste in 1986. It consists of 35 trenches (with room to expand to 57 trenches) covering 84.0 acres ( 34 hectares). The trenches contain low-level mixed waste that includes lead bricks and shielding. Low-level waste also was placed in these trenches.

4.3.2.18.6 Burial Ground 218-W-6. Burial Ground 218-W-6 has not received any waste to date. When developed, this burial ground will consist of 35 trenches and cover approximately 44.5 acres (18 hectares).

4.3.2.18.7 Burial Ground 218-E-10. Burial Ground 218-E-10 began receiving waste in 1960 . It consists of 18 trenches covering 56.7 acres (22.9 hectares). Waste was received from PUREX, B Plant, and $\mathrm{N}$ Reactor. The waste includes low-level and low-level mixed waste such as dragoff waste, failed equipment, and industrial waste.

4.3.2.18.8 Burial Ground 218-E-12B. Burial Ground 218-E-12B began receiving wastes in 1967. It consists of 94 trenches covering about 173 acres (70 hectares). Areas are set aside for future expansion of this burial ground. Some of the waste contained in this burial ground is TRU, mixed, and low-level. Trench 94 contains decommissioned U.S. Navy submarine reactor compartments.

4.3.2.19 300 Area Waste Service Facility. The 340 Complex is located in the 300 Area of the Hanford Site near the Columbia River. The 340 Complex was constructed to collect, store, and transport radioactive liquid wastes. Buildings 324, 325, 326, 327, and 329 were built in the mid-1950's and can discharge liquid radioactive waste to the 340 Complex via the Radioactive Liquid Waste System. Potentially radioactive wastes can be discharged to the 340 Complex via the Retention Process Sewer (RPS). Historically, the 308 building discharged wastes to the RPS, but is no longer able to. All facilities generating waste destined for the 340 Complex are managed by PNNL, except for the 308 building, which is managed by WHC. Only the 324 and 325 buildings can accumulate wastes in tanks and then pump the wastes to the complex.

Uranium, ${ }^{60} \mathrm{Co},{ }^{90} \mathrm{Sr},{ }^{137} \mathrm{Cs},{ }^{238} \mathrm{Pu},{ }^{239 / 240} \mathrm{Pu}$, and ${ }^{241} \mathrm{Am}$ are the most significant radionuclides potentially present at the 340 Complex. Potential diffuse and fugitive emission release points include access doors and the inlet-exhaust ventilation system, all of which are controlled.

4.3.2.20 300 Area N Reactor Fuels Fabrication Facility. The N Reactor Fuels Fabrication Facility is located in the 300 Area of the Hanford Site. It consists of 16 buildings, two tank farms, and associated pipe trenches and drains. The structures are located on the north side of the 300 Area. The 300 Area Fuels Fabrication facilities began operation in 1944 with some structures being added in the 1950's and 1960's. The following sections provide information on each of the facilities. 
4.3.2.20.1 333 Building. The primary N Reactor Fuels Fabrication activities were conducted in the 333 Building. The fuel fabrication operation produced $N$ Reactor fuel from 1961 until 1987 when operations were stopped.

This facility used a variety of mechanical, chemical, and electrical processes to convert uranium billets and assorted components into finished fuel assemblies for irradiation at $\mathrm{N}$ Reactor.

Operations and resulting radionuclide air emissions in the 333 Building ceased in January 1987. Emission monitoring equipment was shut down at that time. Process liquid effluents are no longer generated. However, steam condensate water, air conditioning water, and stormwater continue to be discharged from the building by way of the process sewer.

Uranium and ${ }^{99} \mathrm{Tc}$ are the most significant radionuclides in surface contamination at the 333 Building. Operation of the heating and cooling ventilation system results in potential diffuse and fugitive emissions from equipment and personnel access doors. However, on-going surveillance activity within the building indicates that radioactive contamination is not present in readily dispersable form.

4.3.2.20.2 313 Building. The 313 Building (N Fuels Fabrication Support Facility) houses: the uranium laboratory, copper casting, waste-acid treatment facility, extrusion press, engineering development laboratory, training rooms, and staff offices.

The 313 Building was used from 1944 to 1971 for fabricating uranium fuel elements for the eight retired, single-pass reactors in the 100 Areas. The function of the building changed when those reactors ceased operating. The building then was used as an N Fuels Fabrication Support Facility to treat uranium-bearing acid in the waste-acid treatment system. The recovered uranium sludge was recycled to form new uranium billets at Fernald, Ohio.

The waste-acid treatment system is still in a stand-by mode of operation for treating uranium bearing clean-up solutions in preparation for Resource Conservation and Recovery Act (RCRA) closure of the waste-acid treatment system. Routine surveys performed in the building indicate that radioactive contamination is not present in readily dispersable forms. Engineering development laboratory work and continuous monitoring of airborne emissions was terminated in 1991.

Uranium and ${ }^{99} \mathrm{Tc}$ are the potential primary radioactive constituents in diffuse and fugitive emissions from the 313 Building. Operation of the heating and cooling ventilation system results in potential diffuse and fugitive emission release points from equipment and personnel access doors.

4.3.2.20.3 303-K Radioactive Mixed Waste Storage Facility. The 303-K Facility was designed and constructed in 1943. The facility is a concrete-block building with a poured concrete ceiling. Outside, the storage area consists of two concrete pads, two asphalt pads, and a gravel area. The north room of the 303-K Facility originally had one roof 
exhaust fan. The fan was used from 1953 to 1977 while aluminum spacer and equipment decontaminating was done. The roof vent fan was replaced with a HEPA-filtered exhaust system in 1977, which was used until the fall of 1982 . The exhaust system was only urned on at the end of the curing operation for the concreted billets of recyclable scrap uranium chips and fines, or if hydrogen levels indicated a billet fire had occurred. The HEPA exhaust system has not operated since the concrete curing operation was discontinued in 1982.

The facility no longer discharges either air emissions or liquid effluents to the environment. It has been used to store radioactive and mixed waste since 1982 . The radioactive materials stored at the facility are in sealed containers and are not readily dispersable.

Uranium and ${ }^{99} \mathrm{Tc}$ are the primary radioactive constituents in potential diffuse and fugitive emissions from the $303-\mathrm{K}$ Facility. Operation of the heating and cooling ventilation system results in potential diffuse and fugitive emissions from equipment and personnel access doors.

4.3.2.20.4 303-M Uranium Oxide Facility. The 303-M Uranium Oxide Facility is adjacent to the 333 Building and consists of one building, an adjacent outdoor drum storage area, and a small process sewer filter building. The 303-M Uranium Oxide Facility stored and treated recycled material generated during the $\mathrm{N}$ Reactor fuel fabrication processes. The fuel fabrication waste material consisted of pyrophoric saw fines and lathe turnings, known as chips, composed of slightly enriched uranium and Zircaloy-2. The wastes were calcinated to remove their pyrophoric properties, eliminating the possibility of spontaneous combustion during transportation.

The 303-M Uranium Oxide Facility operated from 1983 to 1987 for the calcination of wastes generated by WHC and previous operators. The facility ceased operating in 1987.

During operation, the facility operated under negative pressure and the building exhaust was released to the atmosphere through a single stack. The emissions were controlled with a bag-house and HEPA filters.

The 303-M Building is currently closed. The water and electricity have been shut off and the exhaust stack capped. The primary radionuclides associated with the facility are uranium and ${ }^{99} \mathrm{Tc}$.

4.3.2.20.5 304 Uranium Concretion Facility. The 304 Building was designed and constructed in 1952 as a pilot plant for the lead-dip canning process. The 304 's uranium concretion equipment was installed in 1971 with a drainage trench and sump to remove liquids resulting from spills, leaks, and daily operations. Standard spill-response procedures inside the building included washing the spilled waste to the sump where the fines would settle out. The waste water was drained into the 300 Area process sewer, and the fines were 

shoveled from the sump and concreted. Additionally, an outside storage area is on the north side of the facility. The storage area is a concrete pad surrounded by asphalt.

The 304 Building is currently in transition to closure under the Resource Conservation and Recovery Act (RCRA). The building has no inlet air supply system, the water is shut off, and drains have been plugged. The exhaust stack was capped in 1994. Uranium and ${ }^{99} \mathrm{Tc}$ are the primary radionuclides potentially present in diffuse and fugitive emissions from personnel and equipment access doors.

4.3.2.20.6 311 Tank Farm. The recoverable and nonrecoverable uranium-bearing neutralized waste solutions from the 313 Building were combined in aboveground tanks (tanks 40 and 50) in the 311 Tank Farm. These liquid wastes eventually were transferred to tanker trucks and disposed of in the 183-H Basins or the 200 Areas. After 1988, if the wastes were below radioactive release limits, they were shipped to offsite contractors for treatment, storage, and/or disposal. The two tanks received approximately $420,000 \mathrm{gal}$ (1.6 million $\mathrm{L}$ ) of waste each year, during periods of peak production.

In addition to waste tanks 40 and 50, the 311 Tank Farm has a 4,000-gal (15,000-L) tank that was used to store nitric acid and two $10,000-\mathrm{gal}(38,000-\mathrm{L})$ tanks that were used to store sodium hydroxide. The tanks were emptied in 1991 and no longer contain nitric acid or sodium hydroxide.

Uranium and ${ }^{99} \mathrm{Tc}$ are the potential primary radioactive constituents in diffuse and fugitive emissions from the 311 Tank Farm. Diffuse and fugitive emissions may be released by way of uranium-bearing material transfers and unplanned releases.

4.3.2.20.7 334-A Waste Acid Storage Building. The 334-A Waste Acid Storage Building was completed in late 1974 and placed in use in January 1975. For four months in 1973, an underground tank and tank 4 in the 334 Tank Farm were used to collect acid waste that would be transferred to the 313 Building for neutralization. The underground tank began to leak in August 1973 and was removed during construction of the 334-A Building.

The waste acids were discharged directly into the process sewer until the 334-A Building was built in December 1974. The tanks in the 334-A building received approximately $210,000 \mathrm{gal}(790,000 \mathrm{~L})$ of waste acids per year. These waste acids consisted of hydrofluoric, nitric, and sulfuric acids with copper, zirconium, chromium, and uranium in solution. Following storage, the acids were pumped from the 334-A Building to the south end of the 313 Building for neutralization.

The 334-A Building is currently shut down and scheduled for RCRA closure. The building heating and cooling air handling systems have been shut down, the water and electricity have been shut off, and drains have been plugged. The storage tanks in the building have been isolated and cleaned. Uranium and ${ }^{99} \mathrm{Tc}$ are the potential primary radioactive constituent in diffuse and fugitive emissions from the 334-A Building. The potential diffuse and fugitive emission release points include personnel and equipment access doors. 
4.3.2.20.8 340-A Building. The 340-A Building houses six above ground storage tanks which provide temporary storage of radioactive liquid waste. The 340-NT-EX Stack powered exhaust system provides airborne ventilation to the storage tanks, and the 340-A Building air is passively ventilated to the atmosphere via a roof air vent.

Smearable radiological contamination resulting from leakage of radioactive liquid waste from the storage tanks has been detected in the 340-A Building. The radionuclides with the most significant dosimetric impact include ${ }^{90} \mathrm{Sr},{ }^{137} \mathrm{Cs}$, ${ }^{238} \mathrm{Pu},{ }^{239 / 240} \mathrm{Pu}$, and ${ }^{241} \mathrm{Am}$, which could potentially be present in diffuse and fugitive emissions from the facility.

4.3.2.20.9 340-B East Building. The 340-B East Building serves as a railroad car loadout facility. This facility houses railroad cars during the transfer of radioactive liquid wastes from the 340 tanks to the railroad cars.

During periods of time when the railroad cars are not housed in the 340-B East Building, the facility's exhaust system is not operated, thus the building is not maintained at negative pressure. Consequently, diffuse and fugitive emissions can potentially occur. Potential diffuse and fugitive emission release points from the 340-B East Building include personnel and equipment access doors. The radionuclides with the largest dosimetric impact that could be potentially released as diffuse and fugitive emissions include: ${ }^{90} \mathrm{Sr},{ }^{137} \mathrm{Cs},{ }^{238} \mathrm{Pu}$, ${ }^{239 / 240} \mathrm{Pu}$, and ${ }^{241} \mathrm{Am}$.

4.3.2.21 Fast Flux Test Facility (FFTF). The FFTF is a 400-megawatt-thermal, sodium-cooled, fast-neutron-flux reactor designed specifically for irradiation testing of nuclear reactor fuels and materials for liquid-metal, fast-breeder reactors. The reactor is capable of extensive in-core irradiation testing, including eight core positions that may be used with independent instrumentation for the test loops. In addition to irradiation-testing capabilities, FFTF is designed for long-term testing and evaluation of plant components and systems for liquid-metal, fast-breeder reactors.

The FFTF is a complex of buildings and equipment arranged around the reactor containment building. This arrangement includes the reactor, equipment, and structures for heat removal, containment, core component handling and examination, instrumentation and control, and buildings for supplying utilities and other essential services. The reactor is currently shut down.

Radionuclides primarily associated with FFTF include ${ }^{41} \mathrm{Ar},{ }^{85} \mathrm{Kr},{ }^{90} \mathrm{Sr},{ }^{131} \mathrm{I}$, and ${ }^{137} \mathrm{Cs}$. The potential primary diffuse and fugitive emission release points include the personnel and equipment access doors and the inlet-exhaust ventilation system, all of which are controlled. 


\subsection{SUPPLEMENTAL INFORMATION}

This section has supplemental information related to Hanford Site radionuclide air emissions in 1995. The information consists of:

- Population dose estimate

- Compliance status with Subparts Q and T of 40 CFR 61 (EPA 1992a, EPA 1992b)

- Discussion on ${ }^{220} \mathrm{Rn}$ emissions

- Emission points subject to monitoring requirements of 40 CFR 61.93(b) and costs to upgrade noncompliant monitoring systems

- Emissions estimates and confirmatory measurement data for specific NOCs

- Status of quality assurance (QA) program intended to comply with description in 40 CFR 61, Appendix B, Method 114.

\subsection{POPULATION DOSE}

The regional population dose (collective EDE) from 1995 Hanford Site air emissions was estimated by calculating the radiation dose to the population of approximately 376,000 residing within an $80-\mathrm{km}(50-\mathrm{mi})$ radius of the onsite operating areas, (PNL 1991).

Pathways of exposure to the population from releases of radionuclides to the atmosphere include inhalation, air submersion, ground-shine, and consumption of food. Population exposure to radionuclide air emissions was determined using values of population-weighted atmospheric dispersion factors for distance and each compass sector. The collective EDE (using the GENII computer code, [PNL 1988]) from air emissions to the population within $80-\mathrm{km}$ of the Hanford Site during 1995 was 0.0016 person-Sv (0.16 person-rem). Releases to surface water added another 0.0016 person-Sv ( 0.16 person-rem) so the population dose from all Hanford Site radionuclide air and liquid effluents was 0.0033 person-Sv $(0.33$ person-rem) in 1995.

\subsection{COMPLIANCE STATUS WITH SUBPARTS Q AND T OF 40 CFR 61}

In 40 CFR 61, Subpart Q (EPA 1992a), "National Emission Standards for Radon Facilities From Department of Energy Facilities, " paragraph 61.190 states that the provisions of Subpart Q apply to the design and operation of all storage and disposal facilities for radium-bearing material that emit ${ }^{222} \mathrm{Rn}$ to the air. Paragraph 61.191 (b) states that a source means any building, structure, pile, impoundment, or area used for interim storage or 
disposal that is or contains waste material containing radium in sufficient concentration to emit ${ }^{222} \mathrm{Rn}$ in excess of the $20 \mathrm{pCi} / \mathrm{m}^{2} / \mathrm{s}$. At the Hanford Site, no storage and disposal facilities for radium-bearing waste materials are established. Therefore, the provisions of this subpart are not applicable to the Hanford Site.

Activities at the Hanford Site were evaluated for compliance with 40 CFR 61 Subpart T, "National Emissions Standards for Radon Emissions From the Disposal of Uranium Mill Tailings" (EPA 1992b). In paragraph 61.220, "Designation of Facilities," owners and operators of all sites used for the disposal of tailings and that managed residual radioactive material or uranium byproduct materials during and following the processing of uranium ores and that are listed in or designated by the Secretary of Energy under Title I of the Uranium Mill Tailings Control Act of 1978 or regulated under Title II of that act are subject to the provisions of the subpart. At the Hanford Site, uranium milling and uranium-ore processing activities are not conducted. Therefore, the provisions of this subpart are not applicable to the Hanford Site.

\subsection{EMISSION OF RADON-220 FROM SOURCES CONTAINING THORIUM-228}

Radon-220 is a gas that passes unimpeded through the HEPA filters and sample filters. At the 327 Building, emissions of ${ }^{220} \mathrm{Rn}$ were directly measured with a continuous air monitor.

Radon emissions and doses for 1995 are being reported separately from other point source emissions because 40 CFR 61 Subpart $\mathrm{H}$ excludes reporting of radon emissions from DOE facilities. The ${ }^{220} \mathrm{Rn}$ released from the 327 Building originated from a ${ }^{228} \mathrm{Th}$ source. Releases from this facility are listed in Table $2-1$. The resulting ${ }^{220} \mathrm{Rn}$ dose was 0.0033 mrem to the maximally exposed individual at the Sagemore Road farm, see Table 3-3.

The calculation was performed assuming that all ${ }^{220} \mathrm{Rn}$ was converted to its first long-lived decay product, ${ }^{212} \mathrm{~Pb}$, before reaching the receptor. This was necessary because CAP88 does not account for ingrowth of decay products for the short-lived ${ }^{220} \mathrm{Rn}$ radionuclide. The ${ }^{222} \mathrm{Rn}$ emissions from the 327 Building added another $1.9 \mathrm{E}-04 \mathrm{mrem}$ to the MEI's dose. Because of the ${ }^{220} \mathrm{Rn}$ and ${ }^{222} \mathrm{Rn}$ emissions, during 1995 , the dose from these radionuclides was roughly equivalent to the dose to the $\mathrm{MEI}$ from all other radionuclides. The total dose to the MEI at Sagemore from all air emissions and radionuclides, including ${ }^{220} \mathrm{Rn}$ and ${ }^{222} \mathrm{Rn}$, was $0.027 \mathrm{mrem}$, which was less than $0.3 \%$ of the $10 \mathrm{mrem} / \mathrm{year}$ regulatory standard. 


\subsection{SOURCES OF RADON-222}

Radon-222 is a decay product in the ${ }^{238} \mathrm{U}$ decay chain. The contribution of ${ }^{222} \mathrm{Rn}$ to the dose received by any member of the public can be calculated after removing the background uranium activity from soil analytical data. The radon flux that could be derived from any residual or above-background radioactivity, as a measure of the Hanford Site component, appears to be extremely small and would result in an offsite dose much smaller than $0.1 \mathrm{mrem} / \mathrm{y} \mathrm{EDE}$.

Surface-soil samples were collected at locations both on and off the Hanford Site. The purpose of this sampling is to detect the possible buildup of radionuclides deposited by air emissions from Hanford Site facilities. Samples are collected at relatively undisturbed nonagricultural sites so that natural deposition and buildup processes would be represented. Because the radionuclides of interest were present in worldwide fallout or occurred both naturally and in Hanford Site emissions, these radionuclides were expected in all samples.

An assessment of radionuclide contributions from Hanford Site operations was made by comparing results from samples collected: (1) on the Hanford Site with those collected off the Hanford Site, (2) around the Hanford Site perimeter with those collected at distant locations, and (3) downwind (primarily east and south of the Hanford Site) with those collected from generally upwind and distant locations. Analytical results obtained from each location in 1995 were compared to results obtained from the same location in previous years. The comparison revealed no indication of trends or increases in the concentrations of radionuclides in the offsite environment that could be attributed to Hanford Site activities.

Because the onsite concentrations of these radionuclides are not significantly different from the offsite concentrations, there does not appear to have be a long-term buildup of radon decay products in the soil resulting from unmonitored ${ }^{222} \mathrm{Rn}$ emissions from the Hanford Site. The only measured radon release was from the 327 Building which was reported to be $0.4 \mathrm{Ci}$ of ${ }^{222} \mathrm{Rn}$, resulting in an EDE to the $\mathrm{MEI}$ of $1.9 \mathrm{E}-04 \mathrm{mrem}$ (see Table 3-3).

\subsection{EMISSION POINTS SUBJECT TO MONITORING REQUIREMENTS OF 40 CFR 61.93(b) AND THE COSTS TO UPGRADE NONCOMPLIANT MONITORING SYSTEMS}

All Hanford Site powered ventilated point sources with the potential to emit radionuclides were assessed and compared with the threshold limit of $0.1 \mathrm{mrem} / \mathrm{y}$ in $40 \mathrm{CFR}$ 61 Subpart H (EPA 1988). As of 1995 all 121 point sources with potential to emit radionuclides were assessed for the EDE to the nearest receptor from potential radionuclide emissions (i.e. emissions assuming no control devices in place). Of these point sources, 25 were identified as major point sources (i.e. point sources with potential emissions causing an EDE to the MEI greater than $0.1 \mathrm{mrem} / \mathrm{y})(40 \mathrm{CFR} 61.93(\mathrm{~b})(4)(\mathrm{i})$ ). WHC manages 20 of the major point sources while PNNL manages five. All of these major point sources, listed 
below, are covered by a Federal Facility Compliance Agreement signed by DOE RL and EPA, Region 10 to bring their point source sampling systems into compliance.

- 200 East Area

- 291-A-1 (PUREX; WHC)

- 291-B-1 (B Plant; WHC)

- 296-A-1 (PUREX; WHC)

- 296-A-12 (East Tank Farms; WHC)

- 296-A-17/296-P-26 (East Tank Farms; WHC)

- 296-A-22 (242-A Evaporator; WHC)

- 296-A-25 (East Tank Farms; WHC)

- 296-A-40 (East Tank Farms; WHC)

- 296-B-28 (East Tank Farms; WHC)

- 296-C-05 (East Tank Farms; WHC)

- 296-P-16 (East Tank Farms; WHC)

- 296-P-32, 33, and 34 (Tank Farms Characterization Project; WHC)

- 200 West Area

- 291-Z-1 (PFP; WHC)

- 296-S-15 (West Tank Farms; WHC)

- 296-S-22 (West Tank Farms; WHC)

- 296-T-18 (West Tank Farms; WHC)

- 300 Area

- 340-NT-EX (340 Waste Handling; WHC)

- EP-324-01-S (324 Building; PNNL)

- EP-325-01-S (325 Building; PNNL)

- EP-327-01-S (327 Building; PNNL)

- EP-327-02-V (327 Decontamination Cell; PNNL)

- EP-3720-01-S (3720 Building; PNNL).

The Pacific Northwest National Laboratory's five major point source sampling systems were brought into compliance by February 1994. Of the twenty major point sources managed by WHC six point source sampling systems have been brought into compliance by the end of 1995, four new point sources were already in compliance. Schedules have been developed for the remaining ten point sources to bring their sampling systems into compliance. The cost estimate to bring the remaining sampling systems into compliance is approximately $\$ 14$ million. 


\subsection{EMISSIONS ESTIMATES AND CONFIRMATORY MEASUREMENT DATA FOR SPECIFIC NOCs}

This section contains the emissions estimates and periodic confirmatory measurement data as required by specific Notices of Construction (NOCs) and other regulatory agreements.

\subsubsection{NOC for Portable Temporary Radioactive Airborne Emissions Units (PTRAEU)}

The NOC for PTRAEUs requires that the emissions from each unit be summarized in the annual Radionuclide Air Emissions Report for the Hanford Site. The information provided in Table 5-1 fulfills this requirement.

Table 5-1

\begin{tabular}{|c|c|c|c|c|c|}
\hline \multicolumn{6}{|c|}{ CY-1995 Emissions Estimates for PTREAUs ${ }^{a}$} \\
\hline $\begin{array}{l}\text { Unit } \\
\text { Type }\end{array}$ & $\begin{array}{l}\text { Identification } \\
\text { Number }\end{array}$ & $\begin{array}{l}\text { CY-1995 } \\
\text { Operating } \\
\text { Hours }\end{array}$ & $\begin{array}{l}\text { Discharge } \\
\text { Volume } \\
\left(\mathbf{m}^{3}\right)\end{array}$ & $\begin{array}{l}\text { Radionuclide } \\
\text { or Type of } \\
\text { Radioactivity }\end{array}$ & $\begin{array}{l}\text { Annual } \\
\text { Estimated } \\
\text { Emissions } \\
\text { (Ci) }\end{array}$ \\
\hline \multicolumn{6}{|c|}{ Environmental Restoration Operations (N Reactor) } \\
\hline \multirow{4}{*}{$\begin{array}{l}\text { General Dynamics } \\
\text { Model SP-500 } \\
\text { HEPA Filtration Module (Type I) }\end{array}$} & SN1103 & 0.0 & $0.0 \mathrm{E}+00$ & $\mathrm{~N} / \mathrm{A}$ & $\mathrm{N} / \mathrm{A}$ \\
\hline & SN1699 & 0.0 & $0.0 \mathrm{E}+00$ & N/A & N/A \\
\hline & SN1097 & 0.0 & $0.0 \mathrm{E}+00$ & N/A & N/A \\
\hline & SN 1101 & 0.0 & $0.0 \mathrm{E}+\infty 0$ & $\mathrm{~N} / \mathrm{A}$ & $\mathrm{N} / \mathrm{A}$ \\
\hline $\begin{array}{l}\text { General Dynamics } \\
\text { Model PFB(H)-1000 HEPA Filtration } \\
\text { Module (Type 1) }\end{array}$ & SN1079 & 0.0 & $0.0 \mathrm{E}+00$ & $\overline{\mathrm{N} / \mathrm{A}}$ & N/A \\
\hline $\begin{array}{l}\text { Control Resources Systems Inc. } \\
\text { Model CRSI-2000 (Type I) }\end{array}$ & SN710-2416 & 0.0 & $0.0 \mathrm{E}+00$ & N/A & $\mathrm{N} / \mathrm{A}$ \\
\hline \multirow{5}{*}{$\begin{array}{l}\text { Euroclean HEPA Vacs } \\
\text { Model UZ930 (Type I) }\end{array}$} & SN1360114 & 0.0 & $0.0 \mathrm{E}+00$ & $\mathrm{~N} / \mathrm{A}$ & N/A \\
\hline & SN2040064 & 0.0 & $0.0 \bar{E}+00$ & N/A & N/A \\
\hline & SN2040067 & 0.0 & $0.0 \mathrm{E}+00$ & N/A & $\overline{N / A}$ \\
\hline & SN2040095 & 0.0 & $0.0 \mathrm{E}+00$ & N/A & N/A \\
\hline & SN13500064 & 0.0 & $0.0 \mathrm{E}+00$ & N/A & N/A \\
\hline \multirow{2}{*}{$\begin{array}{l}\text { Euroclean HEPA Vacs } \\
\text { Model UZ948 (Type I) }\end{array}$} & $\mathrm{SN} 1520 \mathrm{U}$ & 0.0 & $0.0 \mathrm{E}+00$ & N/A & $N / A$ \\
\hline & SN918009 & 0.0 & $0.0 \mathrm{E}+00$ & N/A & N/A \\
\hline \multicolumn{6}{|c|}{ Environmental Restoration Operations } \\
\hline NPO E1000PC (Type I) & WB34147 & 0.0 & $0.0 \mathrm{E}+00$ & N/A & N/A \\
\hline \multirow[t]{4}{*}{ Blue Max Optima 2000 (Type I) } & 0050979 & 0.0 & $0.0 \mathrm{E}+00$ & N/A & N/A \\
\hline & 0042772 & 0.0 & $0.0 \mathrm{E}+00$ & N/A & N/A \\
\hline & 0030661 & 0.0 & $0.0 \mathrm{E}+00$ & $\mathrm{~N} / \mathrm{A}$ & N/A \\
\hline & 0030559 & 0.0 & $0.0 \mathrm{E}+00$ & N/A & N/A \\
\hline
\end{tabular}


Table 5-1

\section{CY-1995 Emissions Estimates for PTREAUs ${ }^{a}$}

\begin{tabular}{|c|c|c|c|c|c|}
\hline $\begin{array}{l}\text { Unit } \\
\text { Type }\end{array}$ & $\begin{array}{l}\text { Identification } \\
\text { Number }\end{array}$ & $\begin{array}{l}\text { CY-1995 } \\
\text { Operating } \\
\text { Hours }\end{array}$ & $\begin{array}{l}\text { Discharge } \\
\text { Volume } \\
\left(\mathbf{m}^{3}\right)\end{array}$ & $\begin{array}{l}\text { Radionuclide } \\
\text { or Type of } \\
\text { Radioactivity }\end{array}$ & $\begin{array}{l}\text { Annual } \\
\text { Estimated } \\
\text { Emissions } \\
\quad \text { (Ci) }\end{array}$ \\
\hline $\begin{array}{l}\text { Flanders Ventilation Unit } \\
\text { (Type I) }\end{array}$ & WBS2452 & 0.0 & $0.0 \mathrm{E}+00$ & N/A & N/A \\
\hline $\begin{array}{l}\text { Kelly Decontamination System } \\
\text { (Type I) }\end{array}$ & WB56653 & 0.0 & $0.0 \mathrm{E}+00$ & N/A & $\mathrm{N} / \mathrm{A}$ \\
\hline Exhauster (Type I) & WC16133 & 0.0 & $0.0 \mathrm{E}+00$ & N/A & $\mathrm{N} / \mathrm{A}$ \\
\hline \multicolumn{6}{|c|}{ K Basin Operations } \\
\hline \multirow{2}{*}{$\begin{array}{l}\text { Blue Max Optima } 2000 \\
\text { (Type I) }\end{array}$} & SN0050878 & 0.0 & $0.0 \mathrm{E}+00$ & $\mathrm{~N} / \mathrm{A}$ & $\mathrm{N} / \mathrm{A}$ \\
\hline & SN0030560 & 0.0 & $0.0 \mathrm{E}+\infty 0$ & $\mathrm{~N} / \mathrm{A}$ & $\mathrm{N} / \mathrm{A}$ \\
\hline \multirow{4}{*}{$\begin{array}{l}\text { NFS/RPS } \\
\text { Model SP-125A HEPA Filtration } \\
\text { Unit }\end{array}$} & 1161 & 0.0 & $0.0 \mathrm{E}+00$ & N/A & N/A \\
\hline & 1162 & 0.0 & $0.0 \mathrm{E}+00$ & $\mathrm{~N} / \mathrm{A}$ & N/A \\
\hline & 1163 & 0.0 & $0.0 \mathrm{E}+00$ & $\mathrm{~N} / \mathrm{A}$ & $\mathrm{N} / \mathrm{A}$ \\
\hline & 1164 & 0.0 & $0.0 \mathrm{E}+00$ & $\mathrm{~N} / \mathrm{A}$ & N/A \\
\hline $\begin{array}{l}\text { NFS/RPS } \\
\text { Model SP-505A HEPA Filtration } \\
\text { Unit }\end{array}$ & 1170 & 0.0 & $0.0 \mathrm{E}+00$ & $\overline{\mathrm{N} / \mathrm{A}}$ & N/A \\
\hline \multicolumn{6}{|c|}{ B Plant Environmental Engineering } \\
\hline \multirow[t]{2}{*}{ Micro-Trap (Type I) } & $21303006 \# 1$ & 0.0 & $0.0 \mathrm{E}+00$ & N/A & N/A \\
\hline & 2B03007 \#2 & 0.0 & $0.0 \mathrm{E}+00$ & $\mathrm{~N} / \mathrm{A}$ & N/A \\
\hline $\begin{array}{l}\text { Euroclean } \\
\text { Model UZ878A (Type I) }\end{array}$ & $9042015-020$ & 0.0 & $0.0 \mathrm{E}+00$ & $\mathrm{~N} / \mathrm{A}$ & N/A \\
\hline Vacuum Loader ("Guzzler") & $\begin{array}{l}\text { G2411WAZ4- } \\
1162 \mathrm{TC} 94\end{array}$ & 0.0 & $0.0 \mathrm{E}+00$ & $\mathrm{~N} / \mathrm{A}$ & N/A \\
\hline \multicolumn{6}{|c|}{300 Area Support Service } \\
\hline \multirow[t]{2}{*}{ Euroclean (Type I) } & 9470005 & 0.0 & $0.0 \mathrm{E}+00$ & N/A & N/A \\
\hline & 9500081 & 0.0 & $0.0 \mathrm{E}+00$ & N/A & N/A \\
\hline Phantom II (Type I) & 13296 & 0.0 & $0.0 \mathrm{E}+00$ & $\mathrm{~N} / \mathrm{A}$ & N/A \\
\hline Micro-Trap Jr. (Type I) & MT-207 & 0.0 & $0.0 \mathrm{E}+00$ & N/A & $\mathrm{N} / \mathrm{A}$ \\
\hline Micro-Trap & 12632 & 223 & $1.5 \mathrm{E}+06$ & $\begin{array}{l}\text { total alpha } \\
\text { tocal beta }\end{array}$ & $\begin{array}{l}\text { ND } \\
\text { ND }\end{array}$ \\
\hline \multicolumn{6}{|c|}{ PUREX Regulatory Compliance } \\
\hline Micro-Trap (Type 1) & MT-201 & 0.0 & $0.0 \mathrm{E}+00$ & N/A & N/A \\
\hline \multirow[t]{2}{*}{ Micro-Trap Jr. (Type I) } & 04167 & 0.0 & $0.0 \mathrm{E}+\infty 0$ & N/A & N/A \\
\hline & MT- 12727 & 0.0 & $0.0 \mathrm{E}+00$ & N/A & $\mathrm{N} / \mathrm{A}$ \\
\hline Kelly Decontamination Unit (Type I) & WB56633 & 0.0 & $0.0 \mathrm{E}+00$ & N/A & N/A \\
\hline Aero-Clear 2000 (Type I) & $9900-2000-0845$ & 0.0 & $0.0 \mathrm{E}+00$ & N/A & N/A \\
\hline
\end{tabular}


Table 5-1

(3 sheets)

\begin{tabular}{|c|c|c|c|c|c|}
\hline \multicolumn{6}{|c|}{ CY-1995 Emissions Estimates for PTREAUs ${ }^{a}$} \\
\hline $\begin{array}{l}\text { Unit } \\
\text { Type }\end{array}$ & $\begin{array}{l}\text { Identification } \\
\text { Number }\end{array}$ & $\begin{array}{l}\text { CY-1995 } \\
\text { Operating } \\
\text { Hours }\end{array}$ & $\begin{array}{c}\text { Discharge } \\
\text { Volume } \\
\left(\mathbf{m}^{3}\right)\end{array}$ & $\begin{array}{l}\text { Radionuclide } \\
\text { or Type of } \\
\text { Radioactivity }\end{array}$ & $\begin{array}{c}\text { Annual } \\
\text { Estimated } \\
\text { Emissions } \\
\text { (Ci) }\end{array}$ \\
\hline \multicolumn{6}{|c|}{ Mobile Sample Screening and Analysis Facilities } \\
\hline \multirow[t]{3}{*}{ Sampling Lab (Type III) } & 64.5189 & 0.0 & $0.0 \mathrm{E}+00$ & $\mathrm{~N} / \mathrm{A}$ & $\mathrm{N} / \mathrm{A}$ \\
\hline & $64-4276$ & 0.0 & $0.0 \mathrm{E}+00$ & $\mathrm{~N} / \mathrm{A}$ & $\mathrm{N} / \mathrm{A}$ \\
\hline & $64-4230$ & 0.0 & $0.0 \mathrm{E}+00$ & N/A & $\mathrm{N} / \mathrm{A}$ \\
\hline \multirow[t]{7}{*}{ Sample Preparation (Type II) } & $64-4228$ & 3.5 & TBD & TBD & TBD \\
\hline & $64-4229$ & 12.84 & TBD & $\widehat{T B D}$ & TBD \\
\hline & $64-4231$ & $\overline{0.0}$ & $0.0 \mathrm{E}+00$ & N/A & N/A \\
\hline & $68 \mathrm{~B} 4625$ & 0.0 & $0.0 \mathrm{E}+00$ & N/A & $\mathrm{N} / \mathrm{A}$ \\
\hline & $68 \mathrm{~B} 4626$ & 0.0 & $0.0 \mathrm{E}+00$ & $\mathrm{~N} / \mathrm{A}$ & N/A \\
\hline & $68 \mathrm{~B} 4630$ & $\overline{0.0}$ & $0.0 \mathrm{E}+00$ & $\mathrm{~N} / \mathrm{A}$ & $\mathrm{N} / \mathrm{A}$ \\
\hline & $68 \mathrm{~B} 4631$ & 5.83 & TBD & TBD & TBD \\
\hline \multicolumn{6}{|c|}{ 222-S Laboratory } \\
\hline Air Cannon (Type I) & $1947 \mathrm{~K} 31$ & 0.0 & $0.0 \mathrm{E}+00$ & N/A & $\mathrm{N} / \mathrm{A}$ \\
\hline \multirow[t]{2}{*}{ Blue Max (Type I) } & SN022049 & 0.0 & $0.0 \mathrm{E}+00$ & N/A & N/A \\
\hline & SN0011721 & 0.0 & $0.0 \mathrm{E}+00$ & $\mathrm{~N} / \mathrm{A}$ & N/A \\
\hline
\end{tabular}

Notes:

a $\quad$ N/A $=$ Not Applicable, indicates that the unit did not operate; ND $=$ None Detected; TBD $=$ To Be Determined, emissions estimates were not available at the time of preparing this report, but will be provided to U.S. EPA Region 10 and WDOH by July $31,1996$. 


\subsubsection{NOC for the Environmental Analytical Laboratory (EAL)}

The NOC for the EAL requires that the emissions be estimated and included in the annual Radionuclide Air Emissions Report for the Hanford Site. The information provided in Table 5-2 fulfills this requirement.

Table 5-2

\begin{tabular}{|c|c|l|c|}
\hline \multicolumn{3}{|c|}{ CY-1995 Emissions Estimates } \\
for the \\
Environmental Analytical Laboratory \\
\hline $\begin{array}{c}\text { Emission Point } \\
\text { 1dentification } \\
\text { Number }\end{array}$ & $\begin{array}{c}\text { Discharge } \\
\text { Volume } \\
\left(\mathbf{m}^{3}\right)\end{array}$ & $\begin{array}{c}\text { Radionuclide } \\
\text { or Type of } \\
\text { Radioactivity }\end{array}$ & $\begin{array}{c}\text { Annual } \\
\text { Estimated } \\
\text { Emissions } \\
\text { (Ci) }\end{array}$ \\
\hline HO-64-4230 & $1.8 \mathrm{E}+05$ & $\begin{array}{l}\text { total alpha } \\
\text { total beta }\end{array}$ & $\begin{array}{c}8.1 \mathrm{E}-11 \\
1.7 \mathrm{E}-09\end{array}$ \\
\hline HO-64-4276 & $1.5 \mathrm{E}+05$ & $\begin{array}{l}\text { total alpha } \\
\text { total beta }\end{array}$ & $\begin{array}{c}2.1 \mathrm{E}-10 \\
1.7 \mathrm{E}-09\end{array}$ \\
\hline
\end{tabular}

\subsubsection{Release Estimates for the L-070 Project}

The L-070 Project is the activity to upgrade the 300 Area Process Sewer and Retention Process Sewer piping system. Estimates of the 1995 radioactive emissions, associated with the L-070 Project, are provided in Table 5-4.

Table 5-4

\begin{tabular}{|c|c|}
\hline \multicolumn{2}{|c|}{$\begin{array}{c}\text { CY-1995 Emissions Estimates } \\
\text { for the } \\
\text { L-070 Project }\end{array}$} \\
\hline $\begin{array}{c}\text { Radionuclide } \\
\text { or Type of } \\
\text { Radioactivity }\end{array}$ & $\begin{array}{c}\text { Annual } \\
\text { Estimated } \\
\text { Emissions } \\
\text { (Ci) }\end{array}$ \\
\hline $\begin{array}{l}\text { total alpha } \\
\text { total beta }\end{array}$ & $4.1 \mathrm{E}-05$ \\
\hline & $4.3 \mathrm{E}-05$ \\
\hline
\end{tabular}




\subsection{STATUS OF COMPLIANCE WITH QA PROGRAM CRITERIA DESCRIBED IN APPENDIX B, METHOD 114, 40 CFR 61}

The document Quality Assurance Program for Radionuclide Airborne Emissions Monitoring (WHC 1995c) contain appendices that provide point-by-point comparisons of the major point source monitoring systems to the quality assurance criteria of 40 CFR 61 , Appendix B Method 114 (EPA 1992). The document Effluent Monitoring Quality Assurance Project Plan for Radioactive Airborne Emissions Data (WHC 1995a) contains the quality assurance activities associated with data collection and reporting. Westinghouse Hanford Company and Bechtel Hanford Incorporated monitoring activities are conducted in compliance with state and federal requirements provided in these quality assurance documents. Both documents are updated annually and submitted to the EPA and WDOH.

Pacific Northwest National Laboratory has implemented the Quality Assurance Plan for Facility Effluent Monitoring, Quality Assurance Plan No. F0-011. The QA program described by this plan is based on the following documentation:

- $\quad$ EPA QAMS-005/80, Interim Guidelines for Preparing Quality Assurance Project Plans

- DOE 5700.6C, Quality Assurance

- DOE 5400.1, General Environmental Protection Program

- $\quad$ DOE/EH-0173T, Environmental Regulatory Guide for Radiological Effluent Monitoring and Environmental Surveillance

- applicable criteria of ASME NQA-1, Quality Assurance Requirements for Nuclear Facilities, as reflected in PNNL's Quality Assurance Manual, PNL-MA-70, and associated implementing procedures. 
DOE/RL-96-37

This page intentionally left blank. 


\subsection{REFERENCES}

Brown, R. M., 1990, Oxidation and Dispersion of HT in the Environment: The August 1986 Field Experiment at Chalk River, Health Physics 58(2):171-181.

DOE, 1988, General Environmental Protection Program, DOE Order 5400.1, U.S. Department of Energy, Washington, D.C.

DOE, 1991, Environmental Regulatory Guide for Radiological Effluent Monitoring and Environmental Surveillance, DOE/EH-0173T, U.S. Department of Energy, Washington D.C.

DOE-RL, 1988, Hanford Site Dangerous Waste Part A Permit Application, DOE/RL-88-21, U.S. Department of Energy, Richland Operations Office, Richland, Washington.

DOE-RL, 1991, Calendar Year 1990 Air Emissions Report for the Hanford Site, DOE/RL-91-10, U.S. Department of Energy, Richland Field Office, Richland, Washington.

DOE-RL, 1992, Radionuclide Air Emissions Report for the Hanford Site, Calendar Year 1991, DOE/RL-92-30, U.S. Department of Energy, Richland Field Office, Richland, Washington.

DOE-RLa, 1993, Radionuclide Air Emissions Report for the Hanford Site, Calendar Year 1992, DOE/RL-93-36, U.S. Department of Energy, Richland Field Office, Richland, Washington.

DOE-RLb, 1993, Registration for the Hanford Site: Sources of Radioactive Emissions, DOE/RL-84-08-08, U.S. Department of Energy, Richland Field Office, Richland, Washington.

DOE-RL, 1994, Radionuclide Air Emissions Report for the Hanford Site, Calendar Year 1993, DOE/RL-94-51, U.S. Department of Energy, Richland Field Office, Richland, Washington.

DOE-RL, 1995, Radionuclide Air Emissions Report for the Hanford Site, Calendar Year 1994, DOE/RL-95-49, U.S. Department of Energy, Richland Field Office, Richland, Washington.

EPA, 1989, "National Emission Standards for Hazardous Air Pollutants," Subpart H, Title 40, Code of Federal Regulations, Part 61, U.S. Environmental Protection Agency, Washington, D.C. 
EPA, 1992a, "National Emission Standards for Radon Facilities From Department of Energy Facilities", Subpart Q, Title 40, Code of Federal Regulations, Part 61, U.S. Environmental Protection Agency, Washington, D.C.

EPA, 1992ba, "National Emissions Standards for Radon Emissions From the Disposal of Uranium Mill Tailings" , Subpart T, Title 40, Code of Federal Regulations, Part 61, U.S. Environmental Protection Agency, Washington, D.C.

Parks, B. S. 1992. User's Guide for CAP88-PC, Version 1.0, 402-B-92-001, Office of Radiation Programs, U.S. Environmental Protection Agency, Las Vegas, Nevada.

PNL, 1988, GENII - The Hanford Environmental Radiation Dosimetry Software System, PNL-6584, Vols. 1-3, Pacific Northwest Laboratory, Richland, Washington.

PNL, 1990, Hanford Site Environmental Report for Calendar Year 1989, PNL-7346, Pacific Northwest Laboratory, Richland, Washington.

PNL, 1991, Hanford Area 1990 Population and 50-year Projections, PNL-7803, Pacific Northwest Laboratory, Richland, Washington.

PNL, 1993, Recommended Environmental Dose Calculation Methods and Hanford-Specific Parameters, PNL-3777, Rev. 2., Pacific Northwest Laboratory, Richland, Washington.

PNL, 1994a, Hanford Site Environmental Data for Calendar Year 1993 -- Surface and Columbia River, PNL-9824, Pacific Northwest Laboratory, Richland, Washington.

PNL, 1994b, Climatological Data Summary 1993 with Historical Data, PNL-9809, Pacific Northwest Laboratory, Richland, Washington.

PNL, 1995, Hanford Site National Environmental Policy Act (NEPA) Characterization, PNL-6415 Rev. 7, Pacific Northwest Laboratory, Richland, Washington.

PNNL, 1995, Assessment of Unabated Facility Emission Potentials for Evaluating Airborne Radionuclide Monitoring Requirements at Pacific Northwest National Laboratory 1995, PNL-10855, Pacific Northwest National Laboratory, Richland, Washington.

PNNL, 1996a, Hanford Site Environmental Report for Calendar Year 1995, PNNL-11139, Pacific Northwest National Laboratory, Richland, Washington.

PNNL, 1996b, 1995 Surface Environmental Surveillance Data, PNNL-11140, Pacific Northwest National Laboratory, Richland, Washington. 
SNL, 1992, User's Guide for GENII-S: A Code for Statistical and Deterministic Simulations of Radiation Doses to Humans from Radionuclides in the Environment, SAND91-0561A, Sandia National Laboratories, Albuquerque, New Mexico.

Uranium Mill Tailings Radiation Control Act of 1978, Public Law 95-604, 42 USC 7901.

WHC, 1991, Unit Dose Calculation Methods and Summary of Facility Effluent Monitoring Plan Determinations, WHC-EP-0498, Westinghouse Hanford Company, Richland, Washington.

WHC, 1992a, Status of Birds at the Hanford Site in Southeastern Washington, WHC-EP-0402, Rev. 1, Westinghouse Hanford Company, Richland, Washington.

WHC, 1992b, Vascular Plants of the Hanford Site, WHC-EP-0554, Westinghouse Hanford Company, Richland, Washington.

WHC, 1994a, Hanford Site Registered Stack Assessment for Potential Radionuclide Emissions, WHC-SD-EN-RPT-007, Rev. 0, Westinghouse Hanford Company, Richland, Washington.

WHC, 1994b, Hanford Site Radionuclide National Emission Standards for Hazardous Air Pollutants Unregistered Stack (Powered Exhaust) Source Assessment, WHC-SD-EN-RPT-025, Rev. 0, Westinghouse Hanford Company, Richland, Washington.

WHC, 1995a, Effluent Monitoring Quality Assurance Project Plan for Radioactive Airborne Emissions Data, WHC-EP-0528-2, Westinghouse Hanford Company, Richland, Washington.

WHC, 1995b, Hanford Site Radionuclide National Emsission Standards for Hazardous Air Pollutants Registered and Unregistered Stack (Powered Exhaust) Source Assessment, WHC-EP-0894, Westinghouse Hanford Company, Richland, Washington.

WHC, 1995c, Quality Assurance Program Plan for Radionuclide Airborne Emissions Monitoring, WHC-EP-0536-3, Westinghouse Hanford Company, Richland, Washington.

WHC, 1996, Westinghouse Hanford Company Operational Environmental Monitoring Annual Report, Calendar Year 1995, WHC-EP-0573-4, Westinghouse Hanford Company, Richland, Washington.

Zacks, R. S., March 1995, Biological Diversity Inventory and Analysis at the Hanford Site: Insects, Department of Entomology, Washington State University, Pullman, Washington. 


\section{DOE/RL-96-37}

This page intentionally left blank. 
DOE/RL-96-37

APPENDIX

DOSE MODELING AND METEOROLOGICAL DATA 
DOE/RL-96-37

This page intentionally left blank. 
DOE/RL-96-37

Figure A-1. Meteorological Station Map and Wind Roses for Calendar Year 1995.

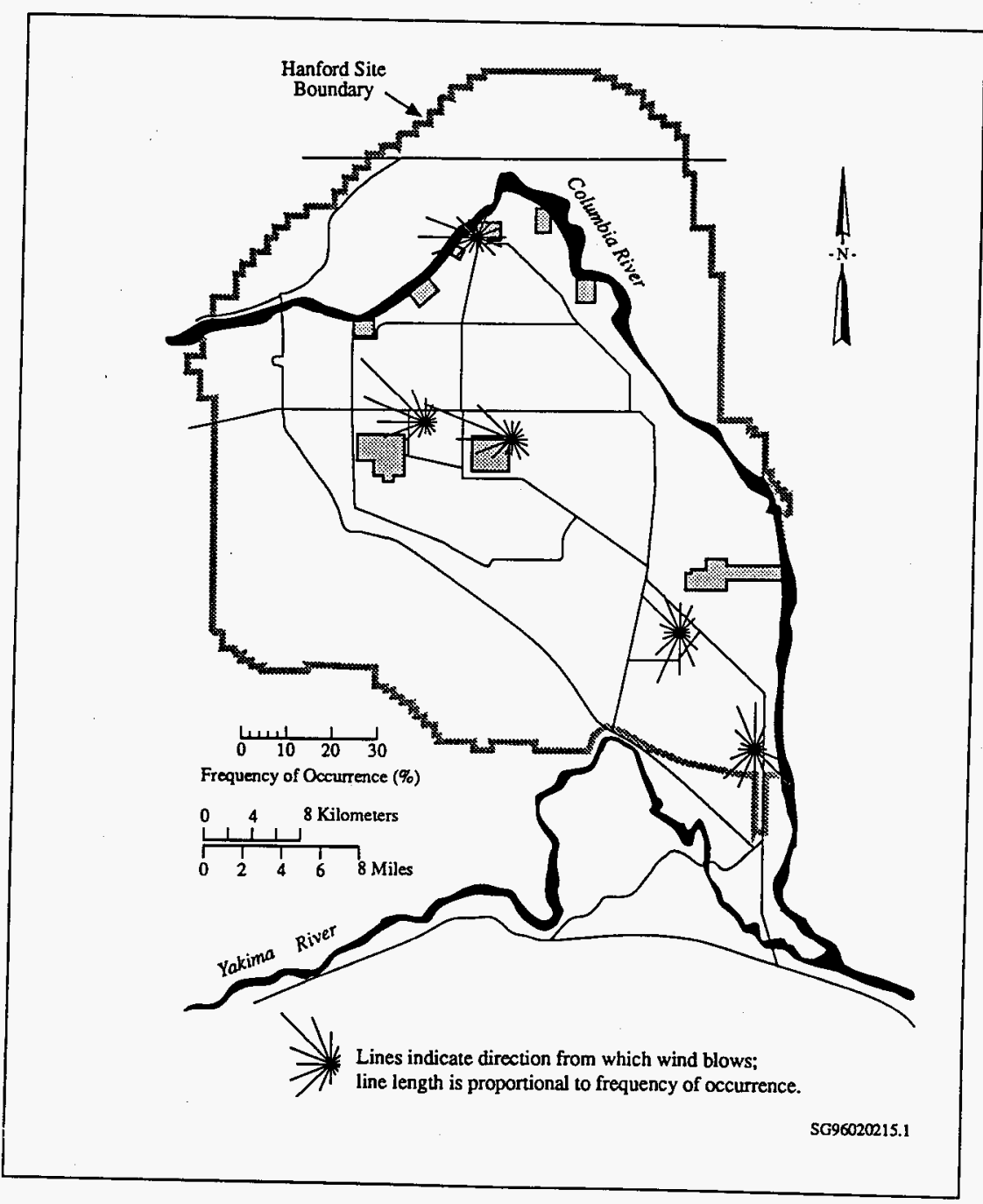


Figure A-2. 100 Area Wind Rose \& Histogram.
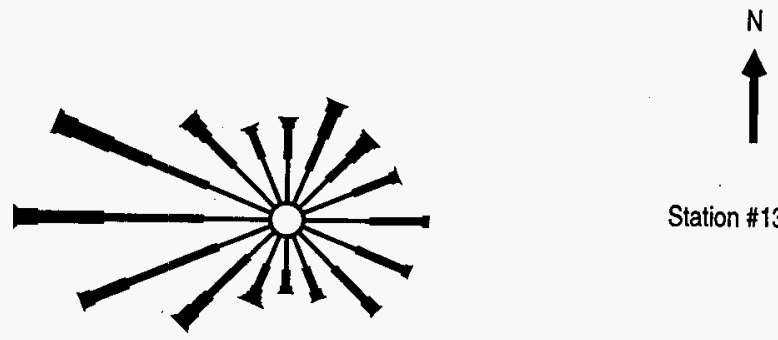

Station \# 13 - 100N

(a) Wind Rose

Period: $1 / 95-12 / 95$

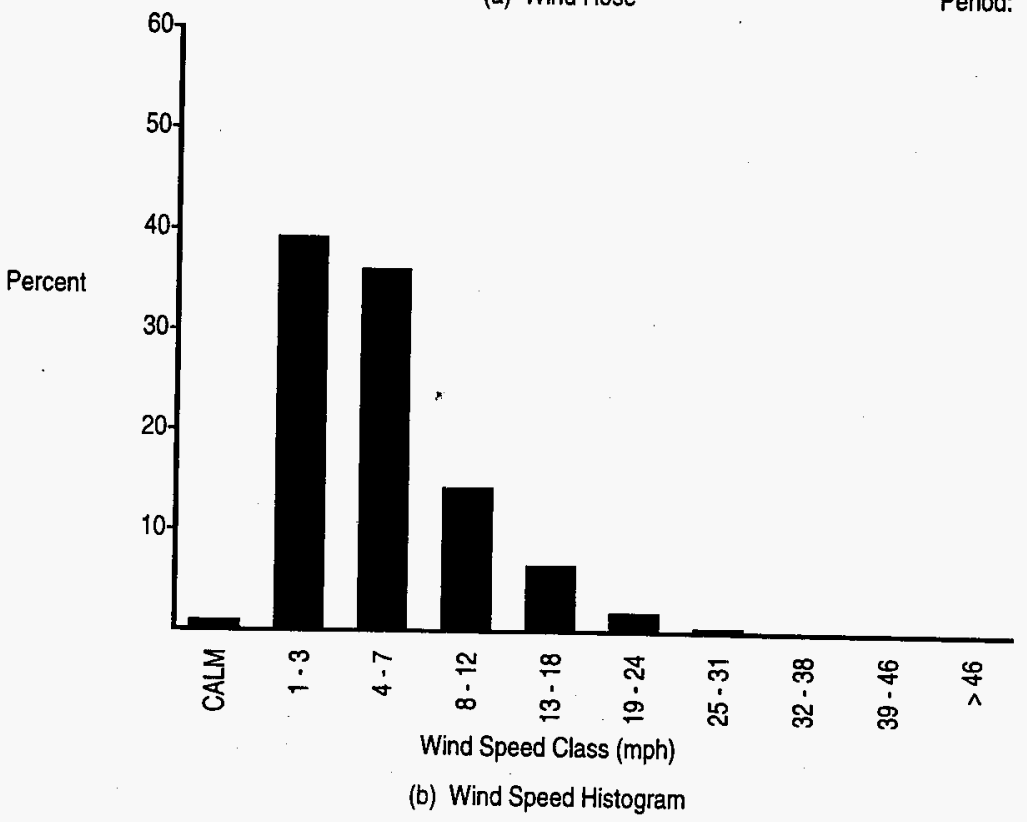

A-4 
Figure A-3. 200 East Area Wind Rose \& Histogram.
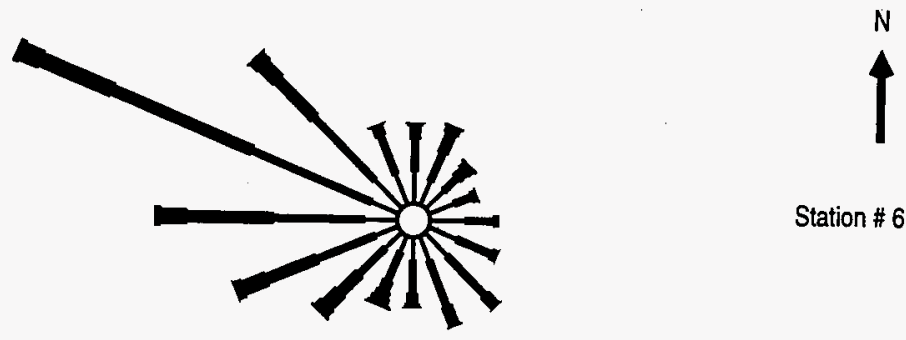

Station \# 6 - 200E

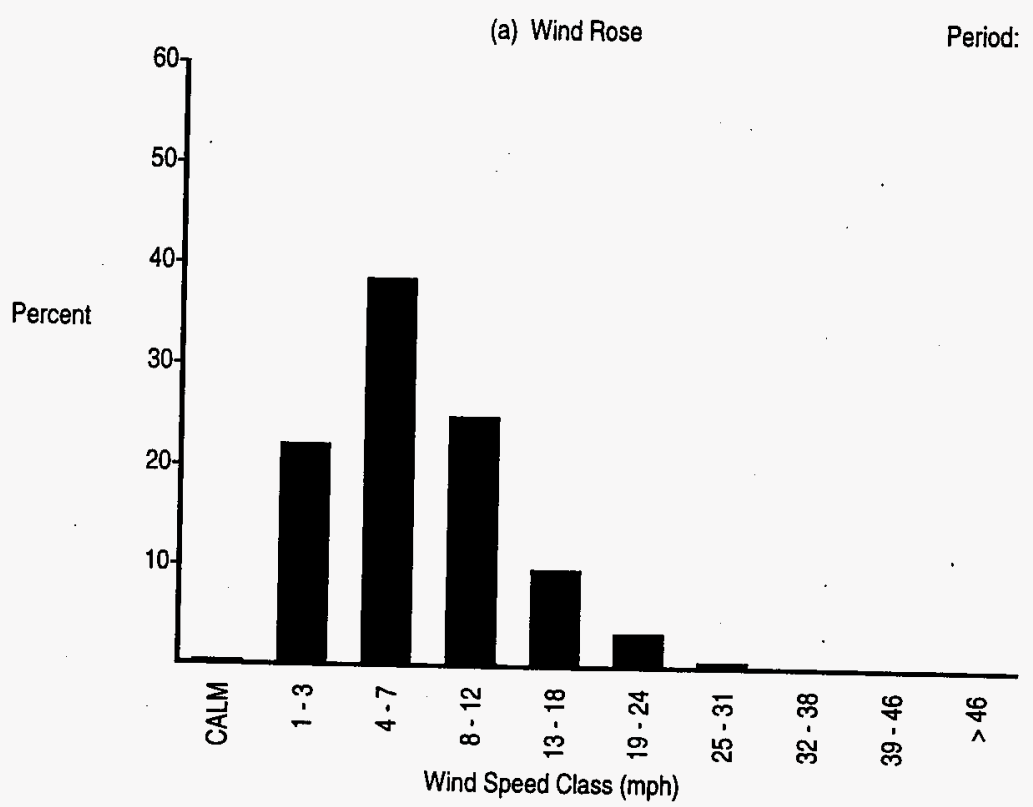

(b) Wind Speed Histogram 
Figure A-4. 200 West Area Wind Rose \& Histogram.
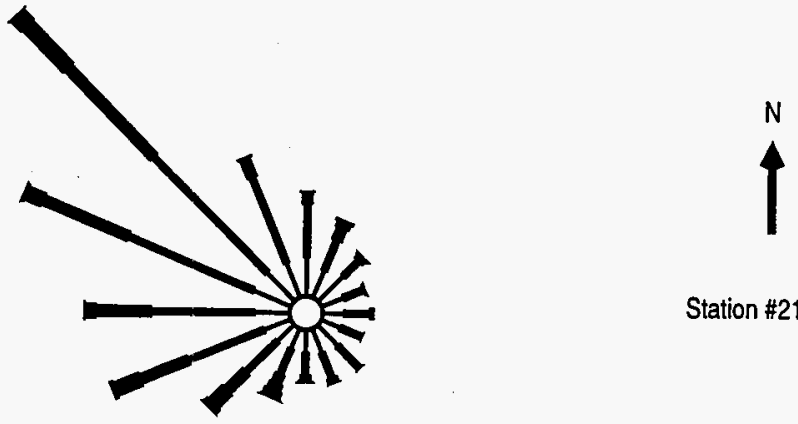

Station \#21 - HMS

(a) Wind Rose

Period: $1 / 95-12 / 95$

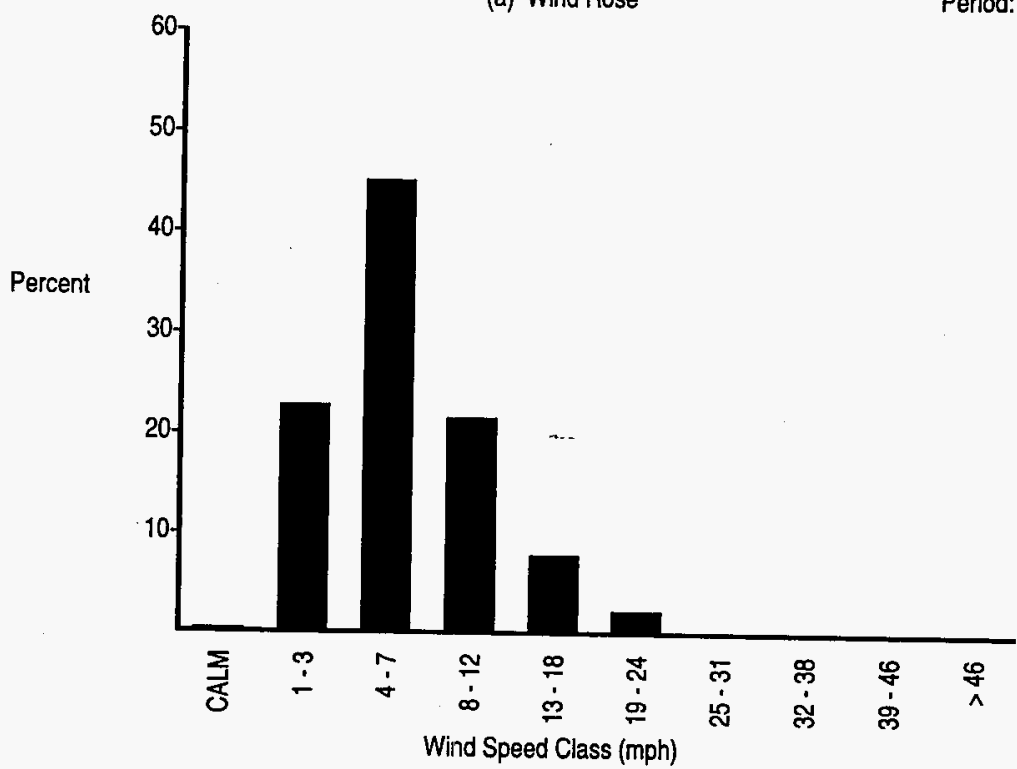

(b) Wind Speed Histogram 
Figure A-5. 300 Area Wind Rose \& Histogram.
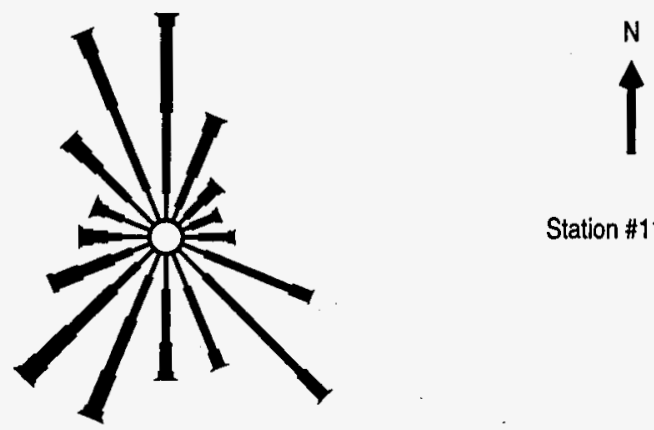

Station \#11 - 300A

(a) Wind Rose

Period: $1 / 95 \cdot 12 / 95$

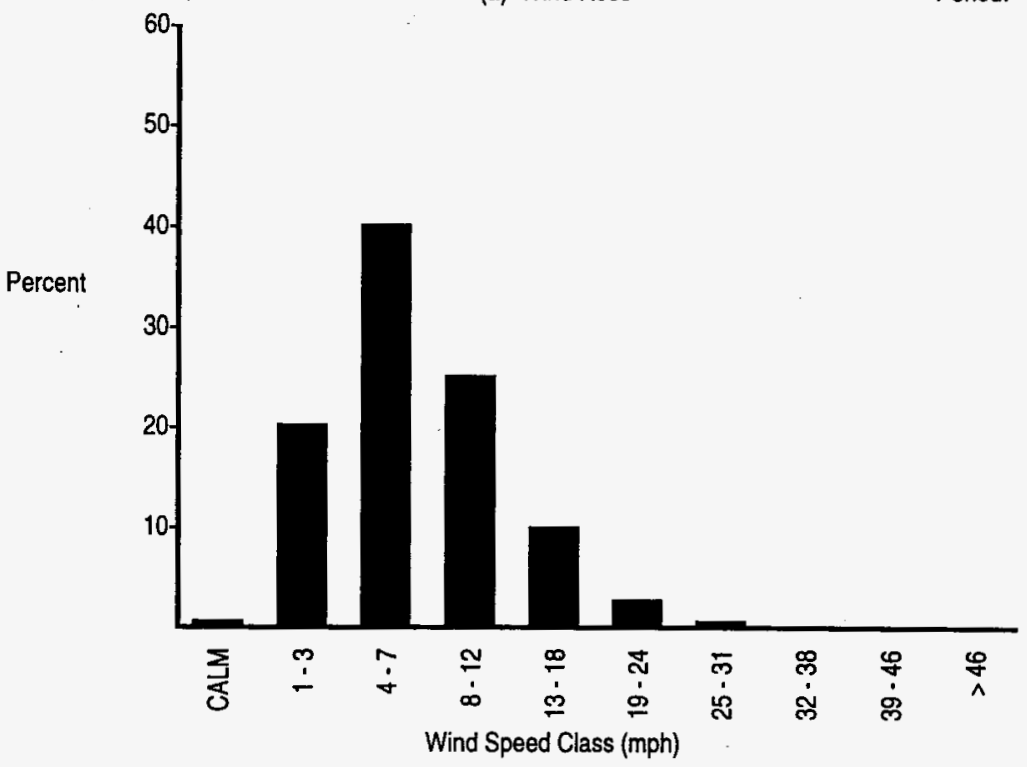

(b) Wind Speed Histogram 
Figure A-6. 400 Area Wind Rose \& Histogram.
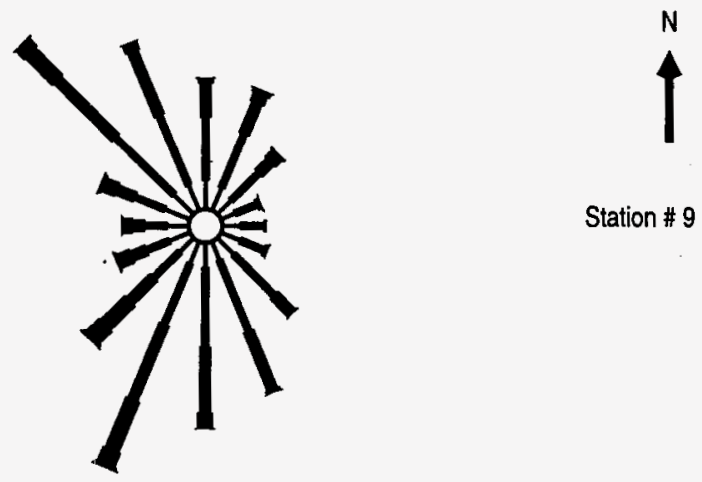

Station \# 9 - FFTF

(a) Wind Rose

Period: 1/95 - 12/95

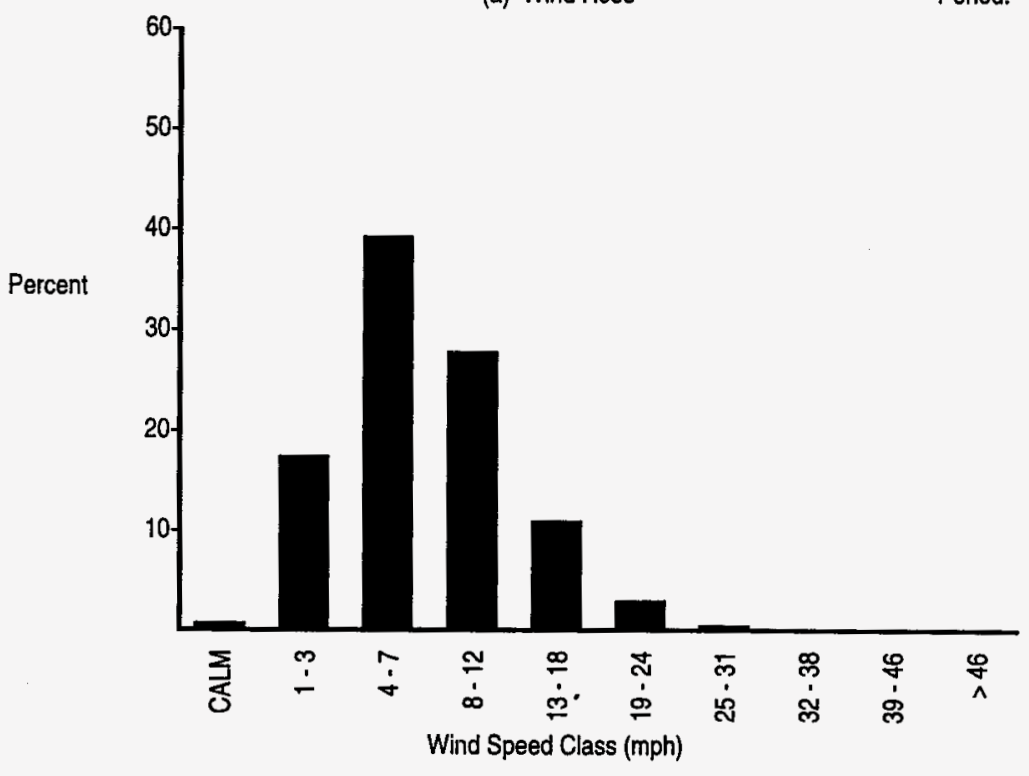

(b) Wind Speed Histogram 
Table A-1. Annual Average Dispersion Factor Around the 100 Area During 1995 for a 10-Meter Release Height.

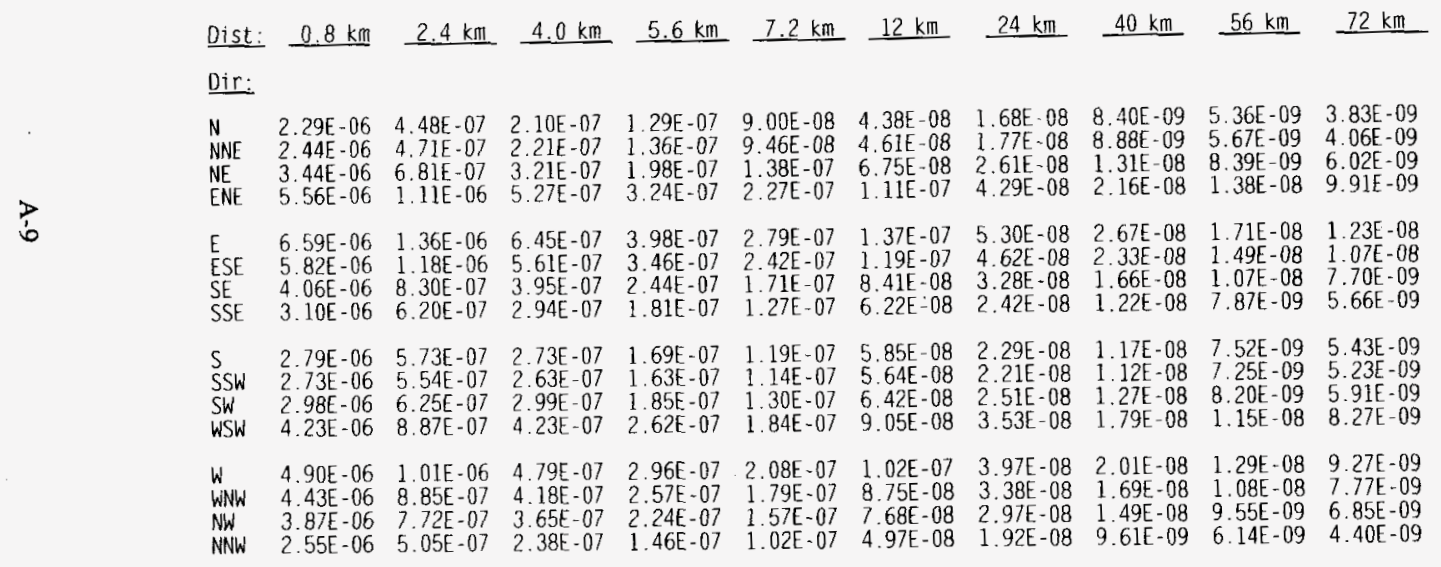


Table A-2. Annual Average Dispersion Factor Around the 200 Areas During 1995 for an 89-Meter Release Height.

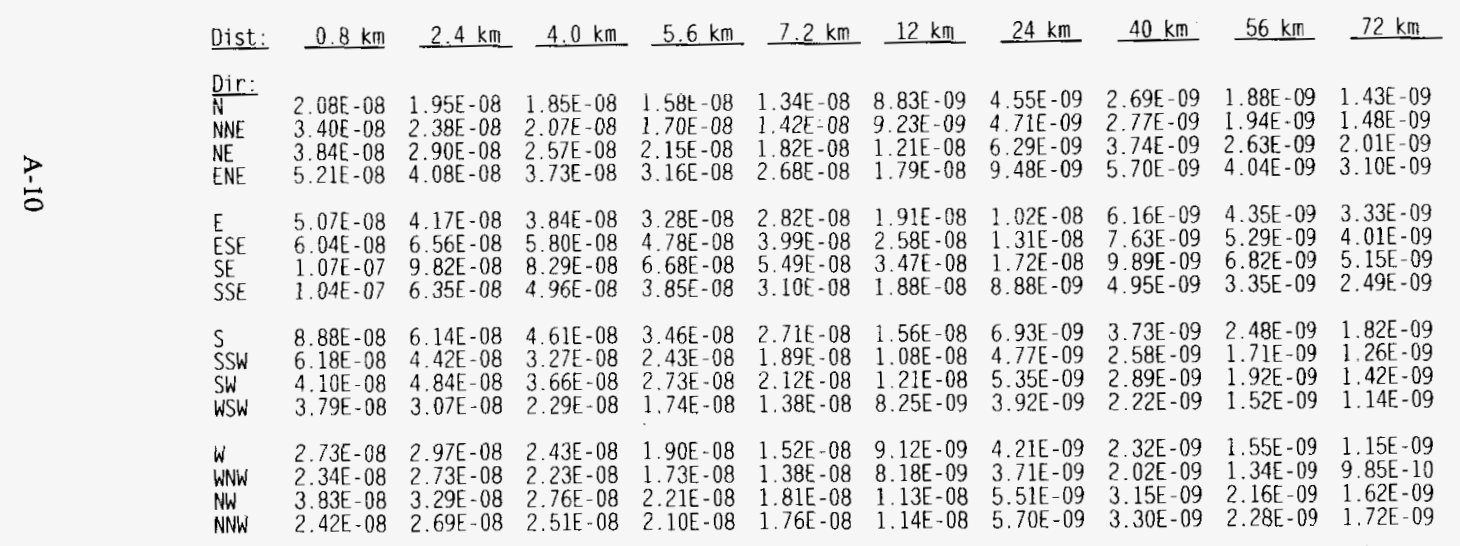


Table A-3. Annual Average Dispersion Factor Around the 300 Area During 1995 for a 10-Meter Release Height.

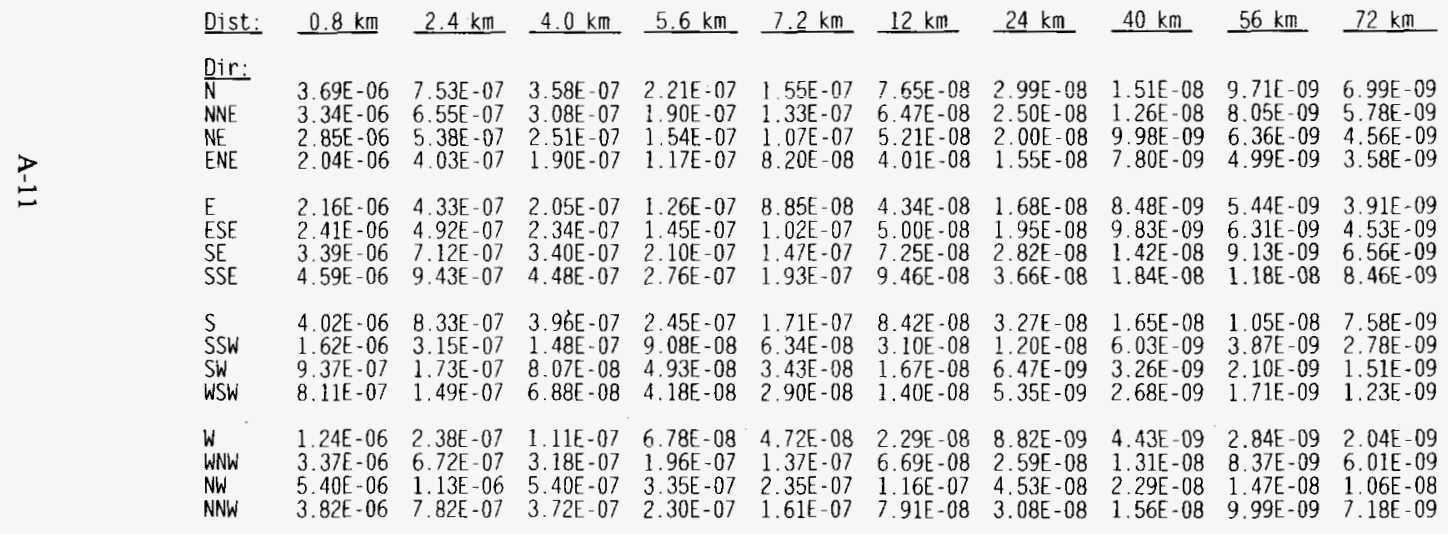


Table A-4. Annual Average Dispersion Factor Around the 400 Area During 1995 for a 10-Meter Release Height.

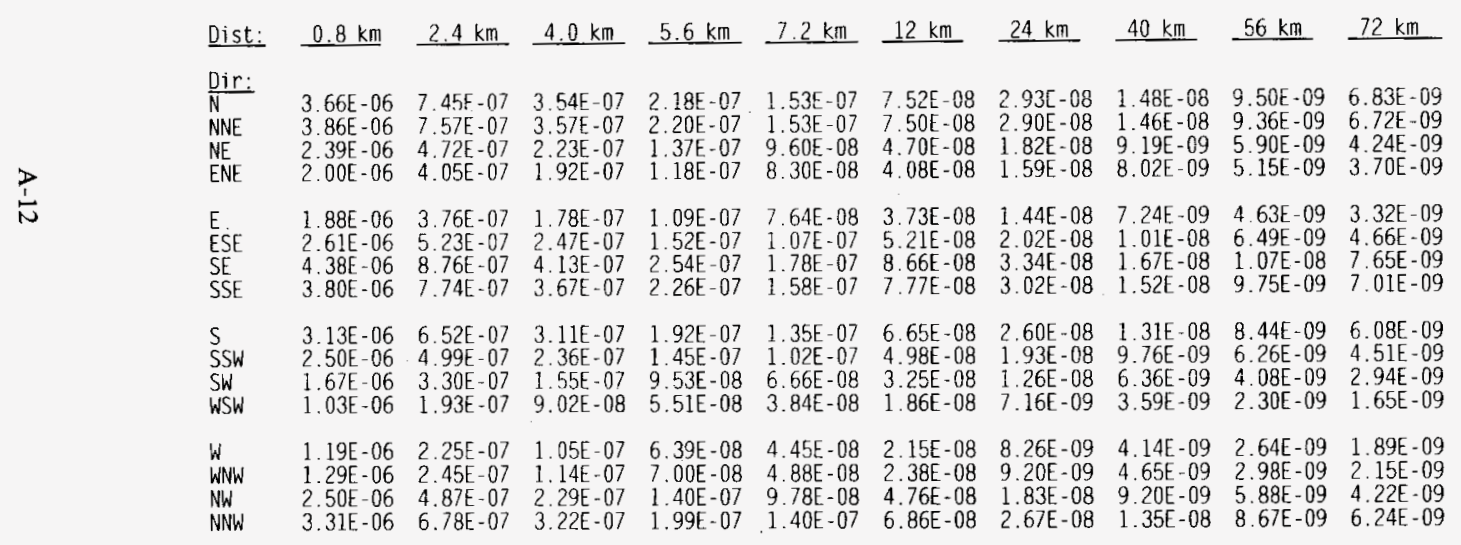


DOE/RL-96-37

Table A-5. Radionculide Data Used for CAP88-PC Dose Calculations at Hanford.

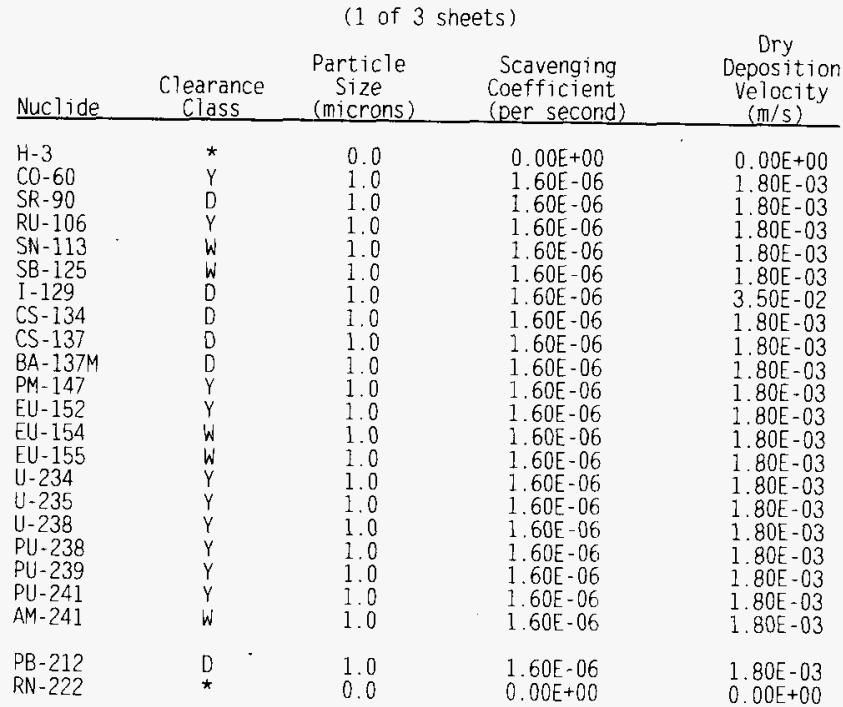


DOE/RL-96-37

Table A-5. Radionculide Data Used for CAP88-PC Dose Calculations at Hanford.

( 2 of 3 sheets)

\begin{tabular}{|c|c|c|c|c|c|}
\hline \multirow[b]{2}{*}{ Nuclide } & \multicolumn{3}{|c|}{ DECAY CONSTANT (PER DAY) } & \multicolumn{2}{|c|}{ IRANSFER COEFFICIENT } \\
\hline & $\begin{array}{l}\text { Radio- } \\
\text { active (1) }\end{array}$ & Surface & Water & $M i l k$ (2) & Meat (3) \\
\hline 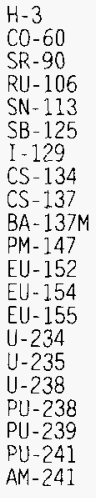 & $\begin{array}{l}0.00 \mathrm{E}+00 \\
0.00 \mathrm{E}+00 \\
0.00 \mathrm{E}+00 \\
0.00 \mathrm{E}+00 \\
0.00 \mathrm{E}+00 \\
0.00 \mathrm{E}+00 \\
0.00 \mathrm{E}+00 \\
0.00 \mathrm{E}+00 \\
0.00 \mathrm{E}+00 \\
3.91 \mathrm{E}+02 \\
0.00 \mathrm{E}+00 \\
0.00 \mathrm{E}+00 \\
0.00 \mathrm{E}+00 \\
0.00 \mathrm{E}+00 \\
0.00 \mathrm{E}+00 \\
0.00 \mathrm{E}+00 \\
0.00 \mathrm{E}+00 \\
0.00 \mathrm{E}+00 \\
0.00 \mathrm{E}+00 \\
0.00 \mathrm{E}+00 \\
0.00 \mathrm{E}+00\end{array}$ & $\begin{array}{l}5.48 \mathrm{E}-05 \\
5.48 \mathrm{E}-05 \\
5.48 \mathrm{E}-05 \\
5.48 \mathrm{E}-05 \\
5.48 \mathrm{E}-05 \\
5.48 \mathrm{E}-05 \\
5.48 \mathrm{E}-05 \\
5.48 \mathrm{E}-05 \\
5.48 \mathrm{E}-05 \\
5.48 \mathrm{E}-05 \\
5.48 \mathrm{E}-05 \\
5.48 \mathrm{E}-05 \\
5.48 \mathrm{E}-05 \\
5.48 \mathrm{E}-05 \\
5.48 \mathrm{E}-05 \\
5.48 \mathrm{E}-05 \\
5.48 \mathrm{E}-05 \\
5.48 \mathrm{E}-05 \\
5.48 \mathrm{E}-05 \\
5.48 \mathrm{E}-05 \\
5.48 \mathrm{E}-05\end{array}$ & $\begin{array}{l}0.00 \mathrm{E}+00 \\
0.00 \mathrm{E}+00 \\
0.00 \mathrm{E}+00 \\
0.00 \mathrm{E}+00 \\
0.00 \mathrm{E}+00 \\
0.00 \mathrm{E}+00 \\
0.00 \mathrm{E}+00 \\
0.00 \mathrm{E}+00 \\
0.00 \mathrm{E}+00 \\
0.00 \mathrm{E}+00 \\
0.00 \mathrm{E}+00 \\
0.00 \mathrm{E}+00 \\
0.00 \mathrm{E}+00 \\
0.00 \mathrm{E}+00 \\
0.00 \mathrm{E}+00 \\
0.00 \mathrm{E}+00 \\
0.00 \mathrm{E}+00 \\
0.00 \mathrm{E}+00 \\
0.00 \mathrm{E}+00 \\
0.00 \mathrm{E}+00 \\
0.00 \mathrm{E}+00\end{array}$ & $\begin{array}{l}0.00 \mathrm{E}+00 \\
2.00 \mathrm{E}-03 \\
1.50 \mathrm{E}-03 \\
6.00 \mathrm{E}-07 \\
1.00 \mathrm{E}-03 \\
1.00 \mathrm{E}-04 \\
1.00 \mathrm{E}-02 \\
7.00 \mathrm{E}-03 \\
7.00 \mathrm{E}-03 \\
3.50 \mathrm{E}-04 \\
2.00 \mathrm{E}-05 \\
2.00 \mathrm{E}-05 \\
2.00 \mathrm{E}-05 \\
2.00 \mathrm{E}-05 \\
6.00 \mathrm{E}-04 \\
6.00 \mathrm{E}-04 \\
6.00 \mathrm{E}-04 \\
1.00 \mathrm{E}-07 \\
1.00 \mathrm{E}-07 \\
1.00 \mathrm{E}-07 \\
4.00 \mathrm{E}-07\end{array}$ & $\begin{array}{l}0.00 \mathrm{E}+00 \\
2.00 \mathrm{E}-02 \\
3.00 \mathrm{E}-04 \\
2.00 \mathrm{E}-03 \\
8.00 \mathrm{E}-02 \\
1.00 \mathrm{E}-03 \\
7.00 \mathrm{E}-03 \\
2.00 \mathrm{E}-02 \\
2.00 \mathrm{E}-02 \\
1.50 \mathrm{E}-04 \\
5.00 \mathrm{E}-03 \\
5.00 \mathrm{E}-03 \\
5.00 \mathrm{E}-03 \\
5.00 \mathrm{E}-03 \\
2.00 \mathrm{E}-04 \\
2.00 \mathrm{E}-04 \\
2.00 \mathrm{E}-04 \\
5.00 \mathrm{E}-07 \\
5.00 \mathrm{E}-07 \\
5.00 \mathrm{E}-07 \\
3.50 \mathrm{E}-06\end{array}$ \\
\hline $\begin{array}{l}\text { PB-212 } \\
\text { RN }-222\end{array}$ & $\begin{array}{l}1.56 \mathrm{E}+00 \\
1.81 \mathrm{E}-01\end{array}$ & $\begin{array}{l}5.48 \mathrm{E}-05 \\
5.48 \mathrm{E}-05\end{array}$ & $\begin{array}{l}0.00 E+00 \\
0.00 E+00\end{array}$ & $\begin{array}{l}2.50 E-04 \\
0.00 E+00\end{array}$ & $\begin{array}{l}3.00 \mathrm{E}-04 \\
0.00 \mathrm{E}+00\end{array}$ \\
\hline
\end{tabular}

FOOTNOTES: (1) Effective radioactive decay constant in plume: set to zero if less than $1.0 E-2$

(2) Fraction of animal's dajly intake of nuclide which appears in each $L$ of milk (days $/ L$ )

(3) Fraction of animal's daily intake of nuclide which appears in each $\mathrm{kg}$ of meat (days $/ \mathrm{kg}$ ) 
DOE/RL-96-37

Table A-5. Radionculide Data Used for CAP88-PC Dose Calculations at Hanford.

( 3 of 3 sheets)

\begin{tabular}{|c|c|c|c|c|}
\hline \multirow[b]{2}{*}{ Nuclide } & \multicolumn{2}{|c|}{$\begin{array}{l}\text { CONCENTRATION } \\
\text { UPTAKE FACTOR }\end{array}$} & \multicolumn{2}{|c|}{ GI UPTAKE FRACTION } \\
\hline & Forage (1) & Edible (2) & Inhalation & Ingestion \\
\hline $\begin{array}{l}H-3 \\
C O-60 \\
\text { SR-90 } \\
R U-106 \\
I-129 \\
\text { SN-113 } \\
\text { SB-125 } \\
\text { CS-134 } \\
C S-137 \\
\text { BA-137M } \\
P M-147 \\
\text { EU-152 } \\
\text { EU-154 } \\
\text { EU-155 } \\
U-234 \\
U-235 \\
U-238 \\
P U-238 \\
P U-239 \\
P U-241 \\
A M-241\end{array}$ & $\begin{array}{l}0.00 \mathrm{E}+00 \\
2.00 \mathrm{E}-02 \\
2.50 \mathrm{E}+00 \\
7.50 \mathrm{E}-02 \\
1.50 \mathrm{E}-01 \\
3.00 \mathrm{E}-02 \\
2.00 \mathrm{E}-01 \\
8.00 \mathrm{E}-02 \\
8.00 \mathrm{E}-02 \\
1.50 \mathrm{E}-01 \\
1.00 \mathrm{E}-02 \\
1.00 \mathrm{E}-02 \\
1.00 \mathrm{E}-02 \\
1.00 \mathrm{E}-02 \\
8.50 \mathrm{E}-03 \\
8.50 \mathrm{E}-03 \\
8.50 \mathrm{E}-03 \\
4.50 \mathrm{E}-04 \\
4.50 \mathrm{E}-04 \\
4.50 \mathrm{E}-04 \\
5.50 \mathrm{E}-03\end{array}$ & $\begin{array}{l}0.00 \mathrm{E}+00 \\
3.00 \mathrm{E}-03 \\
1.07 \mathrm{E}-01 \\
8.56 \mathrm{E}-03 \\
2.14 \mathrm{E}-02 \\
2.57 \mathrm{E}-03 \\
1.28 \mathrm{E}-02 \\
1.28 \mathrm{E}-02 \\
1.28 \mathrm{E}-02 \\
6.42 \mathrm{E}-03 \\
1.71 \mathrm{E}-03 \\
1.71 \mathrm{E}-03 \\
1.71 \mathrm{E}-03 \\
1.71 \mathrm{E}-03 \\
1.71 \mathrm{E}-03 \\
1.71 \mathrm{E}-03 \\
1.71 \mathrm{E}-03 \\
1.93 \mathrm{E}-05 \\
1.93 \mathrm{E}-05 \\
1.93 \mathrm{E}-05 \\
1.07 \mathrm{E}-04\end{array}$ & $\begin{array}{l}9.50 \mathrm{E}-01 \\
5.00 \mathrm{E}-02 \\
3.00 \mathrm{E}-01 \\
5.00 \mathrm{E}-02 \\
9.50 \mathrm{E}-01 \\
2.00 \mathrm{E}-02 \\
1.00 \mathrm{E}-02 \\
9.50 \mathrm{E}-01 \\
9.50 \mathrm{E}-01 \\
1.00 \mathrm{E}-01 \\
3.00 \mathrm{E}-04 \\
1.00 \mathrm{E}-03 \\
1.00 \mathrm{E}-03 \\
1.00 \mathrm{E}-03 \\
2.00 \mathrm{E}-03 \\
2.00 \mathrm{E}-03 \\
2.00 \mathrm{E}-03 \\
1.00 \mathrm{E}-03 \\
1.00 \mathrm{E}-04 \\
1.00 \mathrm{E}-03 \\
1.00 \mathrm{E}-03\end{array}$ & $\begin{array}{l}9.50 \mathrm{E}-01 \\
3.00 \mathrm{E}-01 \\
3.00 \mathrm{E}-01 \\
5.00 \mathrm{E}-02 \\
9.50 \mathrm{E}-01 \\
2.00 \mathrm{E}-02 \\
1.00 \mathrm{E}-01 \\
9.50 \mathrm{E}-01 \\
9.50 \mathrm{E}-01 \\
1.00 \mathrm{E}-01 \\
3.00 \mathrm{E}-04 \\
1.00 \mathrm{E}-03 \\
1.00 \mathrm{E}-03 \\
1.00 \mathrm{E}-03 \\
2.00 \mathrm{E}-01 \\
2.00 \mathrm{E}-01 \\
2.00 \mathrm{E}-01 \\
1.00 \mathrm{E}-03 \\
1.00 \mathrm{E}-03 \\
1.00 \mathrm{E}-03 \\
1.00 \mathrm{E}-03\end{array}$ \\
\hline $\begin{array}{l}P B-212 \\
R N-222\end{array}$ & $\begin{array}{l}4.50 E-02 \\
0.00 E+00\end{array}$ & $\begin{array}{l}3.85 \mathrm{E}-03 \\
0.00 \mathrm{E}+00\end{array}$ & $\begin{array}{l}2.00 E-01 \\
0.00 E+00\end{array}$ & $\begin{array}{l}\text { 2. } .00 \mathrm{E}-01 \\
0.00 \mathrm{E}+00\end{array}$ \\
\hline FOOTNOTES: & \multicolumn{4}{|c|}{$\begin{array}{l}\text { (1) Concentration factor for uptake of nuclide from soil for } \\
\text { pasture and forage (in } \mathrm{pCi} / \mathrm{kg} \text { dry weight per } \mathrm{pCi} / \mathrm{kg} \text { dry } \\
\text { soil) } \\
\text { (2) Concentration factor for uptake of nuclide from soil by } \\
\text { edibie parts of crops ( } \mathrm{n} \mathrm{pCi} / \mathrm{kg} \text { wet weight } \mathrm{per} \mathrm{pCi} / \mathrm{kg} \text { dry } \\
\text { soil) }\end{array}$} \\
\hline
\end{tabular}




\section{DOE/RL-96-37}

Table A-6. Exposure and Consumption Data for the Hanford Site.

( 1 of 2 sheets)

FOOD SOURCE FOR THE MAXIMALLY EXPOSED INDIVIDUAL:

$\begin{array}{rccc} & \text { Local } & \text { Regional } & \text { Imported } \\ \text { Vegetable: } & 1.000 & 0.000 & 0.000 \\ \text { Meat: } & 1.000 & 0.000 & 0.000 \\ \text { Mi lk: } & 1.000 & 0.000 & 0.000\end{array}$

VALUES FOR RADIONUCLIDE-INDEPENDENT VARIABLES:

HUMAN INHALATION RATE

Cubic certimeters/hr $\quad 9.70 E+05$

SOIL PARAMETERS

Effective surface density ( $\mathrm{kg} / \mathrm{sq} \mathrm{m}$. dry weight)

(Assumes $15 \mathrm{~cm}$ plow layer)

$2.24 E+02$

BUILOUP TIMES

For activity in soil (years)

For radionuclides deposited on ground/water (days) $\quad 1.83 \mathrm{E}+04$

DELAY TIMES

Ingestion of pasture grass by animals (hr)

$0.00 E+00$

Ingestion of stored feed by animals (hr)

2. $40 E+03$

Ingestion of leafy vegetables by man (hr)

2. $40 E+01$

Ingestion of produce by man (hr)

Transport time from animal feed-milk-man (day)

1. $20 \mathrm{E}+02$

$2.00 E+00$

Time from slaughter to consumption (day)

$1.50 \mathrm{E}+01$

WEATHERING

Removal rate constant for physical loss (per hr)

3.00E-03

CROP EXPOSURE DURATION

Pasture grass (hr)

Crops/leafy vegetables ( $h r$ )

7. $20 \mathrm{E}+02$

2. $16 \mathrm{E}+03$

AGRICULTURAL PRODUCTIVITY

Gras5-cow-mi $7 k$-man pathway ( $\mathrm{kg} / \mathrm{sq} \mathrm{m})$

Produce/leafy veg for human consumption ( $\mathrm{kg} / \mathrm{sq} \mathrm{m})$

1. $50 \mathrm{E}+00$

$2.00 \mathrm{E}+00$ 


\section{DOE/RL-96-37}

Table A-6. Exposure and Consumption Data for the Hanford Site.

(2 of 2 sheets)

\begin{tabular}{|c|c|}
\hline $\begin{array}{l}\text { Vegetables } \\
\text { Pasture }\end{array}$ & $\begin{array}{l}2.50 \mathrm{E}-01 \\
2.50 \mathrm{E}-01\end{array}$ \\
\hline $\begin{array}{l}\text { GRAZING PARAMETERS } \\
\text { Fraction of year animals graze on pasture } \\
\text { Fraction of daily feed that is pasture grass } \\
\text { when animal grazes on pasture }\end{array}$ & $\begin{array}{l}7.50 E-01 \\
1.00 E+00\end{array}$ \\
\hline $\begin{array}{l}\text { ANIMAL FEEO CONSUMPTION FACTORS } \\
\text { Contaminated feed/forage ( } \mathrm{kg} / \text { day, dry weight) }\end{array}$ & 1. $.56 \mathrm{E}+01$ \\
\hline $\begin{array}{l}\text { DAIRY PRODUCTIVITY } \\
\text { Milk production of COW }(\mathrm{L} / \text { day })\end{array}$ & 1. $10 \mathrm{E}+01$ \\
\hline $\begin{array}{l}\text { MEAT ANTMAL SLAUGHTER PARAMETERS } \\
\text { MuScle masS of animal at slaughter ( } \mathrm{kg}) \\
\text { Fraction of herd slaughtered (per day) }\end{array}$ & $\begin{array}{l}2.00 \mathrm{E}+02 \\
3.81 \mathrm{E}-03\end{array}$ \\
\hline $\begin{array}{l}\text { DECONTAMINATION } \\
\text { Fraction of radioactivity retained after washing } \\
\text { for leafy vegetables and produce }\end{array}$ & 1. $00 E+00$ \\
\hline $\begin{array}{l}\text { FRACTIONS GROWN IN GARDEN OF INTEREST } \\
\text { Produce ingested } \\
\text { Leafy vegetables ingested }\end{array}$ & $\begin{array}{l}1.00 E+00 \\
1.00 E+00\end{array}$ \\
\hline $\begin{array}{l}\text { INGESTION RATIOS: } \\
\text { IMMEOIATE SURROLNDING AREA/TOTAL WITHIN AREA } \\
\text { Vegetables } \\
\text { Meat } \\
\text { Milk }\end{array}$ & $\begin{array}{l}\text { 1. } 00 \mathrm{E}+00 \\
\text { 1. } 00 \mathrm{E}+00 \\
\text { 1. } 00 \mathrm{E}+00\end{array}$ \\
\hline $\begin{array}{l}\text { MINIMUM INGESTION FRACTIONS FROM OUTSIDE AREA } \\
\text { (Minimum fractions of food types from outside } \\
\text { area listed below are actual fixed values.) } \\
\text { Vegetabies } \\
\text { Meat } \\
\text { Mi } \backslash k\end{array}$ & $\begin{array}{l}0.00 E+00 \\
0.00 E+00 \\
0.00 E+00\end{array}$ \\
\hline $\begin{array}{l}\text { HUMAN FOOO UTILIZATION FACTORS } \\
\text { Produce ingestion }(\mathrm{kg} / \mathrm{y}) \\
\text { Milk ingestion }(\mathrm{L} / \mathrm{y}) \\
\text { Meat ingestion }(\mathrm{kg} / \mathrm{y}) \\
\text { Leafy vegetable ingestion }(\mathrm{kg} / \mathrm{y})\end{array}$ & $\begin{array}{l}2.20 E+02 \\
2.70 E+02 \\
9.80 E+01 \\
3.00 E+01\end{array}$ \\
\hline $\begin{array}{l}\text { SWIMMING PARAMETERS } \\
\text { Fraction of time spent swimming } \\
\text { Dilution factor for water }(\mathrm{cm})\end{array}$ & $\begin{array}{l}\text { 1. } 00 E-02 \\
1.00 E+00\end{array}$ \\
\hline
\end{tabular}


DOE/RL-96-37

Table A-7. Hanford Site Meteorological Data - General Site Information.

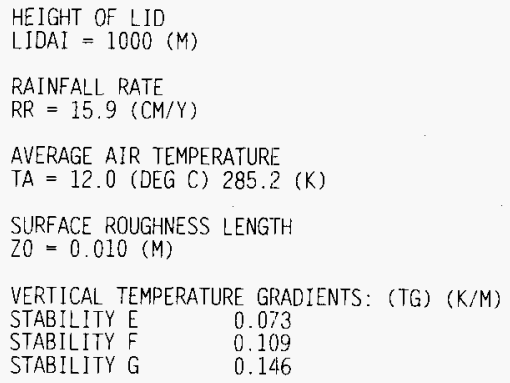




\section{DISTRIBUTION}

Number of Copies

\section{OFFSITE}

G. M. Leitch (5)

A. Frankel

R. W, Poeten

Washington State Department of Health

Air Emissions and Defense Waste Section

Division of Radiation Protection

P. O. Box 47827

Olympia, WA $98504-7827$
A. W. Conklin (5)
J. E. Erickson

1

Washington State Department of Ecology

M. A. Wilson

B5-18

5

U.S. Department of Energy-Headquarters

R. F. Pelletier (3)

EH-232

M. K. Harmon

EM-442

K. C. Duvall

EH-232

Confederated Tribes of the Umatilla Indian Reservation

P. O. Box 638

Pendleton, OR 97801

J. R. Wilkinson 
DOE/RL-96-37

\section{DISTRIBUTION (continued)}

Number of Copies

OFFSITE

1

Nez Perce Tribe

Environmental Restoration and Waste Management

P. O. Box 365

Lapwai, ID 83540

D. L. Powaukee

1

Yakama Indian Nation

Environmental Restoration Waste Management Program

P.O. Box 151

Toppenish, Washington 98948

R. Jim

\section{ONSITE}

G. M. Bell

R. F. Brich

H0-12

J. B. Hall

A5-15

J. E. Mecca

R3-81

B. M. Pangborn

A5-55

J. E. Rasmussen

A5-15

H. M. Rodriguez (5)

D. C. Ward

A. H. Wirkkala

A.5-11

DOE-RL Public Reading Room

H2-53

14

Pacific Northwest National Laboratory

M. Y. Ballinger

P7-68

E. G. Damberg

P7-68

R. L. Dirkes

K6-75

W. T. Farris

K3-54 
DOE/RL-96-37

\section{DISTRIBUTION (continued)}

\section{Number of Copies}

ONSITE

Pacific Northwest National Laboratory (continued)

$\begin{array}{lc}\text { R. W. Hanf, Jr. } & \text { K6-75 } \\ \text { G. R. Hoenes } & \text { P7-79 } \\ \text { S. J. Jette } & \text { P7-68 } \\ \text { T. J. Lazarski } & \text { P7-79 } \\ \text { T. W. Moon } & \text { P7-79 } \\ \text { K. Rhoads } & \text { K3-54 } \\ \text { M. J. Sula } & \text { P7-68 } \\ \text { H. T. Tilden II } & \text { P7-79 } \\ \text { R. K. Woodruff } & \text { P7-68 } \\ \text { PNNL Reference Library } & \text { K1-11 }\end{array}$

9

Bechtel Hanford, Inc.

E. T. Coenenburg H9-11

R. G. Egge T7-05

M. E. Greenidge X5-54

J. T. Hadley H9-11

R. J. Landon H0-18

L. A. Mihalik H9-12

M. R. Morton X5-53

D. W. Long T7-05

$\begin{array}{ll}\text { J. P. Zoric } & \text { X5-57 }\end{array}$

151 Westinghouse Hanford Company

President's Office B3-01

D. J. Alexander S3-24

D. Alison R1-51

D. D. Bachand R1-51

J. M. Barnett T4-08

J. A. Bates H6-36

R. J. Boom T6-12

M. J. Brown S6-71

K. R. Busching T4-03

G. J. Carter, Jr. X5-53

S. E. Campbell T4-03

Distr-3 
DOE/RL-96-37

DISTRIBUTION (continued)

Number of Copies

ONSITE

Westinghouse Hanford Company (continued)

B. C. Cornwell

L5-70

J. F. Brown

S5-03

G. M. Crummel

R1-51

N. R. Dahl

N2-57

W. E. Davis

H6-36

J. L. Demarest

G1-40

L. P. Diediker (75)

H6-36

A. J. Diliberto

H6-10

T. A. Dillhoff

N2-57

B. J. Dixon

B4-20

W. T. Dixon

H6-21

J. J. Dorian

H6-30

D. L. Dyekman

T4-08

D. L. Edwards

S3-27

B. G. Erlandson

R2-36

D. J. Farquhar

H7-34

D. P. Fasset

T3-28

D. L. Flyckt

S6-71

T. P. Frazier

H6-25

R. G. Gant

X3-79

L. A. Garner

R2-36

C. K. Girres

T3-28

B. P. Gleckler

H6-36

F. T. Green

H6-29

P. R. Gunter

R1-52

R. D. Gustavson

R2-50

K. J. Hlaggerty

L6-04

D. L. Halgren

L6-04

M. J. Hall

T6-12

M. B. Jaeger

L4-02

A. R. Johnson

H6-20

D. L. Johnson

S5-66

R. E. Johnson

H6-25

S. E. Killoy

S4-66

J. M. Kisielnicki

L6-39 
DOE/RL-96-37

DISTRIBUTION (continued)

Number of Copies

ONSITE

Westinghouse Hanford Company (continued)

G. J. LeBaron S6-19

L. L. Lockrum S3-90

J. J. Luke H6-25

B. M. Markes H6-20

C. E. Marple $\$ 4-56$

B. A. Mayancsik T4-03

D. J. McBride T5-54

P. C. Miller R1-51

D. L. Mitchell H6-29

J. A. Morrison S3-31

S. E. Myers S3-24

S. T. Noga $\quad$ S5-66

J. K. Perry H6-25

A. D. Poor L6-55

D. E. Rasmussen $\quad \mathrm{N} 1-47$

J. H. Rasmussen S6-51

T. M. Ridge S4-66

J. W. Schmidt (2) H6-20

P. J. Sullivan T5-54

R. W. Szelmeczka L6-05

L. W. Vance B1-13

E. F. Votaw G1-13

D. J. Watson X3-79

L. L. Weaver T6-12

C. D. Wollam \$6-22

M. T. York H6-29

Central Files A3-88

Correspondence Control A3-01

DPC A3-89 
THSPAGE INTENTONALI

LEFT BLAR 